\title{
Efficient Integrated Circuits for Wideband Wireless Transceivers
}

\author{
Duong Quoc Tai
}

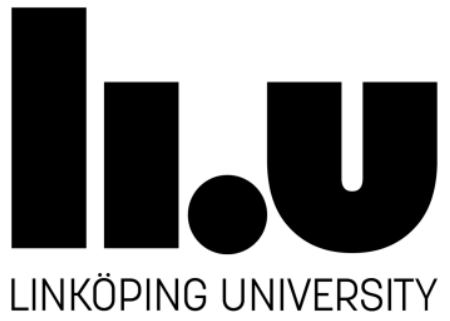

Department of Electrical Engineering

Linköping University, SE-581 83 Linköping, Sweden

Linköping 2016

ISBN 978-91-7685-904-9

ISSN 0345-7524 


\section{Efficient Integrated Circuits for Wideband Wireless Transceivers}

Duong Quoc Tai

Copyright (C) Duong Quoc Tai, 2016

ISBN 978-91-7685-904-9

Linköping Studies in Science and Technology

Dissertations, No. 1722

ISSN: 0345-7524

Division of Integrated Circuits and Systems

Department of Electrical Engineering (ISY)

Institute of Technology

Linköping University

SE-581 83 Linköping

Sweden

\section{Cover image:}

The cover image illustrates a wireless transceiver where LNTA and DAC are emphasized. The back image is the symbol of wireless radio frequency (RF).

Printed by LiU-Tryck, Linköping University

Linköping, Sweden, 2016 


\section{Abstract}

The proliferation of portable communication devices combined with the relentless demand for higher data rates has spurred the development of wireless communication standards which can support wide signal bandwidths. Benefits of the complementary metal oxide semiconductor (CMOS) process such as high device speeds and low manufacturing cost have rendered it the technology of choice for implementing wideband wireless transceiver integrated circuits (ICs). This dissertation addresses the key challenges encountered in the design of wideband wireless transceiver ICs. It is divided into two parts. Part I describes the design of crucial circuit blocks such as a highly selective wideband radio frequency (RF) front-end and an on-chip test module which are typically found in wireless receivers. The design of high-speed, capacitive DACs for wireless transmitters is included in Part II.

The first work in Part $\mathbf{I}$ is the design and implementation of a wideband RF frontend in 65-nm CMOS. To achieve blocker rejection comparable to surface-acousticwave (SAW) filters, the highly selective and tunable RF receiver utilizes impedance transformation filtering along with a two-stage architecture. It is well known that the low-noise amplifier (LNA) which forms the first front-end stage largely decides the receiver performance in terms of noise figure (NF) and linearity (IIP3/P1dB). The proposed LNA uses double cross-coupling technique to reduce NF while complementary derivative superposition (DS) and resistive feedback are employed to achieve high linearity. The resistive feedback also enhances input matching. In measurements, the front-end achieves performance comparable to SAW filters with blocker rejection greater than $38 \mathrm{~dB}$, NF 3.2-5.2 dB, out-of-band IIP3 > +17 dBm and blocker $\mathrm{P} 1 \mathrm{~dB}>+5 \mathrm{dBm}$ over a frequency range of $0.5-3 \mathrm{GHz}$.

The second work in Part $\mathbf{I}$ is the design of an RF amplitude detector for on-chip test. As the complexity of RF ICs continues to grow, the task of testing and debugging 
them becomes increasingly challenging. The degradation in performance or the drift from the optimal operation points may cause systems to fail. To prevent this effect and ensure acceptable performance in the presence of process, voltage and temperature variations (PVT), test and calibration of the RF ICs become indispensable. A wideband, high dynamic range RF amplitude detector design aimed at on-chip test is proposed. Gain-boosting and sub-ranging techniques are applied to the detection circuit to increase the gain over the full range of input amplitudes without compromising the input impedance. A technique suitable for on-chip third/second-order intercept point (IP3/IP2) test by embedded RF detectors is also introduced.

Part II comprises the design and analysis of high-speed switched-capacitor (SC) DACs for $60-\mathrm{GHz}$ radio transmitters. The digital-to-analog converter (DAC) is one of the fundamental building blocks of transmitters. SC DACs offer several advantages over the current-steering DAC architecture. Specifically, lower capacitor mismatch helps the SC DAC to achieve higher linearity. The switches in the SC DAC are realized by MOS transistors in the triode region which substantially relaxes the voltage headroom requirement. Consequently, SC DACs can be implemented using lower supply voltages in advanced CMOS process nodes compared to their current-steering counterparts. The first work in Part II analyzes the factors limiting the performance of capacitive pipeline DACs. It is shown that the DAC performance is limited mainly by the clock feed-through and settling effects in the SC arrays while the impact of capacitor mismatch and $\mathrm{kT} / \mathrm{C}$ noise are found to be negligible. Based on this analysis, the second work in Part II proposes the split-segmented SC array DAC to overcome the clock feed-through problem since this topology eliminates pipelined charge propagation. Implemented in 65-nm CMOS, the 12-bit SC DAC achieves a Spurious Free Dynamic Range (SFDR) greater than $44 \mathrm{~dB}$ within the input signal bandwidth (BW) of $1 \mathrm{GHz}$ with on-chip memory embedded for digital data generation. Power dissipation is $50 \mathrm{~mW}$ from $1.2 \mathrm{~V}$ supply. Similar performance is achieved with a lower supply voltage $(0.9 \mathrm{~V})$ which shows the scalability of the SC DAC for more advanced CMOS technologies. Furthermore, the proposed SC DAC satisfies the spectral mask of the IEEE 802.11ad WiGig standard with a second-order reconstruction filter and hence it can be used for the $60-\mathrm{GHz}$ radio baseband. 


\section{Sammanfattning}

Spridningen av portabla kommunikationsenheter kombinerat med de ständigt ökade kraven på högre datatakter har drivit på utvecklingen av trådlösa kommunikationsstandarder som stödjer bredbandiga signaler. Fördelar med komplementär metaloxidshalvledare (CMOS) processer så som hög komponenthastighet samt låg tillverkningskostnad har gjort det till den dominerade tekniken för att implementera bredbandiga trådlösa sändtagare som integrerade kretsar (integrated circuits, IC). Denna avhandling tar itu med de största utmaningarna inom design av bredbandiga trådlösa sändtagar integrerade kretsar. Avhandlingen är indelad i två delar. Del I beskriver design av viktiga kretsblock så som väldigt selektiva bredbandiga radiofrekvens (RF) front-ends och integrerade test moduler som typiskt finns i trådlösa mottagare. Design av höghastighets, kapacitiva digital-till-analog omvandlare (digital-to-analog converter, DAC) för trådlösa sändare återfinns i Del II.

Det första bidraget i Del I är design och implementation av ett bredbandigt RF frontend i 65-nm CMOS. För att nå en blocker rejection jämförbart med surface-acousticwave (SAW) filter har RF mottagren hög selekterbarhet och inställbarahet och använder impedanstransformerings filtrering tillsammans med en tvåstegs arkitektur. Det är välkänt att lågbrusiga förstärkare (low-noise amplifier, LNA) vilket utgör det första front-end steget till stor del bestämmer prestandan av mottagaren i termer av noise figure (NF) och linjäritet (IIP3/P1dB). Den föreslagna LNA:n använder dubbel korskopplingsteknik för att reducera NF och komplementär deriverings superposition (DS) samt resisitiv återkoppling används för att få hög linjäritet. Den resisitiva återkopplingen förbättrar också insignalsmatchningen. I mätningar visade sig mottagarens prestanda vara jämförbar med SAW filter och gav en blocker rejection högre än $38 \mathrm{~dB}$, NF 3.2-5.2 dB, utanför band IIP3 > +17 dBm och blocker P1dB > +5 $\mathrm{dBm}$ för frekvensspannet $0.5-3 \mathrm{GHz}$. 
Det andra bidraget i Del II är design av en RF amplitud detektor för testning i den integrerade kretsen. När komplexiteten av RF integrerade kretsar ökar, blir testning samt felsökning allt mer krävande. Degradering av prestandan eller drift från den optimala arbetspunkten kan orsaka systemhaveri. För att undvika detta och garantera acceptabel prestanda över process-, spännings- och temperaturvariationer har test och kalibrering av RF integrerade kretsar blivit oumbärliga. En bredbandig RF amplitud detektor med stort dynamiskt omfång har designats för att möjliggöra testning i kretsen föreslås. Förstärknings förbättring och subranging tekniker används i detektorn för att öka förstärkningen för alla insignals amplituder utan att kompromissa på inimpedansen. En kretsteknik lämplig för integrerad testning av IP3/IP2 i integrerade RF detektorer introduceras också.

Del II består av design och analys av en höghastighets switchad kapacitans (switched capactior, SC) digital-till-analog omvandlare för $60-\mathrm{GHz}$ radiosändare. DAC:ar är ett fundamentalt byggblock i radiosändare, SC DAC:ar har flera fördelar mot strömstyrnings DAC arkitekturer. Speciellt möjliggör den goda kapacitansmatchningen SC DAC:ar att nå högre linjäritet. Switcharna i DAC:en realiseras av MOS transistorer som används i triode regionen vilket radikalt minskar kravet på hög matningsspänning. Därför kan SC DAC:ar implementeras med lägre matningsspänning i avancerade CMOS processer jämfört med strömstyrnings DAC:ar. Det första bidraget i Del II består av en analys av faktorerna som begränsar prestandan i kapacitiva pipelineade DAC:ar. Där visas hur DAC:ens prestanda till stor del begränsas av klockgenomslag och insvängningseffekter i SC arrayerna medans påverkan av kapacitans mismatch och kT/C brus är försumbara. Baserad på denna analys föreslås i det andra bidraget i Del II en split-segmented SC array DAC för att överkomma problemen med klockgenomslag eftersom denna topologi eliminerar laddningspropagering i pipelinen. Implementerad i 65-nm CMOS, åstadkommer den här 12-bit SC DAC:en en SFDR på mer än $44 \mathrm{~dB}$ inom signal bandbredden av $1 \mathrm{GHz}$ med integrerat minne för digital data generering. Effektförbrukningen är $50 \mathrm{~mW}$ med en $1.2 \mathrm{~V}$ matningsspänning. Liknande prestanda fås också med en lägre matningsspänning på $0.9 \mathrm{~V}$ vilket visar på skalbarheten av SC DAC:ar i mer avancerade CMOS processer. Dessutom tillgodoser den föreslagna SC DAC:en spektrum masken för IEEE 802.11ad WiGig standarden med ett andra ordningens rekonstruktionsfilter och är därför lämplig att användas för basbandet i 60$\mathrm{GHz}$ radio. 


\section{Preface}

This Ph.D thesis presents my research work during the period January 2011 to January 2016 at the Division of Integrated Circuits and Systems (EKS), Department of Electrical Engineering (ISY), Linköping University, Sweden. I have spent 4 years (80\% of the total 5 years) on research and course work of at least 90 Europen Credit Transfer System (ECTS). The $20 \%$ left which is 1 year is used for full-time teaching duties. The dissertation is mainly based on the following peer-reviewed journal articles and conference publications:

- Paper 1 - Quoc-Tai Duong, Fahad Qazi, and Jerzy J. Dabrowski, “Analysis and Design of Low Noise Transconductance Amplifier for Selective Receiver Frontend," Journal of Analog Integrated Circuits and Signal Processing, vol. 85, no. 2, pp. 361-372, Nov. 2015.

- Paper 2 - Fahad Qazi, Quoc-Tai Duong, and Jerzy J. Dabrowski, "Two-Stage Highly Selective Receiver Front-End Based on Impedance Transformation Filtering," IEEE Transactions on Circuits and Systems II: Express Briefs (TCASII), vol. 62, no. 5, pp. 421-425, May. 2015.

- Paper 3 - Quoc-Tai Duong, and Jerzy J. Dabrowski, "Wideband RF Detector Design for High Performance On-Chip Test," IEEE Norchip, Copenhagen, Danmark, pp. 1-4, Nov 2012.

- Paper 4 - Quoc-Tai Duong, and Jerzy J. Dabrowski, "Focused calibration for advanced RF test with embedded RF detectors," IEEE European Conference on 
Circuit Theory and Design (ECCTD), Dresden, Germany, pp. 1-4, September 2013.

- $\quad$ Paper 5 - Quoc-Tai Duong, Jerzy J. Dabrowski, and Atila Alvandpour, "Design and analysis of high speed capacitive pipeline DACs," Journal of Analog Integrated Circuits and Signal Processing, vol. 80, no. 3, pp. 359-374, Sept. 2014.

- Paper 6 - Quoc-Tai Duong, Ameya Bhide, and Atila Alvandpour, "A 1-GHz Bandwidth 12-bit SC DAC for 60-GHz Radio in 65-nm CMOS," Manuscript to be summited to IEEE journal.

- Paper 7 - Fahad Qazi, Quoc-Tai Duong, and Jerzy J. Dabrowski, "Tunable Selective Receiver Front-End with Impedance Transformation Filtering," Journal of Circuit Theory and Applications, August 2015, DOI: 10.1002/cta.2125.

- Paper 8 - Quoc-Tai Duong, and Jerzy J. Dabrowski, "Low Noise Transconductance Amplifier Design for Continuous-Time delta sigma Wideband Frontend," IEEE European Conference on Circuit Theory and Design (ECCTD), Linkoping, Sweden, pp. 825-828, August 2011.

- Paper 9 - Quoc-Tai Duong, Jerzy J. Dabrowski, and Atila Alvandpour, "Highly linear open-loop output driver design for high speed capacitive DACs," IEEE Norchip, Vilnius, Lithuania, pp. 1-4, Nov 2013.

The following papers were also published during the period of my Ph.D. They are not included in this thesis:

- Fahad Qazi, Quoc-Tai Duong, and Jerzy J. Dabrowski, "Wideband RF Frontend Design for Flexible Radio Receiver," IEEE International Symposium on Integrated Circuits (ISIC), Singapore, pp. 220-223, December. 2011.

- Fahad Qazi, Quoc-Tai Duong, and Jerzy J. Dabrowski, "Blocker and Image Reject Low-IF Frontend," IEEE European Conference on Circuit Theory and Design (ECCTD), Dresden, Germany, pp. 1-4, September 2013.

- Kairang Chen, Quoc-Tai Duong, and Atila Alvandpour, "Power Analysis for TwoStage High Resolution Pipeline SAR ADC," IEEE Mixed Design of Integrated Circuits \& Systems (MIXDES), Torun, Poland, pp. 496-499, June. 2015. 
- Rengarajan Ragavan, Anand Narayanan, Mikael Bengtsson, and Quoc-Tai Duong, "A 0.35um CMOS 6-bit Current Steering DAC," IEEE European Conference on Circuit Theory and Design (ECCTD), Dresden, Germany, pp. 1-4, September 2013.

- Quoc-Tai Duong, and Jerzy J. Dabrowski, "Design of Low Noise Transconductance Amplifier for Current-Mode Wideband RF Frontend," Swedish System-on-Chip Conference (SSOCC), Sponsored by IEEE SSCS-Sweden Chapter, 2011.

- Quoc-Tai Duong, and Jerzy J. Dabrowski, "On-chip IP3/IP2 Advanced RF Test and Calibration Technique with Embedded RF Detectors," Swedish System-onChip Conference (SSOCC), Sponsored by IEEE SSCS-Sweden Chapter, 2013. 


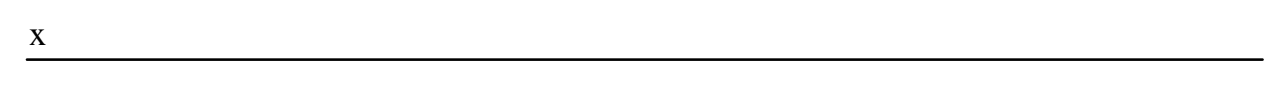




\section{Contributions}

The main contributions of this dissertation are as follows:

- Design and analysis of low noise trans-conductance amplifier (LNTA) are presented. It is implemented in a selective receiver front-end based on impedance transformation filtering in $65 \mathrm{~nm}$ CMOS technology. The measured results which achieve high blocker rejection, high IIP3 and low NF put the design among the state-of-the-art reported works.

- Design of RF detector and development of a technique suitable for on-chip IP3/IP2 $\mathrm{RF}$ test are presented. They are used for on-chip measurement of device under test (DUT) gain, P1dB, and IIP3.

- Design and analysis of high speed capacitive pipeline digital-to-analog converters (DACs). The analyses show the main limiting factors of high speed pipeline switched-capacitor (SC) array and feasibility of designing a SC DAC at GHz frequency.

- Based on the above analysis of pipeline DAC, a split-segmented SC DAC architecture is proposed in order to overcome the clock feed-through problem since this topology eliminates pipelined charge propagation. Implemented in $65 \mathrm{~nm}$ CMOS technology, the 12-bit SC DAC prototype achieves highest signal bandwidth $(1 \mathrm{GHz})$ among recent reported SC DACs, to the authors' knowledge. Furthermore it satisfies the spectral mask of the IEEE 802.11ad WiGig standard with a second-order reconstruction filter so it can be used for $60-\mathrm{GHz}$ radio baseband. 


\section{Abbreviations}

$\begin{array}{ll}\text { BP } & \text { Band Pass } \\ \text { BR } & \text { Blocker Rejection } \\ \text { BW } & \text { Band Width } \\ \text { CMOS } & \text { Complementary Metal-Oxide-Semiconductor } \\ \text { CR } & \text { Cognitive Radio } \\ \text { CT } & \text { Continuos Time } \\ \text { CUT } & \text { Circuit Under Test } \\ \text { D/A } & \text { Digital-to-Analog Converter } \\ \text { DAC } & \text { Digital-to-Analog Converter } \\ \text { DFT } & \text { Discrete Fourier Transform } \\ \text { DR } & \text { Dynamic Range } \\ \text { DS } & \text { Derivative Superposition } \\ \text { DUT } & \text { Device Under Test } \\ \text { EVM } & \text { Error Vector Magnitude } \\ \text { FDD } & \text { Frequency Division Duplex } \\ \text { FFT } & \text { Fast Fourier Transform } \\ \text { FOM } & \text { Figure-Of-Merit }\end{array}$


FPGA Field-Programmable Gate Array

FS

Full Scale

GSM

Global System for Mobile communication

$\mathrm{HP}$

High Pass

IC

Integrated Circuit

IEEE

The Institute of Electrical and Electronics Engineers

IF

Intermediate Frequency

IIP2

Second order Input Intercept Point

IIP3

Third order Input Intercept Point

LNA

Low Noise Amplifier

LNTA

Low Noise Trans-conductance Amplifier

LO

Local Oscillator

LP

Low Pass

LTE

Long Term Evolution

$\mathrm{MC}$

Monte Carlo

MOS

Metal-Oxide-Semiconductor

$\mathrm{NF}$

Noise Figure

NMOS

N-channel Metal-Oxide-Semiconductor

NTF

Noise Transfer Function

OBI

Out-of-band Interference/Interfere

OFDM

Orthogonal Frequency Division Multiplexing

P1dB

$1 \mathrm{~dB}$ Compression Point

PA

Power Amplifier

PCB

Printed Circuit Board

PMOS

P-channel Metal-Oxide-Semiconductor

PVT

Process-Voltage-Temperature

RF

Radio Frequency

$\mathrm{RX}$

Receiver

SAW

Surface Acoustic Wave 
SC

SD

SDR

SFDR

SNR

SSB

STF

$\mathrm{T} / \mathrm{H}$

TIA

TX

UMTS

UWB

WLAN

WPAN

$\Delta \sum$
Switched Capacitor

Standard Deviation

Software-Defined Radio

Spurious Free Dynamic Range

Signal-to-Noise ratio

Single Sideband

Signal Transfer Function

Track-and-Hold

Transimpedance Amplifier

Transmitter

Universal Mobile Telecommunications System

Ultra-Wideband

Wireless Local Area Network

Wireless Personal Area Network

Delta-Sigma 
$\underline{x v i}$ 


\section{Acknowledgments}

I am very grateful and would like to say many thanks for those who give me the help, support, and encouragement. They are my family members, teachers, colleagues, and friends. I would like to thank the following people who help and support my study:

- My supervisor and advisor Prof. Atila Alvandpour, for his guidances, advices, and supports. Thanks for giving me the opportunity to pursue this study to be able to become a Ph.D.

- $\quad$ Associate Prof. Jerzy J. Dąbrowski for supporting technical discussions and helping to write several papers.

- Dr. Prakash Harikumar for his help to improve English writing of thesis.

- Dr. Amin Ojani for providing the Word and PowerPoint templates for this thesis.

- Dr. Ameya Bhide for technical discussions.

- Docent J. Jacob Wikner for his tips on use of cadence and scripting.

- Adj. Prof. Ted Johansson for the guide of $28 \mathrm{~nm}$ CMOS simulation.

- Dr. Jonas Fritzin for giving suggestion about parasitic reduction.

- M.Sc. Martin Nielsen Lönn for preparing the "sammanfattning" of this thesis.

- Dr. Ngo Quoc Hien for helping me in initial set-up of my Ph.D student life.

- All the past and present members of Division of Integrated Circuits and Systems and friends, especially Prof. emeritus Christer Svensson, Docent Behzad Mesgarzadeh, Dr. Fahad Qazi, Dr. Christer Jansson, Dr. Timmy Sundström, Dr. Mostafa Osgooei, Dr. Pablo Viana Da Silva, Dr. Dai Zhang, Dr. Mark Vesterbacka, Dr. Mikael Olofsson, Dr. Armin Jalili, Lecturer Sivert Lundgren, M.Sc. Omid 
Najari, M.Sc. Daniel Svärd, M.Sc. Kairang Chen, M.Sc. Vishnu Unnikrishnan, Dr. Muhammad Irfan Kazim, Dr. Tomas Jonsson, Dr. Nadeem Afzal, M.Sc. Joakim Alvbrant, Dr. Anu Kalidas M. Pillai, Lic. Muhammad Touqeer Pasha. Thanks for creating such a friendly environment.

- Research engineer Arta Alvandpour for all his help with the equipment and hardware issues.

- Our technical supports Joakim Olovsson, Thomas Johansson, and Jean-Jacques Moulis for solving all computer related issues.

- $\quad$ Our current and past secretaries Gunnel Hässler, Maria Hamner and Anna Folkeson for taking care of all administrative issues. Department administrator Susanna von Sehlen for uploading course credits and providing transcripts.

- My parents and my siblings for giving me unlimited love and support.

- My wife M.Sc. Tran Mai Phuong and daughter Duong Tran Marie give me the happiness and motivation of life as well as encourage for overcoming difficulties.

Duong Quoc Tai

Linköping, January 2016 


\section{Contents}

Abstract iii

Sammanfattning v

Preface vii

Contributions xi

Abbreviations xiii

Acknowledgments $\quad$ xvii

Contents $\quad$ xix

List of Figures $\quad$ xxiii

List of Tables $\quad$ xxvii

Chapter 1 Introduction 1

1.1 Overview 1

1.2 Part I: RF Receiver front-end and design for on-chip test on receiver side 2

1.3 Part II: High Speed SC DACs for wideband wireless transmitters 5

$\begin{array}{lll}1.4 & \text { Thesis Organizations } & 7\end{array}$ 


\section{Chapter 2 Design Considerations of RF Receiver Front-} end, Testability and high speed SC DACs 9

2.1 Introduction 9

2.2 Design considerations of RF receivers 10

$\begin{array}{ll}2.2 .1 \text { Sensitivity and noise figure } & 10\end{array}$

$\begin{array}{ll}2.2 .2 \text { Selectivity } & 10\end{array}$

2.2.3 Nonlinearity and Intermodulation 10

2.2.4 Dynamic range 11

2.3 Design considerations of RF LNAs 11

2.3.1 Noise figure (NF) 11

$\begin{array}{ll}2.3 .2 \text { Gain } & 14\end{array}$

$\begin{array}{ll}2.3 .3 \text { Input return loss } & 15\end{array}$

$\begin{array}{ll}2.3 .4 \text { Stability } & 15\end{array}$

$\begin{array}{ll}2.3 .5 \text { Linearity } & 15\end{array}$

$\begin{array}{ll}2.3 .6 \text { Bandwidth } & 18\end{array}$

$\begin{array}{lll}2.3 .7 & \text { Power dissipation } & 18\end{array}$

$\begin{array}{ll}2.3 .8 \text { Input matching } & 18\end{array}$

$\begin{array}{ll}2.3 .9 \text { Reverse isolation } & 18\end{array}$

2.4 The considerations of design for on-chip test 18

$\begin{array}{ll}2.4 .1 \text { Input impedance of RF detectors } & 18\end{array}$

$\begin{array}{ll}2.4 .2 \text { Area of on-chip test system } & 19\end{array}$

2.4.3 Wide bandwidth of RF detectors 19

2.4.4 Power dissipation of on-chip test system 19

2.4.5 Gain and dynamic range of RF detectors 19

2.5 Design considerations of high speed capacitive DACs for wideband wireless transmitters 19

$\begin{array}{ll}2.5 .1 \text { The } \mathrm{kT} / \mathrm{C} \text { nosie } & 19\end{array}$

2.5.2 Capacitor mismatch 20

2.5.3 Settling time 20

2.5.4 Clock feed-through effect 20

$\begin{array}{ll}\text { 2.5.5 Switching noise } & 21\end{array}$

$\begin{array}{ll}2.5 .6 \text { Output driver } & 21\end{array}$

Part I Wideband RF Receiver Front-end and Design for On-chip Test on Receiver Side 23

Chapter 3 Design of RF Receiver Front-end 25

3.1 Introduction 25

3.2 Design of Two-Stage Receiver Front-end 26 
3.3 LNA Design 28

3.4 LNA Noise Analysis 31

3.5 LNA Linearity Analysis Using Volterra Series 36

3.6 Implementation of a RF Selective Receiver Front-end 42

Chapter 4 Wideband RF Detector Design for On-Chip Test 45

4.1 Introduction 45

4.2 RF Test Setup 46

4.3 RF Detector Design 48

4.4 RF Detector for Two-tone Test 50

4.5 IP3/IP2 Test Technique 51

4.6 Simulation Results 54

Part II Design of High Speed SC DACs for 60-GHz radio baseband on Transmitter Side 59

Chapter 5 Design and Analysis of High Speed Capacitive Pipeline DACs 61

5.1 Introduction 61

5.2 Capacitive DAC Architecture 62

5.3 Capacitive pipeline Array Analysis 64

5.3.1 Nosie analysis 64

$\begin{array}{ll}\text { 5.3.2 Capacitor mismatch analysis } & 67\end{array}$

5.3.3 Clock feed-through effect 69

5.3.4 Settling time analysis $\quad 69$

5.3.5 Bounds for unit capacitor $\quad 72$

5.4 Output Driver Design $\quad 74$

5.4.1 Problem and solution $\quad 74$

5.4.2 Volterra series model $\quad 76$

5.4.3 Thermal noise analysis $\quad 80$

5.5 Simulation Results 80

Chapter 6 A 1-GHz Bandwidth 12-bit SC DAC for 60-GHz Radio in 65-nm CMOS 87

$\begin{array}{lll}6.1 & \text { Introduction } & 87\end{array}$ 
6.2 A 12-bit Split-segmented SC DAC Architecture 89

6.3 The Analysis of kT/C Noise and Capacitor Mismatch in SC Array

6.3.1 Thermal noise analysis

6.3.2 Capacitor mismatch analysis 97

6.4 Settling Time Analysis and Parasitic Effects 99

6.4.1 Settling time analysis 99

6.4.2 Capacitance parasitic effects 101

6.5 Switching Noise 101

6.6 Output Driver Design and Analysis 104

6.6.1 Volterra series model 106

6.6.2 Simulation results 107

6.7 Chip Implementation and Measurement Results 108

Chapter 7 Conclusions and Future Works 117

$\begin{array}{lll}7.1 \text { Conclusions } & 117\end{array}$

$\begin{array}{lll}7.2 & \text { Future Works } & 120\end{array}$

Appendix

123

$\begin{array}{ll}\text { References } & 135\end{array}$

$\begin{array}{ll}\text { Paper Collections } & 145\end{array}$ 


\section{List of Figures}

Figure 1.1: Conceptual diagram of the low-pass blocker filtering (current-mode receiver) in [11].

Figure 1.2: (a) DS technique with dual-NMOS. (b) Third-order distorion terms of the main transistor $\left(\mathrm{g}_{3 \mathrm{~A}}\right)$, auxiliary transistor $\left(\mathrm{g}_{3 \mathrm{~B}}\right)$, and total output $\left(\mathrm{g}_{3}\right)$ in $[20] \ldots \ldots \ldots \ldots \ldots . . . . . . . . .$.

Figure 1.3: Capacitive cross-coupling technique in [28] ....................................... 5

Figure 1.4: Spectral mask of WiGig for single channel operation in [44] ...................... 6

Figure 1.5: $60 \mathrm{GHz}$ Band Channel Plan and Frequency Allocation by Region in [44]... 6

Figure 2.1: Effect of intermodulation from two blockers on wanted signal. ................ 11

Figure 2.2: Thermal noise of resistor a) Thevenin b) Norton. ...................................... 12

Figure 2.3: Noise models of MOSFET (a) current source (b) voltage source. .............. 12

Figure 2.4: The input-referred noise voltage model of LNA..................................... 14

Figure 2.5: (a) Conventional DS technique. (b) Third-order distorion terms of the main transistor (g3A), auxiliary transistor (g3B), and total output (g3)............................. 21

Figure 3.1: Architecture of selective two-stage RF front-end [16] .............................. 27

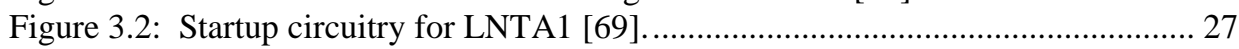

Figure 3.3: Circuit schematic of LNTA2 [69]...................................................... 27

Figure 3.4: LNTA complementary DS architectures, a) common source b) common

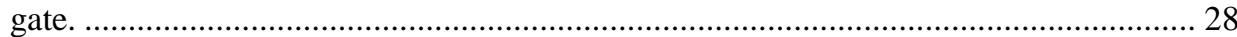

Figure 3.5: Differential LNTA implementing DS and capacitive cross-coupling

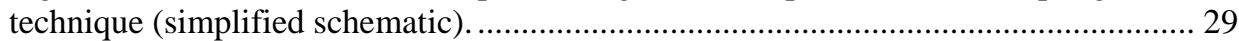

Figure 3.6: S11 and linearity improvement by resistive source degeneration. .............. 30

Figure 3.7: Circuit schematic of proposed wideband LNTA................................... 31

Figure 3.8: LNTA circuit for noise analysis. ........................................................ 32

Figure 3.9 NF comparison of analytical model (3.13-3.19) and SpectreRF® circuit simulation for proposed LNTA (transistor level). ................................................... 35 
Figure 3.10 a) Schematic of conventional inverter, b) Simulation of third-order

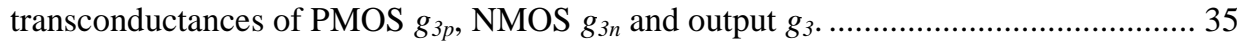
Figure 3.11: a) Schematic of resistive-feedback technique, b) Simulation of third-order

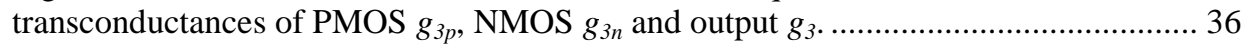
Figure 3.12: Equivalent circuit of a proposed wideband LNTA................................ 38 Figure 3.13: The third-order voltage gain $H_{3}(3.42)$ versus the bias voltage $V_{g s n} \ldots \ldots \ldots . . .40$ Figure 3.14: IIP3 comparison of analytical expression (3.43) and SpectreRF® simulation for LNTA, using two-tone $40 \mathrm{MHz}$ spacing (transistor level). ..................... 41 Figure 3.15: Monte-Carlo simulation of LNTA IIP3 obtained with 50 iterations at $f_{R F}=$

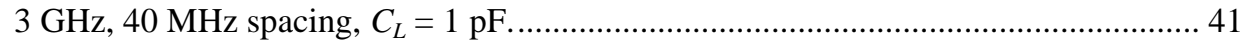

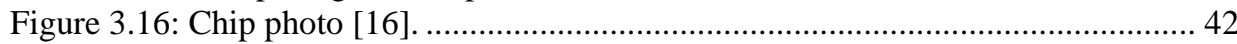

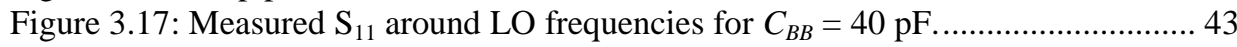

Figure 4.1: On-chip test setup for wideband SAW-less receiver................................. 47

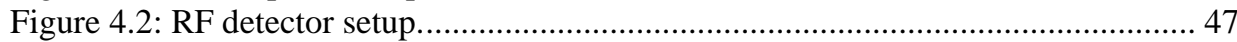

Figure 4.3: Response of RF detector to DC and one-tone input.............................. 48

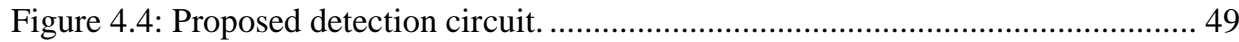

Figure 4.5: Two-bit bias circuit for sub-ranging operation....................................... 49

Figure 4.6: RF detector characteristics for two-tone input with IM3 tones................. 51

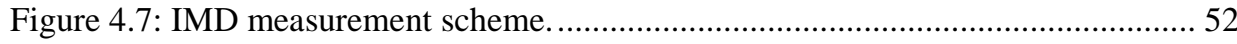

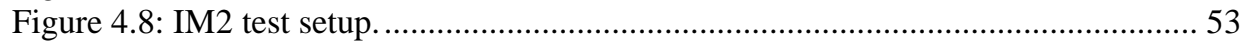

Figure 4.9: Detector characteristic using sub-ranges (a), and in Range 1 (b).............. 54

Figure 4.10: Comparison detector signal before and after VGA. ................................. 54

Figure 4.11: Output voltage difference compared to $0.5 \mathrm{GHz}$ operation....................... 55

Figure 4.12: The input impedance vs. frequency.................................................. 55

Figure 4.13: LNA spectrum for unloaded output and $P_{\text {in }}=-35 \mathrm{dBm}$........................ 57

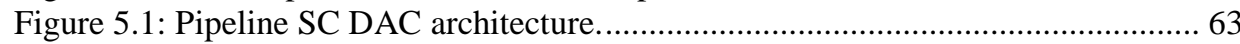

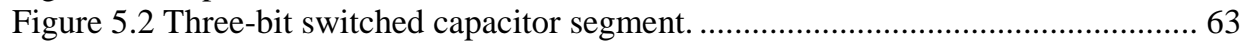

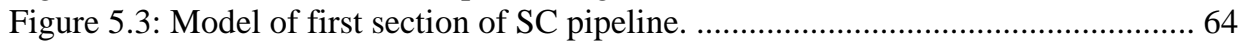

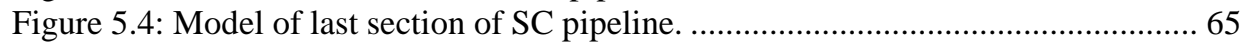

Figure 5.5: SC noise PSD comparison of analytical model (5.7) and SpectreRF ${ }^{\mathrm{TM}}$

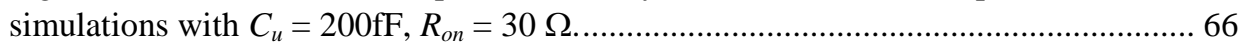

Figure 5.6: $\mathrm{V}_{\mathrm{DNL}}$ standard deviation comparison of estimated model (5.18) and SpectreRF ${ }^{\mathrm{TM}} \mathrm{MC}$ simulations against 1/6 LSB voltage of 12 bit DAC.

Figure 5.7: Charged and discharged circuits: (a) Leakage due to off-resistance of data switches, (b) Incomplete charge due to on-resistance of data switches (c) Incomplete charge redistribution due to inbetween switches. .................................................. 70 Figure 5.8: Off-resistance and on-resistance of T-gate switch vs. width in 65nm CMOS

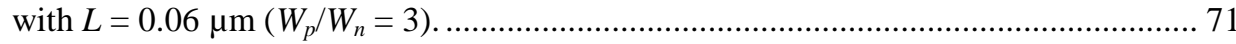
Figure 5.9: Design area W-C $\mathrm{C}_{\mathrm{u}}$ for 7-bit and 8-bit SC DAC, $f_{S}=3 \mathrm{GHz}, K_{d i f}=20 \%$ and

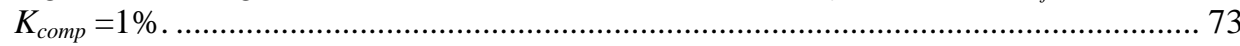
Figure 5.10: Design area W-C $\mathrm{C}_{\mathrm{u}}$ for 7-bit SC DAC with different clock frequency $\left(f_{s}\right)$

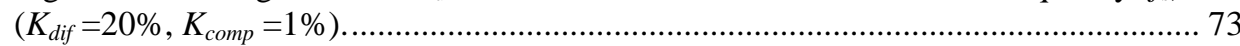

Figure 5.11: Design area $\mathrm{W}-\mathrm{C}_{\mathrm{u}}$ for 6-bit $\mathrm{SC}$ DAC at $12 \mathrm{GHz}$ clock frequency............. 74 
Figure 5.12: (a) DS technique with dual-NMOS. (b) Third-order distorion terms of the main transistor $\left(\mathrm{g}_{3 \mathrm{~A}}\right)$, auxiliary transistor $\left(\mathrm{g}_{3 \mathrm{~B}}\right)$, and total output $\left(\mathrm{g}_{3}\right)$ using ST 65nm CMOS process, $(W / L)_{\mathrm{MA}}=20 / 0.065 \mu \mathrm{m},(W / L)_{\mathrm{MB}}=17 / 0.065 \mu \mathrm{m}, V_{d s}=1.2 \mathrm{~V}$ and $V_{\text {shift }}=$ $282 \mathrm{mV}$.

Figure 5.13: (a) DS technique with resistive feedback. (b) Third-order distorion terms of the main transistor $(\mathrm{g} 3 \mathrm{~A})$, auxiliary transistor $\left(\mathrm{g}_{3 \mathrm{~B}}\right)$, and total output $\left(\mathrm{g}_{3}\right)$ using ST $65 \mathrm{~nm}$ CMOS process, $(W / L)_{\mathrm{MA}}=20 / 0.065 \mu \mathrm{m},(W / L)_{\mathrm{MB}}=17 / 0.065 \mu \mathrm{m}, V_{d s}=1.4 \mathrm{~V}$ and $V_{\text {shift }}$ $=452 \mathrm{mV}$. 76

Figure 5.14: Comparison of conventional DS technique and DS technique combined with resistive feedback.

Figure 5.15: Comparison conventional (CS) and proposed DS technique with resistive

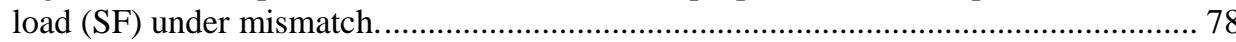

Figure 5.16: Equivalent circuit of the proposed linear output driver. .......................... 79 Figure 5.17: HD3 comparison of Volterra series model (5.38) and SpectreRF circuit

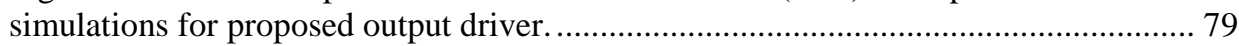

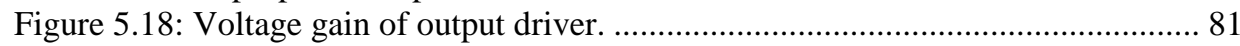

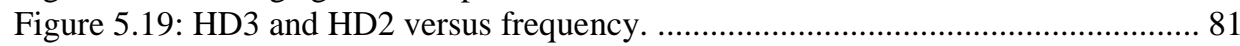

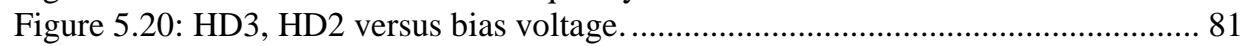

Figure 5.21: HD3and HD2 versus temperature. ....................................................... 82

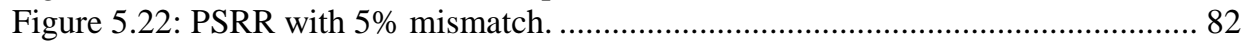

Figure 5.23: SFDR against clock frequency for 7-bit and 8-bit $(\mathrm{OSR}=1.1)$.............. 83

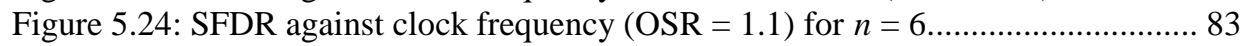

Figure 6.1: The 12-bit split-segmented SC DAC architecture.................................... 89

Figure 6.2: The Mux-based 6-63 decoder................................................................. 91

Figure 6.3: Model of thermometer switch noise. ...................................................... 92

Figure 6.4: Model of switch noise for the first binary segment. ................................. 93

Figure 6.5: Model of switch noise for the second binary segment. ............................ 94

Figure 6.6: SC noise PSD comparison of analytical model (6.20) and SpectreRFTM

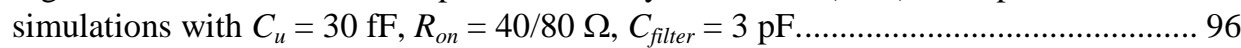

Figure 6.7: SC PSD comparison of kT/C noise (6.20) with $R_{o n}=40 \Omega, 80 \Omega$ and quantization noise $(6.21), \mathrm{f}_{\mathrm{s}}=3 \mathrm{GHz}, \mathrm{V}_{\mathrm{FS}}=0.8 \mathrm{~V}, \mathrm{C}_{\text {filter }}=3 \mathrm{pF}, \mathrm{n}=12$.

Figure 6.8: $\mathrm{V}_{\mathrm{DNL}}$ standard deviation comparison of estimated model (6.27) and

SpectreRFTM MC simulations against 1/6 LSB voltage of 12 bit DAC . ...................... 98

Figure 6.9: Equivalent circuit model for off-resistance. .......................................... 100

Figure 6.10: Equivalent circuit model for capacitance parasitic................................ 101

Figure 6.11: (a) Wire-bonding model. (b) Seprating supplies to reduce noise............ 102

Figure 6.12: Damping/filtering noise on signal paths............................................ 103

Figure 6.13: (a) Source-follower buffer. (b) Second and third-order distorion terms of

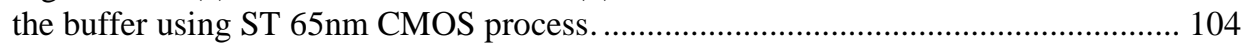

Figure 6.14: (a) DS technique in SF buffer. (b) Third-order distorion terms of the main transistor (g3n), auxiliary transistor (g3p), and total output (g3). (c) Second-order distorion terms of the main transistor $(\mathrm{g} 2 \mathrm{n})$, auxiliary transistor $(\mathrm{g} 2 \mathrm{p})$, and total output (g2) using ST 65nm CMOS process. 
Figure 6.15: Equivalent circuit of the proposed linear output driver. ......................... 106

Figure 6.16: HD3 comparison of analytical expressions (6.44) and Spectra RF

simulations for the proposed output driver.

106

Figure 6.17: HD3 and HD2 versus frequency with 5\% mismatch............................... 107

Figure 6.18: HD3 and HD2 versus bias voltage with 5\% mismatch. .......................... 108

Figure 6.19: HD3 and HD2 versus temperature with 5\% mismatch........................... 108

Figure 6.20: Measurement setup of DAC with on-chip memory............................... 109

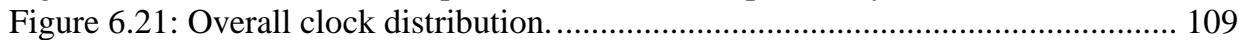

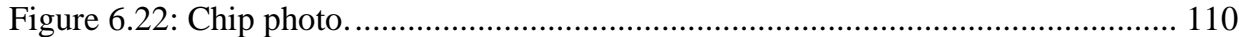

Figure 6.23: Measured $44 \mathrm{~dB}$ SFDR with a $1 \mathrm{GHz}$ single tone at $5 \mathrm{GS} / \mathrm{s}$. .................. 110

Figure 6.24: Measured $64 \mathrm{~dB}$ SFDR with a $16 \mathrm{MHz}$ single tone at $1 \mathrm{GS} / \mathrm{s}$................ 111

Figure 6.25: Measured IM3 of $-38 \mathrm{dBc}$ with two tones at $5 \mathrm{GS} / \mathrm{s}$. ............................ 111

Figure 6.26: Measured SFDR and IM3 versus frequency at $5 \mathrm{GS} / \mathrm{s} . \ldots \ldots \ldots \ldots \ldots \ldots \ldots \ldots . . . \ldots 112$

Figure 6.27: Measured $40 \mathrm{~dB}$ SFDR with a $1 \mathrm{GHz}$ single tone at $5 \mathrm{GS} / \mathrm{s}$ with $0.9 \mathrm{~V}$

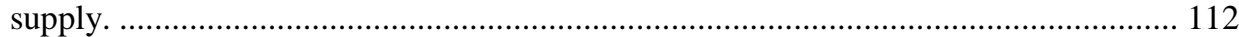

Figure 6.28: Measured $64 \mathrm{~dB}$ SFDR with a $16 \mathrm{MHz}$ single tone at $1 \mathrm{GS} / \mathrm{s}$ with $0.9 \mathrm{~V}$

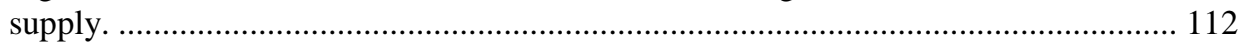

Figure 6.29: Measured IM3 of $-35 \mathrm{dBc}$ with two tones at $5 \mathrm{GS} / \mathrm{s}$ with $0.9 \mathrm{~V}$ supply.. 113

Figure 6.30: Measured SFDR versus frequency at $5 \mathrm{GS} / \mathrm{s}$ with $1.2 \mathrm{~V}$ and $0.9 \mathrm{~V}$ supply.

Figure 6.31: Measured spectral mask with 16-QAM single-carrier random data at 5.28

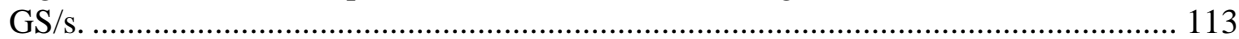

Figure 7.1: Conventional architecture used in [47, 49-50] ...................................... 120

Figure 7.2: High speed Nyquist DAC based architecture proposed in [48] ................ 120

Figure 7.3: $\triangle \sum \mathrm{DAC}$ proposed in [108] can use SC DAC cells instead of current-steering

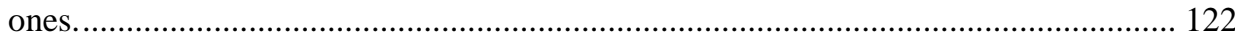




\section{List of Tables}

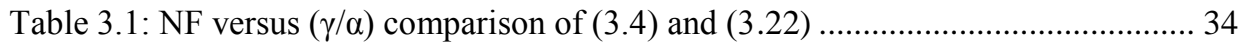

Table 3.2: Performance comparison of LNTA ......................................................... 43

Table 3.3: Performance comparison of Receiver Front-end ........................................ 44

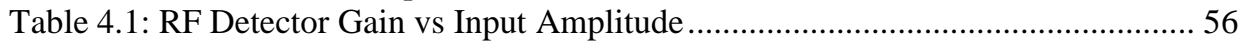

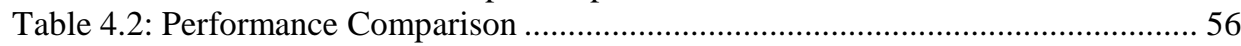

Table 4.3: Comparison of IIP3 Measurement Techniques ......................................... 58

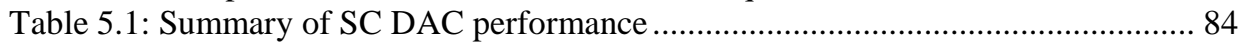

Table 5.2: Performance comparison of SC DACs .................................................... 85

Table 6.1: The truth table for 3-8 decoder: Mux (a), 2-4 Select generator (b)............... 90

Table 6.2: The truth table for 6-63 decoder: Mux (a), 3-8 Select generator (b)............. 91

Table 6.3: HD2 and HD3 versus different corners with 5\% mismatch.......................... 108

Table 6.4: Power and Area Breakdown of the DAC by Function .............................. 111

Table 6.5: Comparison with other DACs for the same application of 60-GHz Radio. 115

Table 6.6: Comparison of This Work With State-of-the-art Reported DACs ............. 116 


\section{Chapter 1}

\section{Introduction}

\subsection{Overview}

As mentioned in the abstract this dissertation focuses to solve the key challenges of the wideband wireless transceivers. It is divided into 2 parts. Part I of thesis is design of wideband RF receiver front-end and design for on-chip test on receiver side. The contributions of author in this part are design of wideband RF LNA and collaborating for designing and implementing receiver front-end (Chapter 3). Author also designs RF detector for on-chip test (Chapter 4). Part II of thesis is design and analysis of wideband SC DACs for $60-\mathrm{GHz}$ radio baseband on transmitter side (Chapter 5, 6). This Chapter elaborates upon the design challenges and applications for wideband receiver front-ends, on-chip test blocks and high speed SC DACs for wireless transmitters. 


\subsection{Part I: RF Receiver front-end and design for on- chip test on receiver side}

The first work in Part I is RF receiver front-end. The so called SAW-less RF receivers have been thoroughly investigated recently addressing needs of the modern wireless systems. Using the concept of $N$-path filter [1] several designs of tunable RF filters in CMOS technology have been presented [2]-[10]. In principle, it is the passive mixer transparency that enables simultaneous signal down- and up-conversion necessary in this case. For a low-pass impedance at baseband, the up-converted voltage signal appears band-limited accordingly that can be thought as impedance transformation in frequency from baseband to RF. Selectivity, achieved in this way, presents high $\mathrm{Q}$ factors which are attractive in RF filtering. In effect, filters designed using this technique are good candidates to replace inflexible SAW filters, in particular, in software defined- or cognitive radio (SDR/CR) applications. However, as the rejection of one such a filter is usually less than $20 \mathrm{~dB}$, using another filter section or a more sophisticated baseband impedance can be necessary in a SAW-less scenario to suppress interference and avoid significant intermodulation effects or gain compression. Resilience to out-of-band blockers, in extreme cases up to $0 \mathrm{dBm}$ at antenna input, is the main challenge in this case while maintaining noise figure and intermodulation performance over the wide range of frequencies used in personal and data communication systems.

For a multi-standard radio receiver the wideband RF front-end circuit is essential. It is well known that a low-noise amplifier (LNA) as the first front-end stage largely decides the receiver performance in terms of noise figure (NF) and linearity. With relaxed requirements on RF filters the demands placed on the front-end linearity are usually increased according to intermodulation or cross-modulation effects evoked by strong interferers. While the nonlinear contribution of the following receiver stages is raised by the LNA gain, the overall NF is reduced. As a consequence a reasonable balance between linearity and noise performance of the LNA, mixer, and to some extent the baseband stages must be attained. One possible solution to this problem is a currentmode front-end where LNA is a transconductance amplifier (LNTA) followed by a passive mixer [10-16] as shown in Fig. 1.1. Since current rather than a voltage is applied, the mixer design is simplified and also the effect of $1 / \mathrm{f}$ noise is diminished. Most of those designs implement the concept of so called SAW-less front-end making use of N-path filtering [17]. In fact, it is the high output impedance of LNTA that jointly with low impedance of the $\mathrm{N}$-path circuitry enables significant blocker attenuation at offset frequencies. In this case the demands for the input range (up to $0 \mathrm{dBm}$, i.e. 632 $\mathrm{mV}_{\mathrm{pp}}$ ), and respectively for the linearity and compression of the LNTA, are exacerbated since the attenuation is achieved at the output rather than at the input of the amplifier. Additionally, such an LNTA is challenged by the requirement of wideband (WB) operation typical of the contemporary multi-band radios. 


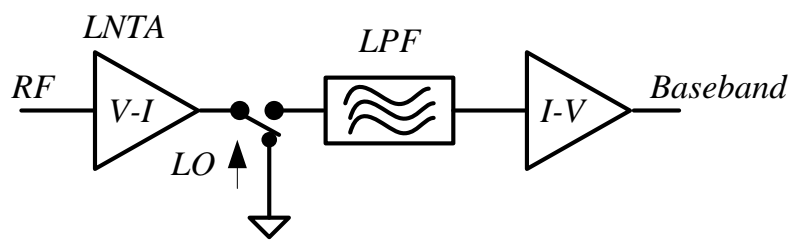

Figure 1.1: Conceptual diagram of the low-pass blocker filtering (current-mode receiver) in [11].

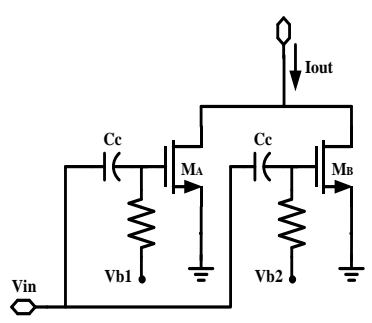

(a)

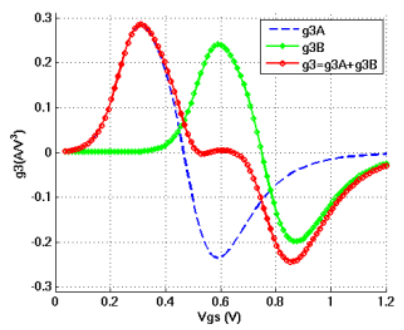

(b)

Figure 1.2: (a) DS technique with dual-NMOS. (b) Third-order distorion terms of the main transistor $\left(\mathrm{g}_{3 \mathrm{~A}}\right)$, auxiliary transistor $\left(\mathrm{g}_{3 \mathrm{~B}}\right)$, and total output $\left(\mathrm{g}_{3}\right)$ in [20].

The LNTA nonlinearity originates from two major sources: nonlinear transconductance which converts linear input voltage to nonlinear output drain current, and nonlinear output conductance, the effect of which is evident under large output voltage swing. The latter can be avoided using a low impedance output load that is usually achieved using a passive mixer followed by a transimpedance amplifier (TIA) [10-15].

Several techniques exist to improve linearity of LNAs [18]. The optimization of gate bias voltages can fairly improve linearity of LNA [19] but it leads to reduced range of the input amplitudes and increased sensitivity to process variation. The WB negative feedback by resistive source degeneration also improves linearity but limits the voltage headroom of the devices and adds extra noise. Superposition of an auxiliary transistor to cancel nonlinearity of the main device, called derivative superposition (DS), extends fairly the linear gain range $[20,21]$ as shown in Fig. 1.2. Its variant referred to as the complementary DS also improves the second order nonlinearity of the amplifier [22]. More recently, this technique has been also presented in $[24,26,16]$. Unlike DS, in the post-distortion technique (PD) the auxiliary device operates in saturation and is controlled by the output voltage. The PD advantage is in superior PVT robustness as demonstrated e.g. in [27]. 
Other critical concerns in LNA/LNTA design i.e., the input matching and noise figure (NF) usually cannot be compromised. A popular wideband matching technique exploits the common gate $(\mathrm{CG})$ circuit with its input impedance approximated by the inverse of the front device transconductance $\left(1 / \mathrm{g}_{\mathrm{m}}\right)$. Since in this case $\mathrm{g}_{\mathrm{m}}$ is virtually bound to 20 $\mathrm{mS}$, achieving larger effective values of the amplifier transconductance requires an extra amplification stage. To guarantee NF of the CG amplifier below $3 \mathrm{~dB}$ extra mechanisms are necessary, such as negative /positive feedback [32, 33], output noise cancellation using an auxiliary amplifier [29] (also called feed forward cancellation), or capacitive cross coupling when a balanced circuit is used [28] as shown in Fig. 1.3. Another WB matching technique providing a low NF is based on the reactive feedback which requires on-chip RF transformers [30].

A combination of a low noise figure with high linearity for wideband LNTA applications in CMOS was presented in [10-15, 22, 23, 34]. In particular, the noise cancelling receiver demonstrated in [13] extends the noise cancelling to the N-path filter / mixer resulting in the superior NF, but it consumes more power than the circuits using conventional noise cancellation [10-12, 14, 15].

The second work in Part I from receiver side is design for on-chip test. As the complexity of RF integrated circuits is increasing, the task of testing and debugging becomes more and more challenging. The degradation in performance or the drift from the optimal operation points may cause systems to fail. To prevent this effect and ensure quality in terms of process, voltage and temperature variations (PVT), test and calibration of the contemporary RF chips appear to be a must. This approach can be largely facilitated by on-chip techniques (DfT) and in the case of mass production the test cost can be cut as well.

Among different test circuits integrated on a chip, RF detectors play a vital role as signal level meters enabling various measurements like RF gain, compression or selectivity. A simple CMOS RF detector for on-chip amplitude measurements by RF to DC conversion within the band of $1-5 \mathrm{GHz}$ was proposed in [35]. In order to achieve a higher dynamic range, amplification before converting to DC was proposed in [36-38]. However, this technique is limited by the frequency bandwidth of the amplifier preceding the actual RF detector if a large gain value and consequently, a large dynamic range is a target. Another technique where MOS devices operate in sub-threshold region was proposed in [40]. This approach helps to minimize the effects of PTV variations and to enhance the detector bandwidth, but it is not well suited for larger input signals. For a detector with enhanced conversion gain the RF-DC transfer characteristic cannot cover the entire input range. To address this problem the sub-ranging technique using a digitally controlled bias network was proposed in [41, 42].

As process variations can influence characteristics of any circuit on a chip, a focused calibration of the RF detectors is necessary to ensure accuracy of the on-chip measurements. A replica detector was introduced to enable accuracy verification of detectors on a chip against the primary detector calibrated by external equipment [39]. 


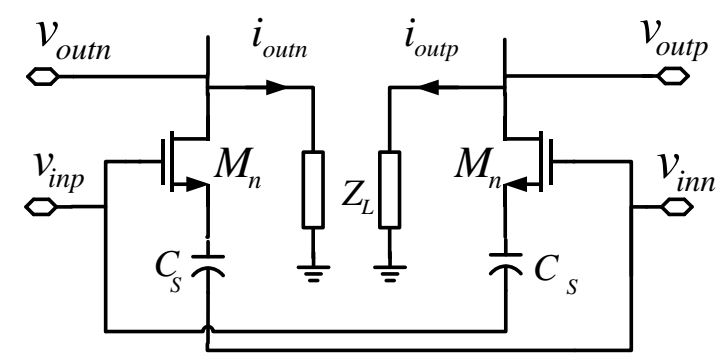

Figure 1.3: Capacitive cross-coupling technique in [28].

A calibration technique for all on-chip detectors, based on DC measurements and multivariate nonlinear regression was proposed in [43].

\subsection{Part II: High Speed SC DACs for wideband wireless transmitters}

The Part II of thesis is high speed SC DACs for wideband wireless transmitters. On transmitter side, on the pace towards broadband connectivity in wireless telecommunication systems increasingly more demands are placed on the performance and speed of the digital-to-analog converters (DACs). To enable ever higher data rates, wider channel bandwidths and advanced DSP techniques such as OFDM or multi-bit QAM are required that in terms of design, transform onto speed, dynamic range, and the linearity specifications. Implemented in CMOS technology DACs have proven to meet the broadband communication challenges in particular for the speed of CMOS devices and also for low manufacturing cost. The evolution of the unlicensed $60-\mathrm{GHz}$ radio band [57.2-65.8] GHz is a result of the increasing demand for high-data-rate shortrange wireless communication. This has motivated the development of recent standards, such as WiGig (IEEE 802.11ad) [44], ECMA-387 [45], and WirelessHD [46] as shown in Fig. 1.4. Fig. 1.5 shows the 60-GHz frequency band allocations in USA, Canada, Japan, Australia, Korea, China and Europe. The $60-\mathrm{GHz}$ band is divided into four channels which have a $1.76 \mathrm{GHz}$ RF channel bandwidth for each. One the fundamental part of wireless transmitters is digital-to-analog converter (DAC) which is required to have a bandwidth (BW) greater than $880 \mathrm{MHz}$ and a resolution greater than 6 bits in order to support those standards in terms of different modulation schemes [47-51]. 


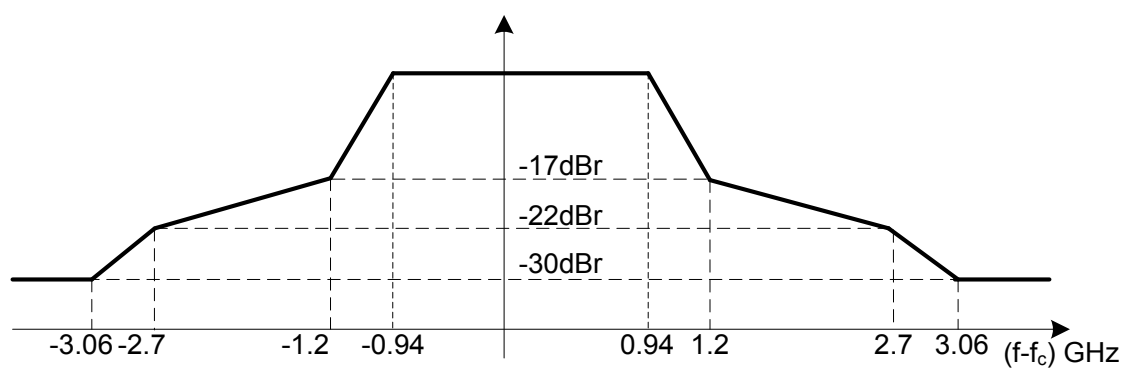

Figure 1.4: Spectral mask of WiGig for single channel operation in [44].
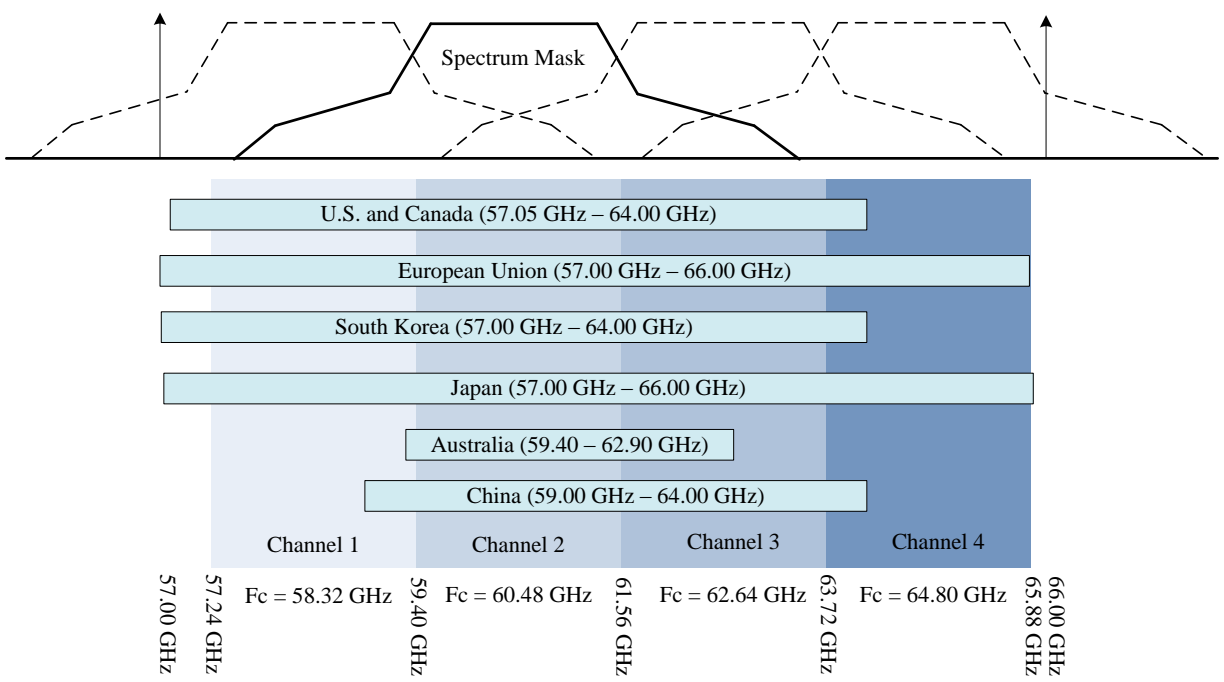

Figure 1.5: $60 \mathrm{GHz}$ Band Channel Plan and Frequency Allocation by Region in [44].

Recently the current-steering architectures [52-59] have been mainly focused on due to their simplicity and the achieved good performances among DACs. However the output impedances of current-cells cause the static non-linearity [52-55]. One solution for it is to use the elevated voltage supply for analog part in order to have more headroom which limits the supply voltage scalability of technology. Balancing the output impedance by using interleaved topology is another solution but it introduces other problems such as gain error and duty cycle error [59]. Another source of error which causes distortion in current-steering DACs is the mismatch of the saturation 
current of a MOS transistor [52-55, 60]. On the other hand, the capacitive DACs (SC DACs) have not drawn much attention except for few recent publications [61-63]. In fact, SC DACs have some advantages over the current-steering architectures [61, 62]. Specifically, lower capacitor mismatch helps SC array to achieve better linearity. Operating in triode region as a switch, transistor does not need voltage headroom from high supply. It therefore helps SC DAC array to show the supply-voltage scaling potential for more advanced technology. The power consumption is also low because its operation is based on charge redistribution.

\subsection{Thesis Organizations}

The rest of this thesis is organized as follows. Chapter $\mathbf{2}$ discusses about the design considerations of RF receiver front-ends including RF LNAs, design for on-chip test and high speed SC DACs for wideband wireless transceivers. The detailed design, analysis and implementation of RF receiver front-end and RF LNA are described in Chapter 3. Chapter 4 presents the design of RF detector for testability. The design and analysis of high speed capacitive pipeline DACs for wideband wireless applications are also presented in Chapter 5. Based on that analysis of pipeline DAC the 12-bit splitsegmented SC DAC is proposed, designed and implemented in 65-nm CMOS technology and provided in Chapter 6. To the authors' knowledge this DAC achieved the highest clock frequency (5 GS/s) and highest signal bandwidth $(1 \mathrm{GHz})$ among recent reported SC DACs. Furthermore it satisfies the spectral mask of the IEEE 802.11ad WiGig standard with a second-order reconstruction filter so it can be used for $60-\mathrm{GHz}$ radio baseband. Finally Chapter 7 concludes the thesis and sketches the future works. 


\section{Chapter 2}

\section{Design Considerations of RF Receiver Front-end, Testability and high speed SC DACs}

\subsection{Introduction}

As mentioned in Chapter 1, the thesis focuses to solve the key challenges of the wideband wireless transceiver blocks which consist of RF receiver front-end and design for on-chip test on receiver side and wideband SC DACs on transmitter side. This Chapter describes the design considerations of RF receivers including RF LNAs, design for test (DfT) modules and high speed capacitive DACs. 


\subsection{Design considerations of $\mathrm{RF}$ receivers}

Part I of thesis is wideband RF receiver front-end. The design considerations of RF receiver particularly sensitivity, noise figure (NF), selectivity, linearity and dynamic therefore will be discussed in this section.

\subsubsection{Sensitivity and noise figure}

Receiver sensitivity is defined as the ability to cope up the minimum signal level with the acceptable signal-to-noise ratio (SNR) which is defined by the modulation scheme of receivers. It is one of the key specifications of receivers and calculated in [114] as

$$
S_{\text {min }}=10 \log \left(P_{S, \min }\right)=10 \log \left(k T_{0}\right)+10 \log (B W)+N F_{R x}+S N R_{\min },
$$

where $10 \log \left(k T_{0}\right)=-174(\mathrm{dBm} / \mathrm{Hz})$ is thermal noise power density with $k=1.38 \times 10^{-20}$ $\mathrm{mW}$.sec $/{ }^{0} \mathrm{~K}$ is Boltzman constant and $T_{0}=290^{0} \mathrm{~K}, B W$ is the channel bandwidth, $N F_{R x}$ is the receiver noise figure, $S N R_{\text {min }}$ is determined by the modulation and demodulation scheme. Therefore sensitivity of receiver is strongly dependent on $N F_{R x}$.

\subsubsection{Selectivity}

Selectivity is another key specification of receivers and defined as the ability to reject all unwanted signals (blockers, interferers). RF/IF filters are still strong candidates for selectivity but additional blocks like amplifiers and mixers have selectivity as well. For the modern receivers, the technique of $N$-path filter [1-10] achieves good selectivity and rejection ratio comparable to $\mathrm{SAW}$-less filters.

\subsubsection{Nonlinearity and Intermodulation}

Active devices have both linear and non-linear operating regions. When the input/internal signal is large enough, it causes distortion harmonics which can interrupt or destroy the wanted signal. Fig. 2.1 shows one of example effects of nonlinearity on wanted signal [64]. The nonlinearity of receiver is mainly determined by its active components such as amplifiers, mixers, active filters. 


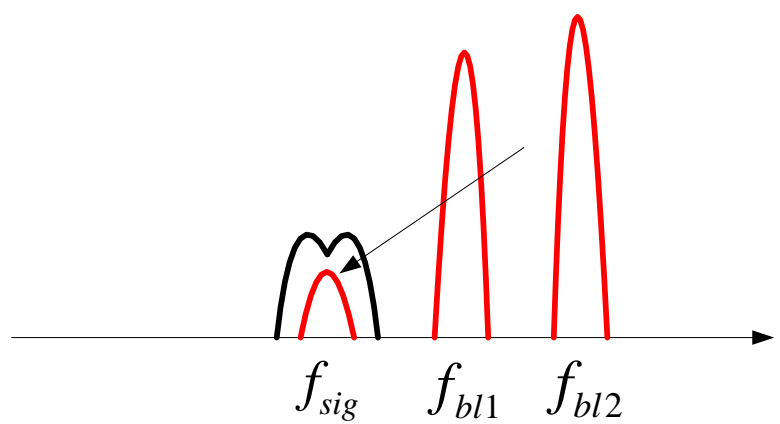

Figure 2.1: Effect of intermodulation from two blockers on wanted signal.

\subsubsection{Dynamic range}

Dynamic range is known as the ratio between the maximum input level to the minimum input level at which the system can tolerate and able to provides the reasonable signal quality. The lower end of the dynamic range is defined by the sensitivity of the receiver when its upper end is bounded by the maximum input level that the system can tolerate with acceptable distorting signal.

\subsection{Design considerations of RF LNAs}

As the first stage of RF receiver front-end, LNA plays an important role of NF and linearity of front-ends. The design considerations of RF LNAs including NF and linearity (IIP3, IIP2) therefore will be discussed. The sources of noise and non-linearity that significantly contribute to the output will be described.

\subsubsection{Noise figure (NF)}

As the first stage of receivers, LNAs play a critical role in the overall performance. The noise figure of LNA directly adds to that of the receiver. It therefore is necessary to indentify the noise sources of LNA for calculating NF.

\subsubsection{Device noise}

\subsection{Thermal noise of resistors}




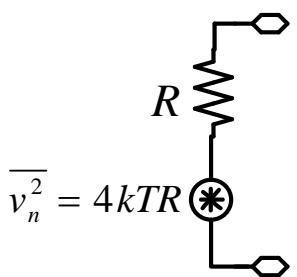

(a)

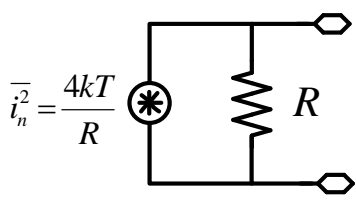

(b)

Figure 2.2: Thermal noise of resistor a) Thevenin b) Norton.

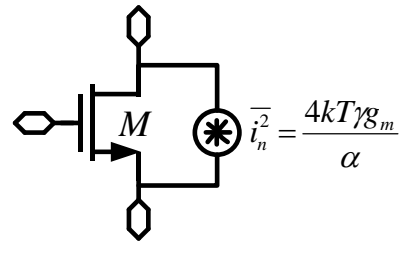

(a)

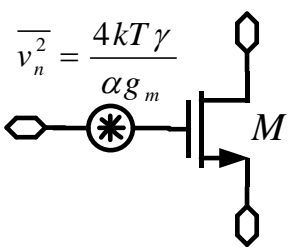

(b)

Figure 2.3: Noise models of MOSFET (a) current source (b) voltage source.

The noise model of resistors can be described using either Thevenin or Norton equivalent as shown in Fig. 2.2. The power spectrum density (PSD) of voltage and current is proportional to its value $R$ and inversion of $R$ respectively as

$$
\begin{gathered}
\overline{v_{n}^{2}}=4 k T R \\
\overline{i_{n}^{2}}=\frac{4 k T}{R}
\end{gathered}
$$

where $k$ is Boltzmann's constant, $T$ is the absolute temperature in Kelvin.

\subsection{Noise in MOSFETs}

When MOS transistors work in saturation region their thermal noise can be modelled either current or voltage source as shown in Fig. 2.3 as

$$
\begin{aligned}
& \overline{v_{n}^{2}}=\frac{4 k T \gamma}{\alpha g_{m}}, \\
& \overline{i_{n}^{2}}=\frac{4 k T \gamma g_{m}}{\alpha},
\end{aligned}
$$

where $\gamma$ is the excess channel thermal noise coefficient, and $\alpha=g_{m} / g_{d 0}$, with $g_{m}$ as the device transconductance and $g_{d 0}$ as zero-biased channel conductance. The noise coefficient is $2 / 3$ for long-channel transistors and higher for short-channel devices [64]. 
Another component of thermal noise which becomes increasingly more important as the gate length is scaled down is caused by the gate resistance of MOSFETs. According to [65], this resistance and its voltage noise are calculated as

$$
\begin{aligned}
& R_{G}=\frac{W}{L} R_{s h}, \\
& \overline{v_{n}^{2}}=\frac{4 k T R_{G}}{3},
\end{aligned}
$$

where $R_{s h}$ is the sheet resistance of the polysilicon gate with transistor width $W$ and length $L$. Typically this noise is much less than that of the channel.

Another noise which is dominant in MOS in low frequency is "flicker" or " $1 / \mathrm{f}$ " noise. Similarly the voltage and current noise can model as

$$
\begin{aligned}
& \overline{v_{n}^{2}}=\frac{K}{W L C_{o x}} \frac{1}{f}, \\
& \overline{i_{n}^{2}}=g_{m}^{2} \frac{K}{W L C_{o x}} \frac{1}{f},
\end{aligned}
$$

where $K$ is a process dependent constant. In CMOS technology, $K$ is lower for PMOS than for NMOS transistors because the former carry charge well below the silicon-oxide interface and hence suffer less from "surface states" [66].

The corner frequency is defined as the frequency where the flicker noise is equal to the thermal noise

$$
f_{c}=\frac{K}{W L C_{o x}} \frac{\alpha g_{m}}{4 k T \gamma} .
$$

It falls in the range of tens or even hundreds of megahertz in recent MOS technologies [64].

\subsubsection{Noise figure}

For electronic circuit and system, the signal-to-noise ratio (SNR) is defined as the signal power divided by the noise power. The noise factor/noise figure $(\mathrm{F} / \mathrm{NF})$ is also defined as

$$
N F=\frac{S N R_{\text {in }}}{S N R_{\text {out }}} .
$$

Noise figure (NF) can be expressed in decibels as 


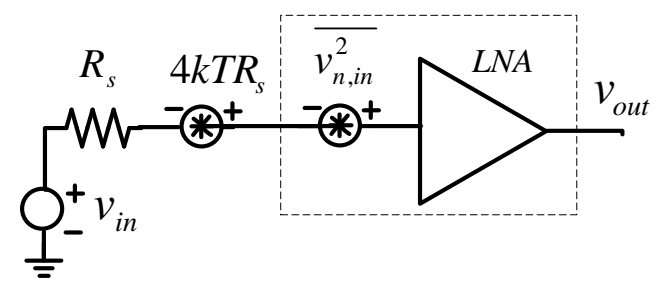

Figure 2.4: The input-referred noise voltage model of LNA.

$$
\left.N F\right|_{d B}=10 \log \left(\frac{S N R_{\text {in }}}{S N R_{\text {out }}}\right) .
$$

The input-referred noise model as shown in Fig. 2.4 is usually used to calculate NF for LNA. For example in this case NF will be equal to

$$
N F=1+\frac{\overline{v_{n, i n}^{2}}}{4 k T R_{s}} .
$$

For the cascaded stages, the total NF can be calculated as

$$
F_{\text {total }}=F_{1}+\frac{F_{2}-1}{G_{1}^{2}}+\frac{F_{3}-1}{G_{1}^{2} G_{2}^{2}}+\ldots
$$

where $G_{1}, G_{2}$ are voltage gains of the first and second stages of chain, $F_{\text {total }}, F_{1}, F_{2}, F_{3}$ are noise factors of total, first, second and third stages respectively.

\subsubsection{Gain}

The gain of LNA must be large enough to minimize the noise contribution of subsequent stages but it also makes the nonlinearity of the subsequent stages more pronounced. Therefore the choice of this gain leads to a compromise between the noise figure and linearity of receiver based on (2.15-2.16).

The Friis formulas for noise factor and IIP3 are respectively

$$
\begin{gathered}
F_{\text {receiver }}=F_{L N A}+\frac{F_{\text {mixer }}-1}{G_{L N A}^{2}}+\ldots, \\
\frac{1}{A_{I P 3, \text { receiver }}^{2}}=\frac{1}{A_{I P 3, L N A}^{2}}+\frac{G_{L N A}^{2}}{A_{I P 3, \text { mixer }}^{2}}+\ldots,
\end{gathered}
$$

where $G_{L N A}$ is a voltage gain of LNA, $F_{\text {receiver }}, F_{L N A}, F_{\text {mixer }}$ are noise factors of receiver, LNA and mixer respectively, $A_{I P 3, \text { receiver }}, A_{I P 3, L N A}, A_{I P 3, \text { mixer }}$ are third intercept point (IP3) amplitutes of receiver, LNA and mixer respectively. 


\subsubsection{Input return loss}

In order to avoid the reflected power, the input matching of LNA is required to be good enough. The input return loss is defined as the reflected power divided by the incident power as

$$
\Gamma=\left|\frac{Z_{\text {in }}-R_{s}}{Z_{\text {in }}+R_{s}}\right|^{2},
$$

where $Z_{i n}, R_{s}$ are the input impedance of LNA and source impedance respectively. Typically the input return loss less than $-10 \mathrm{~dB}$ over the working range of frequency is acceptable.

\subsubsection{Stability}

A parameter often used to characterize the stability of circuit is the "Stern stability factor," defined as

$$
K=\frac{1+|\Delta|^{2}-\left|S_{11}\right|^{2}-\left|S_{22}\right|^{2}}{2\left|S_{11}\right|\left|S_{22}\right|},
$$

where $\Delta=S_{11} S_{22}-S_{12} S_{21}$. If $K>1$ and $\Delta<1$ then the circuit is unconditionally stable. A high reverse isolation $\left(S_{12}\right)$ is also necessary for suppressing the LO leakage to the input of the LNA.

\subsubsection{Linearity}

\subsubsection{Harmonic distortion}

In order to model and calculate the harmonic distortion of a nonlinear system, the output of memoryless system is simplified as

$$
y(t) \approx \alpha_{1} x(t)+\alpha_{2} x^{2}(t)+\alpha_{3} x^{3}(t),
$$

where $x(t)=A \cos (\omega t)$ is the input sinusoidal signal. It can be expanded as

$$
y(t) \approx \frac{\alpha_{2} A^{2}}{2}+\left(\alpha_{1} A+\frac{3 \alpha_{3} A^{3}}{4}\right) \cos (\omega t)+\frac{\alpha_{2} A^{2}}{2} \cos (2 \omega t)+\frac{\alpha_{3} A^{3^{3}}}{4} \cos (3 \omega t) .
$$

The first term on the right-hand side in (2.20) is a DC quantity caused by second-order nonlinearity while the second, third and fourth terms are the fundamental, second and third harmonics respectively. 


\subsubsection{Gain compression}

The fundamental gain in (2.20) is $\left(\alpha_{1} \mathrm{~A}+3 \alpha_{3} \mathrm{~A}^{3} / 4\right)$. Typically in CMOS technology, $\alpha_{1} \alpha_{3}$ $<0$ leads to the compressive behaviors when the input signal is large enough. To quantify this effect the definition of " $1-\mathrm{dB}$ compression point" is introduced. It is the input signal level that causes the gain to drop by $1 \mathrm{~dB}$ and approximated as

$$
A_{i n, 1 d B}=\sqrt{0.145\left|\frac{\alpha_{1}}{\alpha_{3}}\right|} .
$$

The $1-\mathrm{dB}$ compression point represents a $10 \%$ reduction in the gain and is widely used to characterize RF circuits and systems.

\subsubsection{Cross modulation}

When a large interferer accompanies the received signal the compression caused another adverse effect. This phenomenon lowers the signal-to-noise ratio (SNR) at the receiver output and is called "desensitization". In order to quantify this, the signal which consists of the desired component and the interferer is applied to the input of system as

$$
x(t)=A_{1} \cos \left(\omega_{1} t\right)+A_{2} \cos \left(\omega_{2} t\right),
$$

where $A_{1}, A_{2}$ are the amplitude of signal and interferer respectively hence $A_{1} \ll A_{2}$. The fundamental component of the output can be simplified as

$$
y_{1}(t)=\left(\alpha_{1}+\frac{3 \alpha_{3} A_{2}^{2}}{2}\right) A_{1} \cos \left(\omega_{1} t\right)
$$

The fundamental signal gain in (2.23) is reduced due to $A_{2}$ if $\alpha_{1} \alpha_{3}<0$. The signal is "blocked" when it drops to zero for sufficiently large interferer.

Moreover variations in $A_{2}$ affect the amplitude of the signal at $\omega_{1}$ and cause "cross modulation". For example assuming the interferer is an amplitude-modulated signal as

$$
A_{2}\left(1+m \cos \left(\omega_{m} t\right)\right) \cos \left(\omega_{2} t\right)
$$

where $m$ is a constant and $\omega_{m}$ is the modulating frequency, the fundamental term of output will be as

$$
y_{1}(t)=\left[\alpha_{1}+\frac{3}{2} \alpha_{3} A_{2}^{2}\left(1+\frac{m^{2}}{2}+\frac{m^{2}}{2} \cos \left(2 \omega_{m} t\right)+2 m \cos \left(\omega_{m} t\right)\right)\right] A_{1} \cos \left(\omega_{1} t\right) .
$$

From (2.25) it can be seen that the desired signal at the output suffers from amplitude modulation at $\omega_{m}$ and $2 \omega_{m}$. 


\subsubsection{Intermodulation}

The intermodulation (IM) is caused by mixing (multiplication) of two interferers which accompany the desired signal. Similarly the effect can be evaluated when applying two interferers at $\omega_{1}$ and $\omega_{2}$ to a nonlinear system. Among the harmonic components which are generated at the output, the third-order IM products at $2 \omega_{1}-\omega_{2}$ and $2 \omega_{2}-\omega_{1}$ are of particular interest since they can fall onto the desired channel and corrupting the signal .Their amplitudes are $\left(3 \alpha_{3} A_{1}^{2} A_{2} / 4\right)$ and $\left(3 \alpha_{3} A_{2}^{2} A_{1} / 4\right)$.

\subsubsection{Third intercept point}

To characterize IM two pure sinusoids of equal amplitudes called "two-tone test" are applied to the input. The amplitude of the output IM products is then normalized to that of the fundamentals and is calculated as

$$
I M=20 \log \left(\frac{3 \alpha_{3} A^{2}}{4 \alpha_{1}}\right),
$$

where $A$ is the peak amplitude of each tone and IM has unit of $\mathrm{dBc}$ meaning decibels with respect to the carrier to emphasize the normalization.

If the amplitude of each tone rises, that of the output IM products increases more sharply. The third-order intercept point is defined when the amplitude of the IM products equal to that of fundamental tones at the output. The input/output third intercept point (IIP3/OIP3) is the input/output level when IP3 occurs. The amplitude of IIP3 can be calculated as

$$
A_{I I P 3}=\sqrt{\mid \frac{4 \alpha_{1}}{3 \alpha_{3} \mid}} .
$$

From (2.21) and (2.27), the IIP3 is around $9.6 \mathrm{~dB}$ greater than 1-dB compression point $\left(\mathrm{P}_{1-\mathrm{dB}}\right)$. The IIP3 $(\mathrm{dBm})$ can also be calculated based on the input/ouput power of each tone $\left(\mathrm{P}_{\text {in }} / \mathrm{P}_{\text {out }}\right)$ and output third-order modulation (IM3 $\left.3_{\text {out }}\right)$

$$
\left.I I P 3\right|_{d B m}=\frac{\left.\Delta P\right|_{d B m}}{2}+\left.P_{i n}\right|_{d B m} .
$$

where $\Delta P=P_{\text {out }}-I M 3_{\text {out }}$.

For the cascaded stages, the total IIP3 can be calculated as

$$
\frac{1}{A_{I P 3, \text { total }}^{2}}=\frac{1}{A_{I P 3,1}^{2}}+\frac{G_{1}^{2}}{A_{I P 3,2}^{2}}+\frac{G_{1}^{2} G_{2}^{2}}{A_{I P 3,3}^{2}}+\ldots,
$$

where $G_{1}, G_{2}$ are voltage gains of the first and second stages of chain, $A_{I P 3, t o t a l}, A_{I P 3,1}$, $A_{I P 3,2}, A_{I P 3,3}$ are third intercept point (IP3) amplitutes of total, first, second and third stages respectively. 
The linearity of the LNA is critical in wideband (WB) receiver where may have a large number of strong interferers. The ultra-wideband (UWB), software-defined, and cognitive radios are examples of $\mathrm{WB}$ receivers.

\subsubsection{Bandwidth}

Typically the LNA must provide a relatively flat response for the frequency range of interest. Preferably variation of gain should be less than $1 \mathrm{~dB}$ therefore the $\mathrm{BW}_{-3 \mathrm{~dB}}$ of LNA must be substantially greater than the actual band. The fractional BW is defined as the total $\mathrm{BW}_{-3 \mathrm{~dB}}$ divided by the center frequency of the band.

\subsubsection{Power dissipation}

A direct trade-off among noise, linearity, and power dissipation is exhibited in LNA. Hower its NF is more critical than power since the dissipation of other blocks in receivers is more dominant.

\subsubsection{Input matching}

To avoid the reflected power at the input of LNA, it is necessary to have the input resistance of $50 \Omega$. This requirement limits the choice of LNA topologies for wideband design unless we sacrifice NF by adding 50- $\Omega$ resistor at input of LNA. Typically the input return loss $\left(\mathrm{S}_{11}\right)$ from (2.17) in $\mathrm{dB}$ should be less than $-10 \mathrm{~dB}$.

\subsubsection{Reverse isolation}

The reverse isolation is defined as $-S_{12}$ where $S_{12}$ is the reverse gain of LNA. The feedthrough from LO through mixer can reach to antenna if the reverse isolation of LNA is not good enough. Typically a value greater than $25 \mathrm{~dB}$ of isolation should be sufficient. Futhermore, it is necessary to have good reverse isolation in order not to affect the input matching and the intrinsic stability of the amplifier.

\subsection{The considerations of design for on-chip test}

The second work of the Part I is design for test (DfT). Several typical design considerations of RF detectors will be described.

\subsubsection{Input impedance of RF detectors}

In order not to affect on circuit under test (CUT), the input impedance of RF detector should be high enough over the operating band of frequency. Its minimum value should be 10 times greater than the impedance at the node of CUT it is connected. 
2.5 Design considerations of high speed capacitive DACs for wideband wireless

transmitters

\subsubsection{Area of on-chip test system}

The silicon area is another consideration in the mass production. DfT cannot be used if it does not have area less than $10 \%$ of the total area of CUT system.

\subsubsection{Wide bandwidth of RF detectors}

Definitely bandwidth of RF detector has to be wider than the bandwidth of CUT for ensuring the accuracy of measurement.

\subsubsection{Power dissipation of on-chip test system}

For mass production if it has DfT, the power constraint of DfT needs to be considered. However if DfT is only used in the phase of research and development, it does not matter since it is tested in certain time for debugging problem of CUT.

\subsubsection{Gain and dynamic range of RF detectors}

Depending on CUT and its applications, how large gain is required from RF detector. But in most cases the detected signal needs to be amplified and needs gain. On the othe hand, the dynamic range (detectable range) is also important and needs to be considered.

\subsection{Design considerations of high speed capacitive DACs for wideband wireless transmitters}

Part II of thesis is design of high speed SC DACs. So the design considerations of its will be discussed. For high speed applications, the performance of high resolution, capacitive DACs appears to be limited by switching noise, clock feedthrough, settling time, kT/C noise and capacitor mismatch depending upon the DAC architecture and SC array topology. The output driver significantly causes distortion for the output signal in some applications which need high output swing such as wireline internet.

\subsubsection{The $\mathrm{kT} / \mathrm{C}$ nosie}

The kT/C noise in track and hold circuit in [62] is critical since capacitance must be small for fast switching. However, its effect is negligible for the 12-bit resolution and high speed DACs in $[63,67]$. The lower bound of unit capacitance can be found for the pipeline SC array as

$$
C_{u}>\frac{4.67 k T\left(2^{n}-1\right)^{2}}{V_{F S}^{2}},
$$

where $V_{F S}$ is the full-scale voltage, $n$ is the number of bits, $T$ is the absolute temperature in Kelvin, and $k \approx 1.38 .10^{-23}(\mathrm{~J} / \mathrm{K})$ is the Boltzmann's constant. 


\subsubsection{Capacitor mismatch}

As capacitor has good matching feature, the performance of capacitive DAC is not limited by capacitor mismatch [67, 61-63]. Another lower bound of unit capacitance can be simplified for the SC DAC array as

$$
C_{u}>9 K_{C} K_{\sigma}^{2}\left(2^{n}-1\right)
$$

where $K_{\sigma}$ (matching coefficient) and $K_{C}$ (capacitance density) are technology constants provided by the chip manufacturers, $n$ is the number of bits.

\subsubsection{Settling time}

Definitely settling time has to be considered for high speed design. However, it shows feasility to design SC DAC with 12-bit resolution and speed greater than $1 \mathrm{GS} / \mathrm{s}$ in [63, 67]. The lower bound of the off-resistance of switch and the upper bound of the onresistance of switch are found in pipeline SC array respectively as

$$
\begin{gathered}
R_{\text {off }}>-\frac{t_{\text {pulse }}}{2 C_{u}} \frac{1}{\ln \left(1-2^{-n-1}\right)}, \\
R_{\text {on }}<\frac{t_{\text {pulse }}}{2 C_{u}} \frac{1}{\ln \left(2^{n+1}\right)},
\end{gathered}
$$

where $t_{\text {pulse }}$ is the pulse width of clock.

\subsubsection{Clock feed-through effect}

For clock frequency greater than $1 \mathrm{GS} / \mathrm{s}$, the clock feed-through causes charged/discharged voltage errors which result in distortion at the output of pipeline SC array [63]. Another lower bound of unit capacitance due to clock feed-through can be found for it as

$$
C_{u}>\left(K_{d i f} K_{c o m p} 2^{n+1} \frac{V_{d d}}{V_{r e f}}-1\right) C_{P}(W),
$$

where $C_{p}(W)$ is parasitic capacitance of a switch with respect to the switch size, while $K_{\text {comp }}$ is a constant representing the feed-through compensation by the dummy devices. Assuming that the total mismatch between two single-ended branches of differential topology (including mismatch of switches, caps, jitter, skew) is $K_{d i f}$.

Nevertheless, this problem can be solved with split-segmented SC array architecture [67]. 


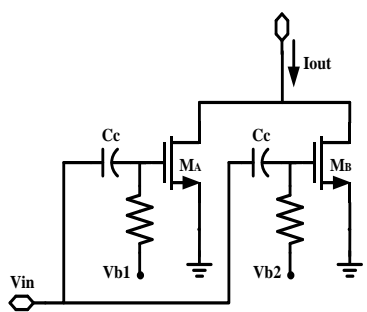

(a)

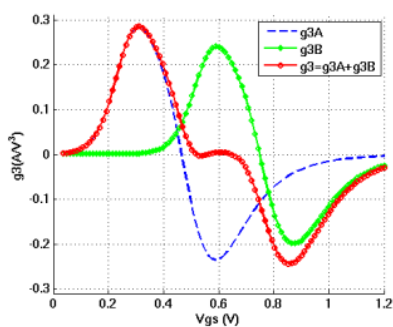

(b)

Figure 2.5: (a) Conventional DS technique. (b) Third-order distorion terms of the main transistor (g3A), auxiliary transistor (g3B), and total output (g3).

\subsubsection{Switching noise}

With resolution more than 10 bits and clock frequency greater than $2 \mathrm{GS} / \mathrm{s}$, simultaneous switching noise (SSN) significantly causes ripple/noise on supply which degrades much performance of SC DACs. It is considered as the most critical factor limiting performance of DACs [67]. However, there are several techniques to reduce it such as separating supplies, using more decaps, minimizing wire-bonding parasitic inductance and using dampling resistor. The lower bound of switch driver size due to SSN can be estimated as

$$
\left(\frac{W}{L}\right)_{n}<\frac{2 t_{r} V_{F S}}{\mu_{n} C_{o x}\left(\frac{V_{D D}}{2}-V_{t h}\right)\left(m L_{S S} V_{D D} 2^{n+1}-1.3 m L_{S S} V_{F S}\right)},
$$

where $m$ is number of drivers. The transistor sizes of switches in the switch-driver can be chosen depending on the rise time of clock $t_{r}$, supply $\left(V_{D D}\right)$, the output swing $\left(V_{F S}\right)$, the DAC resolution $(n)$, parasitic inductance of bonding-wires $\left(L_{S S}\right)$ and technology $\left(\mu_{\mathrm{n}} C_{o x}\right)$.

\subsubsection{Output driver}

For high output swing, the output driver has the main contribution of distortion for DACs [62]. The pre-distortion technique of calibration was applied to improve linearity of design. However, it can be eliminated without any form of calibration by using circuit technique such as derivative superposition (DS) shown in Fig. 2.5 and applied for wireless applications which need low output swing. 


\section{Part I}

\section{Wideband RF Receiver Front-end and Design for On-chip Test on Receiver Side}




\section{Chapter 3}

\section{Design of RF Receiver Front-end}

\subsection{Introduction}

The first work in Part I is design of two-stage highly selective receiver front-end. It is designed and implemented in $65 \mathrm{~nm}$ CMOS technology. Author's contributions from this work are design and analysis of LNAs and collaborating to design and implement the whole receiver front-end.

This Chapter presents a tunable receiver front-end with high blocker rejection achieved by two-stage impedance transformation. The front-end design is based on a low noise transconductance amplifier (LNTA) [115] which is well suited to reject outof-band blockers due to high ratio between its output impedance and on-resistance of switches used in the impedance transformation circuit.

The analysis and design of LNTA suitable for current-mode wideband front-end with RF N-path filtering in 0.5 to $3 \mathrm{GHz}$ frequency range will be also presented in this Chapter. The LNTA design combines two linearization techniques, namely the derivative superposition and resistive feedback, with NF reduction by double capacitive cross-coupling which results in superior noise performance. The resistive feedback also helps to attain good input matching without sacrificing gain of the common gate input 
Design of RF Receiver Front-end

stage. By using elevated supply voltage the LNTA can tolerate blockers up to $0 \mathrm{dBm}$ without compression. The mathematical analyses of NF and IIP3 are described in detail and the achieved estimates are verified by SpectreRF® simulation. The LNTA is implemented and measured in a two-stage highly selective receiver front-end, integrated using $65 \mathrm{~nm}$ CMOS technology [16].

\subsection{Design of Two-Stage Receiver Front-end}

To tolerate large blockers the rejection provided by one impedance transformation filter is usually insufficient. One solution to this problem is an extra $N$-path circuit embedded into the LNA [10]. However, in this case the rejection cannot be improved much because of loading effects in the LNA circuit.

To meet rejection typical of SAW filters we propose a two-stage architecture where separation between the stages is achieved by a high input impedance buffer incorporated in the second LNTA (Fig. 3.1). The circuit is differential and is designed in $65 \mathrm{~nm}$ CMOS with elevated supply voltage in the first stage to avoid compression by blockers [68]. The first LNA uses two linearization techniques, namely the derivative superposition and resistive feedback, with NF reduction by double capacitive crosscoupling which results in good noise performance [115] shown in Fig. 3.7. It will be discussed in detail in Section 3.3, 3.4 and 3.5. Still, 1.2 V devices have been used to take advantage of their lower threshold voltage compared to $2.5 \mathrm{~V}$ devices available in this technology as well. Since voltage stress could result in low reliability or damage of the devices, in this case, all bias voltages were applied to LNTA1 through an off-chip high-RC time constant circuit, shown in Fig. 3.2. To prevent loading for the first stage, which could degrade the filter transfer function, a simple CMOS buffer is added in front of LNTA2 as shown in Fig. 3.3. The schematic topology of LNTA2 is similar to LNTA1 except for the values of bias voltages, resistances and sizes of transistors. In this architecture the second stage also serves IQ down-conversion while the first stage helps to relax the requirements placed on linearity (IIP2) of the down-converter and on phase noise of the clock. It should be noted that an extra attenuation of the blocker by $\beta \mathrm{dB}$ results in reduction of the reciprocal mixing noise by $\beta \mathrm{dB}$ and of the intermodulation product (IM2) by $2 \beta \mathrm{dB}$. 


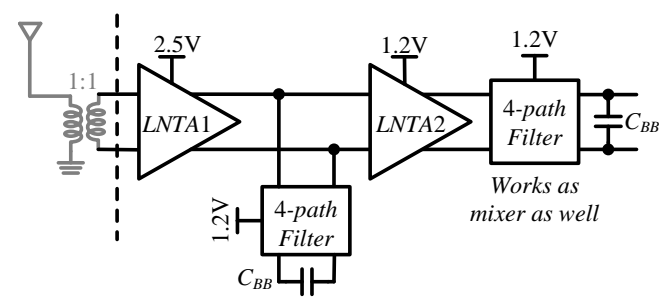

Figure 3.1: Architecture of selective two-stage RF front-end [16].

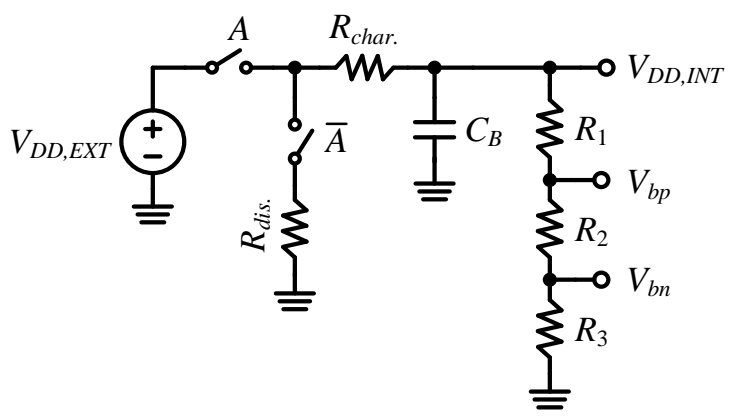

Figure 3.2: Startup circuitry for LNTA1 [69].

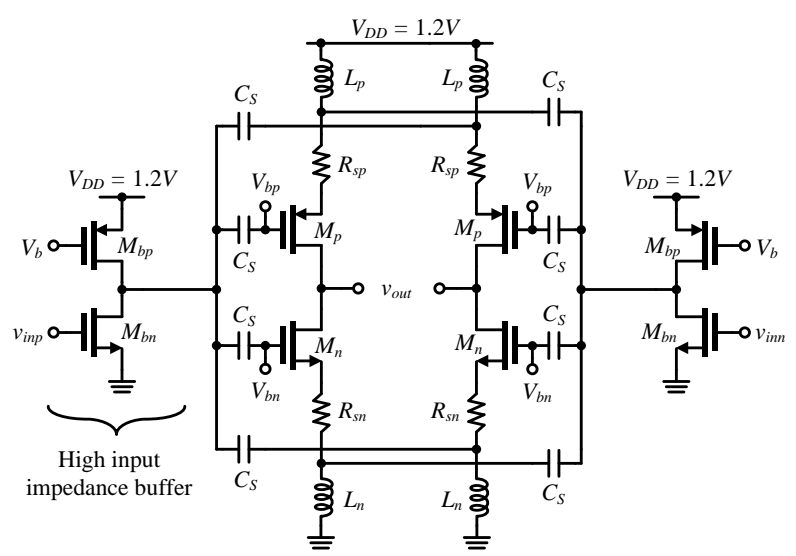

Figure 3.3: Circuit schematic of LNTA2 [69].

Importantly, each LNTA stage provides attenuation rather than gain for out-of-band blockers. Hence, the blockers can be well tolerated by the receiver chain and the out-of- 


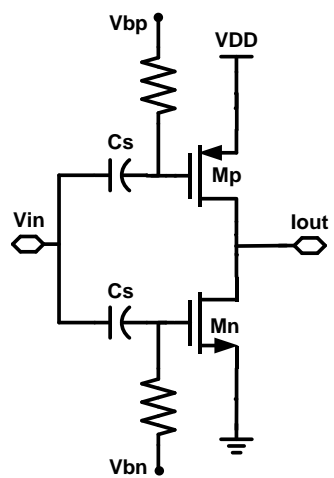

a)

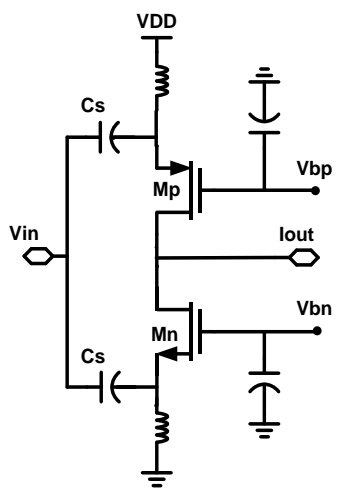

b)

Figure 3.4: LNTA complementary DS architectures, a) common source b) common gate.

band linearity (IIP2/IIP3) of the receiver is largely improved. Also the demands for the baseband filter and the dynamic range of the following $\mathrm{A} / \mathrm{D}$ converter are mitigated. It can be shown that the IIP3 of the two stage filter is

$$
1 / P_{I I P 3}=1 / P_{I I P 31}+G_{B 1} \eta_{1} / P_{I I P 32}
$$

From (3.1) we see how the blocker gain $G_{B 1}<1$ and rejection $\eta_{1}<1$, both of the first stage, allow to eliminate the IP3 contribution of the second stage.

\subsection{LNA Design}

Based on our preliminary work [26], here, we describe the LNTA design in detail, including a complete noise and linearity analysis.

For high linearity we refer to the complementary DS technique, which due to the reusing of current, gives also significant power savings. The complementary common gate (CG) architecture has been preferred over its counterpart, common source (CS) as shown in Fig. 3.4, for the ease in achieving wideband input matching and low noise figure.

By using appropriate bias voltages the nonlinear third order $g_{m}$ terms can be cancelled providing a high value of IIP3 [22-25]. In this case the pMOS is an auxiliary transistor with $g_{m}$ much smaller than that of nMOS. Large off-chip inductors $L_{1}, L_{2}$ rather than resistors are used to guarantee maximum bias voltage $V_{d s}$ and thereby to reduce the $g_{d s}$ nonlinearity that is increasingly pronounced in deep submicron CMOS. 


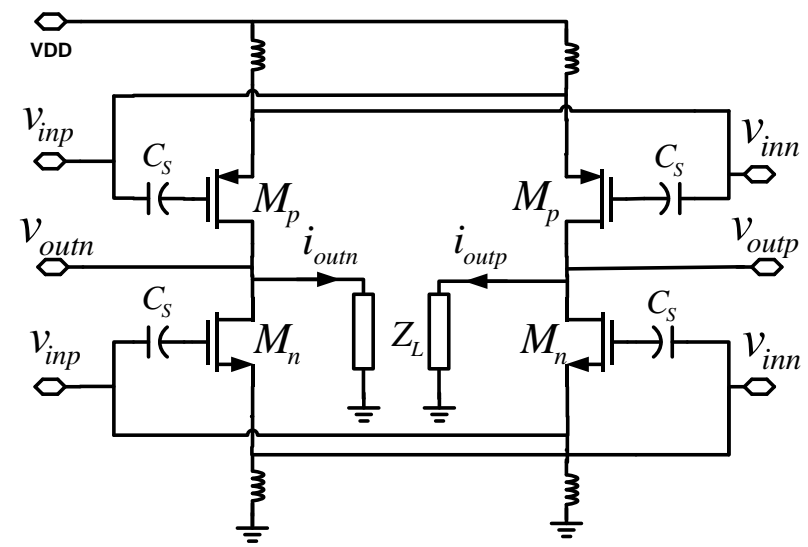

Figure 3.5: Differential LNTA implementing DS and capacitive cross-coupling technique (simplified schematic).

The input impedance and noise factor for the DS-CG circuit can be estimated from

$$
\begin{aligned}
& Z_{\text {in }}=\frac{1}{g_{m n}+g_{m p}} \\
& F \cong 1+\frac{\gamma}{\alpha\left(g_{m n}+g_{m p}\right) R_{s o}}
\end{aligned}
$$

where $R_{s o}$ is the source resistance, $\gamma$ is the excess channel thermal noise coefficient, and $\alpha=g_{m} / g_{d 0}$, with $g_{m}$ as the device transconductance and $g_{d 0}$ as zero-biased channel conductance.

Clearly, for perfect matching we have $F \approx 1+\gamma / \alpha$. In deep submicron CMOS $\gamma / \alpha>$ $2 / 3$, and to reduce its effect on $F$ we use a differential (balanced) variant of this circuit where the capacitive cross-coupling technique is adopted [28, 31]. In this case, $F$ can be estimated from

$$
F \cong 1+\frac{\gamma}{2 \alpha}
$$

according to partial noise cancellation achieved in this circuit. We observe that for $\gamma / \alpha$ $\approx 1$, the expected noise figure is $N F=10 \log (1.5) \approx 1.75 \mathrm{~dB}$.

Further noise factor improvement as we proposed in [26] can be achieved by using double capacitive cross-coupling circuit shown in Fig. 3.5 (to be discussed in detail in Sec. 3.4).

By sizing up the transistors the LNTA transconductance can be increased to some extent, but the input impedance is decreased accordingly and the reflection coefficient S11 is largely deteriorated. One solution to mitigate this tradeoff is based on the source degeneration technique. Acting as a local negative feedback it additionally improves 


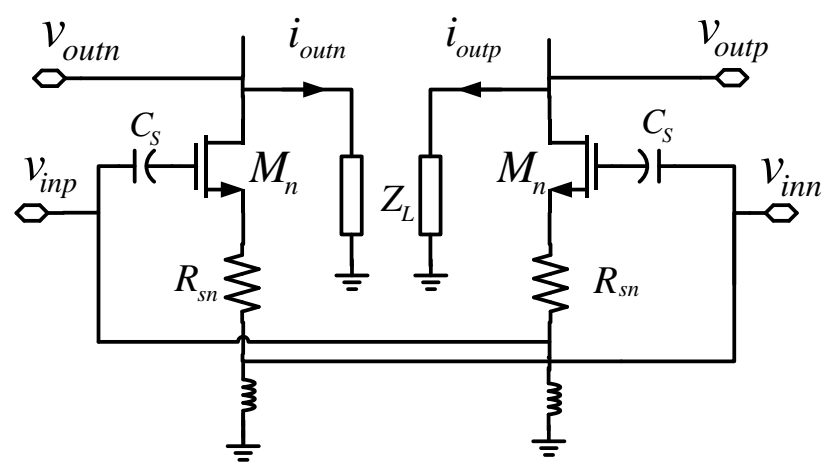

Figure 3.6: S11 and linearity improvement by resistive source degeneration.

circuit linearity. With a resistance $R_{s n}$ as shown in Fig. 3.6, the LNTA input impedance can be restored as demonstrated by (3.5) for the n-MOS part of the circuit. Knowing that $g_{m} / C_{g s}=2 \pi f_{T}$ where $f_{T} \approx 100 \mathrm{GHz}$, for simplicity we can assume $\omega C_{g s} / g_{m}=f / f_{T} \approx 0$. Then for the nMOS part of the circuit we find

$$
Z_{i n}^{(n)} \cong \frac{1+Z_{L} Y_{d s n}+R_{s n}\left(g_{m n}+Y_{d s n}\right)}{g_{m n}+Y_{d s n} / 2}
$$

where $Y_{d s n}$ is the drain-source admittance and $Z_{L}$ is the loading impedance while the inductor reactance goes to infinity. A similar formula can be derived for the pMOS part $\left(Z_{i n}^{(p)}\right)$ and assuming the drain-source admitances are small enough we find the LNTA input impedance as $Z_{i n}^{(p)} \| Z_{i n}^{(n)}$. The LNTA transconductance is inversely proportional to $Z_{\text {in }}$ that is

$$
G_{m}=\frac{1}{Z_{i n}^{(p)}(\omega)}+\frac{1}{Z_{i n}^{(n)}(\omega)}
$$

Hence, there is a tradeoff between the input matching and LNTA gain. For perfect matching no increase in $G_{m}$ is achieved. In practice, however, the requirement is $\mathrm{S} 11<-$ $10 \mathrm{~dB}$. To meet this condition the corresponding boundaries of $Z_{\text {in }}$ can be found: $Z_{i n} \in(0.67,2) R_{s o}$, where $R_{s o}$ is the matching resistance. In an extreme case, when $Z_{i n}=2 R_{s o}$ and $R_{s}=0$ we have $G_{m} \approx 2 / 2 R_{s o}$. Next, the transistors are sized up and by using $R_{s}$ we obtain $Z_{i n}=0.67 R_{s o}$ with the corresponding $G_{m} \approx 3 / R_{s o}$. This means $3 \times$ increase in $G_{m}(9.5 \mathrm{~dB})$ is feasible while $\mathrm{S} 11=-10 \mathrm{~dB}$. Clearly, larger values of $R_{s}$ should be avoided here to preserve a sufficient $V_{d s}$ voltage headroom. Also the noise factor is traded for $\mathrm{S} 11$ as the $R_{s}$ resistors add noise. Moreover, when the loading impedance $Z_{L}$ is selective (as for $\mathrm{N}$-path filters), its impedance goes down at offset 


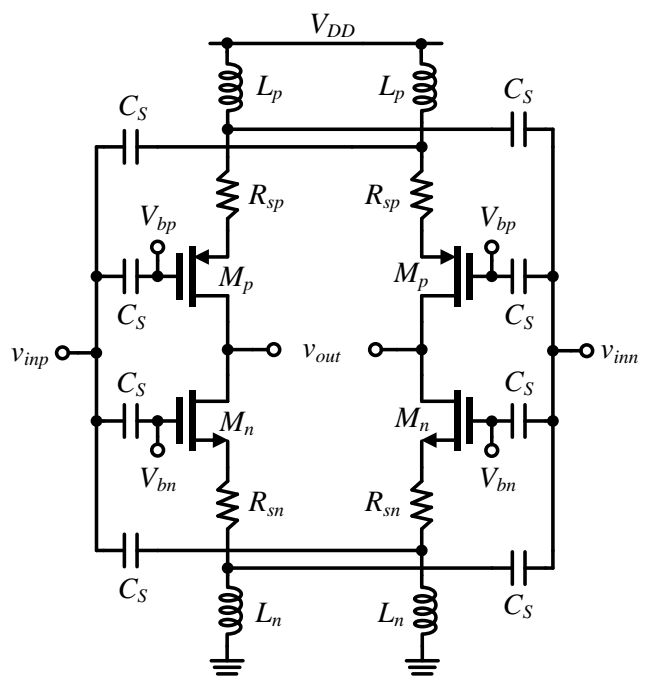

Figure 3.7: Circuit schematic of proposed wideband LNTA.

frequencies and the input impedance (3.5) is reduced accordingly providing thereby attenuation of blockers at the amplifier input.

The proposed final LNTA circuit, designed in $65 \mathrm{~nm}$ CMOS is shown in Fig. 3.7. It combines the discussed above techniques to achieve high linearity and a low noise figure over a wide frequency range. Four off-chip inductors providing reactance of a few hundred Ohms each are large enough to guarantee S11 $<-10 \mathrm{~dB}$ also at lower frequencies. Similarly, the coupling capacitances $C_{s}>10 \mathrm{pF}$ should be chosen $\left(X_{s}<2 \Omega\right)$ to avoid reduction of LNTA transconductance gain. Four of them (connected to transistor gates) must be integrated at the expense of the silicon area overhead. After choosing the bias voltages (to be discussed in Sec. 3.5) and the output DC equal to VDD/2 the sizes of the MOS transistors Mp, Mn were chosen to achieve the best thirdorder $g_{m}$ cancellation with $29 \mu \mathrm{m} / 65 \mathrm{~nm}$ and $48 \mu \mathrm{m} / 65 \mathrm{~nm}$, respectively. The source degeneration resistors providing correction of $\mathrm{S} 11$ are $R_{s p}=17 \Omega$ and $R_{s n}=111 \Omega$.

\subsection{LNA Noise Analysis}

The circuit model for noise analysis is shown in Fig. 3.8. In each half of the circuit there are five noise sources to be considered: $v_{n s}$ (source noise), $v_{n M 1}$ (of M1), $v_{n M 3}$ (of M3), $v_{n R s p}$ (of $R_{s p}$ ) and $v_{n R s n}\left(\right.$ of $R_{s n}$ ), using the following equations

$$
v_{n s}^{2}=4 k T R_{s o}, v_{n M 1}^{2}=\frac{4 k T \gamma_{1}}{\alpha_{1} g_{m 1}}, v_{n M 3}^{2}=\frac{4 k T \gamma_{3}}{\alpha_{3} g_{m 3}},
$$




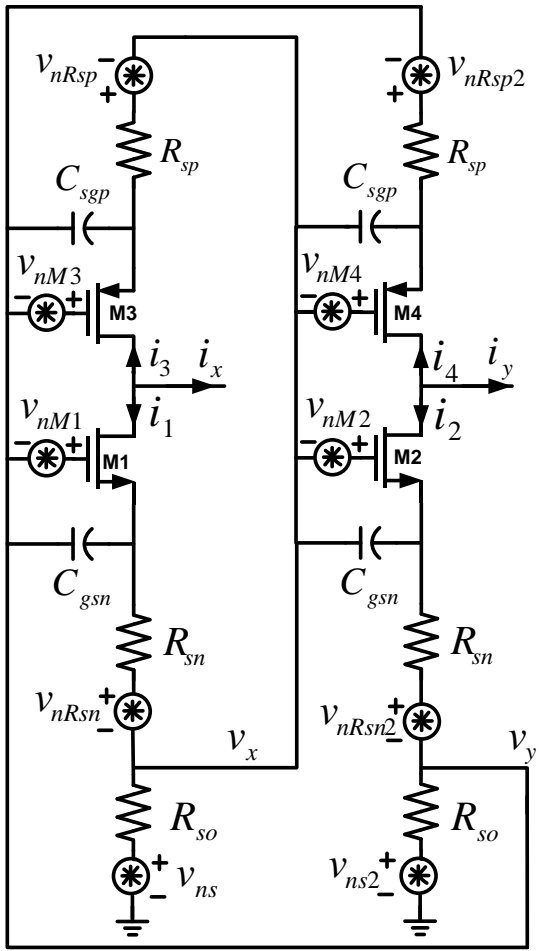

Figure 3.8: LNTA circuit for noise analysis.

$$
v_{n R s n}^{2}=4 k T R_{s n}, v_{n R s p}^{2}=4 k T R_{s p},
$$

where $k$ is Boltzmann's constant, $T$ is the absolute temperature in Kelvin. The differential noise current at the output $i_{n_{-} \text {out }}=i_{y}-i_{x}$ can calculated using superposition principle. In particular for $v_{n s}$ the currents $i_{1}, \ldots i_{4}$ as shown in Fig. 3.8 can be found as

$$
i_{1,3}=\left(v_{y}-v_{x}\right) g_{m 1,3 t}, i_{2,4}=\left(v_{x}-v_{y}\right) g_{m 2,4 t}
$$

with

$$
g_{m 1,2 t}=\frac{1}{R_{s n}\left(1+\frac{s C_{g s n}}{g_{m 1,2}}\right)+\frac{1}{g_{m 1,2}}}, g_{m 3,4 t}=\frac{1}{R_{s p}\left(1+\frac{s C_{s g p}}{g_{m 3,4}}\right)+\frac{1}{g_{m 3,4}}}
$$

Using Kirchhoff's Voltage Law (KVL) for the loop from $v_{x}$ to $v_{y}$ through $v_{n s}$ and Kirchhoff's Current Law (KCL) at nodes $v_{x}, v_{y}$ we have 


$$
\begin{aligned}
& v_{x}-v_{y}=v_{n s}+R_{s o}\left[i_{1}\left(1+\frac{2 s C_{g s n}}{g_{m 1}}\right)+i_{3}\left(1+\frac{2 s C_{s g p}}{g_{m 3}}\right)\right] \\
& -R_{s o}\left[i_{2}\left(1+\frac{2 s C_{g s n}}{g_{m 2}}\right)+i_{4}\left(1+\frac{2 s C_{s g p}}{g_{m 4}}\right)\right]
\end{aligned}
$$

Substituting (3.8) into (3.10), the voltage of $v_{x}-v_{y}$ can be found as

$$
v_{x}-v_{y}=\frac{v_{n s}}{1+R_{s o} \sum_{k=1}^{4} g_{m k t z}}
$$

with $\quad g_{m 1,2 t z}=g_{m 1,2 t}\left(1+\frac{2 s C_{g s n}}{g_{m 1,2}}\right), g_{m 3,4 t z}=g_{m 3,4 t}\left(1+\frac{2 s C_{s g p}}{g_{m 3,4}}\right)$

The output differential noise current $i_{n s_{-} o u t}=i_{y}-i_{x}$ due to noise source of $v_{n s}$ can be calculated as

$$
i_{n s_{-} \text {out }}=\frac{-v_{n s} \sum_{k=1}^{4} g_{m k t}}{1+R_{s o} \sum_{k=1}^{4} g_{m k t z}}
$$

With similar procedure, we can calculate the output differential noise currents $i_{n M 1_{\_} \text {out }}$, $i_{n M 3 \_o u t}, i_{n R s p_{0} o u t}, i_{n R s n_{-} \text {out }}$ due to $v_{n M 1}, v_{n M 3}, v_{n R s p}$ and $v_{n R s n}$ respectively

$$
\begin{aligned}
i_{n M 3_{-} \text {out }}= & \frac{-R_{s o} v_{n M 3}\left[g_{m 3 t z}\left(1+R_{s p} s C_{s g p}\right)-2 s C_{s g p}\right] \sum_{k=1}^{4} g_{m k t}}{1+R_{s o} \sum_{k=1}^{4} g_{m k t z}} \\
& +v_{n M 3} g_{m 3 t}\left(1+R_{s p} s C_{s g p}\right) \\
i_{n M 1_{\_} \text {out }}= & \frac{-R_{s o} v_{n M 1}\left[g_{m 1 t z}\left(1+R_{s n} s C_{g s n}\right)-2 s C_{g s n} \sum_{k=1}^{4} g_{m k t}\right.}{1+R_{s o} \sum_{k=1}^{4} g_{m k t z}} \\
& +v_{n M 1} g_{m 1 t}\left(1+R_{s n} s C_{g s n}\right) \\
i_{n R s n_{-} \text {out }}= & \frac{R_{s o} v_{n R s n} g_{m 1 t}\left(1+\frac{2 s C_{g s n}}{g_{m 1}}\right) \sum_{k=1}^{4} g_{m k t}}{1+R_{s o} \sum_{k=1}^{4} g_{m k t z}}-v_{n R s n} g_{m 1 t} \\
i_{n R s p_{-} \text {out }}= & \frac{R_{s o} v_{n R s p} g_{m 3 t}\left(1+\frac{2 s C_{s g p}}{g_{m 3}}\right) \sum_{k=1}^{4} g_{m k t}}{1+R_{s o} \sum_{k=1}^{4} g_{m k t z}}-v_{n R s p} g_{m 3 t}
\end{aligned}
$$


Table 3.1: NF versus $(\gamma / \alpha)$ comparison of (3.4) and (3.22)

\begin{tabular}{|l|r|r|r|r|r|r|}
\hline$(\gamma / \alpha)$ & $2 / 3$ & 1 & 1.5 & 2 & 2.5 & 3 \\
\hline$N F_{\text {cross-coupling }}(\mathrm{dB})$ & 1.25 & 1.76 & 2.43 & 3.01 & 3.52 & 3.98 \\
\hline$N F_{\text {proposed }}(\mathrm{dB})$ & 0.88 & 1.07 & 1.34 & 1.59 & 1.83 & 2.06 \\
\hline$\Delta N F(\mathrm{~dB})$ & 0.37 & 0.69 & 1.09 & 1.42 & 1.69 & 1.92 \\
\hline
\end{tabular}

The same noise contribution will be achieved from the other half of the circuit. The noise factor $(\mathrm{F})$ and noise figure $(\mathrm{NF})$ will be calculated based on (3.13-3.17) as

$$
\begin{gathered}
F=\frac{2 i_{n s_{-} \text {out }}^{2}+2 i_{n M 1_{-} \text {out }}^{2}+2 i_{n M 3_{-} \text {out }}^{2}+2 i_{n R_{s n_{-} \text {out }}}^{2}+2 i_{n R s p_{-} \text {out }}^{2}}{2 i_{n s_{-} \text {out }}^{2}} \\
N F=10 \log _{10}(F)
\end{gathered}
$$

In order to compare NF of the proposed circuit to the one with conventional crosscoupling, the equivalent circuit can be simplified by ignoring the gate-source capacitances. The noise factor in this case will be

$$
\begin{aligned}
F= & 1+\frac{\gamma_{1} g_{m 1 t}^{2}}{\alpha_{1} g_{m 1} R_{s o}\left(\sum_{k=1}^{4} g_{m k t}\right)^{2}}+\frac{\gamma_{3} g_{m 3 t}^{2}}{\alpha_{3} g_{m 3} R_{s o}\left(\sum_{k=1}^{4} g_{m k t}\right)^{2}} \\
& +\frac{g_{m 1 t}^{2}}{\left(\sum_{k=1}^{4} g_{m k t}\right)^{2}} \frac{R_{s n}}{R_{s o}}+\frac{g_{m 3 t}^{2}}{\left(\sum_{k=1}^{4} g_{m k t}\right)^{2}} \frac{R_{s p}}{R_{s o}}
\end{aligned}
$$

The input impedance of the differential circuit ideally should be $Z_{i n}=2 R_{s o}$. Then for matching we need

$$
Z_{\text {in }}=2 R_{\text {so }}=\frac{2}{\sum_{k=1}^{4} g_{m k t}}
$$

For brevity we can assume that the differential circuit is perfectly balanced having the same $\gamma, \alpha$ values for all transistors. Then (3.20) can be simplified to

$$
F=1+\frac{\gamma\left(\frac{g_{m 1 t}^{2}}{g_{m 1}}+\frac{g_{m 3 t}^{2}}{g_{m 3}}\right)}{4 \alpha R_{s o}\left(g_{m 1 t}+g_{m 3 t}\right)^{2}}+\frac{\left(R_{s n} g_{m 1 t}^{2}+R_{s p} g_{m 3 t}^{2}\right)}{4 R_{s o}\left(g_{m 1 t}+g_{m 3 t}\right)^{2}}
$$

It should be noted that the double cross-coupling results in $1 / 4$ coefficient for the $(\gamma / \alpha)$ contribution as compared to $1 / 2$ for the traditional cross-coupling. Moreover, the noise factor contribution by the source degeneration resistors (the $3^{\text {rd }}$ term in (3.22)) appears less than the one by transistors for $(\gamma / \alpha)>1$. A comparison between NF of the proposed circuit and the conventional one (3.4) for $g_{m 1}=g_{m 2}=30 \mathrm{mS}, g_{m 3}=g_{m 4}=13.6 \mathrm{mS}, R_{s o}=$ $50 \Omega, R_{s n}=111 \Omega, R_{s p}=17 \Omega$, is shown in Table 3.1. With technology scaling the ratio 


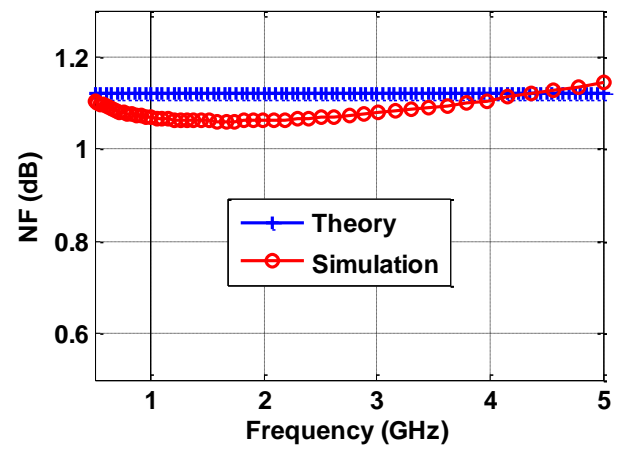

Figure 3.9 NF comparison of analytical model (3.13-3.19) and SpectreRF® circuit simulation for proposed LNTA (transistor level).

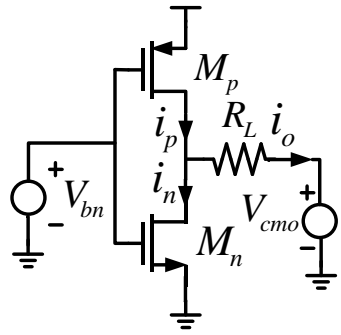

(a)

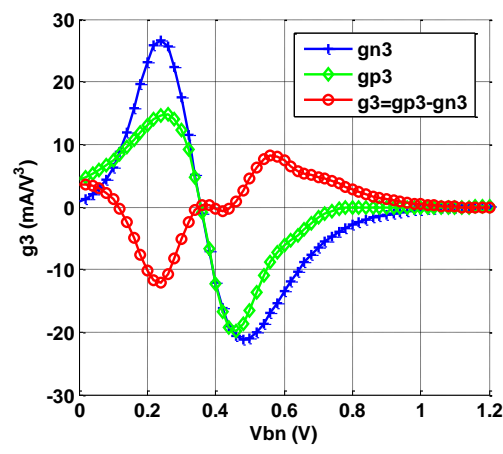

(b)

Figure 3.10 a) Schematic of conventional inverter, b) Simulation of third-order transconductances of PMOS $g_{3 p}$, NMOS $g_{3 n}$ and output $g_{3}$.

$(\gamma / \alpha)$ is increasingly large so the NF improvement is more pronounced. For example with $(\gamma / \alpha)=1.5$ the proposed LNTA can improve NF from $2.43 \mathrm{~dB}$ down to $1.34 \mathrm{~dB}$.

The NF comparison of the presented analytical model and SpectreRF® circuit simulation including the gate-source capacitances according to (3.13-3.19) is shown in Fig. 3.9. In this verification we use specifications captured from the designed chip: $g_{m 1}$ $=g_{m 2}=30 \mathrm{mS}, g_{m 3}=g_{m 4}=13.6 \mathrm{mS}, R_{s o}=50 \Omega, R_{s p}=17.2 \Omega, R_{s n}=110.8 \Omega, C_{g s n}=30$ $\mathrm{fF}, C_{g s p}=20 \mathrm{fF}$. As seen the respective differences remain within $0.08 \mathrm{~dB}$ that can be considered negligible. 


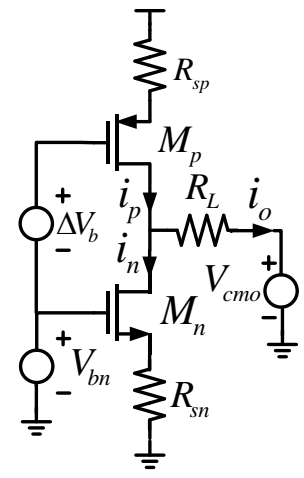

(a)

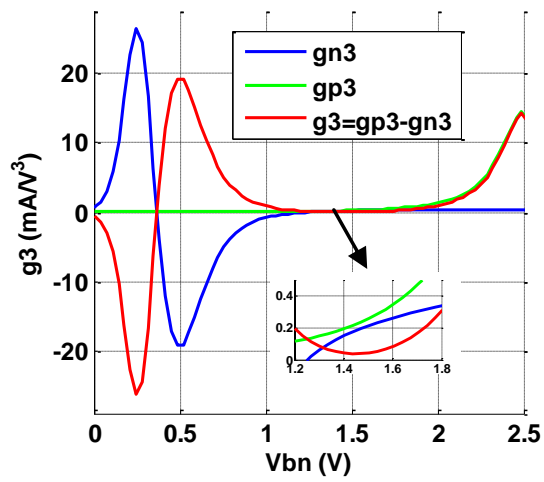

(b)

Figure 3.11: a) Schematic of resistive-feedback technique, b) Simulation of third-order transconductances of PMOS $g_{3 p}$, NMOS $g_{3 n}$ and output $g_{3}$.

\subsection{LNA Linearity Analysis Using Volterra Series}

The simulation environment using a conventional inverter, here, also considered as common-source complementary DS circuit, with output bias voltage was proposed in [22] as shown in Fig. 3.10a. This circuit can achieve high linearity due to subtraction of the nonlinear current components of the transistors $M_{p}$ and $M_{n}$. Both the second and third order terms can be partly cancelled if the circuit is appropriately biased. However, the useful input range is very narrow as shown for $g_{3}$ in Fig. $3.10 \mathrm{~b}$ where $g_{3}=\partial^{3} i_{o} / \partial\left(V_{i n}\right)^{3}$. In effect the possible blockers are not well tolerated by this circuit, still resulting in significant distortion.

A possible way to overcome this problem is using different bias voltages for $M_{p}$ and $M_{n}$ in combination with the resistive source degeneration applied to the both transistors as presented in Fig. 11a [26]. In Fig. 3.11b, the input voltage range can be significantly increased comparing the previous case in Fig. $3.10 \mathrm{~b}$. The combined $g_{3}$ is less than its components $g_{n 3}$ and $g_{p 3}$ in the operating range as seen in the zoom view. Moreover, it should be noted that $R_{s p}$ is much less than $R_{s n}$ in order to maintain the output bias voltage at $V_{d d} / 2$ while $M_{n}$ is larger than $M_{p}$. Should we increase the size of $M_{p}$ and the resistance of $R_{s p}$, the effective $g_{3}$ would be less, but its range would shrink degrading the linearity for large blockers.

The following analysis aims at describing IIP3 and third-order gain $\mathrm{H}_{3}$ of LNTA using the Volterra series approach. Fig. 3.12 shows the small-signal model for linearity analysis where the differential circuits are assumed to be identical for simplicity. The drain current of $M_{p}$ and $M_{n}$ can be modelled up to $3^{r d}$-order as

$$
i_{d p}=g_{1 p} v_{s g p}+g_{2 p} v_{s g p}^{2}+g_{3 p} v_{s g p}^{3}
$$




$$
i_{d n}=g_{1 n} v_{g s n}+g_{2 n} v_{g s n}^{2}+g_{3 n} v_{g s n}^{3}
$$

where $g_{i p}$ and $g_{i n}$ are the $i^{\text {th }}$-order coefficients of $M_{p}$ and $M_{n}$, accordingly, obtained by taking the derivative of the drain DC current $I_{S D} / I_{D S}$ with respect to the gate-source voltage $V_{S G} / V_{G S}$ at the $\mathrm{DC}$ bias point

$$
\begin{gathered}
g_{1 p}=\frac{\partial I_{S D P}}{\partial V_{S G P}}, g_{1 n}=\frac{\partial I_{D S N}}{\partial V_{G S N}} \\
g_{2 p}=\frac{1}{2 !} \frac{\partial^{2} I_{S D P}}{\partial V_{S G P}^{2}}, g_{2 n}=\frac{1}{2 !} \frac{\partial^{2} I_{D S N}}{\partial V_{G S N}^{2}} \\
g_{3 p}=\frac{1}{3 !} \frac{\partial^{3} I_{S D P}}{\partial V_{S G P}^{3}}, g_{3 n}=\frac{1}{3 !} \frac{\partial^{3} I_{D S N}}{\partial V_{G S N}^{3}}
\end{gathered}
$$

Applying the Volterra series to the output voltage

$$
\begin{aligned}
& v_{\text {outn }}=G_{1} \circ v_{\text {in }}+G_{2} \circ{v_{\text {in }}}^{2}+G_{3} \circ v_{\text {in }}^{3}, \\
& v_{\text {in }}=A_{1} \circ v_{\text {so }}+A_{2} \circ v_{\text {so }}{ }^{2}+A_{3} \circ v_{s o}{ }^{3}, \\
& v_{\text {out }}=H_{1} \circ v_{\text {so }}+H_{2} \circ v_{\text {so }}{ }^{2}+H_{3} \circ v_{\text {so }}{ }^{3},
\end{aligned}
$$

where $v_{\text {in }}=v_{\text {inp }}-v_{\text {inn }}$ and $v_{\text {out }}=v_{\text {outp }}-v_{\text {outn }}$. If circuits are completely symmetric $v_{\text {out }}$ can be calculated as

$$
\begin{aligned}
& v_{\text {outp }}=-G_{1} \circ v_{\text {in }}+G_{2} \circ v_{\text {in }}{ }^{2}-G_{3} \circ v_{\text {in }}{ }^{3}, \\
& v_{\text {out }}=-2 G_{1} \circ v_{\text {in }}-2 G_{3} \circ v_{\text {in }}{ }^{3} .
\end{aligned}
$$

From (3.28) and (A.3.15) from Appendix A, we have

$$
\begin{gathered}
G_{1}=\frac{-\hat{Z}_{L}\left\{\frac{\left(g_{1 p}+\frac{1}{r_{d p}}\right) n_{p}}{\left(1+k_{p}\right) m_{p}}+\frac{\left(g_{1 n}+\frac{1}{r_{d n}}\right) n_{n}}{\left(1+k_{n}\right) m_{n}}\right\}}{\eta_{L}} \\
G_{2}=\frac{\hat{Z}_{L}\left\{\frac{g_{2 p} G_{1 p}^{2}}{\left(1+k_{p}\right)^{3} m_{p}^{2}}-\frac{g_{2 n} G_{1 n}{ }^{2}}{\left(1+k_{n}\right)^{3} m_{n}^{2}}\right\}}{\eta_{L}}
\end{gathered}
$$




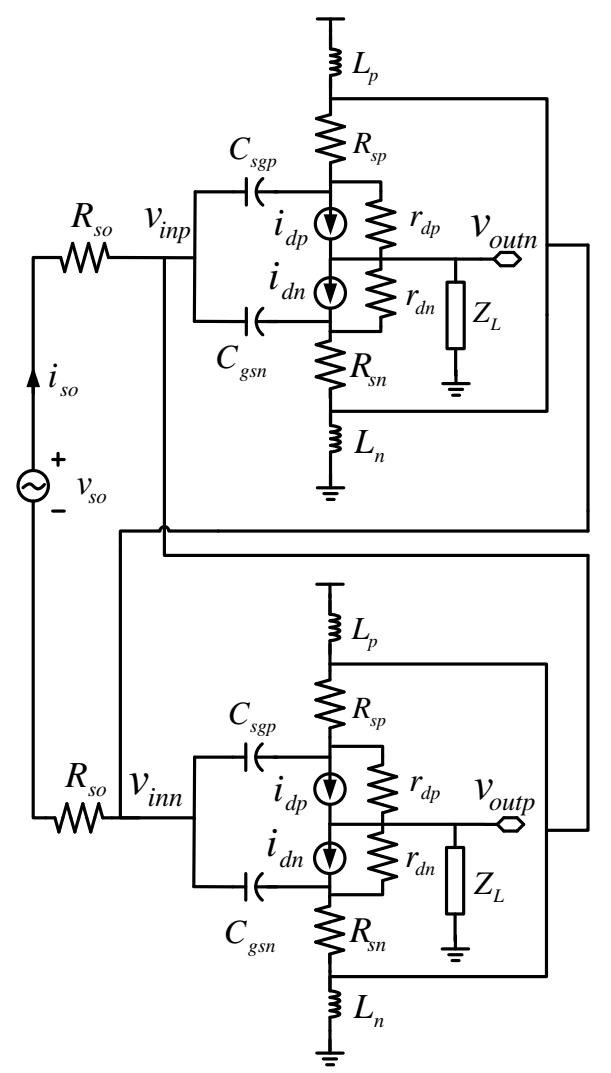

Figure 3.12: Equivalent circuit of a proposed wideband LNTA.

$$
\begin{gathered}
G_{3}=\frac{\frac{\hat{Z}_{L} G_{1 p}}{\left(1+k_{p}\right)^{3} m_{p}^{2}}\left\{2 g_{2 p} \frac{R_{s p}}{r_{d p}} G_{2}+\frac{G_{1 p}{ }^{2}}{\left(1+k_{p}\right)}\left[m_{p}\left[g_{3 p}-\frac{2 R_{s p} g_{2 p}^{2}}{\left(1+k_{p}\right) m_{p}}\right]\right\}\right.}{\eta_{L}} \\
\frac{-\frac{\hat{Z}_{L} G_{1 n}}{\left(1+k_{n}\right)^{3} m_{n}^{2}}\left\{2 g_{2 n} \frac{R_{s n}}{r_{d n}} G_{2}+\frac{G_{1 n}{ }^{2}}{\left(1+k_{n}\right) m_{n}}\left[g_{3 n}-\frac{2 R_{s n} g_{2 n}^{2}}{\left(1+k_{n}\right) m_{n}}\right]\right\}}{\eta_{L}}
\end{gathered}
$$

where 


$$
\begin{gathered}
\eta_{L}=1-\hat{Z}_{L}\left\{\frac{\left(g_{1 p}+\frac{1}{r_{d p}}\right) R_{s p}}{\left(1+k_{p}\right) m_{p} r_{d p}}+\frac{\left(g_{1 n}+\frac{1}{r_{d n}}\right) R_{s n}}{\left(1+k_{n}\right) m_{n} r_{d n}}\right\} \\
G_{1 p}=-n_{p}+\frac{R_{s p}}{r_{d p}} G_{1}, G_{1 n}=n_{n}-\frac{R_{s n}}{r_{d n}} G_{1}
\end{gathered}
$$

Substituting (A.3.17-A.3.22), (A.3.3), (A.3.9-A.3.13), (3.23-3.24) into (A.3.16) and comparing with (3.29), we can find $A_{1}, A_{2}$ and $A_{3}$

$$
\begin{aligned}
& A_{1}=\frac{1}{1+2 R_{s o}\left\{\frac{1}{2}\left(\frac{1}{s L_{p}}+\frac{1}{s L_{n}}-\frac{1}{r_{d p}}-\frac{1}{r_{d n}}\right)+G_{1}\left(\frac{1}{r_{d p}}+\frac{1}{r_{d n}}\right)+G_{1 p}\left[\frac{-2 s C_{g s p}-\frac{1}{r_{d p}}+\frac{m_{p}}{R_{s p}}}{\left(1+k_{p}\right) m_{p}}-\frac{1}{R_{s p}}\right]+G_{1 n}\left[\frac{2 s C_{g s n}+\frac{1}{r_{d n}}-\frac{m_{n}}{R_{s n}}}{\left(1+k_{n}\right) m_{n}}+\frac{1}{R_{s n}}\right]\right\}} \\
& A_{2}=2 R_{s o} A_{1}^{3}\left\{\frac{R_{s p} g_{2 p}}{\left(1+k_{p}\right)^{3} m_{p}^{3}}\left(\frac{1}{r_{d p}}-\frac{m_{p}}{R_{s p}}\right)\left(-n_{p}+\frac{R_{s p}}{r_{d p}} G_{1}\right)^{2}-\frac{R_{s n} g_{2 n}}{\left(1+k_{n}\right)^{3} m_{n}^{3}}\left(\frac{1}{r_{d n}}-\frac{m_{n}}{R_{s n}}\right)\left(n_{n}-\frac{R_{s n}}{r_{d n}} G_{1}\right)^{2}\right\} \\
& A_{3}=2 R_{s o} A_{1}^{2} \frac{s C_{g s p}}{\left(1+k_{p}\right) m_{p}}\left\{\left[\frac{R_{s p}}{r_{d p}} G_{3} A_{1}^{2}-\frac{2 A_{2} R_{s p} g_{2 p}}{\left(1+k_{p}\right)^{2} m_{p}^{2}} G_{1 p}{ }^{2}\right]+\frac{R_{s p} A_{1}^{2}}{\left(1+k_{p}\right)^{3} m_{p}^{3}}\left[\left(\frac{2 g_{2 p}^{2} R_{s p}}{\left(1+k_{p}\right) m_{p}}-g_{3 p}\right) G_{1 p}{ }^{3}\right]\right\} \\
& -2 R_{s o} A_{1}^{2} \frac{\left(s C_{g s p}+\frac{1}{r_{d p}}-\frac{m_{p}}{R_{s p}}\right)}{\left(1+k_{p}\right) m_{p}}\left\{\left[\frac{-R_{s p}}{r_{d p}} G_{3} A_{1}^{2}-\frac{2 A_{2} R_{s p} g_{2 p}}{\left(1+k_{p}\right)^{2} m_{p}^{2}} G_{1 p}{ }^{2}\right]-\frac{R_{s p} A_{1}^{2}}{\left(1+k_{p}\right)^{3} m_{p}^{3}}\left[\left(\frac{2 g_{2 p}^{2} R_{s p}}{\left(1+k_{p}\right) m_{p}}-g_{3 p}\right) G_{1 p}{ }^{3}\right]\right\} \\
& -2 R_{s o} A_{1}^{2} \frac{s C_{g s n}}{\left(1+k_{n}\right) m_{n}}\left\{\left[\frac{-R_{s n}}{r_{d n}} G_{3} A_{1}^{2}-\frac{2 A_{2} R_{s n} g_{2 n}}{\left(1+k_{n}\right)^{2} m_{n}^{2}} G_{1 n}{ }^{2}\right]+\frac{R_{s n} A_{1}^{2}}{\left(1+k_{n}\right)^{3} m_{n}^{3}}\left[\left(\frac{2 g_{2 n}^{2} R_{s n}}{\left(1+k_{n}\right) m_{n}}-g_{3 n}\right) G_{1 n}{ }^{3}\right]\right\} \\
& +2 R_{s o} A_{1}^{2} \frac{\left(s C_{g s n}+\frac{1}{r_{d n}}-\frac{m_{n}}{R_{s n}}\right)}{\left(1+k_{n}\right) m_{n}}\left\{\left[\frac{R_{s n}}{r_{d n}} G_{3} A_{1}^{2}-\frac{2 A_{2} R_{s n} g_{2 n}}{\left(1+k_{n}\right)^{2} m_{n}^{2}} G_{1 n}{ }^{2}\right]-\frac{R_{s n} A_{1}^{2}}{\left(1+k_{n}\right)^{3} m_{n}^{3}}\left[\left(\frac{2 g_{2 n}^{2} R_{s n}}{\left(1+k_{n}\right) m_{n}}-g_{3 n}\right) G_{1 n}{ }^{3}\right]\right\}
\end{aligned}
$$

If two single-ended circuits are identical, we substitute $(3.29,3.30)$ into $(3.32)$ and have

$$
H_{1}\left(\omega_{1}\right)=-2 G_{1}\left(\omega_{1}\right) A_{1}\left(\omega_{1}\right)
$$




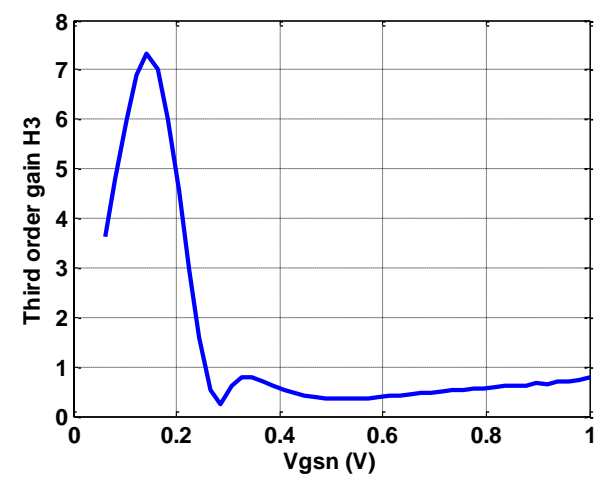

Figure 3.13: The third-order voltage gain $H_{3}(3.42)$ versus the bias voltage $V_{g s n}$.

$$
\begin{aligned}
H_{2}\left(\omega_{1}, \omega_{2}\right) & =-2 G_{1}\left(\omega_{1} \pm \omega_{2}\right) A_{2}\left(\omega_{1}, \omega_{2}\right) \\
H_{3}\left(\omega_{1}, \omega_{2}, \omega_{3}\right) & =-2\left[G_{1}\left(\omega_{1} \pm \omega_{2} \pm \omega_{3}\right) A_{3}\left(\omega_{1}, \omega_{2}, \omega_{3}\right)\right. \\
& \left.+G_{3}\left(\omega_{1}, \omega_{2}, \omega_{3}\right) A_{1}^{3}\left(\omega_{1} \pm \omega_{2} \pm \omega_{3}\right)\right]
\end{aligned}
$$

From [27, 34], IIP3 can be estimated as

$$
I I P_{3, d B m}=20 \log _{10}\left(\sqrt{\left|\frac{4}{3} \frac{H_{1}\left(\omega_{1}\right)}{H_{3}\left(\omega_{1}, \omega_{2}, \omega_{3}\right)}\right|}\right)+10
$$

DS technique has been used to cancel the third-order transconductance distortion $g_{3}$ well [18] but the operating range of input voltage $V_{g s}$ is not wider than $200 \mathrm{mV}$. In this design, we propose a technique that can cancel the third-order voltage gain (3.42) in larger operating range up to $650 \mathrm{mV}$ shown in Fig. 3.13. From that figure, the bias voltages can be chosen as $\mathrm{V}_{\mathrm{gsn}}=570 \mathrm{mV}$ and $\mathrm{V}_{\mathrm{sgp}}=\mathrm{V}_{\mathrm{gsn}}+190 \mathrm{mV}=760 \mathrm{mV}$. Therefore IIP3 of LNTA is not sensitive to the bias voltages and can tolerate large blockers up to $0 \mathrm{dBm}$.

The IIP3 obtained by the Volterra series model (3.43) and by SpectreRF ${ }^{\mathrm{TM}}$ simulations are depicted in Fig. 3.14 for two RF frequencies with the following parameters $g_{1 n}=30 \mathrm{mS}, g_{1 p}=13.6 \mathrm{mS}, g_{2 n}=57\left(\mathrm{~mA} / \mathrm{V}^{2}\right), g_{2 p}=8.2\left(\mathrm{~mA} / \mathrm{V}^{2}\right), g_{3 n}=-$ $70.3\left(\mathrm{~mA} / \mathrm{V}^{3}\right), g_{3 p}=-9.6\left(\mathrm{~mA} / \mathrm{V}^{3}\right), r_{d n}=339 \Omega, r_{d p}=843 \Omega$ at $V D D=2.5 \mathrm{~V}$ with $R_{s o}=$ $50 \Omega, L_{p}=30 \mathrm{nH}, L_{n}=70 \mathrm{nH}, R_{s p}=17.2 \Omega, R_{s n}=110.8 \Omega, C_{g s n}=30 \mathrm{fF}, C_{g s p}=20 \mathrm{fF}$. As shown, IIP3 is rising with the loading capacitance due to the reduced output voltage swing. For the same reason larger IIP3 values are attained at the higher operating frequency. It should be noted that the increment of IIP3 for $C_{\text {load }}$ increased from $0.2 \mathrm{pF}$ to $1 \mathrm{pF}(5 \times)$ at $f_{R F}=520 \mathrm{MHz}$ is almost the same as the one achieved for the frequency change from $520 \mathrm{MHz}$ to $3 \mathrm{GHz}$ (approx. $5 \times$ as well) for $C_{\text {load }}=0.2 \mathrm{pF}$. 


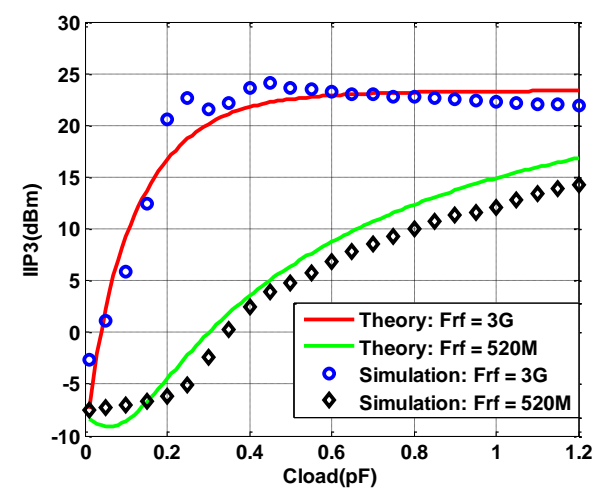

Figure 3.14: IIP3 comparison of analytical expression (3.43) and SpectreRF® simulation for LNTA, using two-tone $40 \mathrm{MHz}$ spacing (transistor level).

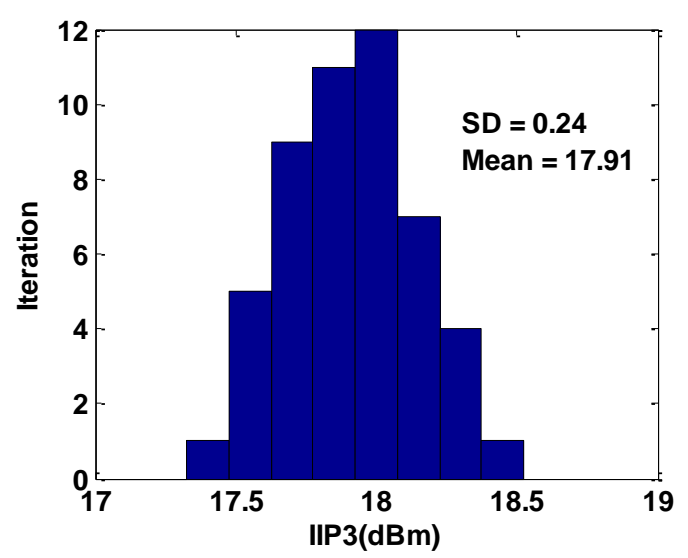

Figure 3.15: Monte-Carlo simulation of LNTA IIP3 obtained with 50 iterations at $f_{R F}=3 \mathrm{GHz}, 40 \mathrm{MHz}$ spacing, $C_{L}=1 \mathrm{pF}$.

In post-layout simulation with pad and bonding wire parasitics the IIP3 estimate at $f_{R F}$ $=3 \mathrm{GHz}$ with $40 \mathrm{MHz}$ spacing is reduced by $4 \mathrm{~dB}$, i.e. from $22 \mathrm{dBm}$ at transistor level to $18 \mathrm{dBm}$ for $2.5 \mathrm{~V}$ supply. The Monte-Carlo post-layout simulation under process variation with fixed bias is shown in Fig. 3.15. The mean value is $17.9 \mathrm{dBm}$ while the standard variation is only $0.24 \mathrm{~dB}$. To see the separate contributions to IIP3 by the different mechanisms used we found IIP3 to be reduced by $3 \mathrm{~dB}$, down to $15 \mathrm{dBm}$, for supply voltage changed to the standard value, $1.2 \mathrm{~V}$. The circuit will lose $6 \mathrm{~dB}$ more when the derivative superposition technique is excluded resulting in IIP3 $=9 \mathrm{dBm}$. Finally, by removing the resistive degeneration, IIP3 $=5 \mathrm{dBm}$ is attained. 


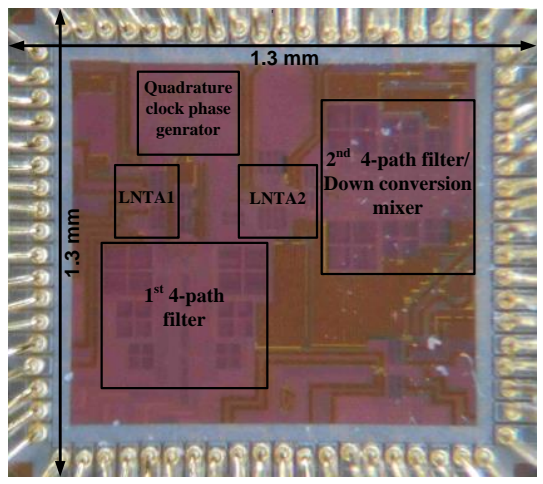

Figure 3.16: Chip photo [16].

Using a linear model also the LNTA transconductance estimate can be verified against the analytical model (3.6). From simulations over the operating frequency range with $Z_{L}<r_{d s}, G_{m}$ varying between $17-17.7 \mathrm{mS}$ can be found whereas from (3.6) it is around $18 \mathrm{mS}$. To reduce the effect of $r_{d s}$ on $G_{m}$ in this simulation a larger $C_{L}=4 \mathrm{pF}$ has been chosen.

\subsection{Implementation of a RF Selective Receiver Front-end}

The receiver front-end prototype was fabricated in $65 \mathrm{~nm}$ CMOS technology and the chip photo is shown in Fig. 3.16. A significant portion of the chip area is occupied by the banks of baseband capacitors $C_{B B}$, which allow for bandwidth programming. The maximum power consumption at $3 \mathrm{GHz}$ amounts for $113 \mathrm{~mW}$ and it drops to $46 \mathrm{~mW}$ at $0.5 \mathrm{GHz}$.

With N-path filter as a load the LNTA noise figure is raised by approximately $1 \mathrm{~dB}$ that can be attributed to noise folding as devised in [16]. In effect, the two-stage frontend NF varies between $3.2 \mathrm{~dB}$ and $5.2 \mathrm{~dB}$ for frequencies between $500 \mathrm{MHz}$ and 3 $\mathrm{GHz}$, respectively. The $\mathrm{NF}$ at $2 \mathrm{GHz}$ under $0 \mathrm{dBm}$ blocker with $100 \mathrm{MHz}$ offset is 12 $\mathrm{dB}$ that is below the 3GPP limit. Similarly, the in-band IIP3 is only less than $0 \mathrm{dBm}$ due to large loading impedance (large voltage swing). On the contrary, the out-of-band IIP3 is as large as $+20 \mathrm{dBm}$ in the lower frequency range and $+17 \mathrm{~dB}$ at $3 \mathrm{GHz}$. Additionally, superior blocker rejection of $44 \mathrm{~dB}$ is attained for frequencies up to $2 \mathrm{GHz}$ and $38 \mathrm{~dB}$ at $3 \mathrm{GHz}$ owing to the two-stage filtering [16]. Measured S11 for different LO frequencies is shown in Fig. 3.17. Within the whole frequency range $0.5-3 \mathrm{GHz}, \mathrm{S} 11$ is below -10 $\mathrm{dB}$ in the bandwidth of interest.

A comparison of the state-of-the-art and the presented LNTA design as well as the respective RF front-end based on N-path filtering is given in Table 3.2 and Table 3.3. In simulations the stand-alone amplifier compares favorably to the other work. Clearly, the LNTA design is critical for the performance of the measured front-end which, while 


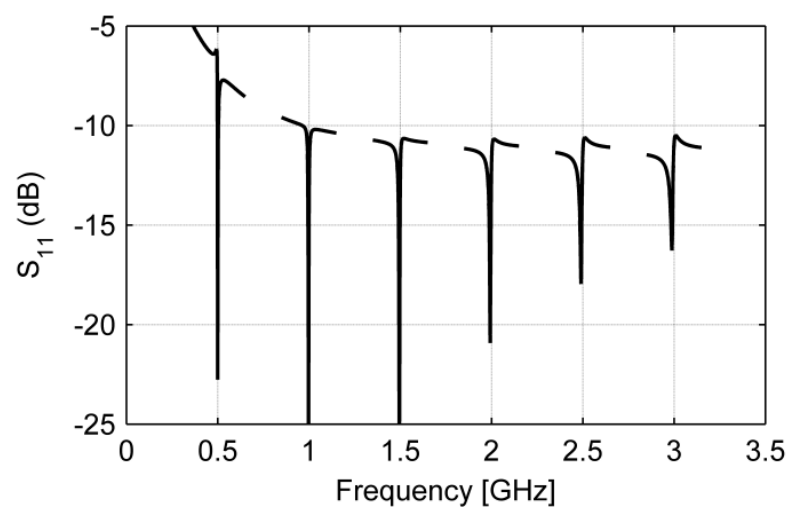

Figure 3.17: Measured $\mathrm{S}_{11}$ around LO frequencies for $C_{B B}=40 \mathrm{pF}$.

Table 3.2: Performance comparison of LNTA

\begin{tabular}{|c|c|c|c|c|}
\hline Author/ year & This work ${ }^{(* *}$ & $\begin{array}{l}\text { H.M. Geddada } \\
\text { [24] TMTT-14 }\end{array}$ & $\begin{array}{l}\text { M. Mehrpoo [70] } \\
\text { RFIC -13 }\end{array}$ & $\begin{array}{l}\text { L. Zhang [71] } \\
\text { TCASII-15 }\end{array}$ \\
\hline CMOS process & $65 \mathrm{~nm}$ & $45 \mathrm{~nm}$ & $65 \mathrm{~nm}$ & $65 \mathrm{~nm}$ \\
\hline NF (dB) & $1.3-1.9$ & $3 \mathrm{~min}$ & 5.9 & $6.2 \mathrm{~min}$ \\
\hline $\mathrm{Av}(\mathbf{d B}) / \mathbf{G m}$ & $14^{(\mathrm{b}}$ & $-1.7^{(\mathrm{a}}$ & $100 \mathrm{mS}$ & $242 \mathrm{mS}^{(*}$ \\
\hline IIP3 (dBm) & 18 & $10.8^{(\mathrm{a}}$ & 20 & 6.5 \\
\hline S11 (dB) & $<-11$ & $<-9$ & $<-10$ & $<-9$ \\
\hline Power (mW) & 16.5 & 30.2 & 11.3 & 72 \\
\hline BW (GHz) & $0.5-3$ & $0.1-2$ & $0.8-2.2$ & $0.6-10.5^{(*}$ \\
\hline
\end{tabular}

a) with (load) $\mathrm{Z}_{\mathrm{RF}}=30 \Omega,{ }^{\text {b) }}$ at $\left.2 \mathrm{GHz}, \mathrm{C}_{\mathrm{load}}=0.2 \mathrm{pF}(80 \Omega),{ }^{*}\right)$ Simulation results, ${ }^{* *)}$ Post-layout simulation with pad, bonding wire parasitics of $1 \mathrm{nH}$ and $2 \Omega$.

superior in terms of blocker rejection, can be found well in line with the remaining state-of-the-art specifications. 
Table 3.3: Performance comparison of Receiver Front-end

\begin{tabular}{|c|c|c|c|c|}
\hline Author/ year & This work & $\begin{array}{c}\text { D. Murphy [13] } \\
\text { JSCC-12 }\end{array}$ & $\begin{array}{c}\text { A. Mirzaei [72] } \\
\text { JSCC -11 }\end{array}$ & $\begin{array}{c}\text { M. Darvishi } \\
\text { [73] JSCC -13 }\end{array}$ \\
\hline CMOS process & $\mathbf{6 5 ~ n m}$ & $40 \mathrm{~nm}$ & $65 \mathrm{~nm}$ & $65 \mathrm{~nm}$ \\
\hline NF (dB) & $\mathbf{3 . 2}-\mathbf{5 . 3}$ & $1.9 /(5.5-8)^{(\mathrm{c})}$ & 5.3 & 2.8 \\
\hline Av(dB) /Gm & $\mathbf{4 5 - 2 5}$ & 58 & 55 & 25 \\
\hline IIP3 (dBm) & $\mathbf{2 0}$ & $+13 / 15^{(c)}$ & -6.3 & +26 \\
\hline S11 (dB) & $<-9$ & $<-8.8$ & $<-10$ & N/A \\
\hline Power (mW) & $\mathbf{4 6 - 1 1 3}$ & $50-100$ & 34.2 & $18-57$ \\
\hline BW (GHz) & $\mathbf{0 . 5}-\mathbf{3}$ & $0.8-2.9$ & 2.14 & $0.1-1.2$ \\
\hline
\end{tabular}

(c) Noise Cancellation ON/Noise cancellation OFF. 


\section{Chapter 4}

\section{Wideband RF Detector Design for On-Chip Test}

\subsection{Introduction}

The second work in Part I is design for on-chip test. This Chapter presents a design of an RF detector to be a part of a built-in test system suitable for RF measurements within a range 0.5 to $9 \mathrm{GHz}$ using $65 \mathrm{~nm}$ CMOS technology. The detector benefits from gain boosting mechanism and sub-ranging technique, which supported by high gain VGA, provides the dynamic range (DR) in excess of $60 \mathrm{~dB}$ and the minimum detectable signal (MDS) $<-50 \mathrm{dBm}$. Unlike in [41, 42] the detector input impedance is not compromised and at maximum frequency it is $>4 \mathrm{k} \Omega$ having a negligible effect on the circuit under test (CUT). The detector is intended for on-chip calibration to tolerate PTV variations and ensure good measurement accuracy [43]. These specifications put the presented design in line with the state-of-the-art and make it a good candidate for practical applications. 
On-chip RF detectors have been used for direct measurements of gain or $1 \mathrm{~dB}$ compression point $(1 \mathrm{dBcp})$ in receivers and transmitters over the last decade $[35,37$, $38,40,74-76]$. Also a measurement technique aimed at the intermodulation spec IP3, supported by spectral analysis of the RF detector response at low frequency, was presented [77]. Recently, sub-ranging detectors with increased gain and dynamic range have been proposed [41,80] and efforts toward more measurements with RF detectors were demonstrated, such as quadrature mismatch, RF isolation or intermodulation distortions (IMD) [42]. Since these measurements are sensitive to PVT variations and are used to derive the essential RF specs, calibration or adjustment of the detector characteristics appear imperative. In this case, keeping the respective calibration error within a few percent has been shown feasible [81(ch.3.3), 82, 78]. However, there has been no enough evidence on the accuracy of more demanding RF measurements, such as IMD.

It also presents a technique for IMD tests using on-chip RF detectors. We extend the calibration technique presented in [78] for RF detectors to serve also two-tone measurements aimed at IP3/IP2 specs, which are essential for RF frontend blocks [64]. The measurement technique is supported by a customized model of the circuit under test (CUT) since an RF detector cannot distinguish among different spectral components and for a given test the contribution of one spectral component can be obscured by another. To cope with this problem, the interfering components must be carefully identified for de-embedding the measurement. This comes at a price of more simulations preceding test, but the actual test time is not increased in this way. The measurement resolution must be high enough to distinguish among signal level increments introduced by nonlinear products (IMD and HD) of the CUT. This creates a challenge since the respective small differences decide the quality of test.

\subsection{RF Test Setup}

An example of a test setup exploiting embedded RF detectors for a SAW-less receiver under test [10] is shown in Fig. 4.1. The detectors have high input impedance and a large bandwidth to avoid loading and cover the receiver wide tuning range. The DC signal at each detector output is a measure of the RF amplitude at the corresponding circuit nodes. To achieve enough amplification the DC signal from each detector is routed through MUX 2-1 to the common VGA amplifier and next, to the T-ADC that is dedicated to test. Gain and selectivity of CUT can be measured in this setup directly. 


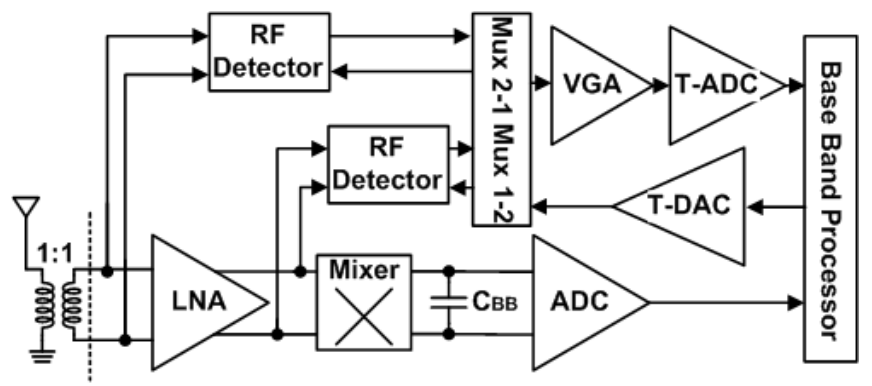

Figure 4.1: On-chip test setup for wideband SAW-less receiver.

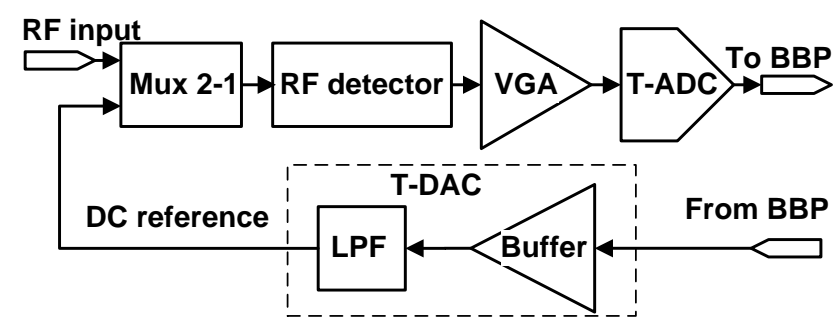

Figure 4.2: RF detector setup.

For calibration of the detectors a DC reference signal is provided by T-DAC as illustrated in Fig. 4.2. The T-DAC is implemented as one-bit sigma-delta modulator defined in software and its output signal is applied to a passive low-pass filter through a simple buffer. The accuracy of the reference signal is mainly subject to the supply voltage of the buffer and unlike other multi-bit DACs no correction for linearity is required in this case. The test circuitry is designed to be chip area efficient whereas its power consumption is not a primary objective. Importantly, the calibration process covers the complete measurement chain including MUX, VGA, and T-ADC, and not only of the RF detector.

In a real life scenario usually one detector on a chip of a production sample (say 5\%) can be characterized in terms of PVT variations using external RF stimuli (single tone with varied amplitude). The detector response to the RF- and the on-chip DC stimuli can be stored for comparison as shown in Fig.4.3. As these two output responses are correlated they can be used to identify RF responses of the remaining detectors in the production lot only using DC stimuli and a mapping function derived by statistical regression applied to the mentioned production sample. The resulting calibration error in the upper $6 \mathrm{~dB}$ signal range remains within a few percent by this procedure [78]. 


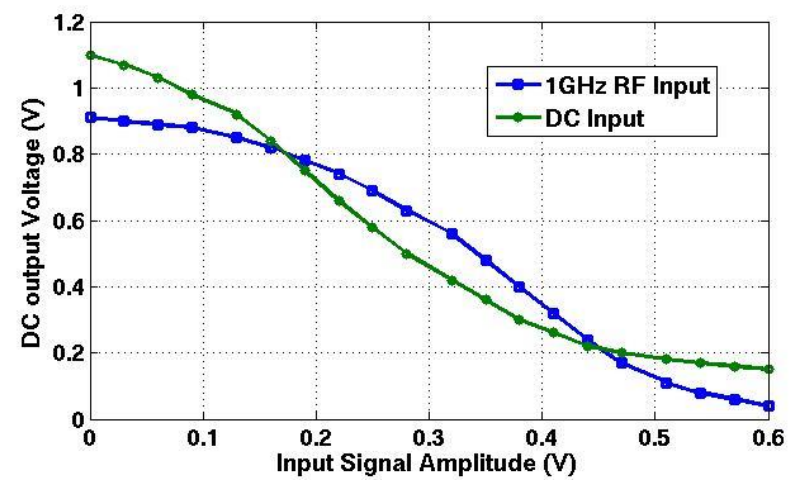

Figure 4.3: Response of RF detector to DC and one-tone input.

\subsection{RF Detector Design}

A new detection circuit is illustrated in Fig.4.4. Operating in saturation, M1 and M3 play a role of a differential detection device with tunable bias voltages: $V_{\text {biasn } 1}$ and $V_{\text {biasp }}$ to attain the wanted measurement sub-range. M2 generates a bias current for M3 and can be tuned by $V_{\text {biasn } 2}$ as well. The key solution here is the gain boosting loop provided by M4. This considerably helps to increase the detector gain (sensitivity) which otherwise is limited by small size of the MOS devices, necessary for high input impedance. Using this approach the sensitivity is enlarged by $12 \mathrm{~dB}$ with no effect on the input impedance as opposed to the circuits presented in [40, 42].

The value of the filtering capacitance $C_{L}$ should be high enough to filter out all RF products except for DC. For the DC output signal we just consider the effective resistive load $R_{\text {outp }}$ and $R_{\text {outn }}$ incident to nodes $V_{\text {outp }}$ and $V_{\text {outn }}$, respectively. For the saturation region the average of $V_{\text {outn }}$ can be calculated as

$$
\overline{V_{\text {outh }}}=R_{\text {outn }}\left[\frac{\mu_{p} C_{o x}}{2}\left(\frac{W}{L}\right)_{3}\left(\frac{V_{m x}^{2}}{8}+\left(V_{D D}-V_{\text {biasp }}-V_{\text {THP }}\right)^{2}\right)-I_{\text {biasn } 2}\right]
$$

where $V_{m x}$ is the input voltage amplitude. As shown in (4.1) the detection function is dependent on PTV variations but this issue can be resolved by calibration [43]. $V_{\text {outp }}$ is significantly amplified by gain boosting. The average of drain current $I_{D S 4}$ of $\mathrm{M}_{4}$ can be expressed as

$$
\overline{I_{D S 4}}=\frac{\mu_{p} C_{o x}}{2}\left(\frac{W}{L}\right)_{4}\left(V_{D D}-\overline{V_{o u t n}}-V_{T H P}\right)^{2}
$$

At the same time, $I_{D S 1}$ also detects the other RF input $V_{i n n}$ and its average is 


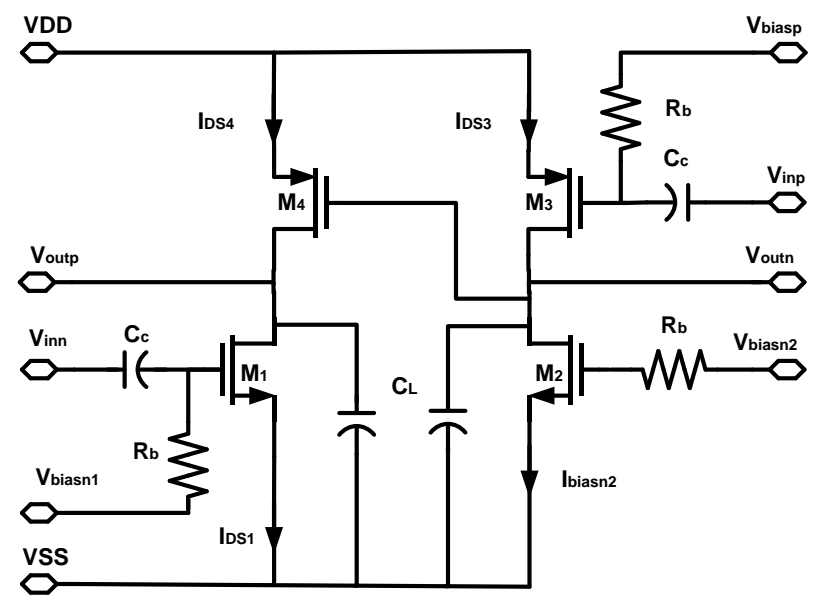

Figure 4.4: Proposed detection circuit.

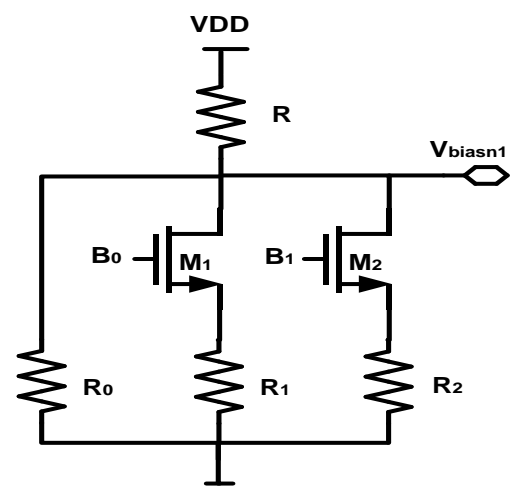

Figure 4.5: Two-bit bias circuit for sub-ranging operation.

$$
\overline{I_{D S 1}}=\frac{\mu_{n} C_{o x}}{2}\left(\frac{W}{L}\right)_{1}\left\{\frac{V_{m x}^{2}}{8}+\left(V_{\text {bias } n 1}-V_{T H N}\right)^{2}\right\}
$$

Likewise $V_{\text {outn }}$ the average of $V_{\text {outp }}$ is calculated using $R_{\text {outp }}$

$$
\overline{V_{\text {outp }}}=R_{\text {out }}\left(\overline{I_{D S 4}}-\overline{I_{D S 1}}\right)
$$

The achieved gain values $(>10 \mathrm{~dB}$ ) require sub-ranging operation. The two-bit bias circuit shown in Fig. 4.5 is used to generate different bias voltages of sub-ranges. Still it is not sufficient to attain a minimum detectable signal (MDS) and an input dynamic 
range suitable for various RF measurements [42] that motivates using VGA after the detection circuit. On the other hand, the VGA gain makes the requirements for the number of T-ADC bits reasonable. For input amplitudes $<1 \mathrm{mV}(<-50 \mathrm{dBm})$ and with $60 \mathrm{~dB}$ gain of VGA the detector total gain $A_{R F-D C}$ is approx. $18 \mathrm{~dB}$. Then the resolution corresponding to the MDS can be estimated from

$$
\Delta V=\frac{V_{D D}}{2^{N} A_{R F-D C}}
$$

where $N$ is the number of T-ADC bits. For $V_{D D}=1.2 \mathrm{~V}$ and $N=9$ we find $\Delta V=0.295$ $\mathrm{mV}(-60.6 \mathrm{dBm})$. Since the maximum possible signal is $V_{D D} / 2(5.5 \mathrm{dBm})$ the detector DR would be $66 \mathrm{~dB}$ as a preliminary estimate.

\subsection{RF Detector for Two-tone Test}

For IMD tests two-tone stimuli are required [64]. In this case a CMOS RF detector tends to produce a low-frequency spurious IM2 tone according to its inherent nonlinearity which, in fact, is responsible for the detection mechanism converting RF into DC voltage at its output. For a detector used in this work (Fig. 4.4) the integrated capacitors $C_{L}$ (in $\mathrm{pF}$ range) must be largely increased by off-chip capacitors to attenuate the spurious tone which otherwise tends to overload the following amplifier. For example, for a two-tone signal with spacing $4 \mathrm{MHz}$ and the capacitors $C_{L}$ increased from $2 \mathrm{pF}$ to $2 \mathrm{nF}$ the rejection of the IM2 tone is about $30 \mathrm{~dB}$ and it improves by 20 $\mathrm{dB} / \mathrm{dec}$ of the capacitor value.

The quadratic nonlinearity of the detector has diverse consequences for the two-tone operation. If the circuit devices stay in the saturation region the detector DC output only depends on the input signal power and there is no distinction in terms of the signal nature. In particular, in the one-tone or two-tone mode the respective transfer characteristics are the same for the RF signal power defined as the input variable, i.e.

$$
f_{1 t}\left(A_{1}^{2}\right)=f_{2 t}\left(2 A_{2}^{2}\right)
$$

where $A_{1}$ is the amplitude of one-tone, $A_{2}$ of each tone of the two-tone stimulus, and $A_{1}$ $=A_{2} \sqrt{ } 2$. For the presented detector with $A_{2}$ limited to $100 \mathrm{mV}$ the relation (1) holds with a small error $(<0.5 \%)$. However, for RF amplitudes $>100 \mathrm{mV}$ which are useful in IMD tests the error is largely increased. For this reason, calibration by a two-tone stimulus is necessary in this case but the same procedure as for one-tone can be used as well.

Like in (4.6), when other spectral components are present jointly with a two-tone, the detector DC response would be the same as for a "pure" two-tone if equal power is used in both cases:

$$
f_{2 t}\left(2 A_{2}^{2}+\sum_{k} B_{k}^{2}\right)=f_{2 t}\left(2 A_{2 p}^{2}\right)
$$




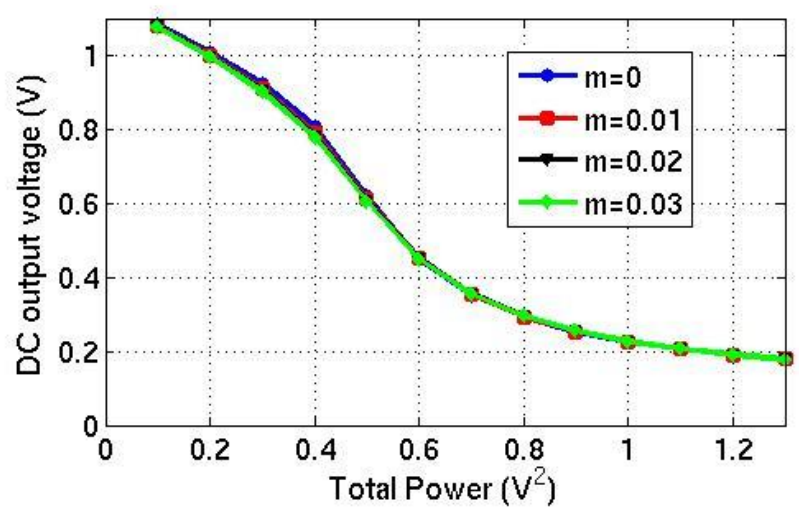

Figure 4.6: RF detector characteristics for two-tone input with IM3 tones.

This holds also for large signal power (beyond the mentioned limit) provided the other spectral components with amplitudes $B_{k}(k=1,2, \ldots)$ are small and are within the detector band. Clearly, in terms of two-tone IMD test the $B_{k}$ 's would be IMD and HD tones produced by CUT. On the other hand, high signal levels are of interest here, because they largely boost IMD/HD components to be seen as the corresponding DC increments at the detector output. We recall that the amplitudes of IM3/HD3 tones are proportional to the amplitude of the fundamental into power of three $\left(A_{I M 3} \propto A_{2}^{3}\right)$. In fact, in this approach we make use of the characteristics $f_{2 t}$ calibrated with a "pure" twotone stimulus to measure a two-tone response with IMD and also HD components produced by a CUT.

For preliminary verification of (4.7) we have applied a two-tone $2 \times A_{2}$ with four small $B_{k}$ tones, distributed like the corresponding IM3 tones, and next, compared against the reference response obtained with the equivalent "pure" two-tone of amplitude $A_{2 p}$. The resulting characteristics for different values of $m=4 B^{2} /\left(2 A_{2}^{2}\right)$ are shown in Fig. 4.6. For signal power $>0.5 \mathrm{~V}^{2}$ the respective differences at the output are within $5 \mathrm{mV}$ $(<1 \%)$ and drop when the signal power is increased. Compared to the basic definition we also find $m=2 I M 3$, so e.g. $I M 3=-20 \mathrm{~dB}$ corresponds to $m=0.02$.

\subsection{IP3/IP2 Test Technique}

In the two-tone test the detector output signal includes contributions by intermodulation and harmonic products (IMD, HD) of CUT that cannot be distinguished from the fundamental components directly. Additionally, the effect of gain compression should be taken into account when the input power to the CUT is largely increased to boost the IMD components. By using the calibrated characteristics of the detector we can estimate the actual signal power $P_{a c t}$ with a small error (assuming $m_{0}=0$ or other 


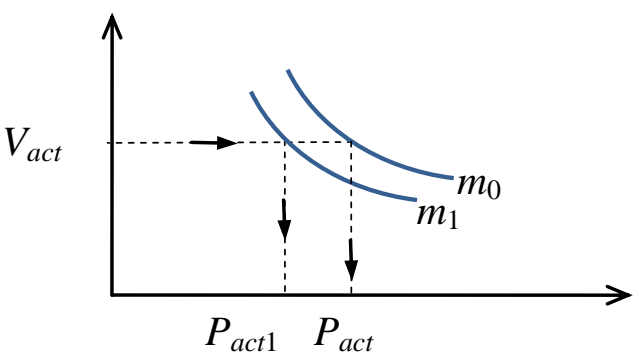

Figure 4.7: IMD measurement scheme.

value) and the respective "pure" two-tone power $P_{\text {fund }}$ (Fig. 4.7) if the gain of the CUT is also measured. For illustration of the test technique we use a common polynomial model of CUT defined by

$$
v_{\text {out }}=v_{i n}+a_{2} v_{i n}^{2}+a_{3} v_{\text {in }}^{3}
$$

where $v_{\text {in }}$ is a two-tone RF stimulus with amplitudes equal $A$, while $v_{\text {out }}$ is the corresponding output signal composed of the amplified two tones (fundamental) and additionally IMD and HD tones (two IM2 tones, two HD2, four IM3 tones, and two HD3 tones). For simplicity we assume the linear gain is equal one. Then the fundamental component power can be defined as $P_{\text {fund }}=2 A^{2}$ while the actual output power

$$
P_{a c t}=P_{\text {fund }}(1-c)^{2}+P_{I M 2}+2 P_{H D 2}+4 P_{I M 3}+2 P_{H D 3}
$$

where we assume the low frequency IM2 tone is blocked by a coupling capacitor between CUT and the detector and it has no effect on the measurement. The spectral components are denoted by $P_{I M 2}=\left(a_{2} A^{2}\right)^{2}, P_{H D 2}=\left(0.5 a_{2} A^{2}\right)^{2}, P_{I M 3}=\left(0.75 a_{3} A^{3}\right)^{2}, P_{H D 3}=$ $\left(0.25 a_{3} A^{3}\right)^{2}$, and $c=2.25\left|a_{3}\right| A^{2}[64, \mathrm{ch} .2]$.

The second order nonlinearity of CUT can be measured by the low frequency IM2 tone, retrieved from $v_{\text {out }}$ by a LPF, bypassing the detector as shown in Fig. 4.8. This filter needs to be carefully designed to minimize the effect of manufacturing variations on the cut-off frequency and hence, on the gain, which can badly affect the measurement accuracy. After digitization the respective IM2 amplitude $P_{I M 2}$ can be easily found. From the basic relations we also have $P_{H D 2}=0.25 P_{I M 2}$. 


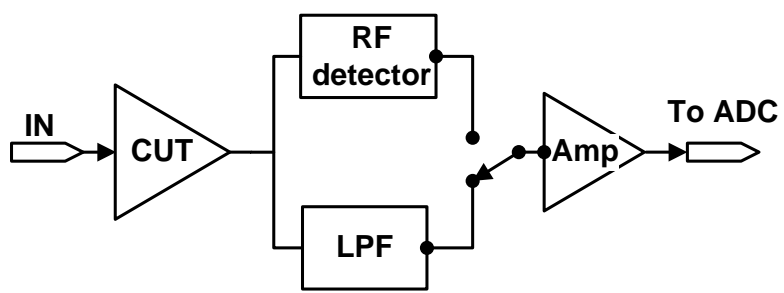

Figure 4.8: IM2 test setup.

Having identified the second order nonlinearity of the CUT, $P_{I M 3}$ can be calculated from (4.9) knowing that $P_{H D 3}=P_{I M 3} / 9$ and $c^{2}=0.5 P_{I M 3} / P_{\text {fund }}$.

Finally, a correction in terms of the $m$ value can be done. For this purpose, we can calculate the compressed two-tone power $P_{\text {comp }}$ (the first term in (4.9)) and the remaining IMD and HD power as $P_{\text {dist }}=P_{\text {act }}-P_{\text {comp }}$. Next, using $m_{1}=P_{\text {dist }} / P_{\text {comp }}$ from the characteristics shown in Fig. 4.7 the corresponding $P_{a c t 1}$ value can be found. A new value of $P_{I M 3}$ will follow accordingly. If necessary, this procedure can be repeated in a few iterations. Finally, the IP3/IP2 can be estimated from the basic formulas

$$
P_{I P 3}=\sqrt{0.125 P_{\text {fund }}^{3} / P_{I M 3}}, \quad P_{I P 2}=0.25 P_{\text {fund }}^{2} / P_{I M 2}
$$

but as it is a "one point" measurement, for more accuracy the test can be repeated for different values of the input amplitude. In simulations the accuracy of IP3 test for the polynomial-based CUT is practically only limited by the calibration error of the detector.

We also note that the gain of CUT can be measured by one-tone stimulus using small enough signal power to avoid compression. The contribution of HD terms can be usually neglected since HD2 $\propto A^{4}$ and HD3 $\propto A^{6}$.

For a practical CUT, like LNA, the model of nonlinearity should be customized. For this purpose, using Monte-Carlo simulation of the CUT model we find correlation among the IMD and HD components including also the gain compression. In particular, due to limited LNA bandwidth the IMD/HD tones at lower and higher frequencies would differ in power, unlike for the polynomial model in (4.9). Also the relations between IMD and HD terms would be different and in calculations they should be modified accordingly. Based on this the actual measured power can be expressed like (4.9) but with modified coefficients

$$
P_{a c t}=P_{\text {fund }}(1-c *)^{2}+h_{I M 2} P_{I M 2}+h_{H D 2} P_{H D 2}+h_{I M 3} P_{I M 3}+h_{H D 3} P_{H D 3}
$$

The actual IP3 and IP2 estimates for the CUT can be obtained from (4.10) as well. 


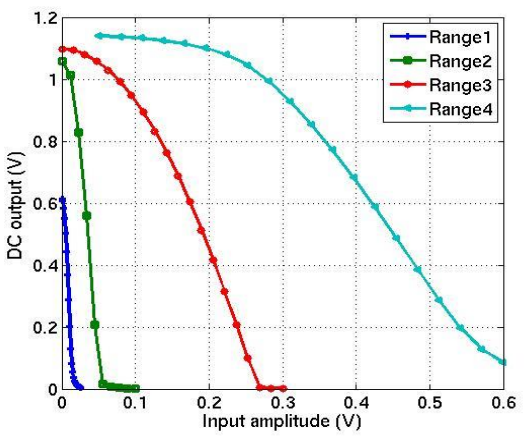

(a)

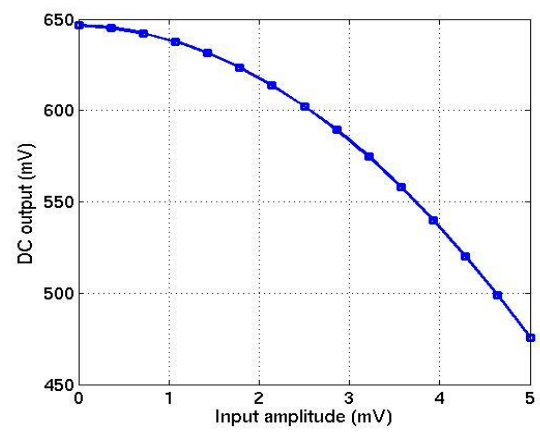

(b)

Figure 4.9: Detector characteristic using sub-ranges (a), and in Range 1 (b).

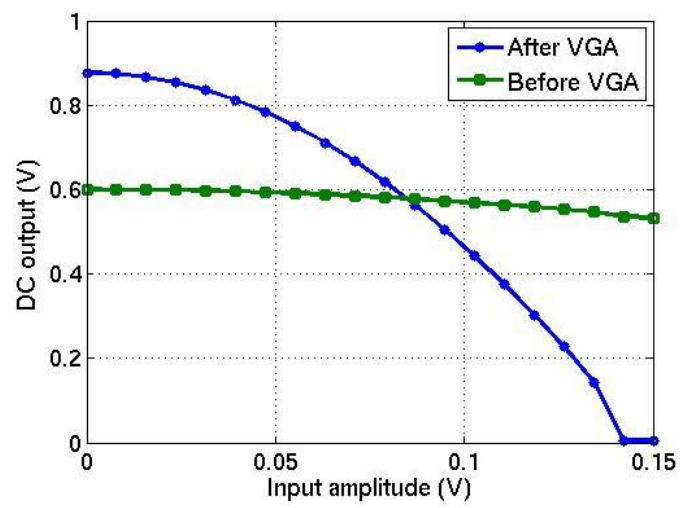

Figure 4.10: Comparison detector signal before and after VGA.

\subsection{Simulation Results}

The circuit has been simulated using Cadence ${ }^{\mathrm{TM}}$ software with RF transistor models (65 nm CMOS). The detector characteristics are shown in Fig. 4.9a. Four sub-ranges are used, each with different VGA gain between 0 and $66 \mathrm{~dB}$, suitable to cover the entire input range with a gain larger than $1 \mathrm{LSB} / \mathrm{MDS}$. The low range (Range 1) characteristic obtained with the maximum VGA gain $(66 \mathrm{~dB})$ is also shown in Fig. $4.9 \mathrm{~b}$. The detector gain drops when the input amplitude is reduced that is compliant with the analytical model discussed in Section 4.3. The detailed gain values are given in Table 4.1. By acknowledging the gain value for the input amplitude $<0.4 \mathrm{mV}$ to be $15.6 \mathrm{~dB}$, from (4.10) we find $\Delta V=0.38 \mathrm{mV}(-58 \mathrm{dBm})$, which can be considered the actual value of MDS with the corresponding $\mathrm{DR}=63 \mathrm{~dB}$. 


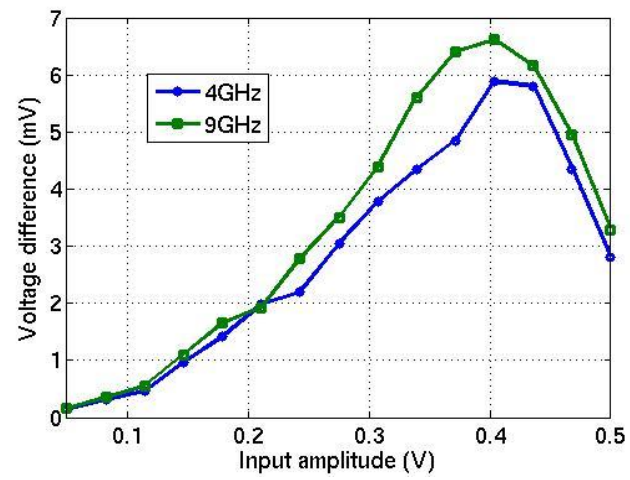

Figure 4.11: Output voltage difference compared to $0.5 \mathrm{GHz}$ operation.

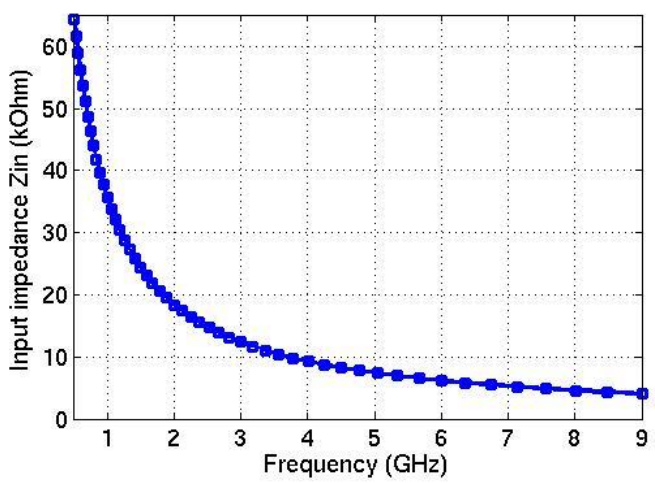

Figure 4.12: The input impedance vs. frequency.

The effect of VGA gain $(20 \mathrm{~dB})$ is illustrated in Fig. 4.10 showing that the VGA virtual ground is approx. $580 \mathrm{mV}$ (cross-point). The detector characteristics do not change much with the input frequency. The respective differences for operation at 4 $\mathrm{GHz}$ and $9 \mathrm{GHz}$ vs. $0.5 \mathrm{GHz}$ are illustrated in Fig. 4.11. The maximum difference amounts for $0.8 \%$. Fig. 4.12 illustrates the dependence of input impedance on frequency. In practice, it is equivalent to a capacitance of $8.8 \mathrm{fF}$ that gives more than $4 \mathrm{k} \Omega$ at $9 \mathrm{GHz}$. Finally, Table 4.2 gives a comparison of this work with other publications. The power consumption, $1.48 \mathrm{~mW}$ of the detector and $16 \mathrm{~mW}$ of VGA, is not critical in this application because they are only used during test. The layout area is more significant and this test circuitry including SAR T-ADC [85] occupies about $0.057 \mathrm{~mm}^{2}$ that is less than $6 \%$ of $1 \mathrm{~mm}^{2}$ chip area. Also the advantage in terms of the MDS and DR is evident. 
Table 4.1: RF Detector Gain vs Input Amplitude

\begin{tabular}{cccc}
\hline $\begin{array}{c}\text { Input } \\
\text { signal (mV) }\end{array}$ & $\begin{array}{c}\text { Input signal } \\
(\mathbf{d B m})\end{array}$ & $\begin{array}{c}\text { Detection } \\
\text { Circuit Gain } \\
(\mathbf{d B})\end{array}$ & $\begin{array}{c}\text { Total Gain } \\
(\mathbf{d B})\end{array}$ \\
\hline 0.38 & -58.4 & -51.2 & 15.6 \\
\hline 0.5 & -56 & -48.1 & 18.4 \\
\hline 1 & -50 & -44.2 & 22.3 \\
\hline 2 & -44 & -37.8 & 28.7 \\
\hline 3 & -40.5 & -34.2 & 32.3 \\
\hline 4 & -38 & -31.6 & 34.8 \\
\hline 5 & -36 & -30.2 & 36.2 \\
\hline
\end{tabular}

Table 4.2: Performance Comparison

\begin{tabular}{|c|c|c|c|c|c|c|c|}
\hline Paper/Year & $\begin{array}{c}\text { CMOS } \\
\text { process } \\
(\mathbf{n m})\end{array}$ & $\begin{array}{l}\text { Gain } \\
\text { (dB) }\end{array}$ & $\begin{array}{l}\text { Dynamic. } \\
\text { range } \\
\text { (dB) }\end{array}$ & $\begin{array}{c}\text { Min. } \\
\text { Detectable } \\
\text { Signal } \\
(\mathbf{d B m})\end{array}$ & $\begin{array}{c}\text { Input } \\
\text { impedance. }\end{array}$ & $\begin{array}{c}\text { Power } \\
(\mathrm{mW})\end{array}$ & $\begin{array}{c}\text { BW } \\
(\mathbf{G H z})\end{array}$ \\
\hline [38] 2008 & 350 & 23 & $>30$ & -25 & $13 \mathrm{fF}$ & 8.6 & $0.9-2.4$ \\
\hline [40] 2006 & 180 & 0 & $60^{(2}$ & N/A & $\begin{array}{l}663 \Omega \\
(12 \mathrm{fF})\end{array}$ & $0.01^{(2}$ & 20 \\
\hline [42] 2010 & 180 & 20 & N/A & N/A & N/A & 0.8 & $0.5-9$ \\
\hline This work & 65 & $80^{(1}$ & 63 & -58 & $\begin{array}{c}>4 \mathrm{k} \Omega \\
(8.8 \mathrm{fF})\end{array}$ & $1.48^{(3}$ & $0.5-9$ \\
\hline
\end{tabular}




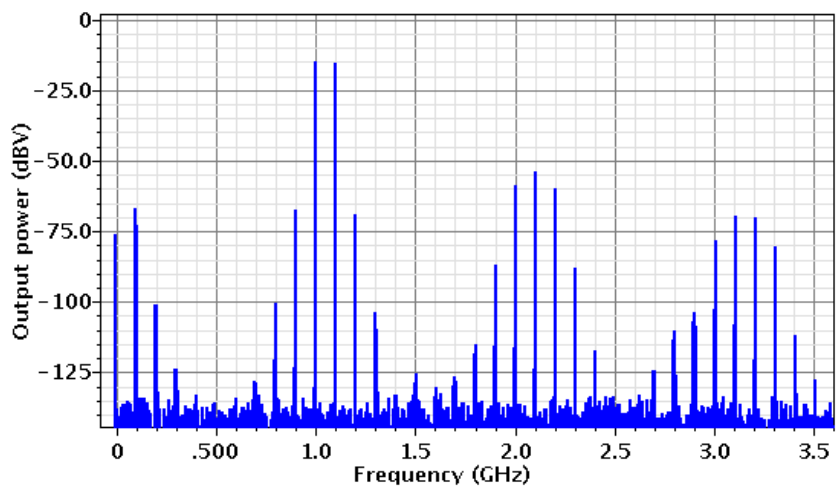

Figure 4.13: LNA spectrum for unloaded output and $P_{i n}=-35 \mathrm{dBm}$.

A low noise amplifier (LNA) [26] is used as the CUT. The LNA output spectrum for two-tone test with large spacing (for clarity) is illustrated in Fig. 4.13. The higher spectral components are practically negligible (some $30 \mathrm{~dB}$ below the $2^{\text {nd }}$ and $3^{\text {rd }}$ terms). For various output loads the LNA can achieve different gain and IP3 values useful to demonstrate the proposed measurement technique over $-10 \mathrm{dBm}$ to $0 \mathrm{dBm}$ IIP3 range.

Also a small mismatch is introduced into the circuit to attain IIP2 of approx. $25 \mathrm{dBm}$ that can be measured by the low frequency $P_{I M 2}$ tone (Fig. 4.8). By spectral analysis of the LNA model, we identify the coefficients $h$ defined in (4.11), and also the ratios between the respective IMD and HD components. The LNA is tested at $1 \mathrm{GHz}$ by a twotone with $4 \mathrm{MHz}$ spacing. For the accuracy we have to boost the distortion components by pushing the LNA into compression $\left(P_{\text {in }}>P_{1 \mathrm{~dB}}\right)$. For the gain value $G=19.89 \mathrm{~dB}, P_{\text {in }}$ $=-13 \mathrm{dBm}(A=70.71 \mathrm{mV})$, and $I I P 2=26.5 \mathrm{dBm}$ by Monte-Carlo simulation and regression technique we find: $c^{*}=\alpha\left(P_{H D 3} / P_{i n}\right)^{1 / 2}=0.37$ and $h_{I M 2}=0.32, h_{H D 2}=1.87$, $h_{I M 3}=2.76, h_{H D 3}=1.99$. Besides we find $P_{H D 2} / P_{I M 2}=0.004, P_{H D 3} / P_{I M 3}=0.1474$, and $P_{I M 2}=2.665 \mathrm{e}-4 \mathrm{~V}^{2}$. Then for $m_{0}=0$ we have $P_{a c t}=0.552 \mathrm{~V}^{2}$. From $(4.11)$ we calculate $P_{I M 3}=0.0134 \mathrm{~V}^{2}$ and $m_{1}=0.014$. After correction $P_{a c t 1}=0.549 \mathrm{~V}^{2}$ and $P_{I M 3}=0.0109 \mathrm{~V}^{2}$. The LNA IIP3 and IIP2 are calculated from (4.10) using $P_{\text {fund }}=2 P_{\text {in }}$ and the input referred $P_{I M 3}$ and $P_{I M 2}$ values.

Table 4.3 gives a comparison of three methods for IIP3 measurement. In the PSS (Periodic Steady State Analysis) the circuit IIP3 is measured from many points by sweeping input power so this technique tends to be more accurate. As seen, the measurements by the $\mathrm{RF}$ detector over $-10 \mathrm{dBm}$ to $0 \mathrm{dBm}$ range are within $0.8 \mathrm{~dB}$ error compared to the other two techniques. 
Table 4.3: Comparison of IIP3 Measurement Techniques

\begin{tabular}{ccccc}
\hline \multirow{2}{*}{$\begin{array}{c}\text { LNA output } \\
\left(\mathbf{V}_{\mathbf{p}-\mathbf{p}}\right)\end{array}$} & LNA gain & \multicolumn{3}{c}{ IIP3 $(\mathbf{d B m})$} \\
\cline { 3 - 5 } & $(\mathbf{V} / \mathbf{V})$ & $\begin{array}{c}\text { Transient } \\
+ \text { FFT }\end{array}$ & PSS & RF detector \\
\hline 0.73 & 15.33 & -9.04 & -9.5 & -8.56 \\
\hline 0.76 & 13.43 & -4.52 & -4.5 & -4.98 \\
\hline 0.77 & 9.87 & -0.31 & 0.2 & 0.53 \\
\hline
\end{tabular}

In this case, we cancalculate the necessary $\mathrm{T}-\mathrm{ADC}$ resolution as

$$
\Delta V=V_{D D} 2^{-N} / K \text {, }
$$

where $K$ is a voltage gain of the detector chain. For maximum IIP3 $=0 \mathrm{dBm}$ and $P_{\text {in }}=-$ $13 \mathrm{dBm}$, we have $P_{I M 3, \text { out }}=\left(P_{\text {in }}+G\right)-2\left(I I P 3-P_{\text {in }}\right)=-29 \mathrm{dBm}\left(11.22 \mathrm{mV}_{\mathrm{p}}\right)$. Assuming the accuracy of IIP3 be around $0.2 \mathrm{~dB}$, we have the corresponding $\Delta P_{I M 3, \text { out }}=0.4 \mathrm{~dB}$ and $P_{I M 3, \text { out } \text { min }}=-29.4 \mathrm{dBm}\left(10.72 \mathrm{mV}_{\mathrm{p}}\right)$ so using $\mathrm{V}^{2}$ scale $\Delta P_{I M 3, \text { out }}=(11.22 \mathrm{e}-$ $3)^{2}-(10.72 \mathrm{e}-3)^{2}=10.97 \mathrm{e}-6 \mathrm{~V}^{2}$. Then from (6) $\Delta P_{\text {act }}=(0.3+2.76+0.3) \Delta P_{I M 3, \text { out }}=$ $36.9 \mathrm{e}-6 \mathrm{~V}^{2}$ that according to the detector gain corresponds approx. to $\Delta V=40 \mu \mathrm{V}$. The $\mathrm{ADC}$ resolution depends on the gain $K$, for $K=40 \mathrm{~dB}(100)$ and $V_{D D}=1.2 \mathrm{~V}$ from (4.12) we find $N=9$. 


\section{Part II}

\section{Design of High Speed SC DACs for 60- GHz radio baseband on Transmitter Side}




\section{Chapter 5}

\section{Design and Analysis of High Speed Capacitive Pipeline DACs}

\subsection{Introduction}

Part II of thesis is design of high speed capacitive DACs for wideband wireless transmitters. The design and analysis of pipeline DACs is the first work of this part and is described in this Chapter. The second work of this part is design and implementation of a 12-bit split-segmented SC DACs which will be presented in Chapter 6.

Among D/A converters (DACs), recently, the current-steering architectures [53, 54, $86,87]$ have been mainly focused on due to their simplicity and the achieved good performances. Unlike this, the capacitive DACs (SC DACs) have not drawn much attention except for two recent publications [61, 62].

In fact, SC DACs have some advantages over the current-steering architectures [61, 62]. Specifically, as capacitor arrays they are easier to match that makes SC DACs 
virtually more linear. For $n$-bit resolution a pipeline SC DAC only requires $n$ identical stages as opposed to $2^{n}$ unit cells in a current steering DAC. Also the power consumption is low because their operation is based on charge redistribution. On the other hand, the ability to drive off-chip load of SC DACs is poor, so a suitable output driver is necessary. The driver appears critical for linearity of a capacitive DAC unless the required output voltage swing is low. One solution to this problem is using a closed-loop architecture where the circuit linearity is improved by negative feedback [61]. This technique, however, cannot work well at high frequencies $(>1 \mathrm{GHz})$. Another solution is an openloop driver design that can be supported by interleaving and a pre-distortion technique [62]. However, the latter is usually limited by an assumption of minimum memory effect of the nonlinear object.

This Chapter presents design of a simple pipeline SC DAC with a highly linear output driver using $65 \mathrm{~nm}$ CMOS technology. The main objective is achieving maximum data rate in this architecture. The circuit is optimized under linearity and noise constraints imposed by the capacitor array and the output driver. We define the design area of the SC array in terms of unit capacitance and the switch size for given resolution and clock frequency. The SFDR and SNR analysis shows that the DAC bandwidth limit is subject to incomplete settling and feed-through effects rather than $\mathrm{kT} / \mathrm{C}$ noise and the capacitor mismatch. A tradeoff between the DAC bandwidth and resolution accompanied by SFDR is demonstrated.

The DAC output driver is an open-loop wideband amplifier patterned after [100]. To attain high linearity a combination of the derivative superposition $[18,20]$ and resistive source-degeneration technique is used. We estimate the driver nonlinearity by using the Volterra series model [101]. The analysis verified by simulation shows that for signal bandwidth up to $5 \mathrm{GHz}$ and the output voltage swing $300 \mathrm{mV}_{\mathrm{pp}}$, HD3 better than $-72 \mathrm{~dB}$ is attained that corresponds to 12 bit resolution. This shows the contribution of the SC array to HD3 and SFDR of the SC DAC to be dominant.

Simulation results of the complete SC DAC with 8-bit resolution and Nyquist sampling show SFDR of $45 \mathrm{~dB}$ for $1.36 \mathrm{GHz}$ signal bandwidth. For 6-bit resolution and signal bandwidth of $5.5 \mathrm{GHz}$, SFDR of $33 \mathrm{~dB}$ is attained. The respective maximum data rate of $72 \mathrm{Gbps}$ compares favorably with the state-of-the-art.

\subsection{Capacitive DAC Architecture}

The pipeline capacitive DAC architecture shown in Fig. 5.1 was firstly proposed in [96]. The 12-bit input is divided into 4 segments of three-bits each. It needs one cycle of clock to complete 3-bit conversion in the 3-bit SC circuits. The 3-bit DFFs are employed to synchronize the signal using a non-overlapping three-phase clock. The output driver serves as a buffer driving an off-chip standard load. 


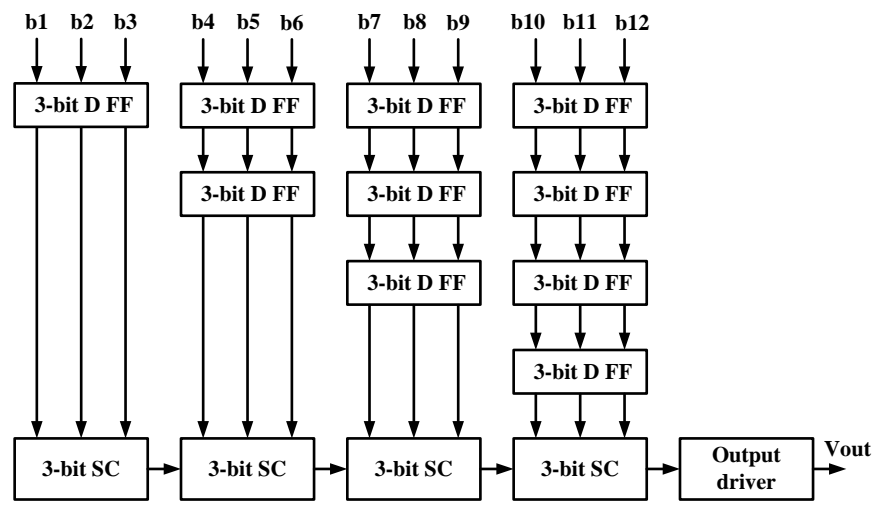

Figure 5.1: Pipeline SC DAC architecture.

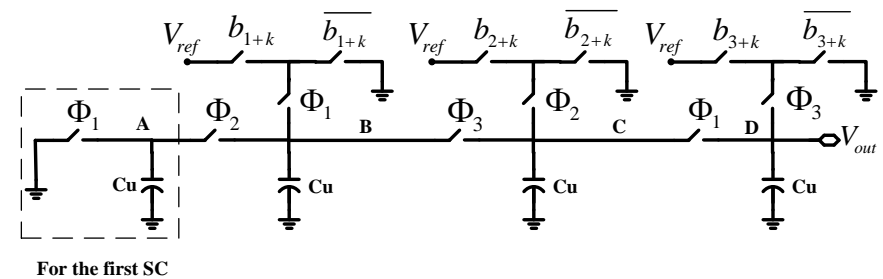

Figure 5.2 Three-bit switched capacitor segment.

Fig. 5.2 depicts the 3-bit SC segment where a three-phase clock $\left(\Phi_{1}, \Phi_{2}\right.$ and $\left.\Phi_{3}\right)$ is used. In the first SC segment, an extra switch is necessary to reset voltage at node A in the pre-charge step. During the time when $\Phi_{1}=1$, the capacitor at A is discharged to zero and the capacitor at B is charged either to $V_{r e f}$ if $b_{1+k}=1$ or discharged if $b_{1+k}=0$. When $\Phi_{1}$ goes low and $\Phi_{2}$ goes high, the voltages at $\mathrm{A}$ and $\mathrm{B}$ are equal to $V_{r e f} b_{1+k} / 2$. The capacitor at node $\mathrm{C}$ is controlled like the cap at B in phase $\Phi_{1}$. Next, when the clock $\Phi_{3}$ $=1$ the voltage at B and $\mathrm{C}$ would attain a level of $V_{r e f}\left(b_{1+k} / 2+b_{2+k}\right) / 2$. Similarly, when $\Phi_{1}=1$ again, the voltages at $\mathrm{C}$ and $\mathrm{D}$ will be equal to $V_{r e f}\left(b_{1+k} / 4+b_{2+k} / 2+b_{3+k}\right) / 2$. This is in fact, the output voltage of the first SC segment and the same process will continue for the following segments.

When all the parasitic capacitances of the circuit are neglected the output voltage of the SC pipeline DAC is linearly dependent on the values of input data according to

$$
V_{\text {out }}=V_{\text {ref }} \sum_{i=0}^{n-1} \frac{b_{i}}{2^{n}} 2^{i}
$$




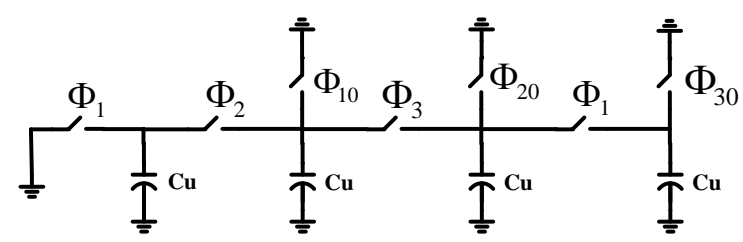

Figure 5.3: Model of first section of SC pipeline.

\subsection{Capacitive pipeline Array Analysis}

In order to choose the right values of unit capacitance and the sizes of switches, the analyses of noise, capacitor mismatch, settling time and clock feed-through effect are necessary.

\subsubsection{Nosie analysis}

Noise contribution from all switches to the output can be estimated using the superposition principle. Basically the hold portion of noise is considered since the track noise is not transferred except for two switches in the last section of the pipeline. For switch $\Phi_{1}$ of the first section (Fig. 5.3) the voltage transfer function to the output is $1 / 2^{n}$, where $n$ is the number of capacitors (DAC bits) in the pipeline. Its noise power spectral density (PSD) is subject to folding with a factor $1 /\left(2 R_{o n} C_{u} f_{s}\right)$ [98] due to the equivalent noise bandwidth $1 /\left(4 R_{o n} C_{u}\right)$ and switching frequency $f_{s}$, where $R_{o n}$ denotes the switch on-resistance.

Moreover, we observe that the transferred noise voltage remains on the output capacitor only for $2 / 3$ part of the clock period and otherwise the capacitor is discharged. The latter effect is reflected in the frequency domain by a sinc function shaping the noise spectrum accordingly. Hence, the respective noise PSD contribution (one sided) to the output at low frequency can be found from

$$
S_{1}(f)=4 k T R_{o n} \times \frac{1}{2 R_{o n} C_{u} f_{s}} \times \frac{1}{2^{2 n}} \times\left(\frac{2}{3}\right)^{2}
$$

The contribution of switch $\Phi_{10}$ is the same as of $\Phi_{1}$. For noise of the $\Phi_{2}$ switch we find the incident capacitance to be $C_{u} / 2$ and the respective held voltage to be shared by two $C_{u}$ capacitors in series. The transfer function to the output is $1 / 2^{n-1}$ so the noise contribution of $\Phi_{2}$ switch appears as

$$
S_{2}(f)=4 k T R_{o n} \times \frac{1}{R_{o n} C_{u} f_{s}} \times \frac{1}{2^{2}} \times \frac{1}{2^{2(n-1)}} \times\left(\frac{2}{3}\right)^{2}
$$




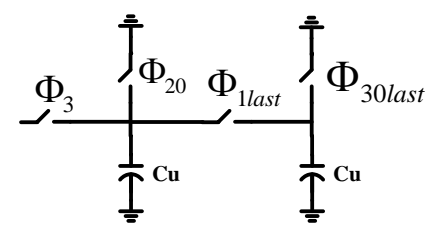

Figure 5.4: Model of last section of SC pipeline.

For switch $\Phi_{3}$ only the transfer function is changed to $1 / 2^{n-2}$ so $S_{3}(f)=S_{2}(f) / 4$.

For the last two switches in the pipeline (Fig. 5.4) also their track noise should be taken into account

$$
\begin{aligned}
& S_{\text {last }}(f)=4 k T R_{o n} \times \frac{1}{R_{s w} C_{u} f_{s}} \times \frac{1}{2^{2}} \times\left(\frac{1}{3}\right)^{2}+4 k T R_{o n} \times \frac{1}{2^{2}} \times \frac{1}{3} \\
& S_{3 l a s t}(f)=8 k T R_{o n} \times \frac{1}{4 R_{s w} C_{u} f_{s}} \times \frac{1}{2^{2}} \times\left(\frac{2}{3}\right)^{2}+8 k T R_{o n} \times \frac{1}{3}
\end{aligned}
$$

where for $\Phi_{1 \text { last }}$ contribution both the hold and track time is $1 /\left(3 f_{s}\right)$. For $\Phi_{\text {3last }}$ a resistance of $2 R_{o n}$ is assumed (two switches in series). The total hold noise by the floating switches $\Phi_{2}, \Phi_{3}, \Phi_{1}, \ldots$ can be estimated as

$$
S_{f}(f)=\frac{4 k T}{9 C_{u} f_{s}} \times\left(\sum_{k=1}^{n-1} \frac{1}{2^{2 k}}+\frac{1}{4}\right) \cong \frac{7 k T}{27 C_{u} f_{s}}
$$

and the hold noise contribution by the remaining switches (to ground or reference source) is

$$
S_{g}(f)=\frac{8 k T}{9 C_{u} f_{s}} \times\left(\sum_{k=1}^{n} \frac{1}{2^{2 k}}+\frac{1}{4}+\frac{1}{2^{2 n}}\right) \cong \frac{14 k T}{27 C_{u} f_{s}}
$$

The total noise PSD at the output including the track noise can be found from

$$
S_{\text {tot }}(f) \cong \frac{7 k T}{9 C_{u} f_{s}}+3 k T R_{o n}
$$

For practical design values the hold noise (kT/C) in (5.7) matters while the track noise term in can be omitted. 


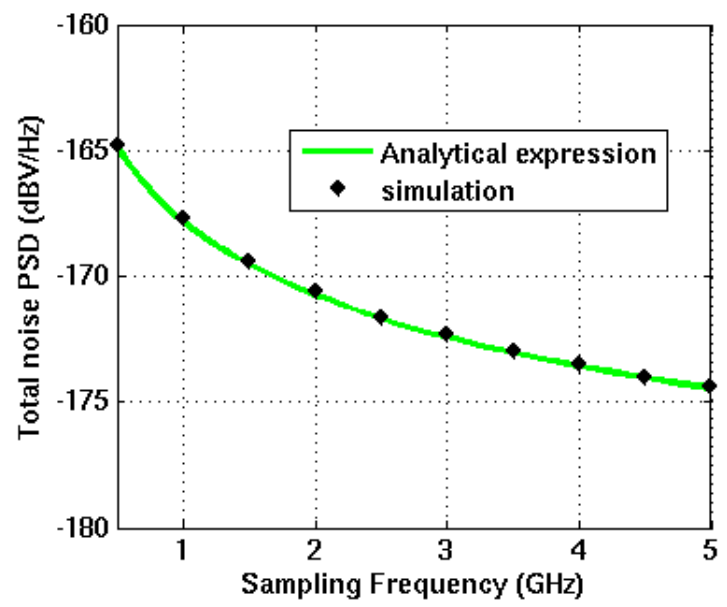

Figure 5.5: SC noise PSD comparison of analytical model (5.7) and SpectreRF ${ }^{\mathrm{TM}}$ simulations with $C_{u}=200 \mathrm{fF}, R_{o n}=30 \Omega$.

Fig. 5.5 shows a comparison of SC noise PSD obtained with (5.7) and by a circuit simulation model using SpectreRF ${ }^{\mathrm{TM}}$. The results are achieved by Transient noise simulation followed by spectrum averaging at low frequencies. Using Pnoise analysis the same result is obtained.

Generally, the SC noise should be less than quantization noise of DAC which can be calculated from

$$
S_{Q}(f)=\frac{V_{F S}^{2}}{12\left(2^{n}-1\right)^{2} B W}
$$

where $V_{F S}$ is the full-scale voltage, $n$ is the number of bits, and $B W=f_{s} / 2$. Then using (5.7) we can write

$$
\frac{7}{9} \frac{k T}{C_{u} f_{s}}<\frac{V_{F S}^{2}}{12 B W\left(2^{n}-1\right)^{2}}
$$

that can be seen as a design constraint for the unit capacitance $C_{u}$

$$
C_{u}>\frac{4.67 k T\left(2^{n}-1\right)^{2}}{V_{F S}^{2}}
$$

For example, for $n=12$ and $V_{F S}=1.3 \mathrm{~V}$, and $T=290 \mathrm{~K}$ from (5.10) we obtain $C_{u}>191$ $\mathrm{fF}$. Observe, that for the minimum value of $C_{u}$ the total noise level of DAC gets doubled and SNR is less by $3 \mathrm{~dB}$ compared to SNQR. 


\subsubsection{Capacitor mismatch analysis}

Following the analysis in [99] a similar estimate for voltage error due to capacitor mismatch can be attained

$$
V_{D N L} \approx \frac{\sqrt{2^{n}-1}}{2^{n}} \frac{\Delta C_{u}}{C_{u}} V_{F S}
$$

where $V_{F S}$ is the full-scale output range and $V_{D N L}$ is the differential nonlinearity of DAC. The standard deviation $\sigma\left(\Delta C_{u} / C_{u}\right)$ of the capacitor mismatch for a typical metalinsulator-metal (MIM) capacitor can be found from [102]

$$
\sigma\left(\frac{\Delta C_{u}}{C_{u}}\right)=\frac{K_{\sigma}}{\sqrt{A}} \quad \text { and } \quad C_{u}=K_{C} A
$$

where $A$ is the capacitor areawhereas $K_{\sigma}$ (matching coefficient) and $K_{C}$ (capacitance density) are technology constants provided by the chip manufacturer. From (5.11) and (5.12), the standard deviation of the voltage error caused by capacitor mismatch is found as

$$
\sigma\left(V_{D N L}\right) \approx \frac{\sqrt{2^{n}-1}}{2^{n}} V_{F S} \sigma\left(\frac{\Delta C_{u}}{C_{u}}\right)
$$

For the DAC accuracy, it is common to maintain $3 \sigma\left(V_{D N L}\right)$ less than a half LSB. Hence, the lower bound for the unit capacitor due to the mismatch in $n$-bit DAC is

$$
C_{u}>9 K_{C} K_{\sigma}^{2}\left(2^{n}-1\right)
$$

For $65 \mathrm{~nm}$ CMOS process we have $K_{\sigma}=0.5 \% \mu \mathrm{m}, K_{C}=5 \mathrm{fF} / \mu \mathrm{m}^{2}$ that for 12-bit DAC give the unit capacitor $C_{u}>5 \mathrm{fF}$. As already shown, the limit due to $\mathrm{kT} / \mathrm{C}$ noise calculated from (5.10) for $V_{F S}=1.3 \mathrm{~V}$ is $C_{u}>191 \mathrm{fF}$ so it largely prevails while the limit due to the capacitor mismatch can be neglected.

To verify the estimates by simulation, we refer to more accurate equations from [99]

$$
V_{D N L}=\frac{C_{u}+\left(\sqrt{2^{n-1}}-\sqrt{2^{n-1}-1}\right) \Delta C_{u}}{2^{n} C_{u}+\sqrt{2^{n}} \Delta C_{u}} V_{F S}-\frac{V_{F S}}{2^{n}}
$$

The standard variation of $V_{D N L}$ would be

$$
\sigma\left(V_{D N L}\right)=\frac{1+\left(\sqrt{2^{n-1}}-\sqrt{2^{n-1}-1}\right) \sigma\left(\frac{\Delta C_{u}}{C_{u}}\right)}{2^{n}+\sqrt{2^{n}} \sigma\left(\frac{\Delta C_{u}}{C_{u}}\right)} V_{F S}-\frac{V_{F S}}{2^{n}}
$$

From (5.12), we have 


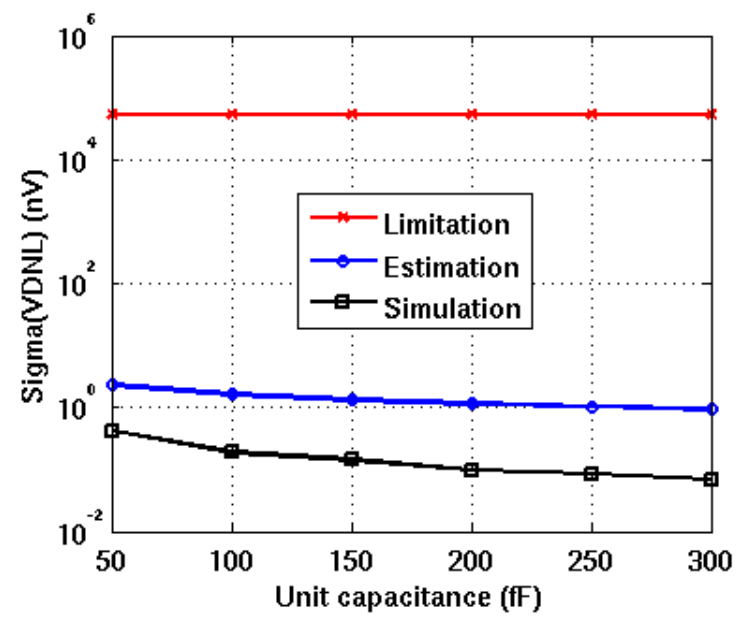

Figure 5.6: $\mathrm{V}_{\mathrm{DNL}}$ standard deviation comparison of estimated model (5.18) and SpectreRF ${ }^{\mathrm{TM}} \mathrm{MC}$ simulations against 1/6 LSB voltage of 12 bit DAC.

$$
\sigma\left(\frac{\Delta C_{u}}{C_{u}}\right)=\frac{K_{\sigma} \sqrt{K_{C}}}{\sqrt{C_{u}}}
$$

Substituting (5.17) to (5.16), we have

$$
\sigma\left(V_{D N L}\right)=\frac{1+\left(\sqrt{2^{n-1}}-\sqrt{2^{n-1}-1}\right) \frac{K_{\sigma} \sqrt{K_{C}}}{\sqrt{C_{u}}}}{2^{n}+\sqrt{2^{n}} \frac{K_{\sigma} \sqrt{K_{C}}}{\sqrt{C_{u}}}} V_{F S}-\frac{V_{F S}}{2^{n}}
$$

With $65 \mathrm{~nm}$ CMOS process of STM foundry we have $K_{\sigma}=0.5 \% \mu \mathrm{m}, K_{C}=5 \mathrm{fF} / \mu \mathrm{m}^{2}$. Fig. 5.6 gives a comparison of $\mathrm{V}_{\mathrm{DNL}}$ standard deviation obtained with model (5.18) and SpectreRF ${ }^{\mathrm{TM}}$ simulations. The simulation results are achieved by statistical Monte-Carlo analysis only with the focus on capacitor variation whereas the switches are assumed ideal and the simulation count is 100 . A very low clock frequency was used to verify the static $\mathrm{V}_{\mathrm{DNL}}$ with input data of MSB transition [99]. We usually use $3 \sigma$ standard, meaning that $3 \sigma\left(V_{D N L}\right)$ should be less than a half of $\mathrm{V}_{\mathrm{LSB}}$. The limitation line shown in Fig. 5.6 is therefore one sixth of $\mathrm{V}_{\mathrm{LSB}}$ of 12-bit resolution DAC which is $53 \mu \mathrm{V}$ with $V_{F S}$ $=1.3 \mathrm{~V}$. The $\mathrm{V}_{\mathrm{DNL}}$ standard deviations are in nano-volt range while those from simulation are by one order of magnitude less than this. The results from simulation are lower than the ones from estimation because in calculation we assumed total variation of mismatch as maximum [99] while the variation of unit capacitors mismatch in 
Monte-Carlo simulation is randomly distributed. Importantly, the both values are by orders of magnitude less than the limitation voltage of 12-bit DAC. Therefore the limit due to the capacitor mismatch can be neglected while the one due to $\mathrm{kT} / \mathrm{C}$ noise prevails in this case.

\subsubsection{Clock feed-through effect}

To discuss the feed-through effect we consider three groups of switches in the SC array (Fig. 5.2), namely: data switches, charge/discharge switches, and charge redistribution switches. The clock feed-through caused by the data switches is not meaningful because there is always a low time-constant path either to $V_{\text {ref }}$ or GND. Unlike this, the feed-through related to the charge switches changes the capacitor voltage when they are switched off because the respective capacitors see high impedance during this period of time. Since the switches are designed as transmission gates, there is inherent partial cancelation of this effect due to the rising and falling edge applied at the same time to the PMOS and NMOS device, respectively. This cancelation requires correction by dummy transistors [97] since the PMOS devices are three times larger than the NMOS (for balance of resistance).

The charge redistribution switches require compensation of the feed-through effect not only upon switching off but also switching on, taking thereby more effect on the transfer function and the DAC linearity. For this reason the redistribution switches require more precise feed-through cancelation that also entails an extra design constraint. Assuming the feed-through voltage to be limited by a half LSB we have

$$
K_{d i f} K_{\text {comp }} \frac{C_{P}(W)}{C_{u}+C_{P}(W)} V_{d d}<\frac{V_{r e f}}{2^{n+1}}
$$

where $C_{p}(W)$ is parasitic capacitance of a switch with respect to the switch size, while $K_{\text {comp }}$ is a constant representing the feed-through compensation by the dummy devices. This constant can be identified during optimization of the charge redistribution switches. Assuming that the total mismatch between two single-ended branches of differential topology (including mismatch of switches, caps, jitter, skew) is $K_{d i}$, from (5.19) the limit for $C_{u}$ follows

$$
C_{u}>\left(K_{d i f} K_{c o m p} 2^{n+1} \frac{V_{d d}}{V_{r e f}}-1\right) C_{P}(W)
$$

\subsubsection{Settling time analysis}

Due to imperfections of the transistor switches ( $R_{\text {off }} / R_{\text {on }}$ resistances) the leakage and incomplete settling in the capacitor array result in voltage errors which degrade DAC linearity [96]. Using the schematic in Fig. 5.2, we can consider an error at node B for $\Phi_{1}$ $=0$ while $\Phi_{2}=1$. For the incident bit value equal to 1 , there is a leakage current from $V_{\text {ref }}$ through $R_{\text {off }}$ to the capacitance $2 C_{u}$ seen from node B. In this case, the on-resistance 


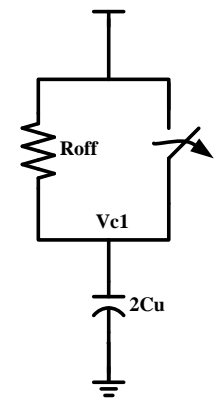

(a)

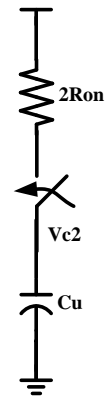

(b)

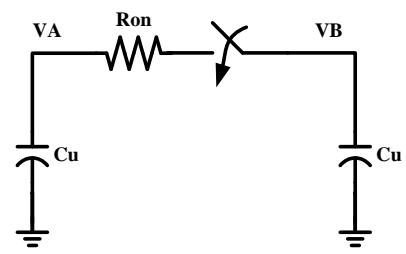

(c)

Figure 5.7: Charged and discharged circuits: (a) Leakage due to off-resistance of data switches, (b) Incomplete charge due to on-resistance of data switches (c) Incomplete charge redistribution due to inbetween switches.

of switch $\Phi_{2}$ can be neglected since $R_{o n} \ll R_{o f f}$. The respective voltage change (Fig. 5.7a) can be expressed as

$$
V_{C 1}=V_{r e f}\left(1-e^{\frac{-t_{\text {pulse }}}{2 R_{o f f} C_{u}}}\right)
$$

where $R_{\text {off }}$ is the off-resistance of a charge switch, $T_{s}=1 / f_{s}$ is clock period, and $t_{\text {pulse }}$ is the pulse width. Importantly, $t_{\text {pulse }}<1 /\left(3 f_{s}\right)$ to guarantee non-overlapping 3-phase clock operation. In order to keep the voltage error (21) less than half LSB we find the lower bound of $R_{\text {off }}$

$$
R_{o f f}>-\frac{t_{p u l s e}}{2 C_{u}} \frac{1}{\ln \left(1-2^{-n-1}\right)}
$$

Similarly, we can find the upper bound of the switch on-resistance. Fig. 5.7b shows the charge phase with incident input bit equal to 1 . The respective settling time error can be found from

$$
V_{C 2}=V_{r e f} e^{\frac{-t_{\text {pusse }}}{2 R_{\text {on }} C_{u}}}
$$

This error should also be limited by half LSB that leads to

$$
R_{\text {on }}<\frac{t_{\text {pulse }}}{2 C_{u}} \frac{1}{\ln \left(2^{n+1}\right)}
$$

For completeness, we should also consider an error during charge redistribution phase shown in Fig. 5.7c. To calculate $V_{B}$, we use superposition 


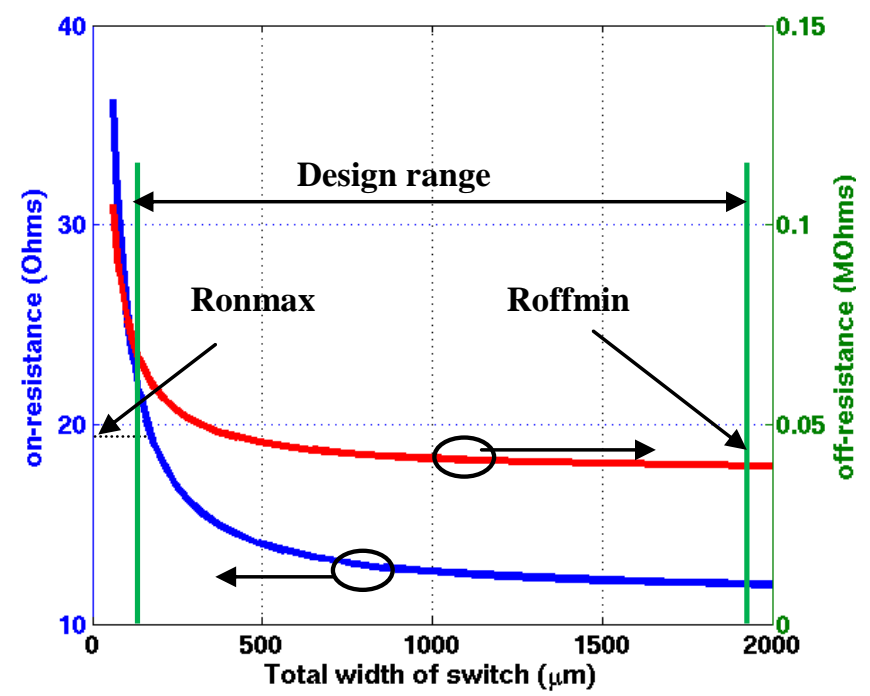

Figure 5.8: Off-resistance and on-resistance of T-gate switch vs. width in 65nm CMOS with $L=0.06 \mu \mathrm{m}\left(W_{p} / W_{n}=3\right)$.

$$
V_{B}=\frac{V_{A 0}}{2}\left(1-e^{\frac{-t_{\text {puse }}}{T}}\right)-\frac{V_{B 0}}{2}\left(1-e^{\frac{-t_{\text {puse }}}{T}}\right)+V_{B 0}
$$

where $V_{A 0}$ and $V_{B 0}$ are initial values of $V_{A}$ and $V_{B}$, respectively. The time constant $T$ $=R_{o n} C_{u} / 2$. In the worst case, for $V_{A 0}=0$ and $V_{B 0}=V_{\text {ref }}$ this voltage will be

$$
V_{B}=\frac{V_{r e f}}{2}\left(1+e^{\frac{-2 t_{\text {pulse }}}{R_{\text {on }} C_{u}}}\right)
$$

As the error voltage $\left(V_{B^{-}} V_{\text {ref }} / 2\right)$ should be less than half LSB

$$
R_{\text {on }}<\frac{1}{\ln \left(2^{n-1}\right)} \frac{2 t_{\text {pulse }}}{C_{u}}
$$

Since (5.27) is more relaxed compared to (5.24) the upper bound for $R_{o n}$ is set by (5.24).

The settling conditions (5.22) and (5.24) appear stringent for high frequency clock. For illustration, let us consider $f_{s}=3 \mathrm{GHz}, t_{\text {pulse }}=50 \mathrm{ps},\left(t_{\text {pulse }}<1 /\left(3 f_{s}\right)\right), n=7$, and $C_{u}$ $=200 \mathrm{fF}$. In this case the lower bound of $R_{\text {off }}=30 \mathrm{k} \Omega$ and the higher bound of $R_{o n}=19.5 \Omega$. A suitable size of a switch (transmission gate) in $65 \mathrm{~nm}$ CMOS with channel length $L=0.06 \mu \mathrm{m}$ is depicted in Fig. 5.8. The width range of $\left[W_{\min }, W_{\max }\right]=$ $[150,2000] \mu \mathrm{m}$ satisfies the the design constraints (5.22) and (5.24). However, for higher DAC resolutions the available design range is reduced and already for $n>8$, it will vanish unless the demand of half LSB accuracy is relaxed. 


\subsubsection{Bounds for unit capacitor}

Having mapped the resistances $R_{o f f}$ and $R_{o n}$ onto the width of the switching devices, we can identify functions $R_{\text {off }}(W)$ and $R_{o n}(W)$, and redefine our design task by combing (5.22) and (5.24) as follows

$$
-\frac{t_{\text {puse }}}{2 R_{\text {off }}(W)} \frac{1}{\ln \left(1-2^{-n-1}\right)}<C_{u}<\frac{t_{\text {pulse }}}{2 R_{\text {on }}(W)} \frac{1}{\ln \left(2^{n+1}\right)}
$$

In this case, the lower- and upper bound for $C_{u}$ are defined in terms of the switch size $W$, pulse time $t_{\text {pulse }}$, and the number of bits $n$. In fact, also $t_{\text {pulse }}$ depends on $W$ due to parasitic capacitance of a switch and the driving source resistance that reduce the pulse time. Estimating the rise/fall time $t_{r f}$ from $10 \%$ to $90 \%$ voltage change we find

$$
t_{\text {rf }}=\ln (9) R_{d} C_{g}(W)
$$

where $R_{d}$ is the driver resistance and $C_{g}(W)$ is the gate capacitance with respect to the switch width $W$. Then $t_{\text {pulse }}$ time appears as

$$
t_{\text {pulse }}=\frac{1}{3 f_{S}}-t_{n}-4 \ln (9) R_{d} C_{g}(W)
$$

where $t_{n}$ is a latency time to guarantee non-overlapping clock. The additional reduction by $4 \times t_{r f}$ is due to the rising and falling time, while $C_{g}$ value is doubled due to a dummy switch (to compensate for the feed-through effect [97]). Based on this (5.28) can be rewritten as

$$
\frac{\left(3 f_{S}\right)^{-1}-t_{n}-8.8 R_{d} C_{g}(W)}{-2 \ln \left(1-2^{-n-1}\right) R_{o f f}(W)}<C_{u}<\frac{\left(3 f_{S}\right)^{-1}-t_{n}-8.8 R_{d} C_{g}(W)}{2 \ln \left(2^{n+1}\right) R_{o n}(W)}
$$

This result is illustrated in Fig. 5.9 for clock frequency $f_{s}=3 \mathrm{GHz}, t_{n}=30 \mathrm{ps}, n=7$ with solid lines representing the upper and lower bounds for $C_{u}$. The design area $W-C_{u}$ is additionally limited by the feed-through condition (5.20) shown as the straight line (green) lower bound. The conditions due to $\mathrm{kT} / \mathrm{C}$ noise and capacitor mismatch are negligible in this case. For $W<570 \mu \mathrm{m}$ the $C_{u}$ lower bound due to $R_{\text {off }}$ applies. For $W>$ $570 \mu \mathrm{m}$ and $W<780 \mu \mathrm{m}$ the lower bound due to the feed-through limit applies.

The dashed lines illustrate the design area for the 8-bit resolution to vanish (the upper bound goes below the lower bound). If the accuracy requirement of half LSB is relaxed the design area can be restored. Interestingly, for 1-LSB accuracy and $n$-bit resolution the same design area is achieved as for (n-1)-bit with half-LSB accuracy, so the design area shown in Fig. 5.9 for the 7-bit DAC is also valid for the 8-bit provided the accuracy constraints are changed to 1-LSB. 


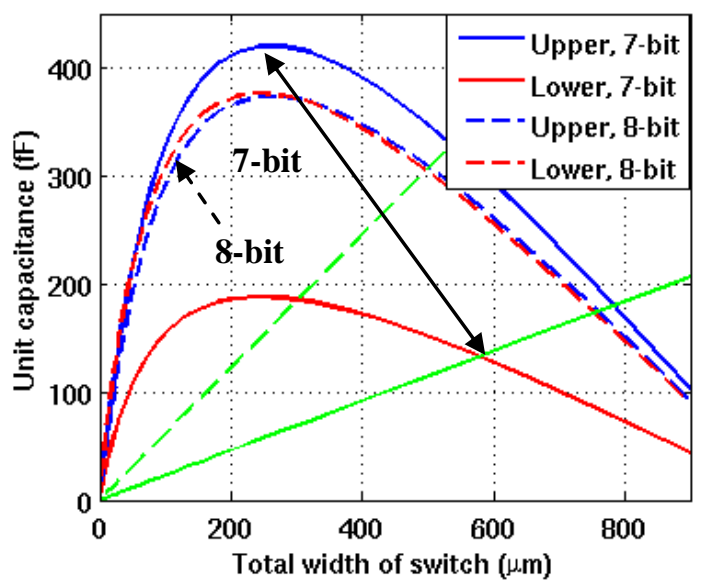

Figure 5.9: Design area W-C $\mathrm{C}_{\mathrm{u}}$ for 7-bit and 8-bit SC DAC, $f_{S}=3 \mathrm{GHz}, K_{\text {dif }}=20 \%$ and $K_{\text {comp }}=1 \%$.

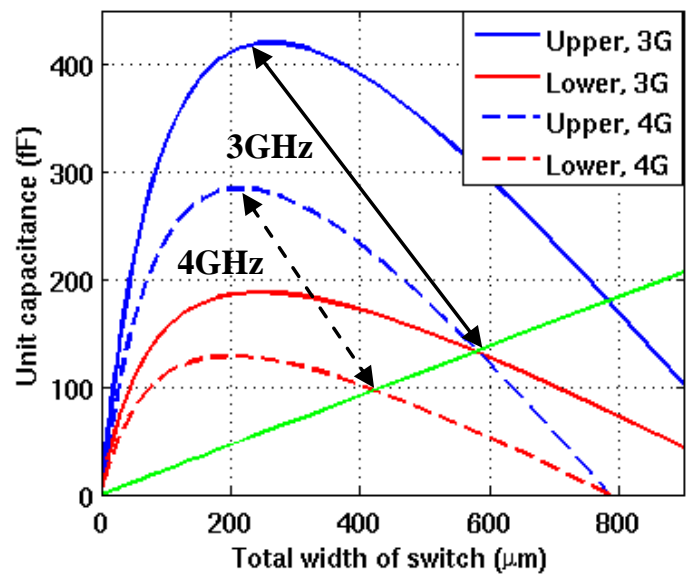

Figure 5.10: Design area $\mathrm{W}-\mathrm{C}_{\mathrm{u}}$ for 7-bit SC DAC with different clock frequency $\left(f_{s}\right)$ $\left(K_{\text {dif }}=20 \%, K_{\text {comp }}=1 \%\right)$.

According to (5.31) the design area largely shrinks with the clock frequency as well. This is illustrated in Fig. 5.10 when the clock frequency is increased from $3 \mathrm{GHz}$ to 4 $\mathrm{GHz}$ for $n=7$. In this case the feed-through bound is not changed but the other two bounds due to (5.31), go down largely reducing the design area.

To increase the speed of this SC DAC, we have to reduce its resolution. For example, increasing the clock frequency to $f_{s}=12 \mathrm{GHz}$ a convenient design area for a 


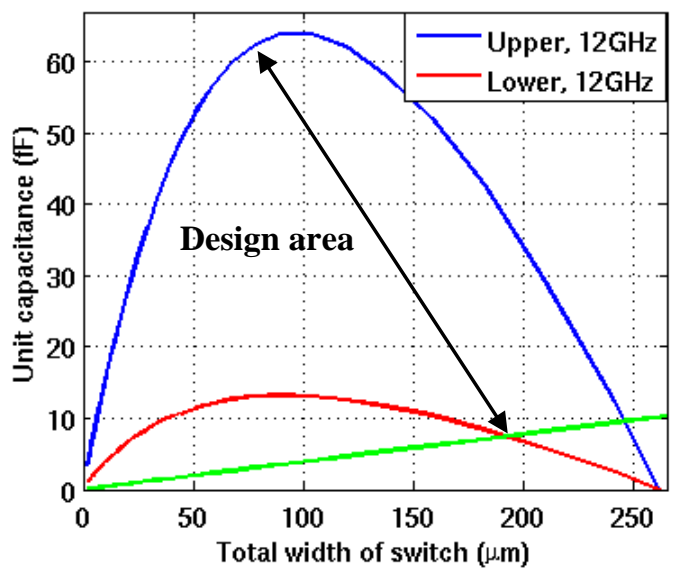

Figure 5.11: Design area $\mathrm{W}-\mathrm{C}_{\mathrm{u}}$ for 6-bit SC DAC at $12 \mathrm{GHz}$ clock frequency.

6-bit DAC is available as shown in Fig. 5.11. Then, with the corresponding Nyquist bandwidth $<6 \mathrm{GHz}$ the estimated maximum data rate amounts for $72 \mathrm{Gbps}$.

In the analysis and simulations, the reference voltage was assumed as an ideal source. However, previous work such as [87] has shown that the reference voltage can for example be generated using on-chip low drop-out regulator (LDO).

In principle, the tradeoff between the resolution and bandwidth (or data rate) in this design can be conveniently moved towards higher levels of those quantities by making use of newer CMOS technologies. For illustration in Appendix-E we demonstrate the DAC design areas for Fully Depleted Silicon-on-Insulator (FD SOI) $28 \mathrm{~nm}$ CMOS process that enables a significant increase of the resolution and bandwidth. Clearly, the tradeoff between them still exists. However, other features such as better element matching, compared to current steering solutions, make SC DACs even more appealing.

\subsection{Output Driver Design}

\subsubsection{Problem and solution}

The output voltage of high speed DACs for wireless and wire LAN communications can also exceed $300 \mathrm{mV}_{\mathrm{pp}}$. In practice, we have to treat this voltage as a large signal like in the power amplifier case. There are several linearization techniques well established in power amplifier design [89]. Pre-distortion as one of them was already applied in a high speed DAC to compensate for non-linearity of its output driver [62]. Using the main and auxiliary amplifier/driver for non-linearity cancellation [90, 91] is another 


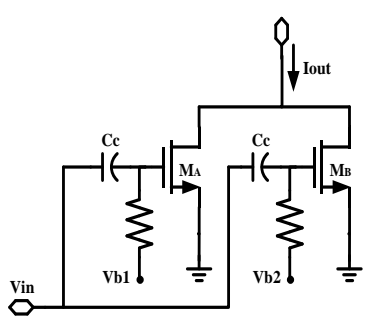

(a)

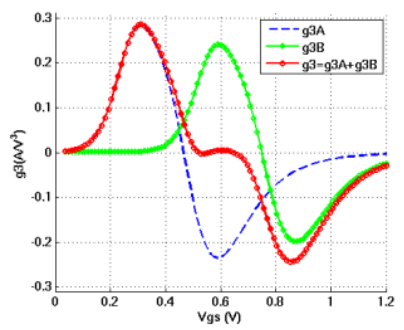

(b)

Figure 5.12: (a) DS technique with dual-NMOS. (b) Third-order distorion terms of the main transistor $\left(\mathrm{g}_{3 \mathrm{~A}}\right)$, auxiliary transistor $\left(\mathrm{g}_{3 \mathrm{~B}}\right)$, and total output $\left(\mathrm{g}_{3}\right)$ using ST $65 \mathrm{~nm}$ CMOS process, $(W / L)_{\mathrm{MA}}=20 / 0.065 \mu \mathrm{m},(W / L)_{\mathrm{MB}}=17 / 0.065 \mu \mathrm{m}, V_{d s}=1.2 \mathrm{~V}$ and $V_{\text {shift }}=282 \mathrm{mV}$.

possible solution. As an additional inspiration the techniques developed for low noise amplifiers (LNAs) can also be considered [18, 20, 92, 93, 95]. In particular, the thirdorder distortions can be cancelled using various linearization techniques, like negative feedback, harmonic termination, optimum biasing, feed-forward, or derivative superposition (DS).

In this design, we are targeting high SFDR and bandwidth (>1 GHz). This makes the driver design a challenge. Fig. 5.12 (a) shows a circuit suitable for third-order nonlinearity cancellation by DS method that has been recognized both in small- and large signal applications such as LNAs [20] and power amplifiers [94], respectively. This technique is based on cancellation of the transconductance $g_{3}\left(\mathrm{~d}^{3} I_{d} / \mathrm{d}\left(V_{g s}\right)^{3}\right)$ of the main transistor and $g_{3}$ of the auxiliary one for properly biased devices.

As shown in Fig. 5.12 (b), if $V_{g s}$ is biased around [0.52-0.67] $\mathrm{V}$ the output current has negligible $3^{\text {rd }}$ order transconductance. So the suitable input swing is about $150 \mathrm{mV}_{\mathrm{pp}}$ that is however, not sufficient for most applications. Moreover, the auxiliary transistor has to be biased in weak inversion to generate positive $g_{3 \mathrm{~B}}$ value [18]. Larger input swing tend to push this transistor into the off region for a part of the input cycle raising the distortion. With $300 \mathrm{mV}_{\mathrm{pp}}$ swing this circuit cannot provide HD3 better than $-50 \mathrm{~dB}$.

To overcome these problems, we propose a combination of a simple inherent feedback and DS technique that is illustrated in Fig. 5.13. The negative feedback technique helps to reduce both second- and third order non-linearity of a system [18]. Specifically, we try to avoid using inductors and complexity by complementary circuits necessary in different solutions.

Like for the inductive source-degeneration [18], in this case $A_{I I P 3}$ improves approximately by a ratio $\left(1+g_{m 1} R_{S}\right)^{3 / 2}$. Moreover, the source resistor provides wideband matching for the driver load.

A large value of $g_{m 1} R_{S}$ is also useful to maximize the closed-loop voltage gain of the driver, ideally approaching one. Since the effective $R_{S}$ value is limited by the $50 \Omega$ load, 


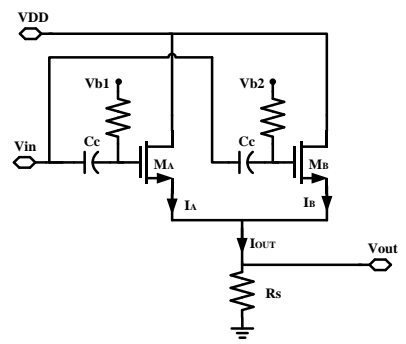

(a)

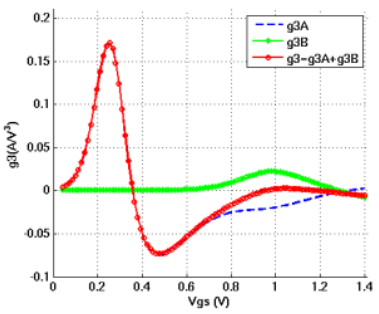

(b)

Figure 5.13: (a) DS technique with resistive feedback. (b) Third-order distorion terms of the main transistor $(\mathrm{g} 3 \mathrm{~A})$, auxiliary transistor $\left(\mathrm{g}_{3 \mathrm{~B}}\right)$, and total output $\left(\mathrm{g}_{3}\right)$ using ST 65nm CMOS process, $(W / L)_{\mathrm{MA}}=20 / 0.065 \mu \mathrm{m},(W / L)_{\mathrm{MB}}=17 / 0.065 \mu \mathrm{m}, V_{d s}=1.4 \mathrm{~V}$ and $V_{\text {shift }}=452 \mathrm{mV}$.

more gain can only be achieved by increasing $g_{m 1}$ at the expense of the power consumption.

Fig. 5.13 (a) shows a half of the proposed output driver designed as a differential circuit. The voltage at the output of SC pipeline can be large enough to provide around $300 \mathrm{mV}_{\mathrm{pp}}$ at the driver output even for a moderate gain $g_{m 1} R_{S}$. Since we use $1.2 \mathrm{~V}$ supply to limit power consumption of the driver, not much voltage headroom is left to keep the signal within a linear region of the input/output characteristics. Inevitably, the signal is exposed to non-linear behavior of the transistors and hence, good enough cancelling of the non-linear effects is necessary.

As shown in Fig. 5.13 (b), the cancelling range is much wider compared to the previous case. The both transistors operate in saturation that makes the circuit less sensitive to large input swing. Also larger bias voltages are allowed providing larger transconductance values $g_{m 1}$ and lower HD3 distortions at the same time.

\subsubsection{Volterra series model}

The advantage of the DS technique combined with the resistive feedback will be discussed using the Volterra series model [101]. We focus on the third-order distortions as they used to prevail over the other distortions

$$
H D 3=\frac{1}{4} \frac{\left|G_{3}\left(j \omega_{1}, j \omega_{1}, j \omega_{1}\right)\right|}{\left|G_{1}\left(j \omega_{1}\right)\right|} A^{2}
$$

where $A$ stands for the amplitude of a sinusoidal signal at the driver input. Using the transfer functions (A.5.11) and (A.5.13) derived in Appendix-C, HD3 of the proposed driver can be estimated by 


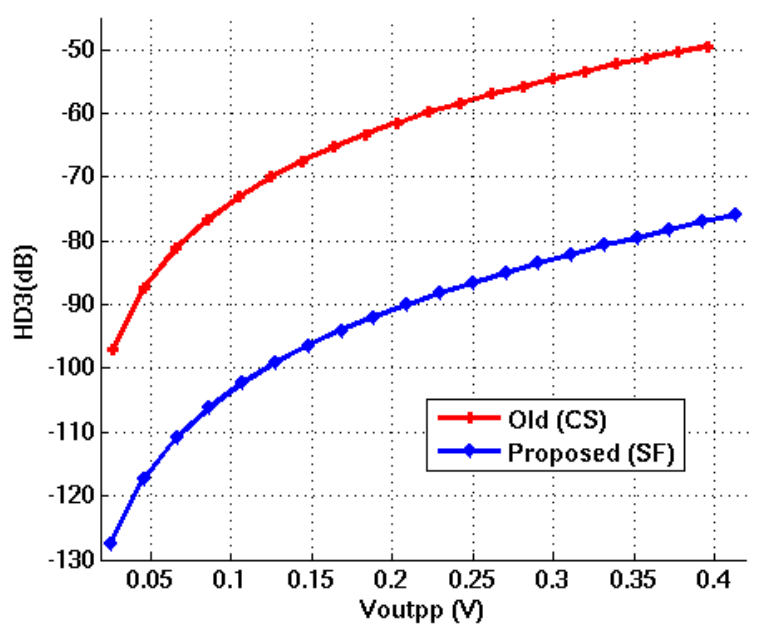

Figure 5.14: Comparison of conventional DS technique and DS technique combined with resistive feedback.

$$
\begin{aligned}
& H D 3_{S F}=\frac{1}{4} \frac{A^{2}}{g_{1 A}+g_{1 B}} \times \\
& \frac{\left(g_{3 A}+g_{3 B}\right)}{\left[1+R_{S}\left(g_{1 A}+g_{1 B}\right)\right]^{3}}-\frac{2 R_{S}\left(g_{2 A}+g_{2 B}\right)^{2}}{\left[1+R_{S}\left(g_{1 A}+g_{1 B}\right)\right]^{4}}
\end{aligned}
$$

Similarly, using (A.5.14) and (A.5.16), we can calculate HD3 of the DS-only driver

$$
H D 3_{C S}=\frac{1}{4} \frac{\left(g_{3 A}+g_{3 B}\right)}{g_{1 A}+g_{1 B}} A^{2}
$$

For the bias conditions of $V_{g s A}=1.2 \mathrm{~V}, V_{g s B}=0.75 \mathrm{~V}, V_{d d}=1.2 \mathrm{~V}, R s=50 \Omega$, and using the same transistor sizes for both circuits $\left((L / W)_{\mathrm{MA}}=(20 / 0.065)\right.$, $\left.(L / W)_{\mathrm{MB}}=(17 / 0.065)\right)$, the following transconductances are achieved: $g_{1 \mathrm{~A}}=19.6 \mathrm{mS}$, $g_{1 B}=5.2 \mathrm{mS}, g_{2 A}=1.6 \mathrm{mS}, g_{2 B}=44 \mathrm{mS}, g_{3 A}=-50 \mathrm{mS}, g_{3 B}=158.9 \mathrm{mS}$. The comparison of (33) and (34) with these values shows HD3 of the proposed circuit to be by $26.8 \mathrm{~dB}$ better than that of the DS-only circuit. This result is very close to the simulation result shown in Fig. 5.14. For $300 \mathrm{mV}_{\mathrm{pp}}$ output swing and $50 \Omega$ resistive load, HD3 $<-80 \mathrm{~dB}$ is attained that corresponds to 13-bit DAC resolution.

Within the same bias voltage range also the second-order distortion term can be partly cancelled. Using the Volterra model we have 


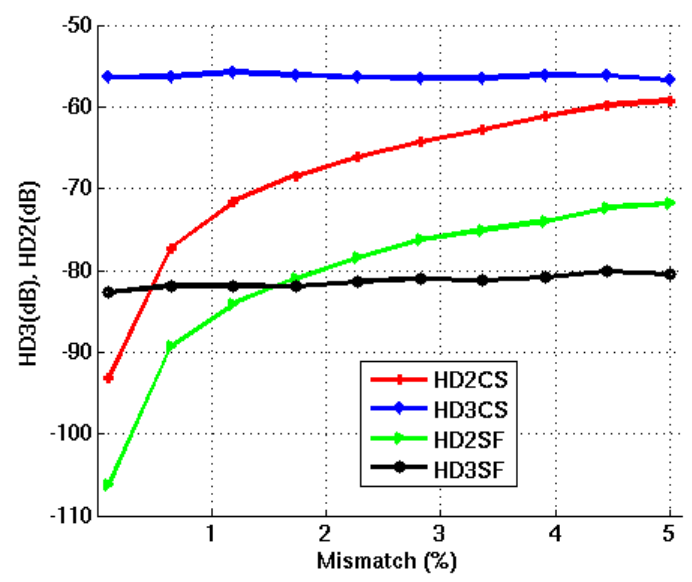

Figure 5.15: Comparison conventional (CS) and proposed DS technique with resistive load (SF) under mismatch.

$$
H D 2=\frac{1}{2} \frac{\left|G_{2}\left(j \omega_{1}, j \omega_{1}\right)\right|}{\left|G_{1}\left(j \omega_{1}\right)\right|} A
$$

and from (A.5.11) and (A.5.12)

$$
H D 2_{S F}=\frac{1}{2} \frac{\left(g_{2 A}+g_{2 B}\right)}{\left(g_{1 A}+g_{1 B}\right)} \frac{1}{\left[1+R_{S}\left(g_{1 A}+g_{1 B}\right)\right]^{2}} A
$$

Similarly, from (A.5.14) and (A.5.15)

$$
H D 2_{C S}=\frac{1}{2} \frac{\left(g_{2 A}+g_{2 B}\right)}{g_{1 A}+g_{1 B}} A
$$

By comparison of (5.36) and (5.37), the proposed driver shows HD2 by $15 \mathrm{~dB}$ better than its counterpart. This result is also verified by simulations presented in Fig. 5.15. As the driver is designed as a diffrential circuit a mismatch between its two branches was introduced to model HD2. Also HD3 proves immune to the mismatch.

For high frequency operation the driver circuit model would be refined as shown in Fig. 5.16. The parasitic resistances and capacitances $r_{o A}, r_{o B}, C_{d s A}$ and $C_{d s B}$ of the two transistors can be merged as $Z_{\text {out }}=\left(Z_{S}\left\|r_{o A}\right\| r_{o B}\left\|\left(1 / s C_{d s A}\right)\right\|\left(1 / s C_{d s B}\right)\right.$ with $Z_{S}=$ $\left(R_{S} \|\left(1 / s C_{\text {load }}\right)\right.$ and $C_{g s}=C_{g s A}+C_{g s B}$. Since $r_{o A}, r_{o B} \gg R_{S}$ and $C_{\text {load }} \gg C_{d s B}, C_{d s B}$, we can approximate the driver output impedance as $Z_{\text {out }}=\left(R_{S} \|\left(1 / s C_{\text {load }}\right)\right.$ 


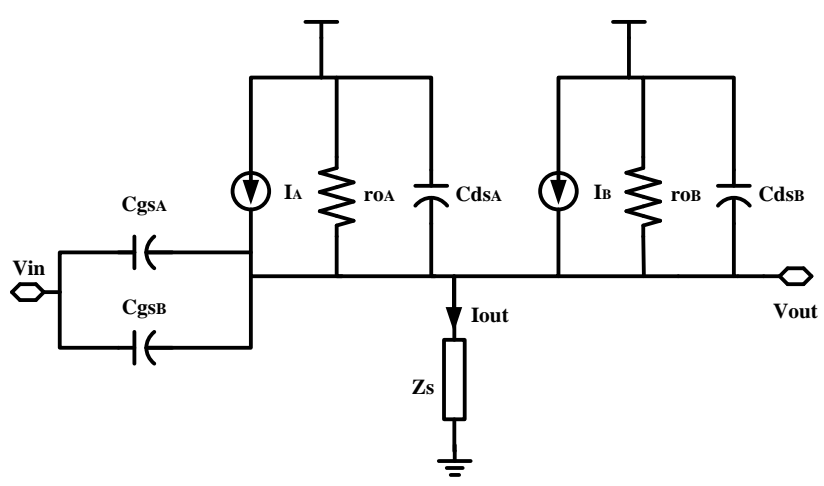

Figure 5.16: Equivalent circuit of the proposed linear output driver.

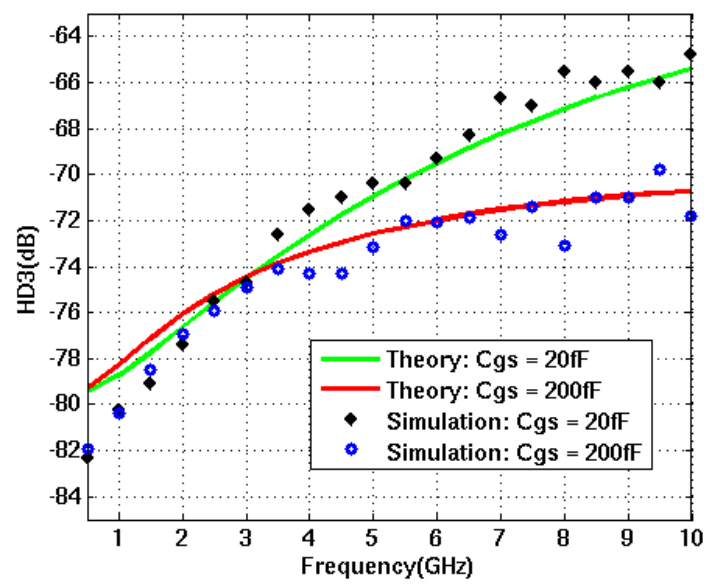

Figure 5.17: HD3 comparison of Volterra series model (5.38) and SpectreRF circuit simulations for proposed output driver.

Using (5.32) and (A.5.24), (A.5.26) of Appendix-D we can calculate HD3 of the output voltage in the proposed circuit

$$
\begin{aligned}
& H D 3=\frac{A^{2}}{4} \times \frac{1+Z_{\text {OUT }}\left(\omega_{1}\right)\left[g_{1 A}+g_{1 B}+j\left(\omega_{1}\right) C_{g s}\right]}{\mid 1+Z_{\text {OUT }}\left(\omega_{1}+\omega_{2}+\omega_{3}\right)\left[g_{1 A}+g_{1 B}+j\left(\omega_{1}+\omega_{2}+\omega_{3}\right) C_{g s}\right]} \times \\
& \left|\frac{Z_{\text {OUT }}\left(\omega_{1}+\omega_{2}+\omega_{3}\right)\left[\left(g_{3 A}+g_{3 B}\right)\left(1-G_{1}(\omega)\right)^{3}-2\left(g_{2 A}+g_{2 B}\right)\left[G_{2}\left(\omega_{1}+\omega_{2}\right)-\overline{G_{2}\left(\omega_{1}+\omega_{2}\right) G_{1}(\omega)}\right]\right]}{Z_{\text {OUT }}\left(\omega_{1}\right)\left[g_{1 A}+g_{1 B}+j\left(\omega_{1}\right) C_{g s}\right]}\right|
\end{aligned}
$$


HD3 obtained by the Volterra series model (5.38) and by SpectreRFTM simulations is depicted in Fig. 5.17 for two $C_{g s}$ values. HD3 tends to degrade with frequency beginning from the low cut-off frequency of $Z_{\text {OUT }}$. For higher frequencies the ratio $Z_{\text {OUT }}\left(j \omega_{1}, j \omega_{2}, j \omega_{3}\right) / Z_{\text {OUT }}\left(j \omega_{1}\right) \rightarrow 1 / 3$. Also the other numerator to denominator terms tend to settle and this effect is more pronounced for the larger $C_{g s}$ value, which provides better HD3 for higher frequencies at the expense of reduced voltage gain.

\subsubsection{Thermal noise analysis}

Using the schematic shown in Fig. 5.13, we can replace the transistors MA and MB by one device with transconductance $g_{m}=g_{m A}+g_{m B}$ and the corresponding input-referred voltage noise PSD of $4 k T \gamma / g_{m}$. By simple calculations the noise by the $R_{S}$ resistor can be found as $4 k T R_{S} /\left(g_{m} R_{S}\right)^{2}$ whereas the total input-referred noise PSD would be

$$
\overline{e_{n}^{2}}=4 k T\left(\frac{\gamma}{g_{m}}+\frac{1}{g_{m}^{2} R_{S}}+\frac{R_{B}}{2}\right)
$$

This can be compared to SC noise (5.7)

$$
\frac{7 k T}{9 C_{u} f_{S}}=4 k T\left(\frac{\gamma}{g_{m}}+\frac{1}{g_{m}^{2} R_{S}}+\frac{R_{B}}{2}\right)
$$

For example, using $C_{u}=200 \mathrm{fF}, f_{S}=3 \mathrm{GHz}, \gamma=1, g_{m}=40 \mathrm{mS}$, and $R_{S}=50 \Omega$, from (5.40) we find $R_{B} \approx 600 \Omega$ which can be considered the upper bound of the bias resistance.

\subsection{Simulation Results}

The circuit has been simulated using Cadence ${ }^{\mathrm{TM}}$ software with RF transistor models (65 nm CMOS). The driver output will be connected to an off-chip load (50 $\Omega$ ) and parasitic capacitances caused by wire-bonding cannot be avoided. In simulation we assume that parasitic capacitance equal to $100 \mathrm{fF}$. For signal frequencies up to $5 \mathrm{GHz}$ the gain drop remains within $0.5 \mathrm{~dB}$ shown in Fig. 5.18.

Using $1.2 \mathrm{~V}$, we distribute the supply current between the main and the auxiliary transistor to be $15.4 \mathrm{~mA}$ (providing $g_{1 \mathrm{~A}}=37.1 \mathrm{mS}$ ) and $1.2 \mathrm{~mA}$ (providing $g_{1 B}=2.9$ $\mathrm{mS}$ ), respectively. The total current through $R_{s}$ would be $16.6 \mathrm{~mA}$ with the total $g_{m}$ of 40 mS. With $R_{s}=65 \Omega$ and $R_{\text {load }}=50 \Omega$, the open-loop gain can be calculated as $\left(R_{\text {load }} \| R_{s}\right) \times\left(g_{1 A}+g_{1 B}\right)=1.13$ which results in the feedback-loop gain of $-5.5 \mathrm{~dB}$. 


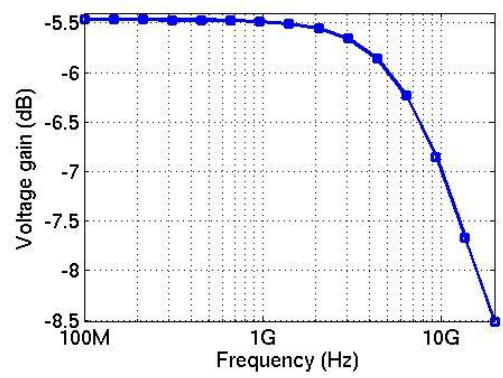

Figure 5.18: Voltage gain of output driver.

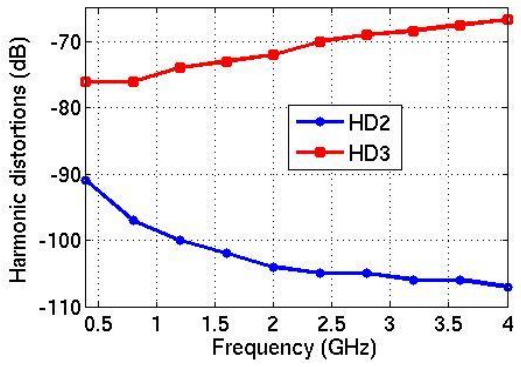

Figure 5.19: HD3 and HD2 versus frequency.

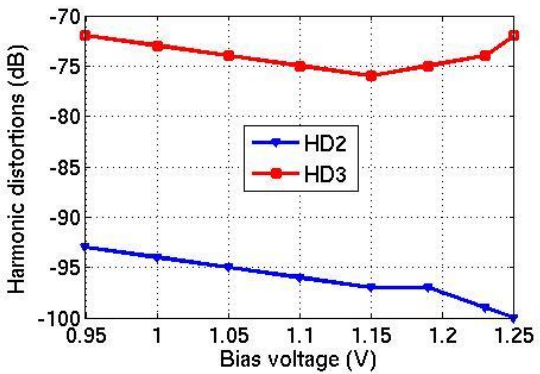

Figure 5.20: HD3, HD2 versus bias voltage.

Fig. 5.18 shows the voltage gain of the driver close to $-5.5 \mathrm{~dB}$ over the band of $0.1-5$ GHz. It changes only by $0.5 \mathrm{~dB}$ at $5 \mathrm{GHz}$ and this drop is mainly due to the intentionally added parasitic capacitance $100 \mathrm{fF}$. The corresponding cut-off frequency of the driver is approx. $20 \mathrm{GHz}$. Within the band of $0.5-4 \mathrm{GHz}, \mathrm{HD} 2<-90 \mathrm{~dB}$ and HD3 $<-66 \mathrm{~dB}$ are attained as shown in Fig. 5.19. The dependence of HD2 and HD3 on the bias voltage is demonstrated in Fig. 5.20. HD3 $<-70 \mathrm{~dB}$ and HD2 $<-90 \mathrm{~dB}$ are 


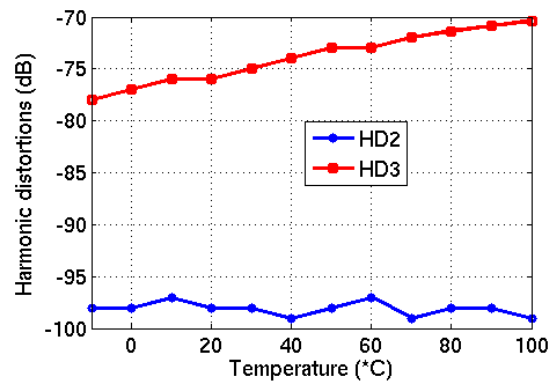

Figure 5.21: HD3and HD2 versus temperature.

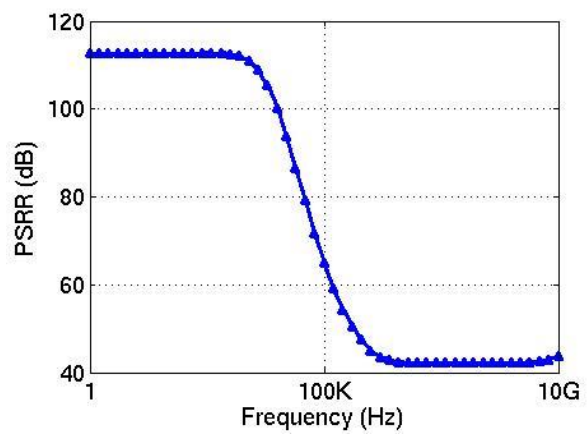

Figure 5.22: PSRR with 5\% mismatch.

achieved with the change of bias voltage from $0.95 \mathrm{~V}$ to $1.25 \mathrm{~V}$. The temperature variation is also verified and shown in Fig. 5.21. HD2 is not sensitive to temperature whereas HD3 is always less than $-70 \mathrm{~dB}$ in the range of $-10-100{ }^{\circ} \mathrm{C}$.

The power supply rejection ratio (PSRR) is verified for a sinusoidal disturbance imposed on $V_{d d}$ over a wide frequency range as shown in Fig. 5.22. With intentionally introduced $5 \%$ mismatch into two branches of the differential circuit, PSRR is always greater than $40 \mathrm{~dB}$.

The SC array is designed based on the available design area $\mathrm{W}-\mathrm{C}_{\mathrm{u}}$ for given resolution and signal bandwidth. For 7- and 8-bit applications with $3 \mathrm{GHz}$ clock, according to Fig. 5.9 and Fig. 5.10 we choose $C_{u}=200 \mathrm{fF}$ and the total switch size $W=$ $64 \mu \mathrm{m}(16 \mu \mathrm{m}$ for NMOS and $48 \mu \mathrm{m}$ for PMOS) with $L=60 \mathrm{~nm}$. The necessary dummies of a similar size are applied.

The simulation results of the complete SC DAC for 7- and 8-bit resolution are shown in Fig. 5.23 for OSR $=1$.1. For frequencies above $3.5 \mathrm{GHz}$ the SFDR drops due to violation of the settling time. The attained SFDR is not affected by the output driver for its high linearity and bandwidth. 


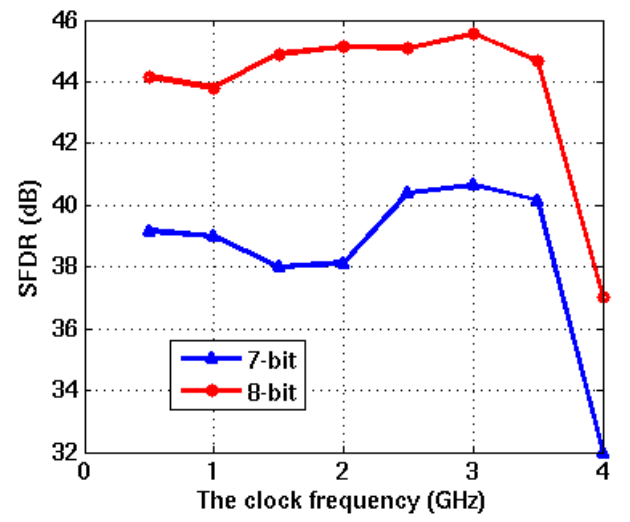

Figure 5.23: SFDR against clock frequency for 7-bit and 8-bit $(\mathrm{OSR}=1.1)$.

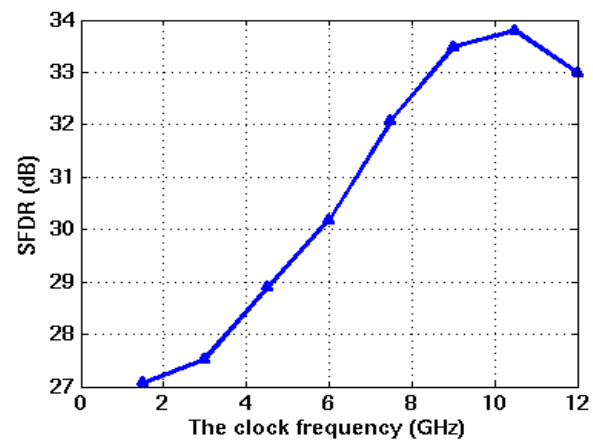

Figure 5.24: SFDR against clock frequency $(\mathrm{OSR}=1.1)$ for $n=6$.

For 6-bit resolution with $12 \mathrm{GHz}$ clock frequency the unit capacitance value must be largely reduced according to the available design area shown in Fig. 5.11. In this case we choose $C_{u}=30 \mathrm{fF}$ while using the same size of switches $(W=64 \mu \mathrm{m})$. The simulation results of the complete SC DAC with 6-bit resolution are shown in Fig. 5.24 for OSR $=1.1$. The attained SFDR approaches $34 \mathrm{~dB}$ but for clock frequencies below $8 \mathrm{GHz}$ it is largely deteriorated due to leakage effects caused by too low time constant $R_{o f f} C_{u}$. In fact, this could be seen as the lower and upper bounds on $C_{u}$, shown in Fig. 5.11 , to go up for lower clock frequencies while the actual operating point $\left(C_{u}, W\right)$ remains unchanged and ultimately it falls out of the design area.

The DAC power consumption is mostly due to the output driver and the digital clock part $\left(75 \mathrm{~mW} @ f_{s}=3 \mathrm{GHz}\right)$. The pipeline SC array consumes much less power $(15 \mathrm{~mW}$ @ $f_{s}=3 \mathrm{GHz}$ ) since its total capacitance is relatively low and the operation is based on 
Table 5.1: Summary of SC DAC performance

\begin{tabular}{l|l|l|l} 
Technology & \multicolumn{2}{|l}{$65 \mathrm{~nm}$ CMOS } \\
\hline Supply voltage & \multicolumn{2}{|l}{$1.2 \mathrm{~V}$} \\
\hline Power consumption & \multicolumn{2}{|l}{$90 \mathrm{~mW} @ f_{s}=3 \mathrm{GHz}$} \\
\hline Resolution & 8 -bit & 7 -bit & 6-bit \\
\hline SFDR $[\mathrm{dB}]$ & 45 & 40 & 33 \\
\hline$f_{s}[\mathrm{GHz}]$ & 3 & 3 & 12 \\
\hline$f_{\text {in }}[\mathrm{GHz}]$ & 1.36 & 1.36 & 5.5
\end{tabular}

charge redistribution. The summary of the SC DAC performance is presented in Table 5.1 .

There are 5 definitions of figure of merit (FOM) in [53, 103] but only two of them are generic i.e. suitable for any kind of DAC type. They are FOM2 and FOM3

$$
\begin{aligned}
& \text { FOM } 2=\frac{2^{N} B W_{N}}{P_{\text {total }}} \\
& \text { FOM } 3=\frac{V_{\text {swing }}}{P_{\text {total }}} B W 10^{\frac{\text { SFDR }}{20}}
\end{aligned}
$$

where $N$ is the number of bits, $B W$ (or $B W_{N}$ ) is the signal bandwidth, $P_{\text {total }}$ is the total power consumption, whereas $V_{\text {swing }}$ and $S F D R$ stand for the differential output swing and spurious free dynamic range of DACs, respectively.

Table 5.2 gives performance comparison of the presented design and other state-ofthe-art publications. 
Table 5.2: Performance comparison of SC DACs

\begin{tabular}{|c|c|c|c|c|c|c|c|c|c|}
\hline Ref & $\begin{array}{c}\text { This } \\
\text { work }\end{array}$ & $\begin{array}{c}{[62]} \\
\text { ASSC } \\
2010\end{array}$ & $\begin{array}{c}{[61]} \\
\text { JSSC2004 }\end{array}$ & $\begin{array}{c}\text { [106] } \\
\text { TCAS } \\
2013\end{array}$ & $\begin{array}{c}{[104]} \\
\text { ISSC } \\
2011\end{array}$ & $\begin{array}{r}{[54]} \\
\text { JSSC } \\
2009\end{array}$ & $\begin{array}{c}{[105]} \\
\text { JSSC } \\
2008\end{array}$ & $\begin{array}{c}{[53]} \\
\text { JSSC } \\
2008\end{array}$ & $\begin{array}{c}{[86]} \\
\text { JSSC } \\
2001\end{array}$ \\
\hline $\begin{array}{c}\text { DAC } \\
\text { type }\end{array}$ & $\mathrm{SC}$ & $\mathrm{SC}$ & $\mathrm{SC}$ & $\Delta \Sigma$ & $\begin{array}{l}\text { Current } \\
\text { Source }\end{array}$ & $\begin{array}{l}\text { Current } \\
\text { Source }\end{array}$ & $\begin{array}{l}\text { Current } \\
\text { Source }\end{array}$ & $\begin{array}{l}\text { Current } \\
\text { Source }\end{array}$ & $\begin{array}{c}\text { Current } \\
\text { Source }\end{array}$ \\
\hline $\begin{array}{l}\text { Tech. } \\
\text { [nm] }\end{array}$ & 65 & 90 & 500 & 65 & 65 & 65 & 90 & 130 & 350 \\
\hline$\underset{\text { SV] }}{\text { Supply }}$ & 1.2 & $2.5 / 1.2$ & 3.3 & $1.2 / 1$ & $2.5 / 1.1$ & 2.5 & $1.8 / 1$ & 1.2 & $3 / 1.9$ \\
\hline $\begin{array}{c}\text { Fclk } \\
{[\mathrm{GHz}]}\end{array}$ & $3 / 12$ & 0.8 & 0.2 & 8 & 56 & 2.9 & 12 & 3 & 1 \\
\hline $\begin{array}{c}\text { BW } \\
{[\mathbf{G H z}]}\end{array}$ & $1.38 / 5.5$ & 0.4 & $34 \mathrm{MHz}$ & 0.2 & 26.9 & 0.55 & 1.5 & 1.426 & 0.49 \\
\hline $\begin{array}{c}\text { SFDR } \\
\text { [dB] }\end{array}$ & $45 / 33$ & 53 & 70 & $\begin{array}{c}26 \\
\text { SNDR }\end{array}$ & 30 & 60 & 35 & 36.2 & 61.2 \\
\hline $\begin{array}{l}\text { IM3 } \\
{[\mathrm{dB}]}\end{array}$ & $\begin{array}{l}-45 / \\
-33\end{array}$ & - & - & -57 & - & -60 & - & -39.5 & - \\
\hline $\begin{array}{c}\text { Swing } \\
\text { [Vppd] }\end{array}$ & $0.3 / 0.3$ & 2.9 & 2 & 0.3 & 0.6 & 2.5 & 1.6 & 0.4 & 1.6 \\
\hline $\begin{array}{c}\text { Power } \\
{[\mathrm{mW}]}\end{array}$ & $90 / 130$ & 103 & 693 & 68 & 750 & 188 & 190 & 29 & 110 \\
\hline Bits & $8 / 6$ & 12 & 10 & 12 & 6 & 12 & 8 & 6 & 10 \\
\hline $\begin{array}{c}\text { FOM2 } \\
{\left[10^{12}\right]}\end{array}$ & $3.9 / 2.7$ & 15.9 & 0.05 & 12 & 2.3 & 12 & 2.02 & 3.2 & 4.6 \\
\hline $\begin{array}{c}\text { FOM3 } \\
{\left[10^{12}\right]}\end{array}$ & $1.4 / 1$ & 5 & 0.3 & - & 0.7 & 7.3 & 0.71 & 1.3 & 8.2 \\
\hline
\end{tabular}

${ }^{\text {a) Simulation. }}$ 


\section{Chapter 6}

\section{A 1-GHz Bandwidth 12-bit SC DAC for 60-GHz Radio in 65-nm CMOS}

\subsection{Introduction}

As mentioned in Chapter 5, this Chapter is the second work in Part II. It is about design and implementation of 1-GHz bandwidth 12-bit SC DAC for $60-\mathrm{GHz}$ radio in 65 nm CMOS technology.

The typical capacitive DAC architectures used in [61-63] consist of the main SC array and output driver/buffer to drive the off-chip load (50 $\Omega$ ). However, if implemented in a fully integrated transceiver [47] DAC does not need output driver any more since SC arrays can drive directly filters/mixers as on-chip loads. 
The main part of capacitive DACs is SC array. In the latest works of high speed capacitive DACs, the pipeline architecture of SC array was used [61, 62]. The timeinterleaved topology of pipeline SC was utilized to improve speed of DAC [62]. However, it can only work up to $800 \mathrm{MS} / \mathrm{s}$ at Nyquist rate and the finite bandwidth of the track-and-hold circuit causes residual dynamic nonlinearities since the kT/C noise prevents the hold capacitor from being chosen small value which is needed for high speed operation. Moreover, based on the earlier study we have found that the pipeline capacitor array suffers from clock feed-through effect for high resolution (12-bit) DACs [63]. It also needs multiple-phase clock which is generated on-chip and resulting in some amount of power dissipation for DACs. In order to fix the clock feed-through problem we propose a split-segmented SC array topology since it can get rid of pipelined charge propagating. Moreover, without using multiple-phase clock for the proposed SC array can save some power and avoid sensitivity problem of multiple phases. The split-segmented SC array can work at even higher speed (5 GS/s) since it is not limited by kT/C noise due to absence of the track-and-hold circuit.

Another important part of capacitive DACs is output driver. The ability to drive offchip load of SC arrays is poor, so a suitable output driver is necessary. This driver appears critical for linearity of a capacitive DAC when the high output voltage swing is required [62]. One solution to this problem is using a closed-loop architecture where the circuit linearity is improved by negative feedback [61]. However, the performance of this technique cannot maintain at high frequencies $(>1 \mathrm{GHz})$ due to the loop bandwidth limitation. Another solution is an open-loop driver design that can be supported by a pre-distortion calibration [62]. However, the latter is usually limited by an assumption of minimum memory effect of the nonlinear object and high accuracy of analog-todigital converter (ADC). In order to design a driver to be able to work at high frequency (> $4 \mathrm{GHz}$ ) without using any form of calibration, we propose a linear open-loop driver which is supported with circuit technique of derivative superposition (DS) [107] for canceling both second and third-order harmonic distortion to drive the proposed splitsegmented SC array.

For the bandwidth greater than $1 \mathrm{GHz}$, no implementation of SC DAC has been recently reported. This work is one of the first design and implementation of SC DACs which achieves the highest reported bandwidth, to the authors' knowledge. This paper presents the design, analysis and implementation of a 12-bit split-segmented SC DAC in $65 \mathrm{~nm}$ CMOS technology. Design and analysis of the output driver using Volterra Series [101] are also presented. Measurement results of the complete 12-bit SC DAC at $5 \mathrm{GS} / \mathrm{s}$ achieved spurious free dynamic range (SFDR) of $44 \mathrm{~dB}$ for $1 \mathrm{GHz}$ signal bandwidth while consuming $50 \mathrm{~mW}$ from $1 \mathrm{~V}$ digital/1.2 $\mathrm{V}$ analog supplies. The proposed design can be used for 60-GHz radio baseband since it satisfies the spectral mask of the IEEE 802.11ad WiGig standard with a second-order reconstruction filter. Moreover, similar performance has achieved with $0.9 \mathrm{~V}$ supply, therefore it shows scalability for more advanced technology. 


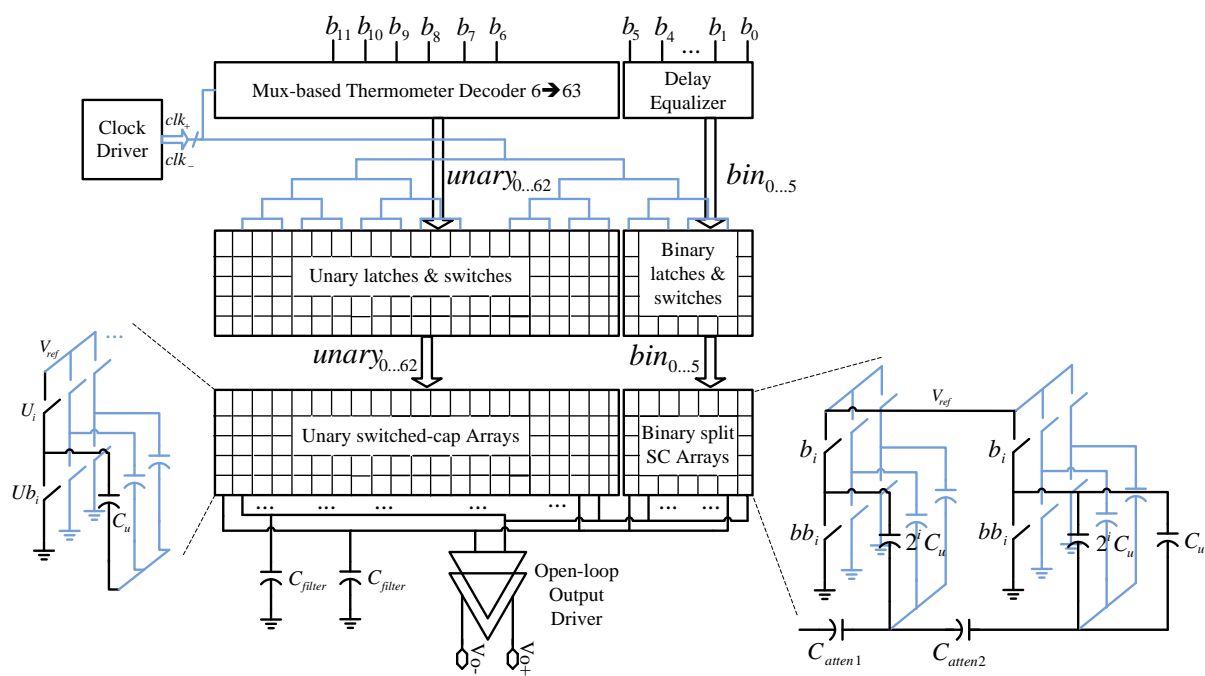

Figure 6.1: The 12-bit split-segmented SC DAC architecture.

\subsection{A 12-bit Split-segmented SC DAC Architecture}

The proposed capacitive DAC architecture is shown in Fig. 6.1. The SC DAC uses the 6-3-3 split-segmented SC arrays which have 6 most significant bits (MSBs) of thermometer codes, 6 least significant bits (LSBs) of binary-weighted ones with 2 attenuated capacitors in the middle for reducing capacitance spread [60]. The mux-based 6-63 thermometer decoder is used to convert $6 \mathrm{MSBs}$ of the input to 63 unary bits since it is more suitable for high speed than the logic-based one [52]. The SC cell is based on capacitor/voltage divider and linearly proportional with supply $\mathrm{V}_{\text {ref }}$, unit capacitance $C_{u}$, output capacitance $C_{\text {filter }}(6.1-6.2)$. For given specification of 12 bits, $\mathrm{f}_{\mathrm{s}}>3 \mathrm{GHz}, \mathrm{BW}>1$ $\mathrm{GHz}$, the size of switches $W / L$ is chosen in the way that it satisfies the upper bound of SSN (in section 6.5) and the lower bound of settling time (in section 6.4). Similarly the unit capacitance $C_{u}$ is designed based on the requirement of both upper bound from settling time, capacitance parasitic effect (6.37) and lower bound from kT/C noise and capacitor mismatch in section 6.3. The internal node between switch $U_{i}$ and $U b_{i}$ in Fig. 6.1 does not have clock feed-through voltage existing since when one of these switches is off, another will be on to create the charged/discharged path to $\mathrm{V}_{\text {ref }} / \mathrm{GND}$. The linear output driver which will be described in details in Section VI is used to drive off-chip load (50 $\Omega$ matching from equipment). 
Table 6.1: The truth table for 3-8 decoder: Mux (a), 2-4 Select generator (b).

\begin{tabular}{|c|c|c|c|c|}
\multicolumn{7}{|c|}{ (a) } \\
\hline$S$ & $I$ & $Y$ & $X$ & $U$ \\
\hline$S_{0}$ & $b_{6}$ & 0 & $S_{0}$ & $d_{0}$ \\
\hline$S_{0}$ & 0 & 0 & $S_{0}$ & $d_{1}$ \\
\hline$S_{1}$ & $b_{6}$ & $S_{0}$ & $\overline{b_{8}}$ & $d_{2}$ \\
\hline$S_{1}$ & 0 & $S_{0}$ & $\overline{b_{8}}$ & $d_{3}$ \\
\hline$S_{2}$ & $b_{6}$ & $\overline{b_{8}}$ & $\overline{S_{3}}$ & $d_{4}$ \\
\hline$S_{2}$ & 0 & $\overline{b_{8}}$ & $\overline{S_{3}}$ & $d_{5}$ \\
\hline$S_{3}$ & $b_{6}$ & $\overline{S_{3}}$ & 1 & $d_{6}$ \\
\hline$S_{3}$ & 0 & $\overline{S_{3}}$ & 1 & $d_{7}$ \\
\hline
\end{tabular}

(b)

\begin{tabular}{|c|c|c|c|c|}
\hline \multicolumn{4}{|c|}{$b_{[7: 8]}$} & \multirow{2}{*}{$S$} \\
\hline 11 & 10 & 01 & 00 & \\
\hline 0 & 0 & 0 & 1 & $S_{0}$ \\
\hline 0 & 0 & 1 & 0 & $S_{1}$ \\
\hline 0 & 1 & 0 & 0 & $S_{2}$ \\
\hline 1 & 0 & 0 & 0 & $S_{3}$ \\
\hline
\end{tabular}

Neglecting all the parasitic capacitances for simplicity the output voltage of SC arrays is linearly dependent on the values of input data $U_{i}, b_{i}$, supply $\mathrm{V}_{\text {ref }}$, unit capacitance $C_{u}$, output capacitance $C_{\text {filter }}$ as

$$
V_{\text {out }}=V_{\text {ref }} k\left(\sum_{i=0}^{62} U_{i}+\frac{1}{2^{3}-1}\left(\sum_{i=0}^{2} b_{i} 2^{i}+\frac{1}{2^{3}-1} \sum_{i=0}^{2} b_{i} 2^{i}\right)\right)
$$

where

$$
k=\frac{C_{u}}{\left(64 C_{u}+C_{\text {filter }}\right)}
$$

\section{Design of mux-based 6-63 thermometer decoder:}

The mux-based 6-63 decoder is designed based on the work which was proposed in [52] to encode 6 binary-weighted MSBs $b_{[6: 11]}$ to 63 unary bits. In this design we minimize the use of conventional logic gates and maximize using MUX due to its fast operation as shown in Fig. 6.2. The 3 MSBs $b_{[9: 11]}$ are converted to select-signals $S_{[0: 7]}$ by the 3-8 select generator while others $b_{[6: 8]}$ go to the 3-8 decoder. Similarly the 3-8 decoder is designed based on MUX with the combination of the 2-4 select generator and $b_{6}$ to generate data $d_{[0: 7]}$ and using the truth tables shown in Table 6.1. The MUX is developed from the one in [55] with using transmission gates for the switch of selectsignal $S$. The inverted select-signal controls PMOS of switch while the NMOS is controlled by non-inverted signal with the delay equalizing circuit. The final 63 unary bits $U_{[0: 62]}$ are generated from those data $d_{[0: 7]}$ and select-signal $S_{[0: 7]}$ based on the truth tables in Table 6.2. 


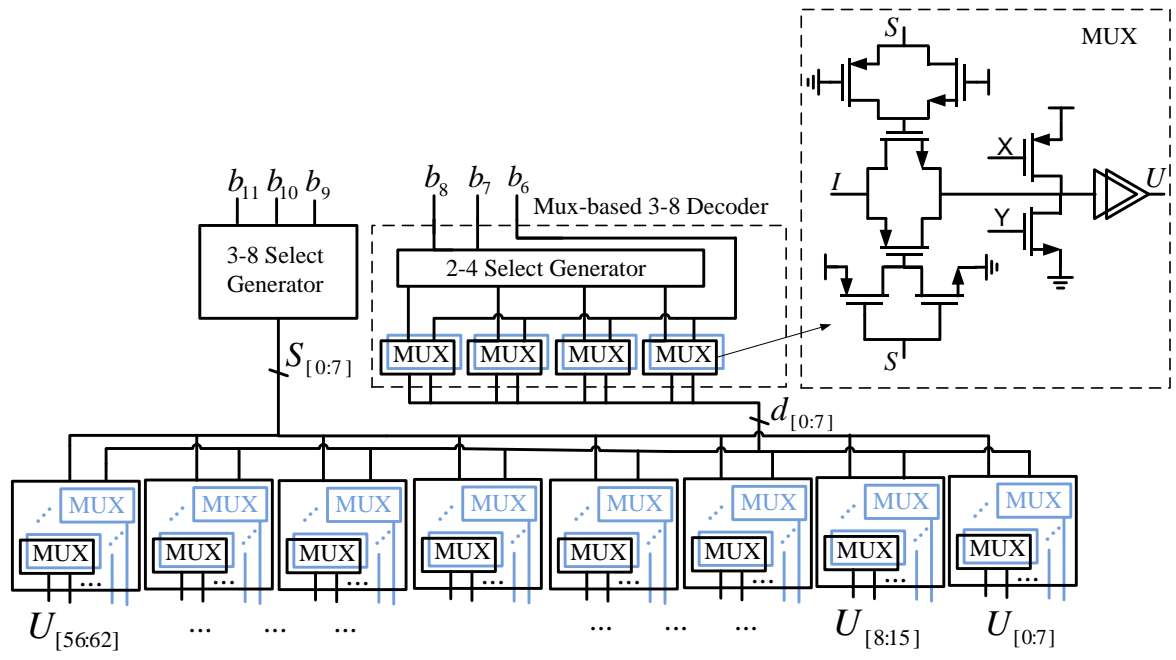

Figure 6.2: The Mux-based 6-63 decoder.

Table 6.2: The truth table for 6-63 decoder: Mux (a), 3-8 Select generator (b)

(a)

\begin{tabular}{|c|c|c|c|c|}
\hline$S$ & $I$ & $Y$ & $X$ & $U$ \\
\hline$S_{0}$ & $d_{[0: 7]}$ & 0 & $S_{0}$ & $U_{[0: 7]}$ \\
\hline$S_{1}$ & $d_{[0: 7]}$ & $S_{0}$ & $\overline{b_{10}+b_{11}}$ & $U_{[8: 15]}$ \\
\hline$S_{2}$ & $d_{[0: 7]}$ & $\overline{b_{10}+b_{11}}$ & $S_{0}+S_{1}+S_{2}$ & $U_{[16: 23]}$ \\
\hline$S_{3}$ & $d_{[0: 7]}$ & $S_{0}+S_{1}+S_{2}$ & $\overline{b_{11}}$ & $U_{[24: 31]}$ \\
\hline$S_{4}$ & $d_{[0: 7]}$ & $\overline{b_{11}}$ & $\overline{b_{11}}+S_{4}$ & $U_{[32: 39]}$ \\
\hline$S_{5}$ & $d_{[0: 7]}$ & $\overline{b_{11}}+S_{4}$ & $\overline{S_{6}+S_{7}}$ & $U_{[00: 47]}$ \\
\hline$S_{6}$ & $d_{[0: 7]}$ & $\overline{S_{6}+S_{7}}$ & $\overline{S_{7}}$ & $U_{[48: 55]}$ \\
\hline$S_{7}$ & $d_{[0: 7]}$ & $\overline{S_{7}}$ & 1 & $U_{[56: 62]}$ \\
\hline
\end{tabular}

(b)

\begin{tabular}{|c|c|c|c|c|c|c|c|c|}
\hline \multicolumn{7}{|c|}{$b_{[9: 11]}$} & \multirow{2}{*}{$S$} \\
\hline 111 & 110 & 101 & 100 & 011 & 010 & 001 & 000 & \\
\hline 0 & 0 & 0 & 0 & 0 & 0 & 0 & 1 & $S_{0}$ \\
\hline 0 & 0 & 0 & 0 & 0 & 0 & 1 & 0 & $S_{1}$ \\
\hline 0 & 0 & 0 & 0 & 0 & 1 & 0 & 0 & $S_{2}$ \\
\hline 0 & 0 & 0 & 0 & 1 & 0 & 0 & 0 & $S_{3}$ \\
\hline 0 & 0 & 0 & 1 & 0 & 0 & 0 & 0 & $S_{4}$ \\
\hline 0 & 0 & 1 & 0 & 0 & 0 & 0 & 0 & $S_{5}$ \\
\hline 0 & 1 & 0 & 0 & 0 & 0 & 0 & 0 & $S_{6}$ \\
\hline 1 & 0 & 0 & 0 & 0 & 0 & 0 & 0 & $S_{7}$ \\
\hline
\end{tabular}




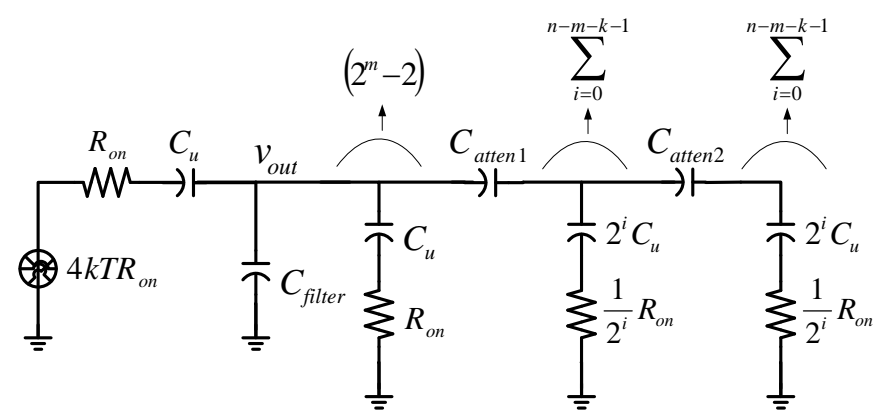

Figure 6.3: Model of thermometer switch noise.

\subsection{The Analysis of kT/C Noise and Capacitor Mismatch in SC Array}

In order to choose the right values for the switch sizes and unit capacitance this section describes the analysis of $\mathrm{kT} / \mathrm{C}$ noise and capacitor mismatch in the 12-bit splitsegmented SC arrays. Especially it proves the feasibility of 12-bit SC DAC to work at 3 $\mathrm{GS} / \mathrm{s}$ and not limited by those factors. Moreover the lower bounds of $C_{u}$ due to $\mathrm{kT} / \mathrm{C}$ noise and capacitor mismatch can be found through this analysis.

\subsubsection{Thermal noise analysis}

Noise contribution from all switches to the output can be estimated using the superposition principle.

Noise due to thermometer SC arrays:

From A.6.1-A.6.7 in the appendix F, the total admittance of thermometer, binary SC arrays and load $\left(C_{\text {filter }}\right)$ in Fig. 6.3 is found as

$$
Y_{1 \sum}=\frac{1}{Z_{\text {b1atten } 1}}+Y_{t h 1}+j \omega C_{f i l t e r} .
$$

The power spectral density (PSD) noise at output due to each thermometer branch is

$$
S_{t h}(f)=4 k T R_{o n} \times\left|\frac{\frac{1}{Y_{1 \Sigma}}}{\frac{1}{Y_{1 \Sigma}}+\frac{1}{j \omega C_{u}}+R_{o n}}\right|^{2}
$$




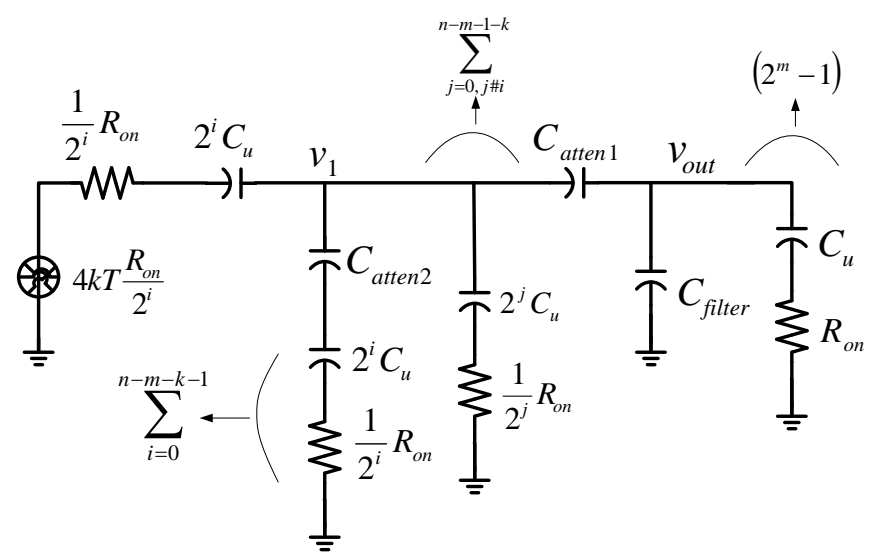

Figure 6.4: Model of switch noise for the first binary segment.

The total power spectral density (PSD) noise at output due to all thermometer branches is calculated as

$$
S_{t h \sum}(f)=\left(2^{m}-1\right) S_{t h}(f)
$$

\section{Noise due to the first binary SC arrays:}

From A.6.8-A.6.15 in the appendix F, the voltage gain from noise source to the output in Fig. 6.4 can be calculated as

$$
\begin{gathered}
A_{V 1}=\frac{v_{\text {out }}}{v_{n s}}=\frac{v_{\text {out }}}{v_{1}} \frac{v_{1}}{v_{n s}} \\
\frac{v_{\text {out }}}{v_{1}}=\frac{\frac{1}{Y_{2 \text { load }}}}{\frac{1}{Y_{2 \text { load }}}+\frac{1}{j \omega C_{\text {atten } 1}}} \\
\frac{Z_{\text {b2atten } 2}\left\|Z_{\text {b2a }}\right\| Z_{\text {load_atten }}}{v_{n s}}=\frac{R_{\text {on }}}{Z_{\text {b2atten } 2}\left\|Z_{b 2 a}\right\| Z_{\text {load_atten }}+\frac{1}{2^{i}}}
\end{gathered}
$$




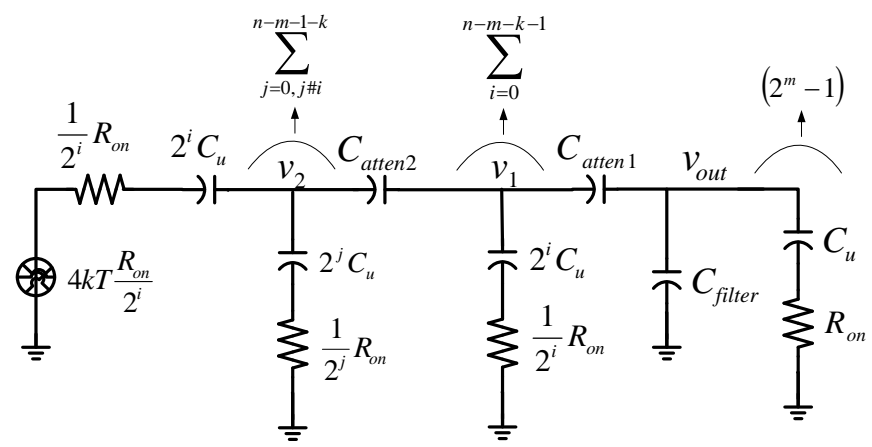

Figure 6.5: Model of switch noise for the second binary segment.

with

$$
Z_{\text {load_atten }}=\frac{1}{Y_{2 l o a d}}+\frac{1}{j \omega C_{\text {atten } 1}}
$$

The power spectral density (PSD) noise at output due to each branch in the first binary segment is found

$$
S_{b i n 1}(f)=\frac{4 k T R_{o n}}{2^{i}}\left|A_{V 1}\right|^{2}
$$

The total power spectral density (PSD) noise at output due to the first binary segment is as

$$
S_{b i n 1} \sum(f)=\sum_{i=0}^{n-m-k-1} S_{b i n 1}(f)
$$

\section{Noise due to the second binary SC arrays:}

Similarly the voltage gain from noise source to output in Fig. 6.5 is calculated based on A.6.16-A.6.23

$$
\begin{gathered}
A_{V 2}=\frac{v_{\text {out }}}{v_{n s}}=\frac{v_{\text {out }}}{v_{1}} \frac{v_{1}}{v_{2}} \frac{v_{2}}{v_{n s}} \\
\frac{v_{\text {out }}}{v_{1}}=\frac{\frac{1}{Y_{\text {3load }}}}{\frac{1}{Y_{\text {3load }}}+\frac{1}{j \omega C_{\text {atten } 1}}}
\end{gathered}
$$




$$
\frac{v_{1}}{v_{2}}=\frac{Z_{b 31} \| Z_{\text {load_atten }}}{Z_{b 31} \| Z_{\text {load_atten }}+\frac{1}{j \omega C_{\text {atten } 2}}}
$$

with

$$
\begin{aligned}
& Z_{\text {load_atten }}=\frac{1}{Y_{3 \text { load }}}+\frac{1}{j \omega C_{\text {atten } 1}} \\
& \frac{v_{1}}{v_{n s}}=\frac{Z_{b 32} \| Z_{\text {load_atten } 2}}{Z_{b 32} \| Z_{\text {load_atten } 2}+\frac{R_{o n}}{2^{i}}+\frac{1}{j \omega C_{u} 2^{i}}}
\end{aligned}
$$

where

$$
Z_{\text {load_atten } 2}=Z_{\text {b31a }} \| Z_{\text {load_atten }}+\frac{1}{j \omega C_{\text {atten } 2}}
$$

The power spectral density (PSD) noise at output due to each branch in the second binary segment is found

$$
S_{b i n 2}(f)=\frac{4 k T R_{o n}}{2^{i}}\left|A_{V 2}\right|^{2}
$$

The total power spectral density (PSD) noise at output due to the second binary segment is as

$$
S_{b i n 2 \sum}(f)=\sum_{i=0}^{n-m-k-1} S_{b i n 2}(f)
$$

\section{Noise due to all binary and thermometer SC arrays:}

The total power spectral density (PSD) noise at output is sum of all individual noise sources as

$$
S_{k T / C \sum}(f)=S_{t h \sum}(f)+S_{b i n 1 \sum}(f)+S_{b i n 2 \sum}(f)
$$

Fig. 6.6 shows a comparison of SC noise PSD obtained with (6.20) and by a circuit simulation model using SpectreRFTM. The results are achieved by Pnoise simulation followed by spectrum averaging at low frequencies. Using transient noise analysis the same result is obtained. The PSD depends on values of on-resistance $R_{\text {on }}$ and is almost independent on frequency because the noise of switch is transferred to the output when it conducts in half of cycle. 


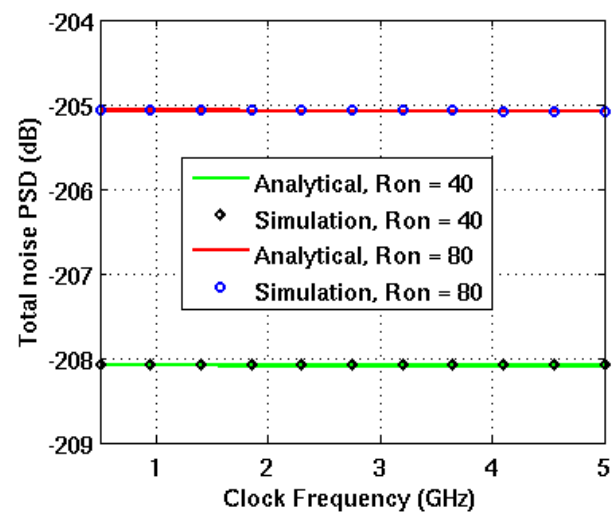

Figure 6.6: SC noise PSD comparison of analytical model (6.20) and SpectreRFTM simulations with $C_{u}=30 \mathrm{fF}, R_{\text {on }}=40 / 80 \Omega, C_{\text {filter }}=3 \mathrm{pF}$.

Generally, the kT/C noise should be less than quantization noise of DAC with certain resolution. For n-bit DAC, the quantization noise PSD can be calculated from

$$
S_{Q}(f)=\frac{V_{F S}^{2}}{12\left(2^{n}-1\right)^{2} B W}
$$

where $V_{F S}$ is the full-scale voltage, $n$ is the number of bits, and $B W=f_{s} / 2$ for Nyquist rate, the minimum capacitance value $C_{u}$ can be estimated using (6.20)

$$
S_{k T / C \sum}(f)<\frac{V_{F S}^{2}}{12 B W\left(2^{n}-1\right)^{2}}
$$

For $\mathrm{f}_{\mathrm{s}}=3 \mathrm{GHz}, \mathrm{V}_{\mathrm{FS}}=0.8 \mathrm{~V}, \mathrm{C}_{\text {filter }}=3 \mathrm{pF}$, Ron $<80 \Omega$ the PSD of $\mathrm{kT} / \mathrm{C}$ noise is more than $20 \mathrm{~dB}$ less than the one of 12-bit quantization noise as shown in Fig. 6.7. Using the upper bound of $R_{\text {on }}=1.85 \mathrm{~K} \Omega$ due to settling time (6.32) with $\mathrm{n}=12, f_{s}=3 \mathrm{GHz}$, the condition in (6.22) is still satisfied with more than $13 \mathrm{~dB}$ margin. Therefore $\mathrm{kT} / \mathrm{C}$ noise is not one of critical considerations of designing 12-bit SC DAC. 


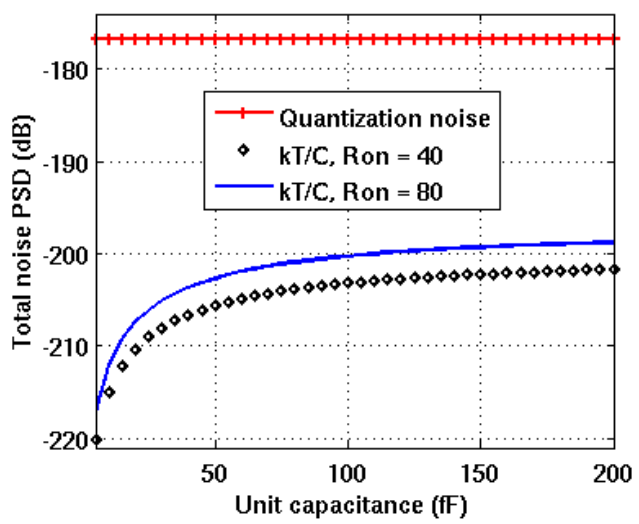

Figure 6.7: SC PSD comparison of kT/C noise (6.20) with $R_{o n}=40 \Omega, 80 \Omega$ and quantization noise $(6.21), \mathrm{f}_{\mathrm{s}}=3 \mathrm{GHz}, \mathrm{V}_{\mathrm{FS}}=0.8 \mathrm{~V}, \mathrm{C}_{\text {filter }}=3 \mathrm{pF}, \mathrm{n}=12$.

\subsubsection{Capacitor mismatch analysis}

Following the analysis in [99] an error voltage due to capacitor mismatch can be attained

$$
V_{D N L}=\frac{C_{u}+\left(\sqrt{2^{n-1}}-\sqrt{2^{n-1}-1}\right) \Delta C_{u}}{2^{n} C_{u}+\sqrt{2^{n}} \Delta C_{u}} V_{F S}-\frac{V_{F S}}{2^{n}}
$$

where $V_{F S}$ is the full-scale output range and $V_{D N L}$ is the differential nonlinearity of DAC. The standard deviation $\sigma\left(\Delta C_{u} / C_{u}\right)$ of capacitor mismatch for a typical metal capacitor can be found from [109]

$$
\sigma\left(\frac{\Delta C_{u}}{C_{u}}\right)=\frac{K_{\sigma}}{\sqrt{A}} \quad \text { and } \quad C_{u}=K_{C} A,
$$

where $A$ is the capacitor area, while $K_{\sigma}$ (matching coefficient) and $K_{C}$ (capacitance density) are technology constants provided by the chip manufacturer.

The standard variation of $V_{D N L}$ would be

$$
\sigma\left(V_{D N L}\right)=\frac{1+\left(\sqrt{2^{n-1}}-\sqrt{2^{n-1}-1}\right) \sigma\left(\frac{\Delta C_{u}}{C_{u}}\right)}{2^{n}+\sqrt{2^{n}} \sigma\left(\frac{\Delta C_{u}}{C_{u}}\right)} V_{F S}-\frac{V_{F S}}{2^{n}}
$$

From (6.24), we have 


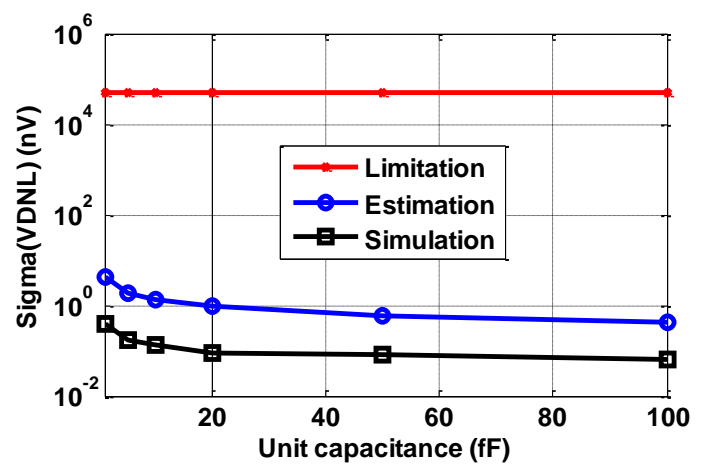

Figure 6.8: $\mathrm{V}_{\mathrm{DNL}}$ standard deviation comparison of estimated model (6.27) and SpectreRF ${ }^{\mathrm{TM}} \mathrm{MC}$ simulations against 1/6 LSB voltage of 12 bit DAC.

$$
\sigma\left(\frac{\Delta C_{u}}{C_{u}}\right)=\frac{K_{\sigma} \sqrt{K_{C}}}{\sqrt{C_{u}}}
$$

Substituting (6.26) to (6.25), we have

$$
\sigma\left(V_{D N L}\right)=\frac{1+\left(\sqrt{2^{n-1}}-\sqrt{2^{n-1}-1}\right) \frac{K_{\sigma} \sqrt{K_{C}}}{\sqrt{C_{u}}}}{2^{n}+\sqrt{2^{n}} \frac{K_{\sigma} \sqrt{K_{C}}}{\sqrt{C_{u}}}} V_{F S}-\frac{V_{F S}}{2^{n}}
$$

With $65 \mathrm{~nm}$ CMOS process of STM foundry we have $K_{\sigma}=0.5 \% \mu \mathrm{m}, K_{C}=0.39$ $\mathrm{fF} / \mu_{\mathrm{m}}^{2}$ for metal-to-metal capacitor. Fig. 6.8 gives a comparison of $\mathrm{V}_{\mathrm{DNL}}$ standard deviation obtained with model (6.27) and SpectreRF ${ }^{\mathrm{TM}}$ simulations. The simulation results are achieved by statistical Monte-Carlo analysis only with the focus on capacitor variation whereas the switches are assumed ideal and the simulation count is 100. A very low clock frequency was used to verify the static $\mathrm{V}_{\mathrm{DNL}}$ with input data of MSB transition [99]. We usually use $3 \sigma$ standard, meaning that $3 \sigma\left(V_{D N L}\right)$ should be less than a half of $\mathrm{V}_{\mathrm{LSB}}$. The limitation line shown in Fig. 6.8 is therefore one sixth of $\mathrm{V}_{\mathrm{LSB}}$ of 12bit resolution DAC which is $32 \mu \mathrm{V}$ with $V_{F S}=0.8 \mathrm{~V}$. The $\mathrm{V}_{\mathrm{DNL}}$ standard deviations are in nano-volt range while those from simulation are by one order of magnitude less than this. The results from simulation are lower than the ones from estimation because in calculation we assumed total variation of mismatch as maximum [99] while the variation of unit capacitors mismatch in Monte-Carlo simulation is randomly distributed. Importantly, the both values are by orders of magnitude less than the limitation voltage of 12-bit DAC. Therefore the limit due to the capacitor mismatch can be neglected for 12-bit resolution and metal-to-metal unit capacitor. 
In order to calculate the bound of unit capacitance, the error voltage in (6.23) can be simplified

$$
V_{D N L} \approx \frac{\sqrt{2^{n}-1}}{2^{n}} \frac{\Delta C_{u}}{C_{u}} V_{F S}
$$

From (26) and (28), the standard deviation of the voltage error caused by capacitor mismatch is found as

$$
\sigma\left(V_{D N L}\right) \approx \frac{\sqrt{2^{n}-1}}{2^{n}} V_{F S} \sigma\left(\frac{\Delta C_{u}}{C_{u}}\right)
$$

For the DAC accuracy, it is common to maintain $3 \sigma\left(V_{D N L}\right)$ less than a half of LSB. Hence, the lower bound for the unit capacitor due to the mismatch in $n$-bit DAC is

$$
C_{u}>9 K_{C} K_{\sigma}^{2}\left(2^{n}-1\right)
$$

For $65 \mathrm{~nm}$ CMOS process with metal-to-metal capacitor, we have $K_{\sigma}=0.5 \% \mu \mathrm{m}, K_{C}$ $=0.39 \mathrm{fF} / \mu \mathrm{m}^{2}$ that for 12-bit DAC give the unit capacitor $C_{u}>0.4 \mathrm{fF}$.

\subsection{Settling Time Analysis and Parasitic Effects}

The settling time is one of the critical limits and needs to be analyzed for the high speed operation of SC array. The upper bound of unit capacitance and the lower bound of switch sizes are estimated through this settling time analysis. Furthermore the capacitance parasitic has an effect on it and even narrows down more the design range of $C_{u}$. Their details can be seen in the following description.

\subsubsection{Settling time analysis}

Due to imperfections of the transistor switches $\left(R_{o f f} / R_{o n}\right.$ resistances) the leakage and incomplete settling in the capacitor array result in voltage errors which degrade DAC linearity [96]. Firstly the upper bound of on-resistance of switch is estimated. When switch is on, the time-constant will be $R_{o n} C_{u}$ due to $C_{\text {filter }} \gg C_{u}$. The maximum charged/discharged voltage error due to settling time should be less than a half LSB voltage of n-bit DAC

$$
V_{r e f} e^{\frac{-t_{o n}}{R_{o n} C_{u}}}<\frac{1}{2} \frac{V_{F S}}{2^{n}}
$$

where $t_{o n} \approx T_{\S} / 2=1 / 2 f_{s}$ is pulse width of clock cycle $T_{s}, V_{r e f}$ is voltage supply for SC, $C_{u}$ is value of unit capacitance. Therefore for assuming $V_{\text {ref }}=V_{F S}$, one of upper bounds of $R_{\text {on }}$ can be found as

$$
R_{\text {on }}<\frac{1}{2 f_{s} C_{u}} \frac{1}{\ln \left(2^{n+1}\right)}
$$




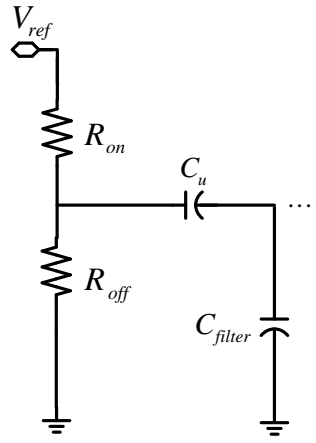

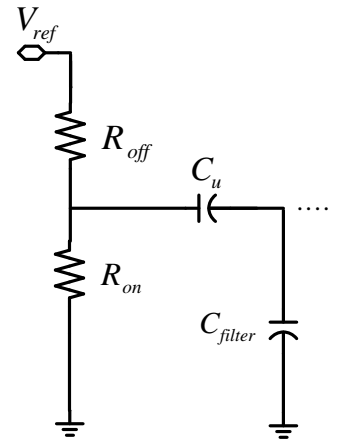

(b)

Figure 6.9: Equivalent circuit model for off-resistance.

For example, with $\mathrm{n}=12, C_{u}=10 \mathrm{fF}, f_{s}=3 \mathrm{GS} / \mathrm{s}, C_{\text {filter }}=2 \mathrm{pF}$, the on-resistance of switches should be less than $1.85 \mathrm{~K} \Omega$.

The circuit model due to off-resistance effect is shown in Fig. 6.9. If data is high, transistor switches to supply $V_{\text {ref }}$ shown in Fig. 6.9a while data is low switch is on to GND shown in Fig. 6.9b. In both cases, the voltage error due to leakage is the same

$$
\Delta V=\frac{R_{o n}}{R_{o n}+R_{o f f}} V_{r e f}
$$

We have to keep this voltage error less than a half LSB of n-bit DAC with the same assumption of $C_{\text {filter }} \gg C_{u}$

$$
\frac{R_{o n}}{R_{o n}+R_{o f f}} V_{r e f}<\frac{1}{2} \frac{V_{F S}}{2^{n}}
$$

From this condition, the lower bound of off-resistance can be obtained

$$
R_{\text {off }}>\left(2^{n+1}-1\right) R_{\text {on }}
$$

However, this voltage error does not cause distortion for DAC output since it has the same effect for all of branches with assuming that all switches have the same $R_{\text {on }}$ and $R_{\text {off }}$. Instead, it reduces output swing due to voltage drop on $R_{\text {on }}$ by leakage current through $R_{\text {off. }}$ Simulation shows that $R_{\text {off }}$ has negligible effect on the linearity of DAC. 


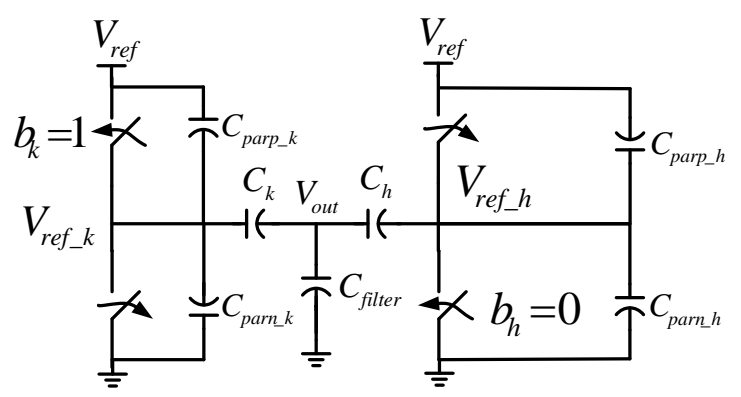

Figure 6.10: Equivalent circuit model for capacitance parasitic.

\subsubsection{Capacitance parasitic effects}

Parasitic capacitances $C_{\text {parp }}$ and $C_{\text {parn }}$ of PMOS and NMOS switches in Fig. 6.10 cause distortion through settling time by adding more capacitances. When $b_{k}=1$, the node at $V_{\text {ref } \_}$is supposed to charge to $V_{\text {ref. }}$. For the worst case we assume voltage at $V_{\text {out }}$ now is zero the total capacitance to be charged is $\left(C_{k}+C_{\text {parn } \_}\right)=\left(C_{u}+C_{p a r}\right)$ for assumption of $C_{\text {parp }}=C_{\text {parn }}=C_{p a r}$. The parasitic will be added to $C_{u}$ in equation (6.32) for the upper bound of $R_{o n}$

$$
R_{o n}<\frac{1}{2 f_{s}\left(C_{u}+C_{p a r}\right)} \frac{1}{\ln \left(2^{n+1}\right)}
$$

The upper bound of $C_{u}$ is calculated from (36) as

$$
C_{u}<\frac{1}{2 f_{s} R_{o n}} \frac{1}{\ln \left(2^{n+1}\right)}-C_{p a r}
$$

\subsection{Switching Noise}

The linearity of high-resolution and high-speed SC DAC is significantly limited by simultaneous switching noise (SSN) caused by the inductance parasitic of bonding wire shown in Fig. 6.11a. The decoupling capacitors (de-caps) $C_{\text {decap }}$ are locally used on-chip for keeping the internal supply stable and have equivalent parasitic resistance $R_{\text {decap }}$. Each supply has two bonds $V_{D D}$ and $V_{S S}$ which can be modelled as $R C L$ [110-113]. Typically, the inductances $L_{D D}$ and $L_{S S}$ are dominant and cause ripple/noise on internal supply since the series resistances $\left(R_{S S}, R_{D D}\right)$ and package capacitances $\left(C_{D D}, C_{S S}\right)$ have less effects. The self-inductances $\left(L_{D D}, L_{S S}\right)$ of a round wire can be estimated as [113] 


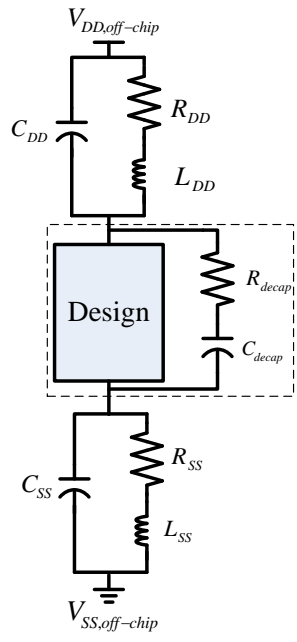

(a)

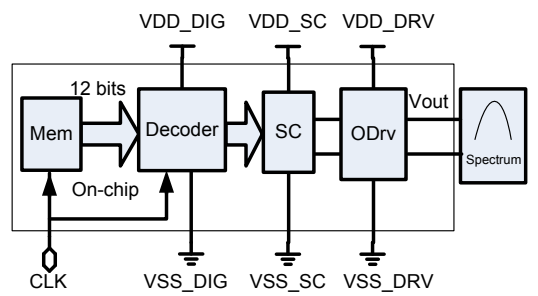

(b)

Figure 6.11: (a) Wire-bonding model. (b) Seprating supplies to reduce noise.

$$
L \approx \frac{\mu_{0} l}{2 \pi}\left[\ln \left(\frac{2 H}{R}\right)-0.75\right]
$$

where $L$ stands for the inductance per unit length, $R$ for radius of the conductor, $H$ for distance from the conductive substrate, and $\mu_{0}$ for permittivity of free space. For typical bond wires, $L$ approximates $1 \mathrm{nH} / \mathrm{mm}$. The maximum SSN voltage is estimated in [111] as

$$
v_{n, \text { max }} \approx \frac{m k L_{S S} \beta_{n}}{2 k t_{r}+2 m(k-1) L_{S S} \beta_{n}} V_{D D}
$$

where $m$ is number of drivers, $\beta_{n}$ is the device transconductance value near the switching threshold voltage $V_{\text {switch }} \approx V_{D D} / 2, \beta_{n} \approx \mu_{n} C_{o x}(W / L)_{n}\left(V_{\text {switch }}-V_{t h}\right)\left[\mathrm{A} / \mathrm{V}^{\text {alpha }}\right]$. For sub-micron MOSFETs, alpha $(\alpha)$ is in the range of $\{1-2\}$. The $t_{r}$ is the rise time. The constant $k=3$ can be used according to [111], the formula (6.39) can be rewritten as

$$
v_{n, \max } \approx \frac{m L_{S S} \beta_{n}}{2 t_{r}+1.3 m L_{S S} \beta_{n}} V_{D D}
$$

There are several ways to reduce SSN. Separating supplies as shown in Fig. 6.11b is one of the ways which was used to avoid noise effect each other with different supplies. The deep-NWell (DNW) layer and guard rings are used to eliminate the body effect and 


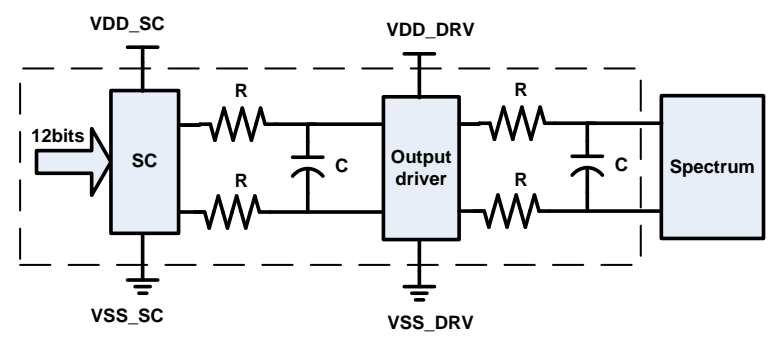

Figure 6.12: Damping/filtering noise on signal paths.

to have more isolation respectively. Based on the voltage error model in (40) SSN can be reduced by minimizing inductance $L$. We have used double bond-wires for all supplies and their effective inductances are lower by only $1 / \sqrt{ } 2$ due to mutual effects. Moreover, the multiple-pads are used to further improve SSN for critical supplies (3 pads for switch-driver, each 2 pads for SC, digital and output driver). Quite big de-caps (MIM cap and MOS cap) have been used to locally suppress noise (700 pF for switchdriver, each $300 \mathrm{pF}$ for SC, digital and output driver). Many parallel de-caps with different values and different self-resonant frequencies (SRF) are soldered on-board to filter out different-frequency noise. Damping resistors were also used to eliminate noise [110-111].

Another damping/filtering technique applied on the signal path were introduced and used in this design as shown in Fig. 6.12. Simulation shows that it can improve around 6 $\mathrm{dB}$ SSN since ripple voltage on signal path is reduced around half. It can be understood from another respective that this filtering technique improves power supply rejection ratio (PSRR) for SC DACs. The low values of $R=10 \Omega, C=600 \mathrm{fF}$ are used to avoid the signal loss which causes reducing the voltage swing of DAC. Since voltage drop on $R$ is negligible and its cut-off frequency is high $\left(f_{C}=53 \mathrm{GHz}\right)$ this technique has no side effect on the output.

The SSN on the supply of switch-driver is the most critical since bigger sizes of switches have been used for fast switching. The upper bound of transistor sizes can be found based on (6.40). This maximum noise voltage should be less than a half LSB of nbit DAC

$$
v_{n, \max }<\frac{1}{2} \frac{V_{F S}}{2^{n}}
$$

From (6.40-6.41), the upper bound of switch sizes can be found

$$
\left(\frac{W}{L}\right)_{n}<\frac{2 t_{r} V_{F S}}{\mu_{n} C_{o x}\left(\frac{V_{D D}}{2}-V_{t h}\right)\left(m L_{S S} V_{D D} 2^{n+1}-1.3 m L_{S S} V_{F S}\right)}
$$




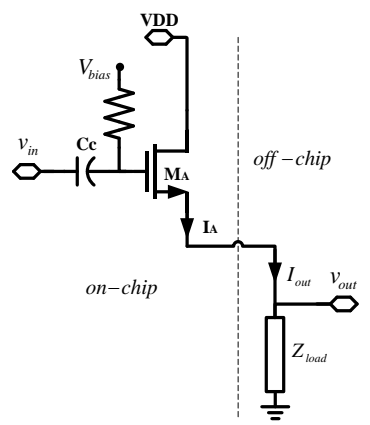

(a)

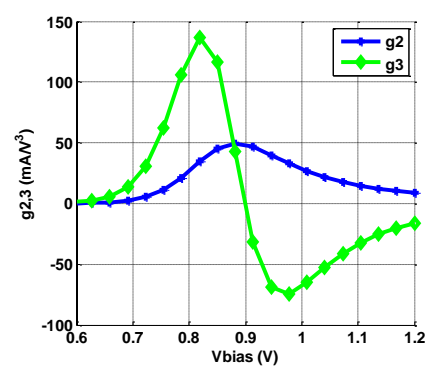

(b)

Figure 6.13: (a) Source-follower buffer. (b) Second and third-order distorion terms of the buffer using ST 65nm CMOS process.

Based on formula (6.42), the transistor sizes of switches in the switch-driver can be chosen depending on $V_{r e f}$, the rise time of clock $t_{r}$, supply $\left(V_{D D}\right)$, the output swing $\left(V_{F S}\right)$, the DAC resolution $(\mathrm{n})$, parasitic inductance of bonding-wires $\left(L_{S S}\right)$ and technology $\left(\mu_{\mathrm{n}} C_{o x}\right)$.

\subsection{Output Driver Design and Analysis}

The output driver is used to drive the off-chip load $(50 \Omega)$ of the capacitive DACs since the SC array does not have the driving capability [61-62]. The driver appears as the most critical circuit causing distortion for capacitive DACs unless the required output voltage swing is low. One solution to this problem is using a closed-loop architecture where the circuit linearity is improved by negative feedback [61]. This technique, however, cannot work well at high frequencies $(>1 \mathrm{GHz})$. Another solution is an open-loop driver that can be supported by interleaving and a pre-distortion technique [62]. However, the latter is usually limited by an assumption of minimum memory effect of the nonlinear object and requires highly precise ADC in the feedback path.

To avoid the complicated calibration and be able to work at high frequency $(\mathrm{GHz}$ range), the open-loop output driver with non-linearity cancellation technique can be used [63]. It shows potential for wire-less application which needs around $300 \mathrm{mV}_{\mathrm{pp}}$ of DAC output to drive mixers. In this design of output driver, we also used open-loop topology and the derivative superposition (DS) technique to achieve high linearity, low power with standard supply of $1.2 \mathrm{~V}$. 


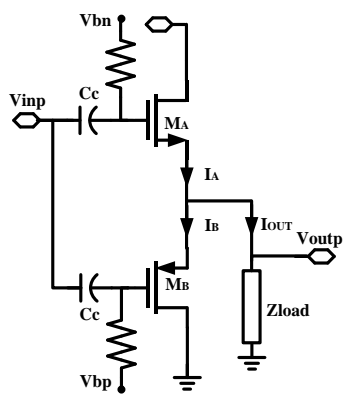

(a)

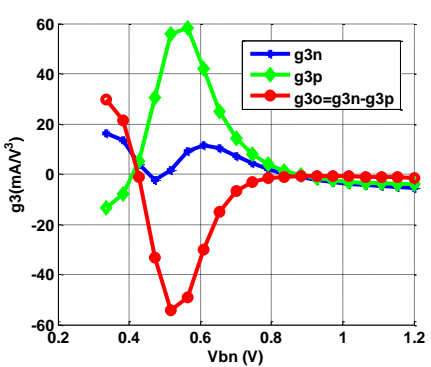

(b)

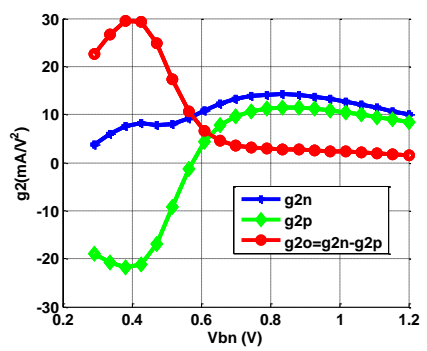

(c)

Figure 6.14: (a) DS technique in SF buffer. (b) Third-order distorion terms of the main transistor (g3n), auxiliary transistor (g3p), and total output (g3). (c) Second-order distorion terms of the main transistor (g2n), auxiliary transistor (g2p), and total output (g2) using ST 65nm CMOS process.

The conventional source-follower (SF) buffer is shown in Fig. 6.13a. The optimal bias for minimizing the third-order trans-conductance $g_{3}$ is very narrow around $V_{\text {bias }}=$ $0.9 \mathrm{~V}$ as shown in Fig. 6.13b. Moreover, the $g_{2}$ is quite high at the same bias point. The derivative superposition (DS) technique introduced in [107] was used to cancel the third-order harmonic distortion for the radio frequency (RF) amplifier. However this proposed output driver achieves the cancellation of both $g_{3}$ and $g_{2}$ at the same input range $(0.8-1.2 \mathrm{~V})$ as shown in Fig. 6.14. 


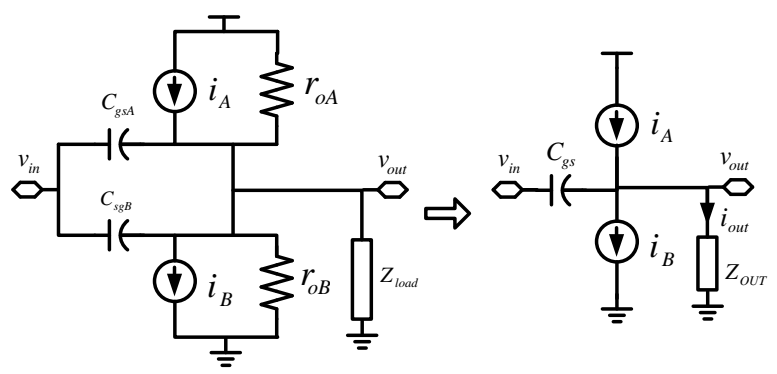

Figure 6.15: Equivalent circuit of the proposed linear output driver.

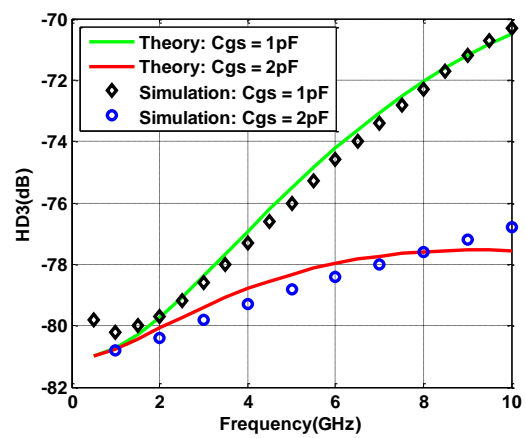

Figure 6.16: HD3 comparison of analytical expressions (6.44) and Spectra RF simulations for the proposed output driver.

\subsubsection{Volterra series model}

The equivalent model of output driver is shown in Fig. 6.15. The parasitic resistances and capacitances $r_{o A}, r_{o B}, C_{g S A}$ and $C_{s g B}$ of the two transistors can be merged as $Z_{\text {out }}=\left(Z_{\text {load }}\left\|r_{o A}\right\| r_{o B}\right)$ and $C_{g s}=C_{g s A}+C_{s g B}$.

The harmonic distortion of output driver can be estimated using the Volterra series model [101]. We focus on the third-order distortion as it prevails over the other distortions

$$
H D 3=\frac{1}{4} \frac{\left|G_{3}\left(j \omega_{1}, j \omega_{1}, j \omega_{1}\right)\right|}{\left|G_{1}\left(j \omega_{1}\right)\right|} A^{2}
$$

where $A$ stands for the amplitude of a sinusoidal signal at the driver input.

Using (6.43) and (A.6.35-A.6.38) of Appendix-G we can calculate HD3 of the output voltage of the proposed circuit as 


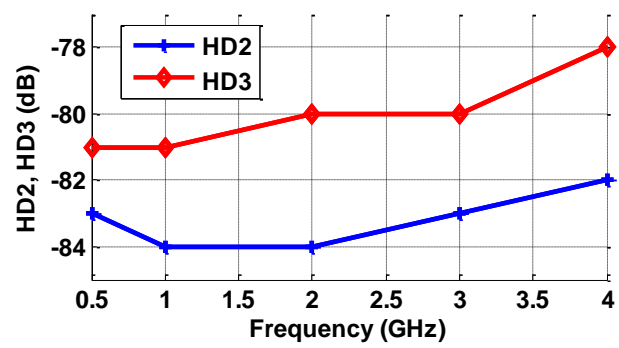

Figure 6.17: HD3 and HD2 versus frequency with 5\% mismatch.

$$
\begin{aligned}
& H D 3=\frac{A^{2}}{4}\left|\frac{Z_{\text {OUT }}\left(\omega_{1}+\omega_{2}+\omega_{3}\right)\left\{\left(g_{3 A}+g_{3 B}\right)\left(1-G_{1}(\omega)\right)^{3}-2\left(g_{2 A}-g_{2 B}\right)\left[G_{2}\left(\omega_{1}+\omega_{2}\right)-\overline{G_{2}\left(\omega_{1}+\omega_{2}\right) G_{1}(\omega)}\right]\right.}{Z_{\text {OUT }}(\omega)\left(g_{1 A}+g_{1 B}+j \omega C_{g s}\right)}\right| \\
& \times\left|\frac{1+Z_{\text {OUT }}(\omega)\left(g_{1 A}+g_{1 B}+j \omega C_{g s}\right)}{1+Z_{\text {OUT }}\left(\omega_{1}+\omega_{2}+\omega_{3}\right)\left[g_{1 A}+g_{1 B}+j\left(\omega_{1}+\omega_{2}+\omega_{3}\right) C_{g s}\right.}\right| \mid
\end{aligned}
$$

The comparisons of HD3 between analytical expressions (6.44) and Spectra RF simulations for the proposed output driver with supply of $1.2 \mathrm{~V}$ are shown in Fig. 6.16. They are quite match with different values of parasitic capacitance $C_{g s}$.

The nominator of second term in (6.44) is supposed to be closed to zero at low frequency in the working bias range in Fig. 6.14b. It is a function of frequency which makes degradation for HD3 at high frequency in Fig. 6.16. Looking at the third term in (6.44), we can see that $C_{g s}$ help to improve HD3 at high frequency because its denominator is proportional with $3 \times \omega$ while the nominator is a function of $1 \times \omega$. This phenomenon can be seen by simulation verification shown in Fig. 6.16. Both curves of analytical and simulated results where $C_{g s}=2 \mathrm{pF}$ is applied are better than the ones where smaller $C_{g s}$ is used.

\subsubsection{Simulation results}

With specification of $300 \mathrm{mV}$ output swing and assuming $5 \%$ between two branches of pseudo-differential circuitry, the output driver is simulated in $65 \mathrm{~nm}$ CMOS technology. Within the band of $0.5-4 \mathrm{GHz}, \mathrm{HD} 2<-82 \mathrm{~dB}$ and $\mathrm{HD} 3<-78 \mathrm{~dB}$ are attained as shown in Fig. 6.17. The dependence of HD2 and HD3 on the bias voltage is demonstrated in Fig. 6.18. HD3 $<-81 \mathrm{~dB}$ and HD2 $<-82 \mathrm{~dB}$ are achieved with the change of bias voltage from $0.85 \mathrm{~V}$ to $1.25 \mathrm{~V}$. The temperature variation effect on the driver linearity is also verified and shown in Fig. 6.19. HD2 is better with higher temperature whereas HD3 is always less than $-80 \mathrm{~dB}$ in the range of $-20-100{ }^{\circ} \mathrm{C}$. 


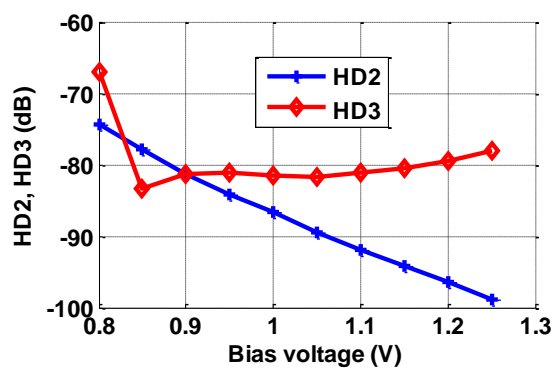

Figure 6.18: HD3 and HD2 versus bias voltage with 5\% mismatch.

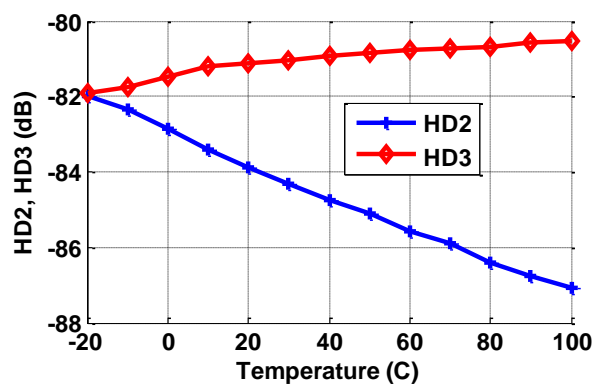

Figure 6.19: HD3 and HD2 versus temperature with 5\% mismatch.

Table 6.3: HD2 and HD3 versus different corners with 5\% mismatch

\begin{tabular}{|c|c|c|}
\hline Corner & HD2 (dB) & HD3 (dB) \\
\hline TT & -84 & -81 \\
\hline FF & -94 & -81 \\
\hline FS & -85 & -80 \\
\hline SF & -96 & -81 \\
\hline SS & -74 & -75 \\
\hline
\end{tabular}

Under different corner of process as shown in Table 6.3, both HD2 and HD3 are better than $-74 \mathrm{~dB}$ which is good enough for driving 12-bit SC DAC.

\subsection{Chip Implementation and Measurement Results}

The measurement setup with on-chip memory for SC DAC testing is shown in Fig. 6.20. A prototype integrated circuit (IC) is fabricated in a standard 65-nm CMOS technology and manually bonded to RF PCB. In order to generate 12-bit data input for full speed testing of the DAC, the 1-Kbit memory has been implemented on-chip [108]. It is written into serially at a low speed $(1 \mathrm{MHz})$ by FPGA and then read at full speed 


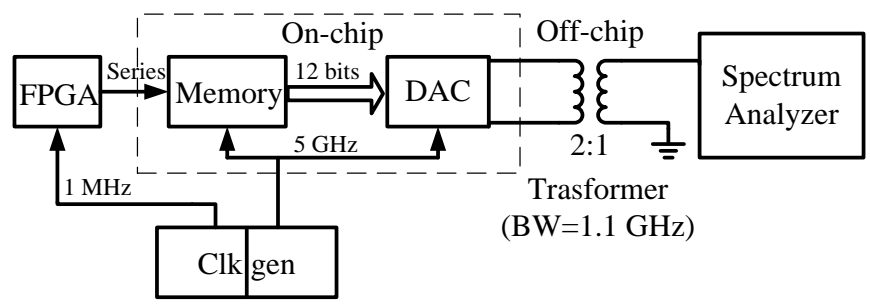

Figure 6.20: Measurement setup of DAC with on-chip memory.

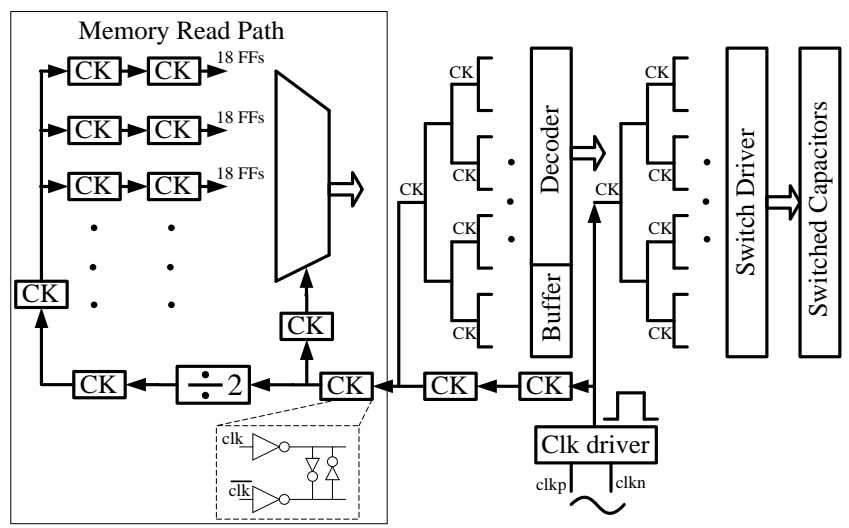

Figure 6.21: Overall clock distribution.

internally during the DAC operation. The 32-bit data is split into two 16-bit streams representing odd and even data, then multiplexed using $f_{s} / 2$ clock to obtain two 8-bit data. However, this design uses only 12 bits of 16 bits. Therefore the memory allows only a 64-point deep signal to be tested and hence the minimum frequency bin spacing in the input signal is $f_{s} / 64$. The sinusoidal differential signal is provided by signal generator and converted to rectangular wave by the internal clock drivers. The strategy of overall clock distribution is shown in Fig. 6.21. The two-channel interleaving of 16 bits uses $f_{s} / 2$ for reading data out. The H-tree topology is used to distribute pseudodifferential clocks for the switch-drivers of switched-capacitor arrays. The total chip occupies $1.2 \times 1.7 \mathrm{~mm}^{2}$ shown in Fig. 6.22. Almost half chip area is occupied by memory and Decaps. The metal-to-metal capacitor is used for both unit and attenuated capacitor since it has better matching feature. Moreover, MIM cap cannot be used since its minimum value is limited by foundry process. In order to reduce parasitic capacitance to the substrate, the bottom plate of $C_{a t t e n}$ is connected towards the output. To have more density layout for saving area, the multi-layers (M2-M3, M3-M4, M4-M5) of metal-tometal caps are used. 


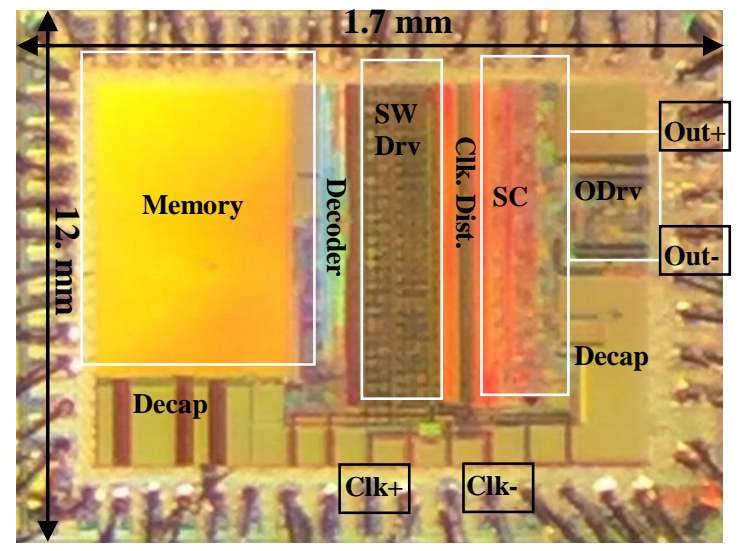

Figure 6.22: Chip photo.

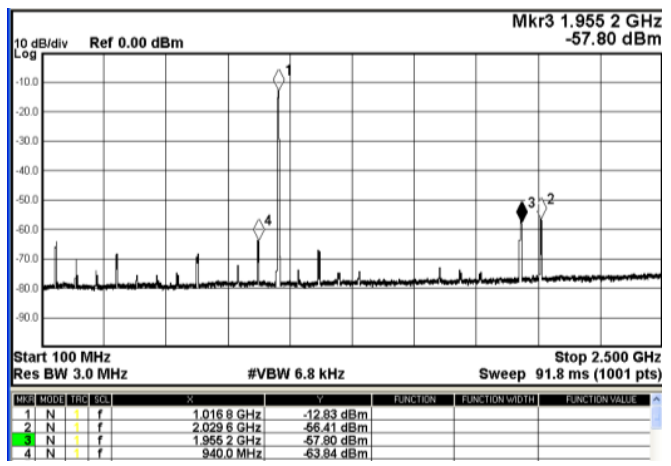

Figure 6.23: Measured $44 \mathrm{~dB}$ SFDR with a $1 \mathrm{GHz}$ single tone at $5 \mathrm{GS} / \mathrm{s}$.

SC DAC can work up to $5 \mathrm{GS} / \mathrm{s}$ for $1 \mathrm{GHz}$ bandwidth (BW) and achieve $44 \mathrm{~dB}$ SFDR as shown in Fig. 6.23. At lower frequency $\left(f_{s}=1 \mathrm{GS} / \mathrm{s}, \mathrm{BW}=16 \mathrm{MHz}\right)$ the better SFDR of $64 \mathrm{~dB}$ is achieved as shown in Fig. 6.24.

As mentioned above, due to a 64-point deep signal to be tested the minimum frequency bin spacing in the input signal is $f_{s} / 64$. Fig. 6.25 shows the measured IM3 of $-38 \mathrm{dBc}$ at $\mathrm{fs}=5 \mathrm{GS} / \mathrm{s}$ for $860 \mathrm{MHz}$ and $1.02 \mathrm{GHz}$ of two tones. The measured SFDR and IM3 versus input frequency at $5 \mathrm{GS} / \mathrm{s}$ are shown in Fig. 6.26. For the bandwidth up to $1 \mathrm{GHz}$ of input signal the SFDR $>44 \mathrm{~dB}$ and IM3 $<-38 \mathrm{dBc}$ are achieved. The SFDR is better than $50 \mathrm{~dB}$ for input frequency up to $800 \mathrm{MHz}$ while IM3 is lower than -50 $\mathrm{dBc}$ for $f_{\text {in }}<700 \mathrm{MHz}$. The total power consumption is $50 \mathrm{~mW}$ from $1 \mathrm{~V}$ digital $(40$ 
Table 6.4: Power and Area Breakdown of the DAC by Function

\begin{tabular}{|l|c|l|c|}
\hline \multicolumn{2}{|c|}{ Power } & \multicolumn{2}{c|}{ Area } \\
\hline \multicolumn{1}{|c|}{ Function } & Power (mW) & Block & Area \\
\hline Decoder & 11 & Decoder & $100 \times 120 \mu \mathrm{m}^{2}$ \\
\hline SW Driver & 14 & SW Driver & $40 \times 700 \mu \mathrm{m}^{2}$ \\
\hline SC & 7 & SC & $140 \times 700 \mu \mathrm{m}^{2}$ \\
\hline Output Driver & 10 & Output Driver & $230 \times 220 \mu \mathrm{m}^{2}$ \\
\hline Clock Distr. & 8 & - & - \\
\hline Total & $\mathbf{5 0}$ & Total & $\mathbf{0 . 1 9} \mathbf{~ m m}^{\mathbf{2}}$ \\
\hline
\end{tabular}

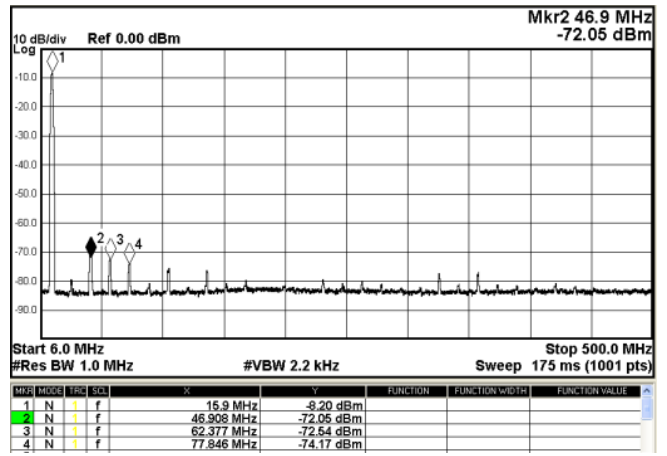

Figure 6.24: Measured $64 \mathrm{~dB}$ SFDR with a $16 \mathrm{MHz}$ single tone at $1 \mathrm{GS} / \mathrm{s}$.

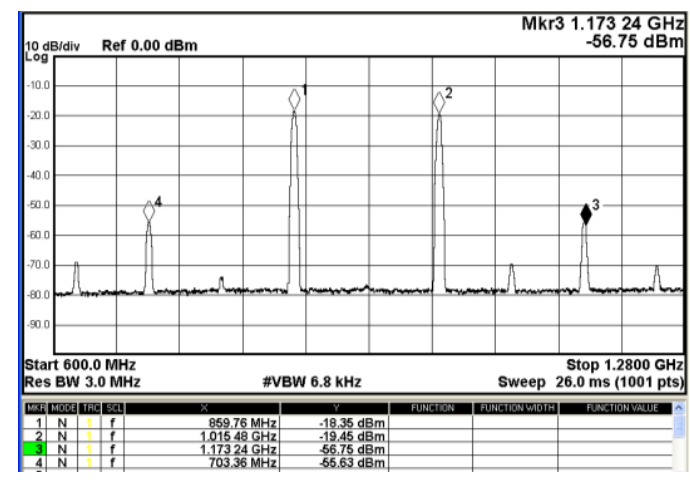

Figure 6.25: Measured IM3 of $-38 \mathrm{dBc}$ with two tones at $5 \mathrm{GS} / \mathrm{s}$.

$\mathrm{mW})$ and $1.2 \mathrm{~V}(10 \mathrm{~mW})$ analog supplies. The power and area breakdown of the SC DAC is shown in Table 6.4.

In order to prove the scalability of this SC DAC for more advanced technology it is measured at lower supply of $0.9 \mathrm{~V}$. The achieved performance of DAC at low supply in 


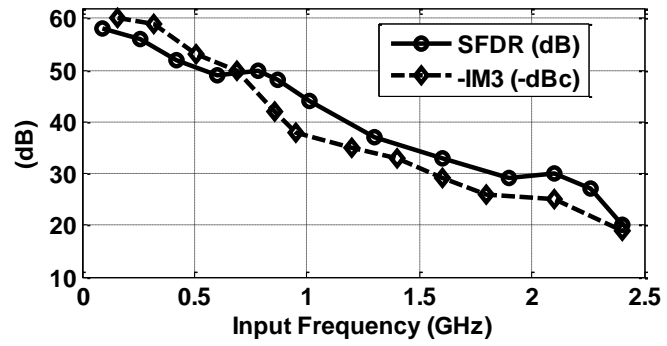

Figure 6.26: Measured SFDR and IM3 versus frequency at 5 GS/s.

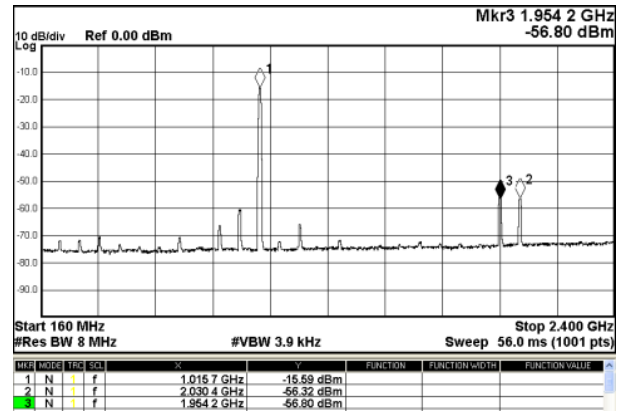

Figure 6.27: Measured $40 \mathrm{~dB}$ SFDR with a $1 \mathrm{GHz}$ single tone at $5 \mathrm{GS} / \mathrm{s}$ with $0.9 \mathrm{~V}$ supply.

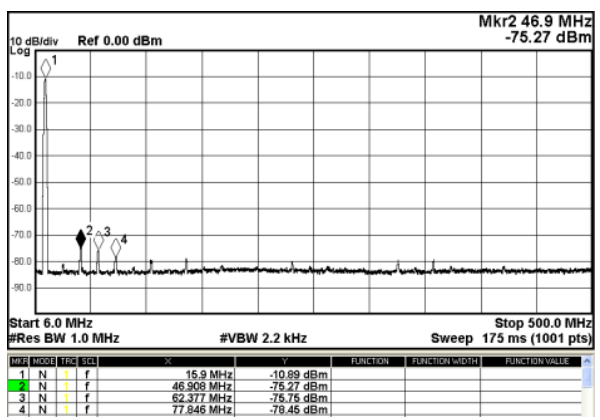

Figure 6.28: Measured $64 \mathrm{~dB}$ SFDR with a $16 \mathrm{MHz}$ single tone at $1 \mathrm{GS} / \mathrm{s}$ with $0.9 \mathrm{~V}$ supply.

terms of SFDR and IM3 are similar to the ones with standard supply of $1.2 \mathrm{~V}$ as shown in Fig. 6.27-6.30. The power dissipation and output swing are lower in this case. It has better scalability than current-steering topology in this respective since the current cells in current-steering DAC need headroom from high supply to have high enough output impedance. Moreover the SC array digitally operates with transistor as switch working in triode region hence gets benefit from scaling technology. 


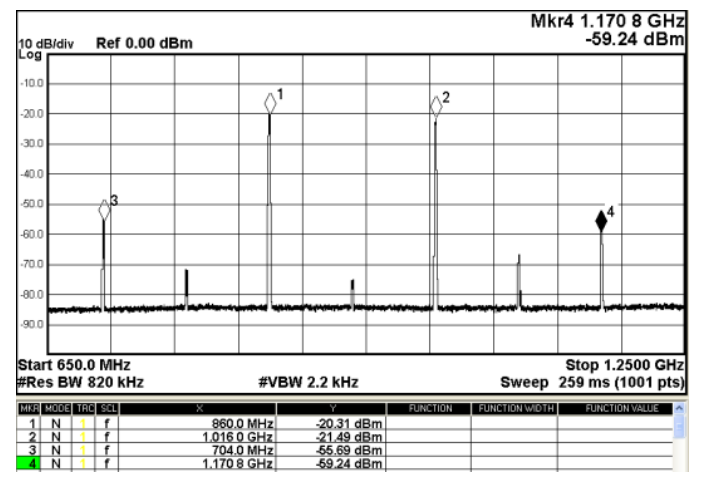

Figure 6.29: Measured IM3 of $-35 \mathrm{dBc}$ with two tones at $5 \mathrm{GS} / \mathrm{s}$ with $0.9 \mathrm{~V}$ supply.

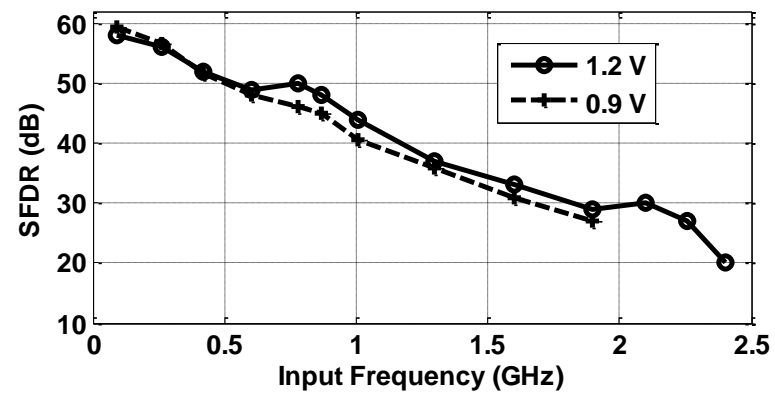

Figure 6.30: Measured SFDR versus frequency at $5 \mathrm{GS} / \mathrm{s}$ with $1.2 \mathrm{~V}$ and $0.9 \mathrm{~V}$ supply.

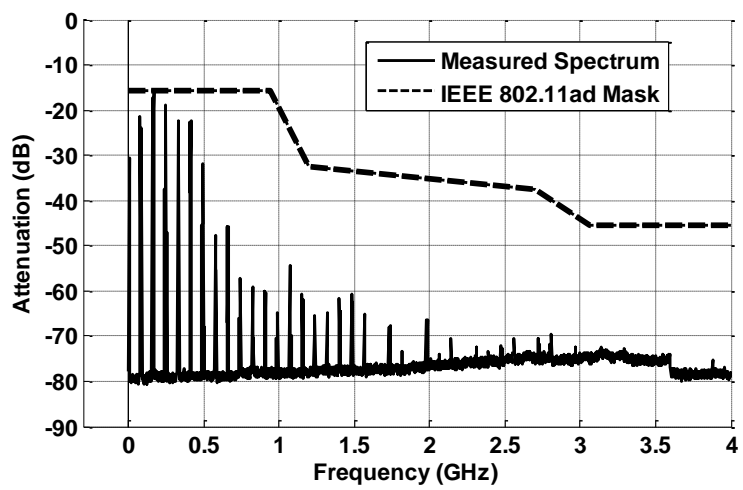

Figure 6.31: Measured spectral mask with 16-QAM single-carrier random data at 5.28 GS/s. 
Since this 12-bit split-segmented SC DAC is designed for $60 \mathrm{GHz}$ radio baseband the spectral mask of 16-QAM modulation scheme with the IEEE 802.11ad (WiGig) standard is verified by measurement. Single-carrier 16-QAM encoded random data with a frequency bin spacing of approximation $80 \mathrm{MHz}$ between 0 to $880 \mathrm{MHz}$ is first generated and pulse-shaped in Matlab with an $18^{\text {th }}$-order root-raised-cosine (RRC) filter having a 0.25 roll-off factor [51]. This data is loaded into the memory for the mask measurement. The filtering is achieved from a combination of the $1.1 \mathrm{GHz}$ interfacing transformer, bonding-wire inductance, pad capacitances and the PCB tracks. It is seen that this overall combination provides a $1.5^{\text {th }}$ order low-pass response between $0.95-1.9$ $\mathrm{GHz}$ and a $2.3^{\text {rd }}$ order low-pass between 1.9-3 GHz. Fig. 6.31 shows the measured spectral mask under these conditions at $5.28 \mathrm{GS} / \mathrm{s}$ operation. It can be observed that the mask of the IEEE 802.11ad (WiGig) standard is met and the out-of-band quantization noise from SC DAC is found not to be a limiting factor.

For comparing to other works, we used the figure-of-merits (FOMs) listed in [55]. There are 5 definitions of FOMs but only two of them are generic i.e. suitable for any kind of DAC type. They are FOM2 and FOM3

$$
\begin{aligned}
& \text { FOM } 2=\frac{2^{N} B W_{N}}{P_{\text {total }}} \\
& \text { FOM } 3=\frac{V_{\text {swing }}}{P_{\text {total }}} B W 10^{\frac{S F D R}{20}}
\end{aligned}
$$

where $N$ is the number of bits, $B W$ (or $B W_{N}$ ) is the signal bandwidth, $P_{\text {total }}$ is the total power consumption, whereas $V_{\text {swing }}$ and $S F D R$ stand for the differential output swing and spurious free dynamic range of DACs, respectively. Table 6.5 shows the comparison of this work with other recent reported DACs which are used for $60-\mathrm{GHz}$ radio baseband. Most of them use current-source topology and no more than 8 bits while this design uses capacitive architecture and 12-bit resolution. The FOM2 and FOM3 of this work are comparable with ones of other designs within the same application. On another hand, for different applications Table 6.6 shows comparison with the recent state-of-the-art reported works. For SC DAC, only 2 designs have recently reported in $[61,62]$. The clock frequencies in those designs are not greater than $800 \mathrm{MHz}$ and signal bandwidth not greater than $400 \mathrm{MHz}$. This work is one of the first 12-bit SC DAC which can work up to $5 \mathrm{GS} / \mathrm{s}$ for signal BW up to $1 \mathrm{GHz}$ to authors' knowledge. The FOMs of this work are calculated at $5 \mathrm{GS} / \mathrm{s}$ for 2 input frequencies $f_{\text {in }}$ of $800 \mathrm{MHz}$ and $1 \mathrm{GHz}$. Its FOM2 is significantly larger than others since the design has 12-bit resolution and low power dissipation while FOM3 is comparable with others for SFDR of (50/44) dB at (800/1000) MHz BW. 
Table 6.5: Comparison with other DACs for the same application of 60-GHz Radio

\begin{tabular}{|c|c|c|c|c|c|c|}
\hline Ref & $\begin{array}{l}\text { This } \\
\text { work }\end{array}$ & $\begin{array}{c}{[51]} \\
\text { CICC09 }\end{array}$ & $\begin{array}{c}{[50]} \\
\text { RFIC12 }\end{array}$ & $\begin{array}{c}{[47]} \\
\text { JSSC13 }\end{array}$ & $\begin{array}{c}{[49]} \\
\text { JSSC13 }\end{array}$ & $\begin{array}{c}{[108]} \\
\text { JSSC15 }\end{array}$ \\
\hline DAC type & SC & $\begin{array}{l}\text { Current } \\
\text { Steering }\end{array}$ & $\begin{array}{l}\text { Current } \\
\text { Steering }\end{array}$ & $\begin{array}{l}\text { Current } \\
\text { Steering }\end{array}$ & $\begin{array}{l}\text { Current } \\
\text { Steering }\end{array}$ & $\Delta \Sigma$ \\
\hline Usage & $\begin{array}{c}\text { 60-GHz } \\
\text { Radio }\end{array}$ & $\begin{array}{c}60-\mathrm{GHz} \\
\text { Radio }\end{array}$ & $\begin{array}{c}60-\mathrm{GHz} \\
\text { Radio }\end{array}$ & $\begin{array}{c}60-\mathrm{GHz} \\
\text { Radio }\end{array}$ & $\begin{array}{c}60-\mathrm{GHz} \\
\text { Radio }\end{array}$ & $\begin{array}{c}\text { 60-GHz } \\
\text { Radio }\end{array}$ \\
\hline Tech. [nm] & 65 & 110 & 40 & 65 & 40 & 65 \\
\hline Supply [V] & 1.2 & $2.5 / 1.2$ & 1.1 & 1.1 & 1.1 & $1.2 / 1$ \\
\hline Fclk [GHz] & 5 & 3.456 & 3.456 & 3.52 & 3.456 & 11 \\
\hline BW [GHz] & $0.8 / 1$ & 0.89 & 0.2 & 0.88 & 0.2 & 1.1 \\
\hline SFDR [dB] & $50 / 44$ & 31 & 40 & 48 & 40 & 53 \\
\hline IM3 [dB] & $-45 /-38$ & - & - & - & - & -49 \\
\hline $\begin{array}{c}\text { Swing } \\
\text { [Vppd] }\end{array}$ & 0.3 & $0.6^{*}$ & $0.6^{*}$ & $0.6^{*}$ & $0.4^{*}$ & 0.5 \\
\hline $\begin{array}{l}\text { Power } \\
{[\mathrm{mW}]}\end{array}$ & $44 / 50$ & 100 & 42 & 71 & 21 & 117 \\
\hline Bits & 12 & 8 & 6 & 7 & 6 & 8 \\
\hline $\begin{array}{c}\text { FOM2 } \\
{\left[10^{12}\right]}\end{array}$ & 75/82 & 2.3 & 0.3 & 1.59 & 0.6 & 39 \\
\hline $\begin{array}{c}\text { FOM3 } \\
{\left[10^{12}\right]}\end{array}$ & $1.7 / 1$ & 0.19 & 0.3 & 1.87 & 0.4 & 2.1 \\
\hline
\end{tabular}

\footnotetext{
${ }^{(*)}$ Estimated.
} 
Table 6.6: Comparison of This Work With State-of-the-art Reported DACs

\begin{tabular}{|c|c|c|c|c|c|c|c|}
\hline Ref & $\begin{array}{l}\text { This } \\
\text { work }\end{array}$ & $\begin{array}{c}{[61]} \\
\text { JSSC } 04\end{array}$ & $\begin{array}{c}{[62]} \\
\text { ASSC10 }\end{array}$ & $\begin{array}{c}{[54]} \\
\text { JSSC } 09\end{array}$ & $\begin{array}{c}{[104]} \\
\text { ISSC } 11\end{array}$ & $\begin{array}{c}{[58]} \\
\text { TVLSI15 }\end{array}$ & $\begin{array}{c}{[59]} \\
\text { JSSC } 15\end{array}$ \\
\hline DAC type & SC & SC & SC & $\begin{array}{l}\text { Current } \\
\text { Steering }\end{array}$ & $\begin{array}{l}\text { Current } \\
\text { Steering }\end{array}$ & $\begin{array}{l}\text { Current } \\
\text { Steering }\end{array}$ & $\begin{array}{l}\text { Current } \\
\text { Steering }\end{array}$ \\
\hline Usage & $\begin{array}{c}\text { 60-GHz } \\
\text { Radio }\end{array}$ & $\begin{array}{l}\text { Comm. } \\
\text { SoC }\end{array}$ & Wireline & Wireline & Wireline & $\begin{array}{c}\text { Comm. } \\
\text { SoC }\end{array}$ & - \\
\hline $\begin{array}{l}\text { Tech. } \\
\text { [nm] }\end{array}$ & 65 & 500 & 90 & 65 & 65 & 28 & 28 \\
\hline$\underset{[V]}{\text { Supply }}$ & 1.2 & 3.3 & $2.5 / 1.2$ & 2.5 & $2.5 / 1.1$ & $2.5 / 1.4$ & 1 \\
\hline $\begin{array}{c}\text { Fclk } \\
{[\text { GHz] }}\end{array}$ & 5 & 0.2 & 0.8 & 2.9 & 56 & 7 & 11 \\
\hline $\begin{array}{c}\text { BW } \\
{[\mathbf{G H z}]}\end{array}$ & $0.8 / 1$ & 0.034 & 0.4 & 0.66 & 26.9 & 1 & 1.1 \\
\hline $\begin{array}{c}\text { SFDR } \\
{[\mathrm{dB}]}\end{array}$ & $50 / 44$ & 70 & 53 & 50 & 30 & 50 & 56 \\
\hline IM3 [dB] & $-45 /-38$ & - & - & -67 & - & -50 & -57 \\
\hline $\begin{array}{c}\text { Swing } \\
\text { [Vppd] }\end{array}$ & 0.3 & 2 & 2.9 & 2.5 & 0.6 & 0.25 & 0.425 \\
\hline $\begin{array}{c}\text { Power } \\
{[\mathrm{mW}]}\end{array}$ & $44 / 50$ & 693 & 103 & 188 & 750 & 145 & $<110$ \\
\hline Bits & 12 & 10 & 12 & 12 & 6 & 6 & 9 \\
\hline $\begin{array}{c}\text { FOM2 } \\
{\left[10^{12}\right]}\end{array}$ & 75/82 & 0.05 & 15.9 & 14.4 & 2.3 & 0.44 & 5.12 \\
\hline $\begin{array}{c}\text { FOM3 } \\
{\left[10^{12}\right]}\end{array}$ & $1.7 / 1$ & 0.3 & 5 & 3.8 & 0.7 & 0.55 & $>2.68$ \\
\hline
\end{tabular}




\section{Chapter 7}

\section{Conclusions and Future Works}

\subsection{Conclusions}

This thesis presents the research conducted on the integrated circuits for analog and mixed signal to solve the key challenges of wideband wireless transceivers at the division of Integrated Circuits and Systems, department of Electrical Engineering, Linköping University, Sweden. Two main parts of research discussed are Part I (Chapter 3, 4): RF receiver front-ends and design for on-chip test on receiver side and Part II (Chapter 5, 6): high speed SC DACs for 60-GHz radio baseband on transmitter side.

The general motivations and applications of RF receiver front-ends including LNAs, DfT and high speed SC DACs are described in Chapter 1. The modern receivers put more demands on bandwidth, noise figure and linearity of LNAs while transmitter 
baseband requires more speed of DACs. Chapter 2 discusses about the design considerations of RF receiver front-ends including RF LNAs, design for on-chip test as well as high speed capacitive DACs.

Chapter 3 presents a tunable receiver front-end with high blocker rejection achieved by two-stage impedance transformation. The front-end design is based on a low noise transconductance amplifier (LNTA) which is well suited to reject out-of-band blockers due to high ratio between its output impedance and on-resistance of switches used in the impedance transformation circuit.

It also presents LNTA design suitable for current-mode wideband front-end in CMOS technology. The amplifier architecture has been derived from the common gate circuit making use of complementary DS technique, which enables highly linear amplification. The tradeoff between the input matching and the transconductance of transistors $\left(g_{m}\right)$ has been mitigated by resistive source degeneration. As a negative feedback it also supported the amplifier linearity. On the other hand, a suitable impedance mismatch at the input was useful to achieve a larger amplifier gain $\left(G_{m}\right)$.

Superior noise performance has been attained by the double capacitive crosscoupling technique in a differential setup as proposed in this work. In effect, the LNTA compares favorably with the state-of-the-art designs both in terms of NF and linearity.

It also presents a complete NF analysis and Volterra series based IIP3 analysis of the amplifier. The obtained estimates were shown compliant with the circuit simulation results. The LNTA was implemented in $65 \mathrm{~nm}$ CMOS as a part of a tunable RF frontend using two-stage $\mathrm{N}$-path filtering technique that provided blocker rejection competitive to SAW filters. Owing to the LNTA design the front-end linearity and noise performance have been placed well in line with the state-of-the-art.

As the complexity of RF integrated circuits is increasing, the task of testing and debugging becomes more and more challenging. The degradation in performance or the drift from the optimal operation points may cause systems to fail. To prevent this effect and ensure quality in terms of process, temperature and voltage variations (PVT), test and calibration of the contemporary RF chips appear to be a must. This approach can be largely facilitated by on-chip techniques (DfT) and in the case of mass production the test cost can be cut as well. RF detector is designed in $65 \mathrm{~nm} \mathrm{CMOS} \mathrm{for} \mathrm{RF} \mathrm{built-in} \mathrm{test}$ system suitable for integrated transceivers is presented in Chapter 4 . The design target is a high sensitivity and high dynamic range over a wide frequency range exploited by the contemporary communication systems. A new RF detector circuit has been proposed using the gain boosting loop and sub-ranging technique. Followed by a high gain VGA and supported by on-chip calibration technique, the detector attains a promising performance, which makes it a good candidate for advanced on-chip measurements. The simulation results are competitive and put this design among the reported state-of-the-art solutions. For a 9-bit T-ADC the obtained in simulation minimum detectable signal is about $-58 \mathrm{dBm}$. This corresponds to the dynamic range of $63 \mathrm{~dB}$ attained in a four sub-range mode with $1.2 \mathrm{~V}$ supply voltage. The relative output error over $0.5 \mathrm{GHz}-9 \mathrm{GHz}$ band is less than $0.8 \%$. 
It also presents an on-chip IP3/IP2 RF test technique suitable for integrated transceivers with embedded RF detectors. The test setup and calibration technique by DC stimuli and statistical regression was briefly described and next, adapted to the IP3/IP2 test. The difference between the detector response to a pure two-tone (used for calibration) and to a two-tone with IMD and HD components was analyzed. We presented an approach to measure IM2/IIP2 using a linear LPF that was also necessary to retrieve the IM3 components from the detector DC response and to calculate IIP3 of the CUT. The proposed technique has been verified by simulation of a practical RF LNA. For this purpose, we have introduced a customized polynomial model of the LNA using Monte-Carlo simulation and statistical regression. The measured IIP3 over the range $-10 \mathrm{dBm}$ to $0 \mathrm{dBm}$ remains within $0.8 \mathrm{~dB}$ compared to PSS and the transient simulation technique in Cadence ${ }^{\circledR}$.

Chapter 5 presents a pipeline SC DAC design in $65 \mathrm{~nm}$ CMOS for high-speed applications. With $300 \mathrm{mVpp}$ output voltage range and $\mathrm{GHz}$ bandwidth the DAC is well suited for the contemporary wideband wireless transmitters. Having designed a highly linear output driver we have found the SC array to limit the DAC performance mostly due to the settling and clock feed-through effect rather than $\mathrm{kT} / \mathrm{C}$ noise and capacitor mismatch. By precise cancelation of the clock feed-through the SFDR was shown to follow well the resolution up to 8 bits for $\mathrm{GHz}$ signal bandwidth ( $45 \mathrm{~dB}$ for $\mathrm{n}=8$ and $\mathrm{BW}=1.36 \mathrm{GHz}$ or $33 \mathrm{~dB}$ for $\mathrm{n}=6$ and $\mathrm{BW}=5.5 \mathrm{GHz}$ ) without extra means of correction. Thereby the tradeoff between DAC bandwidth and resolution accompanied by SFDR has been demonstrated. Higher SFDR can be attained by reducing the clock frequency for increased number of bits and redesigning the SC array accordingly. However, from analysis of the available $(\mathrm{Cu}, \mathrm{W})$ design area we find increasing the resolution from 8 to 9 bits with the corresponding improvement of SFDR, feasible only for clock frequency reduced below $0.5 \mathrm{GHz}$.

The main contribution of this Chapter is showing an alternative way to design highspeed DACs beside current-steering technique which has been widely used. The existing SC DACs have been shown competitive mostly in terms of power consumption for no static current in their SC circuits. Even more advantages can be attained in deep submicron technologies. Using $28 \mathrm{~nm}$ FD SOI for example, the SC circuits with their capacitances and on-resistance switches can achieve better matching than their currentsteering counterparts where the transistors operate in saturation region. The output impedances of current-cells decrease with scaling down due to lower supply, resulting also in more distortion at the DAC output, whereas SC arrays do not have this problem.

Based on the earlier study we have found that the pipeline capacitor array is suffered by clock feed-through effect for high resolution (12-bit) DACs. In order to solve this problem we proposed a 12-bit split-segmented SC DAC array which can work at even higher speed ( $5 \mathrm{GS} / \mathrm{s}$ ), is not limited by kT/C noise, solving clock feed-through problem and no need for multiple-phase clock either as presented in Chapter 6. It describes the design, analysis and implementation of a 12-bit SC split-segmented DAC in 65-nm CMOS. The detail analyses of kT/C noise, capacitor mismatch, settling-time, switching 


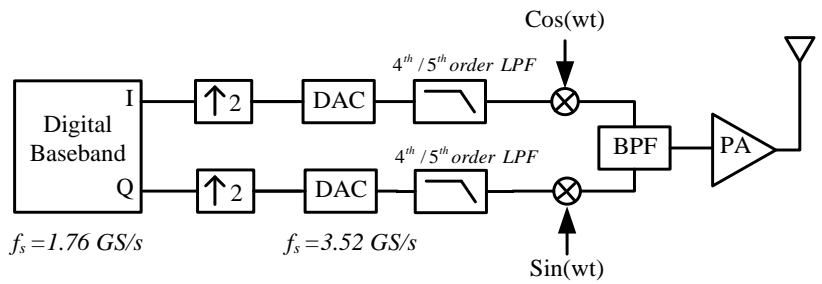

Figure 7.1: Conventional architecture used in [47, 49-50].

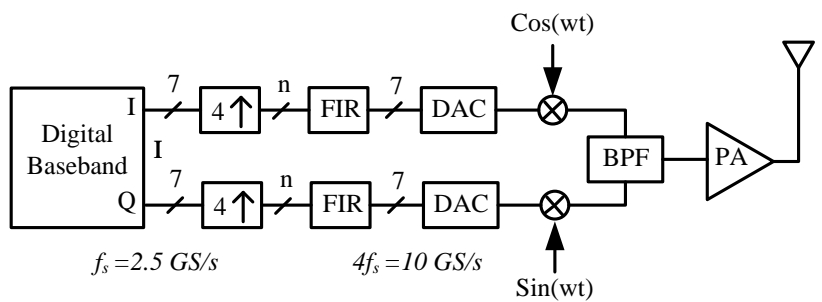

Figure 7.2: High speed Nyquist DAC based architecture proposed in [48].

noise in SC array as well as the linearity of output driver using Volterra Series were described. The SSN is the most critical factor limiting the linearity of SC DACs for high frequency operation. Among capacitive reported DACs, this design achieves highest clock frequency (5 GS/s) and highest BW (1 GHz) with SFDR of $44 \mathrm{~dB}$. The measurement results show that DAC achieves similar linearity (SFDR, IM3) with lower supply $(0.9 \mathrm{~V})$. It means that SC DAC has potential for more advanced technology. Furthermore it has several other advantages such as good capacitor matching and low power. No calibration is needed for the output driver since it has already used the circuit technique for non-linear cancellation. The measured performance puts the design among the recent state-of-the-art reported works. Moreover the spectral mask of the IEEE 802.11ad (WiGig) standard is met therefore this SC DAC is suitable for $60-\mathrm{GHz}$ radio baseband.

\subsection{Future Works}

For Part I of thesis on receiver side, one of the future works can be to implement RF detector and verify the method of IIP2/IIP3 measurement that has been proposed in Chapter 4. Then integration of the designed front-end with design for on-chip test to measure the gains and linearity of each individual RF blocks. Next step would be design of full receiver with baseband circuits. Another future work can be development of receiver front-end to be used for software-defined and cognitive radios which have recently been focused. A software-defined radio (SDR) system is known as a radio 
communication system where components that have been typically implemented in hardware such as amplifiers, mixers, filters, modulators/demodulators and detectors are instead implemented by means of software on a personal computer or embedded system. Significant amounts of signal processing are handed over to the general-purpose processor, rather than being done in special-purpose hardware. Software radios have significant utility for the military and cell phone services, both of which must serve a wide variety of changing radio protocols in real time. On the other hand, cognitive radio (CR) is a transceiver which automatically detects available channels in wireless spectrum and accordingly changes its transmission or reception parameters so more wireless communications may run concurrently in a given spectrum band at a place. This process is also known as dynamic spectrum management. A CR can be aware of its surrounding environment, learns from the environment and adapts its internal states to statistical variations in the incoming RF stimuli by making corresponding changes in certain operating parameters in real time.

For Part II of thesis on transmitter side about high speed capacitive DACs, there are several suggestions for future works. Observing from the design in Chapter 6, it can be seen that SSN is the main factor limiting the performance of high speed SC DACs. To reduce this problem and improve SFDR for DACs we can use 8-bit resolution which is sufficiently good for $60-\mathrm{GHz}$ radio applications. In that way, the number of switch drivers is reduced down to 15 from 63 . Moreover the switch sizes will be 4 times less and making dissipation less on each switch. If reusing the same memory for DAC testing, the 8-bit DAC can utilize all cells of 32x32-memory with full 2 channels to have $f_{s} / 128$ of spacing frequency for multiple-tone measurement.

The headroom-dependent distortion of the proposed SC DAC is completely eliminated when it is implemented without using output driver. This can be realized as DAC is integrated on-chip with LPF/mixer as its load. For the conventional $60-\mathrm{GHz}$ radio transceiver which is used in $[47,49-50]$ as shown in Fig. 7.1, the direct load of DACs is LPFs. The architecture which has been recently introduced for $60-\mathrm{GHz}$ radio in [48] has DAC connected directly to mixer shown in Fig. 7.2. Moreover in superheterodyne transmitter [114], the load of DACs is also LPFs. Since DAC can directly drive the capacitive load (LPFs/mixers) in those cases it does not need the output driver anymore. Removing completely output driver not only helps DAC to reduce dissipation power but also makes SC DAC work as digital based (transistor works as switch). It therefore will definitely have more advantage in advanced technology than current-steering DAC. One of the future works after this thesis would be integrating DAC on-chip into transceiver to have complete $60-\mathrm{GHz}$ radio system.

This SC array can also used in $\triangle \sum \mathrm{DAC}[108]$ where the digital modulator is connected to m-bit SC DAC array instead of current-source cells as shown in Fig. 7.3. The motivation for it is making the performance of the complete $\triangle \sum \mathrm{DAC}$ not to depend on headroom voltage through output impedance of current cells. When implementing $\Delta \sum \mathrm{SC}$-DAC on-chip with the real load of LPF/mixer into the transceiver system it will also show the potential of scalability. 


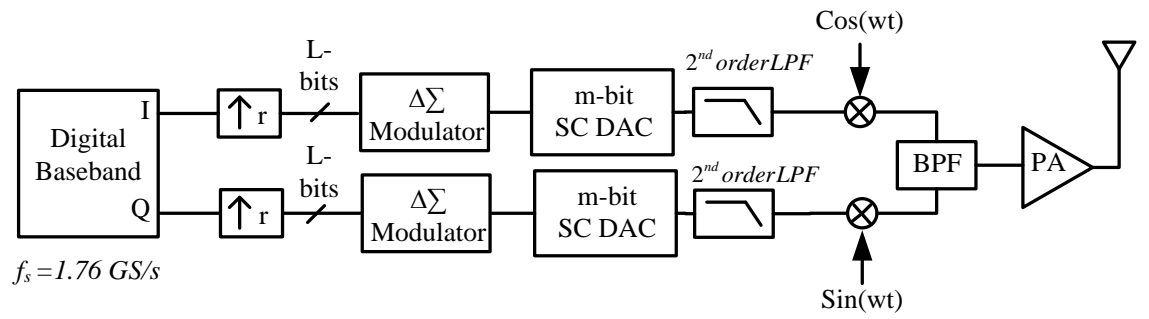

Figure 7.3: $\triangle \sum$ DAC proposed in [108] can use SC DAC cells instead of current-steering ones. 


\section{Appendix}

\section{PART I: LNA}

APPENDIX A

DERIVATION OF THE VOLTERRA OPERATORS FOR THE PROPOSED LNTA: G1, G2 AND G3

For the circuit shown in Fig. 3.12 the respective currents and voltages can be expressed as

$$
\begin{gathered}
v_{s g p}=\frac{-n_{p} v_{\text {in }}-R_{s p}\left(i_{d p}-\frac{v_{\text {outn }}}{r_{d p}}\right)}{m_{p}} \\
v_{g s n}=\frac{n_{n} v_{\text {in }}-R_{\text {sn }}\left(i_{d n}+\frac{v_{\text {outn }}}{r_{d n}}\right)}{m_{n}} \\
v_{s p}=v_{s g p}+\frac{v_{\text {in }}}{2}, v_{s n}=\frac{v_{\text {in }}}{2}-v_{g s n}
\end{gathered}
$$




$$
v_{\text {outn }}=k_{\text {loadn }}\left(i_{d p}-i_{d n}+\frac{v_{s p}}{r_{d p}}+\frac{v_{s n}}{r_{d n}}\right)
$$

where

$$
\begin{gathered}
m_{p}=1+R_{s p}\left(C_{g s p} s+\frac{1}{r_{d p}}\right), m_{n}=1+R_{s n}\left(C_{g s n} s+\frac{1}{r_{d n}}\right) \\
n_{p}=1+\frac{R_{s p}}{2 r_{d p}}, n_{n}=1+\frac{R_{s n}}{2 r_{d n}} \\
k_{p}=\frac{g_{1 p} R_{s p}}{m_{p}}, k_{n}=\frac{g_{1 n} R_{s n}}{m_{n}} \\
\hat{Z}_{L}=\frac{Z_{L}}{1+Z_{L}\left(\frac{1}{r_{d p}}+\frac{1}{r_{d n}}\right)}
\end{gathered}
$$

Substituting $(3.23,3.24)$ into (A.3.1, A.3.2) with maximum $3^{\text {rd }}$ order of $v_{i n}$, we have

$$
\begin{gathered}
v_{s g p}^{3}=\frac{B_{p}^{3}}{m_{p}^{3}\left(1+k_{p}\right)^{3}}, v_{g s n}^{3}=\frac{B_{n}^{3}}{m_{n}^{3}\left(1+k_{n}\right)^{3}} \\
v_{s g p}^{2}=\frac{B_{p}^{2}}{m_{p}^{2}\left(1+k_{p}\right)^{2}}\left(1-\frac{2 R_{s p} g_{2 p} B_{p}}{m_{p}^{2}\left(1+k_{p}\right)^{2}}\right) \\
v_{s g p}=\frac{1}{m_{p}\left(1+k_{p}\right)}\left\{B_{p}^{2}-\frac{B_{n}^{2}}{m_{n}^{2}\left(1+k_{n}\right)^{2}}\left(1-\frac{2 R_{s p} g_{2 n} B_{n}}{m_{p}^{2}\left(1+k_{p}\right)^{2}}\right)\right. \\
\left.v_{g s n}=\frac{1}{m_{n}\left(1+k_{n}\right)}\left\{1-\frac{2 R_{s p} g_{2 p} B_{p}}{m_{p}^{2}\left(1+k_{p}\right)^{2}}\right)-\frac{R_{s p} g_{3 p} B_{p}^{3}}{m_{p}^{3}\left(1+k_{p}\right)^{3}}\right\} \\
\left.B_{n}-\frac{R_{s n} g_{2 n} B_{n}^{2}}{m_{n}^{2}\left(1+k_{n}\right)^{2}}\left(1-\frac{2 R_{s n} g_{2 n} B_{n}}{m_{n}^{2}\left(1+k_{n}\right)^{2}}\right)-\frac{R_{s n} g_{3 n} B_{n}^{3}}{m_{n}^{3}\left(1+k_{n}\right)^{3}}\right\}
\end{gathered}
$$

where

$$
B_{p}=-n_{p} v_{i n}+\frac{R_{s p}}{r_{d p}} v_{\text {outn }}, \quad B_{n}=n_{n} v_{\text {in }}-\frac{R_{s n}}{r_{d n}} v_{\text {outn }}
$$

Substituting (3.23, 3.24), (A.3.3, A.3.9-A.3.13) into (A.3.4), we have 


$$
\begin{aligned}
& v_{\text {out }}=\frac{\hat{Z}_{L} B_{p}\left(g_{1 p}+\frac{1}{r_{d p}}\right)}{\left(1+k_{p}\right) m_{p}}+\frac{\hat{Z}_{L} B_{p}^{2}}{m_{p}^{2}\left(1+k_{p}\right)^{3}}\left\{g_{2 p}\left(1-\frac{2 R_{s p} g_{2 p} B_{p}}{m_{p}^{2}\left(1+k_{p}\right)^{2}}\right)+\frac{g_{3 p} B_{p}}{m_{p}\left(1+k_{p}\right)}\right\} \\
& -\frac{\hat{Z}_{L} B_{n}\left(g_{1 n}+\frac{1}{r_{d n}}\right)}{\left(1+k_{n}\right) m_{n}}-\frac{\hat{Z}_{L} B_{n}^{2}}{m_{n}^{2}\left(1+k_{n}\right)^{3}}\left\{g_{2 n}\left(1-\frac{2 R_{s n} g_{2 n} B_{n}}{m_{n}^{2}\left(1+k_{n}\right)^{2}}\right)+\frac{g_{3 n} B_{n}}{m_{n}\left(1+k_{n}\right)}\right\}
\end{aligned}
$$

\section{APPENDIX B}

DERIVATION OF THE VOLTERRA OPERATORS FOR THE PROPOSED LNTA: A1, A2, A3, $\mathrm{H} 1, \mathrm{H} 2$ AND $\mathrm{H} 3$

For the circuit of Fig. 3.12 the current and voltage equations follow

$$
\begin{gathered}
v_{i n}=v_{s o}-2 R_{s o} i_{s o} \\
i_{s o}=-s C_{g s p} v_{s g p 1}+s C_{g s n} v_{g s n 1}+v_{i n p}\left(\frac{1}{s L_{p}}+\frac{1}{s L_{n}}\right)+i_{d p 2}-i_{d n 2} \\
+s C_{g s p} v_{s g p 2}-s C_{g s n} v_{g s n 2}+\frac{v_{s p 2}-v_{\text {outp }}}{r_{d p}}-\frac{v_{\text {outp }}-v_{s n 2}}{r_{d n}}
\end{gathered}
$$

where

$$
\begin{gathered}
v_{s g p 1}=v_{s g p}\left(v_{\text {in }}, v_{\text {out }}\right), v_{s g p 2}=v_{s g p}\left(-v_{\text {in }},-v_{\text {out }}\right) \\
v_{g s n 1}=v_{g s n}\left(v_{\text {in }}, v_{\text {out }}\right), v_{g s n 2}=v_{g s n}\left(-v_{\text {in }},-v_{\text {out }}\right) \\
v_{s p 2}=v_{s p}\left(-v_{\text {in }},-v_{\text {out }}\right), v_{s n 2}=v_{s n}\left(-v_{\text {in }},-v_{\text {out }}\right) \\
i_{d p 2}=i_{d p}\left(-v_{\text {in }},-v_{\text {out }}\right), i_{d n 2}=i_{d n}\left(-v_{\text {in }},-v_{\text {out }}\right)
\end{gathered}
$$

For differential mode, the single voltages should be

$$
v_{\text {inp }}=-v_{\text {inn }}=\frac{v_{\text {in }}}{2}, v_{\text {outp }}=-v_{\text {outn }}=\frac{v_{\text {out }}}{2}
$$




\section{Part II: Pipeline Capacitive dacs}

APPENDIX C

Derivation of THE Volterra Operators For THE Proposed Driver AT LOW FREQUENCY

For the circuit shown in Fig. 5.13(a) the respective currents and voltages can be expressed as

$$
\begin{gathered}
i_{A}=g_{1 A} v_{g s}+g_{2 A} v_{g s}{ }^{2}+g_{3 A} v_{g s}{ }^{3} \\
i_{B}=g_{1 B} v_{g s}+g_{2 B} v_{g s}{ }^{2}+g_{3 B} v_{g s}{ }^{3} \\
i_{\text {OUT }}=i_{A}+i_{B} \\
v_{g s}=v_{\text {in }}-v_{\text {OUT }} \\
v_{\text {OUT }}=R_{S} i_{\text {OUT }}
\end{gathered}
$$

Where $g_{i A}$ and $g_{i B}$ are the $i^{t h}$-order coefficients of MA and MB, accordingly, obtained by taking the derivative of the drain dc current $I_{D S}$ with respect to the gate-source voltage $V_{G S}$ at the dc bias point

$$
\begin{gathered}
g_{1 A}=\frac{\partial I_{D S A}}{\partial V_{G S A}}, g_{1 B}=\frac{\partial I_{D S B}}{\partial V_{G S B}}, g_{1}=g_{1 A}+g_{1 B} \\
g_{2 A}=\frac{1}{2 !} \frac{\partial^{2} I_{D S A}}{\partial V_{G S A}^{2}}, g_{2 B}=\frac{1}{2 !} \frac{\partial^{2} I_{D S B}}{\partial V_{G S B}^{2}}, g_{2}=g_{2 A}+g_{2 B} \\
g_{3 A}=\frac{1}{3 !} \frac{\partial^{3} I_{D S A}}{\partial V_{G S A}^{3}}, g_{3 B}=\frac{1}{3 !} \frac{\partial^{3} I_{D S B}}{\partial V_{G S B}^{3}}, g_{3}=g_{3 A}+g_{3 B}
\end{gathered}
$$

Applying the Volterra series to the output voltage

$$
v_{\text {oUT }}=G_{1} \circ v_{i t}+G_{2} \circ v_{i t}^{2}+G_{3} \circ v_{i n}^{3}
$$

Substituting (A.5.1-A.5.4) into (A.5.5), we have

$$
\begin{aligned}
& v_{\text {OUT }}=R_{S}\left(g_{1 A}+g_{1 B}\right)\left(v_{i n}-v_{\text {OUT }}\right) \\
& +R_{S}\left(g_{2 A}+g_{2 B}\right)\left(v_{i n}-v_{\text {OUT }}\right)^{2}+R_{S}\left(g_{3 A}+g_{3 B}\right)\left(v_{i n}-v_{\text {OUT }}\right)^{3}
\end{aligned}
$$

Then from (A.5.9) and (A.5.10) the functions $G_{1}, G_{2}$, and $G_{3}$ are obtained respectively

$$
G_{1}=\frac{R_{S}\left(g_{1 A}+g_{1 B}\right)}{1+R_{S}\left(g_{1 A}+g_{1 B}\right)}
$$




$$
\begin{gathered}
G_{2}=\frac{R_{S}\left(g_{2 A}+g_{2 B}\right)}{\left[1+R_{S}\left(g_{1 A}+g_{1 B}\right)\right]^{3}} \\
G_{3}=\frac{R_{S}\left(g_{3 A}+g_{3 B}\right)}{\left[1+R_{S}\left(g_{1 A}+g_{1 B}\right)\right]^{4}}-\frac{2\left[R_{S}\left(g_{2 A}+g_{2 B}\right)\right]^{2}}{\left[1+R_{S}\left(g_{1 A}+g_{1 B}\right)\right]^{5}}
\end{gathered}
$$

In a similar way, for the DS-only driver we find

$$
\begin{aligned}
& G_{1}=R_{S}\left(g_{1 A}+g_{1 B}\right) \\
& G_{2}=R_{S}\left(g_{2 A}+g_{2 B}\right) \\
& G_{3}=R_{S}\left(g_{3 A}+g_{3 B}\right)
\end{aligned}
$$

APPENDIX D

Derivation OF THE Volterra OPERATORS FOR THE PROPOSED DRIVER AT HIGH FREQUENCY

For the circuit of Fig. 5.16 the current and voltage equations follow

$$
\begin{gathered}
i_{A}=g_{1 A} v_{g s}+g_{2 A} v_{g s}{ }^{2}+g_{3 A} v_{g s}{ }^{3} \\
i_{B}=g_{1 B} v_{g s}+g_{2 B} v_{g s}{ }^{2}+g_{3 B} v_{g s}{ }^{3} \\
i_{\text {OUT }}=i_{A}+i_{B}+\left(v_{i n}-v_{\text {OUT }}\right) s C_{g s} \\
v_{g s}=v_{i n}-v_{\text {OUT }} \\
v_{\text {OUT }}=Z_{\text {OUT }} i_{\text {OUT }}
\end{gathered}
$$

For the Volterra series applied to the output voltage

$$
v_{\text {OUT }}=G_{1} \circ v_{i n}+G_{2} \circ v_{i n}^{2}+G_{3} \circ v_{i n}^{3}
$$

Substituting (A.5.17-A.5.20) into (A.5.21), we have

$$
\begin{aligned}
& v_{\text {OUT }}=Z_{\text {OUT }}\left(g_{1 A}+g_{1 B}+s C_{g s}\right)\left(v_{\text {in }}-v_{\text {OUT }}\right) \\
& +Z_{\text {OUT }}\left(g_{2 A}+g_{2 B}\right)\left(v_{\text {in }}-v_{\text {OUT }}\right)^{2} \\
& +Z_{\text {OUT }}\left(g_{3 A}+g_{3 B}\right)\left(v_{\text {in }}-v_{\text {OUT }}\right)^{3}
\end{aligned}
$$

Next, using (A.5.22) and (A.5.23) the Volterra transfer functions $G_{1}, G_{2}$, and $G_{3}$ are obtained 


$$
\begin{aligned}
& G_{1}(\omega)=\frac{Z_{\text {OUT }}(\omega)\left(g_{1 A}+g_{1 B}+j \omega C_{g s}\right)}{1+Z_{\text {OUT }}(\omega)\left(g_{1 A}+g_{1 B}+j \omega C_{g s}\right)} \\
& G_{2}\left(\omega_{1}+\omega_{2}\right)=\frac{Z_{\text {OUT }}\left(\omega_{1}+\omega_{2}\right)\left(g_{2 A}+g_{2 B}\right)\left(1-G_{1}(\omega)\right)^{2}}{\left[1+Z_{\text {OUT }}\left(\omega_{1}+\omega_{2}\right)\left(g_{1 A}+g_{1 B}+j\left(\omega_{1}+\omega_{2}\right) C_{g s}\right)\right]^{3}} \\
& G_{3}\left(\omega_{1}+\omega_{2}+\omega_{3}\right)= \\
& \frac{Z_{\text {OUT }}\left(\omega_{1}+\omega_{2}+\omega_{3}\right)\left(g_{3 A}+g_{3 B}\right)\left(1-G_{1}(\omega)\right)^{3}}{1+Z_{\text {OUT }}\left(\omega_{1}+\omega_{2}+\omega_{3}\right)\left[g_{1 A}+g_{1 B}+j\left(\omega_{1}+\omega_{2}+\omega_{3}\right) C_{g s}\right]} \\
& -\frac{2 Z_{\text {OUT }}\left(\omega_{1}+\omega_{2}+\omega_{3}\right)\left(g_{2 A}+g_{2 B}\right)\left[G_{2}\left(\omega_{1}+\omega_{2}\right)-\overline{G_{2}\left(\omega_{1}+\omega_{2}\right) G_{1}(\omega)}\right]}{1+Z_{\text {OUT }}\left(\omega_{1}+\omega_{2}+\omega_{3}\right)\left[g_{1 A}+g_{1 B}+j\left(\omega_{1}+\omega_{2}+\omega_{3}\right) C_{g s}\right]}
\end{aligned}
$$

where

$$
\begin{aligned}
& \overline{G_{2}\left(\omega_{1}+\omega_{2}\right) G_{1}(\omega)}= \\
& \frac{\left[G_{2}\left(\omega_{1}+\omega_{2}\right) G_{1}\left(\omega_{3}\right)+G_{2}\left(\omega_{1}+\omega_{3}\right) G_{1}\left(\omega_{2}\right)+G_{2}\left(\omega_{3}+\omega_{2}\right) G_{1}\left(\omega_{1}\right)\right]}{3}
\end{aligned}
$$

\section{APPENDIX E}

SIMULATION RESULTS FROM 28 NM CMOS FD SOI TENOLOGY

A more advanced technology such as $28 \mathrm{~nm}$ CMOS FD SOI is used to show advantages of SC pipeline DAC due to scaling and other features available in this case. Using this technology the design areas demonstrated in Chapter $\mathrm{V}$ are becoming larger for the same clock frequency and resolution. This can be seen from comparison of the transistor width range shown in Fig. 5.8 and Fig. A.5.1, where the latter shows $\left[W_{\min }, W_{\max }\right]=[63,3000] \mu \mathrm{m}$. However, the real advantage is that higher frequencies and resolutions can be attained with this technology.

In FD SOI technology the oxide capacitance $C_{o x}$ is much smaller than in standard bulk CMOS so $C_{g}(W)$ is smaller and the channel length $L=30 \mathrm{~nm}$ results in less $R_{\text {on }}$ as well. That is why the upper bounds for $C_{u}$ are higher than the ones obtained with $65 \mathrm{~nm}$ technology. A smaller value of $C_{o x}$ also results in smaller $C_{p}(W)$ in (5.20) which makes the $C_{u}$ lower bound (due to feed-through effect) more relaxed as well.

In particular, at $3 \mathrm{GHz}$ clock frequency as demonstrated in Fig. A.5.2 it is possible to design 11-bit SC DAC using $\left(W, C_{u}\right)=(125 \mu \mathrm{m}, 500 \mathrm{fF})$ with a sufficient design area around to guarantee circuit robustness. The value of unit capacitance is larger than required by $\mathrm{kT} / \mathrm{C}$ noise condition (14) $C_{u}>50 \mathrm{fF}$ for $n=11$. Observe that the lower $C_{u}$ 
bound is, in fact, dictated by the feedthrough condition (5.20) (green line) and not by $R_{\text {off }}$ in (5.31). It also shows that 12-bit DAC at $3 \mathrm{GHz}$ is not feasible (green dotted line) unless the demands for accuracy are relaxed to one LSB.

Additionally, in Fig. A.5.3 we show the speed capability of a 9-bit SC DAC that with a tight design area demonstrates a theoretical data rate of $135 \mathrm{Gbps}$.

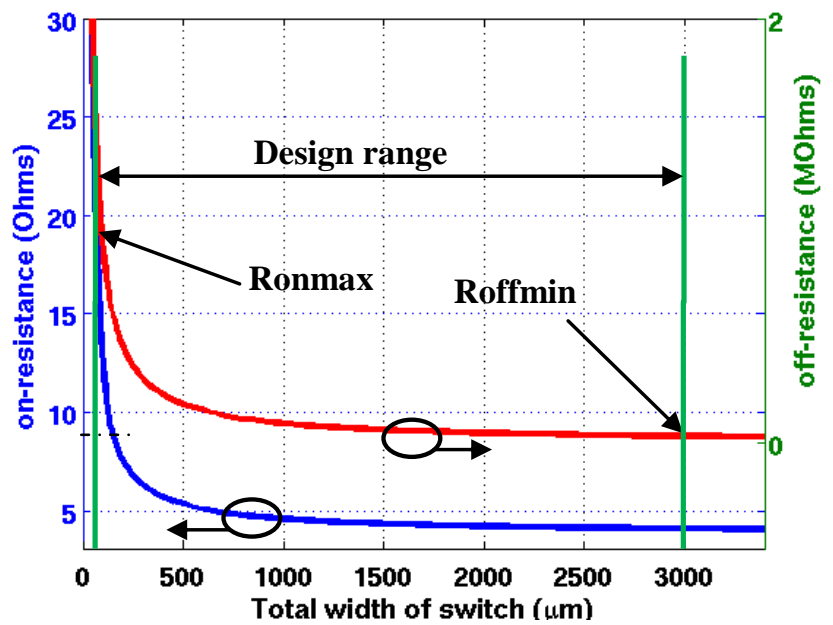

Figure A.5.1: Off-resistance and on-resistance of T-gate switch vs. width in $28 \mathrm{~nm}$ CMOS FD SOI with $L=30 \mathrm{~nm}\left(W_{p} / W_{n}=3\right)$.

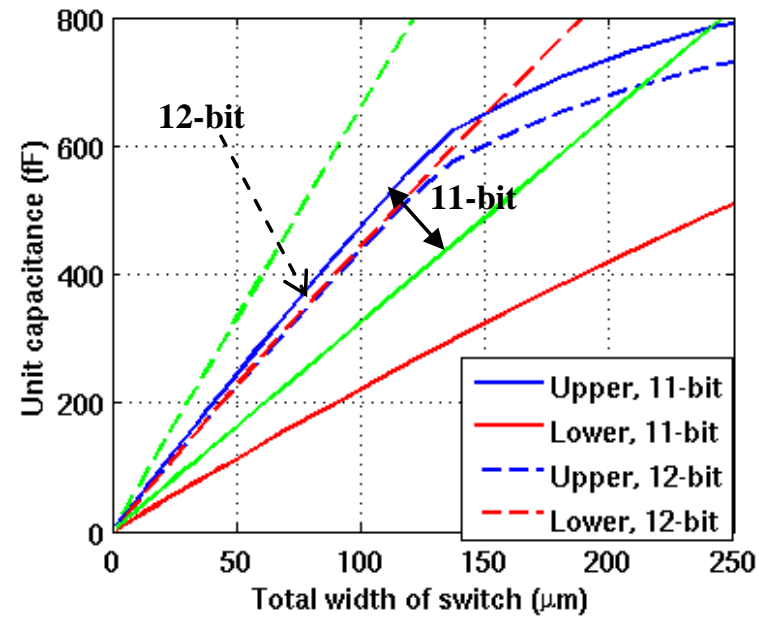

Figure A.5.2: Design area $\mathrm{W}-\mathrm{C}_{\mathrm{u}}$ for 28nm CMOS FD SOI, 11-bit and 12-bit SC DAC assuming half LSB accuracy, $f_{S}=3 \mathrm{GHz}, K_{d i f}=20 \%$ and $K_{\text {comp }}=1 \%$. 


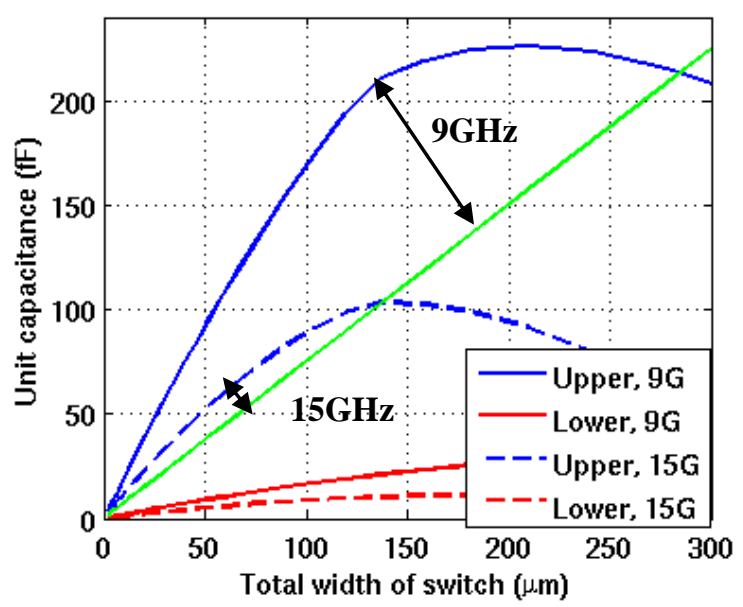

Figure A.5.3: Design area W-C $\mathrm{C}_{\mathrm{u}}$ for 28nm CMOS FD SOI, 9-bit SC DAC with different clock frequencies $\left(f_{s}\right), K_{\text {dif }}=20 \%, K_{\text {comp }}=1 \%$.

\section{PART III: SEgMented SC DACS}

\section{APPENDIX F}

DERIVATION OF THE SC-ARRAY NOISE ANALYSIS

From Fig. 6.3, impedance of each binary branch is described as

$$
Z_{b 1}(i)=\frac{R_{o n}}{2^{i}}+\frac{1}{j \omega C_{u} 2^{i}}
$$

The total admittance of the second binary branches is

$$
Y_{b 1}=\sum_{i=0}^{n-m-k-1} \frac{1}{Z_{b 1}(i)}
$$


The total impedance of the second binary branches and $C_{\text {atten } 2}$ is found

$$
Z_{b 1 \text { atten } 2}=\frac{1}{Y_{b 1}}+\frac{1}{j \omega C_{a t t e n 2}}
$$

The total admittance of the first, second binary branches and $C_{\text {atten } 2}$ is

$$
Y_{b 1 \text { atten } 2}=\frac{1}{Z_{b 1 \text { atten } 2}}+\sum_{i=0}^{n-m-k-1} \frac{1}{Z_{b 1}(i)}
$$

The total impedance of the first, second binary branches and $C_{\text {atten } 1}, C_{a t t e n 2}$ is

$$
Z_{\text {b1atten } 1}=\frac{1}{Y_{b 1 a t t e n 2}}+\frac{1}{j \omega C_{\text {atten } 1}}
$$

Impedance of each thermometer branch is as

$$
Z_{t h 1}=R_{o n}+\frac{1}{j \omega C_{u}}
$$

The total admittance of all thermometer branches is as

$$
Y_{t h 1}=\frac{2^{m}-2}{Z_{t h 1}}
$$

From Fig. 6.4, impedance of each binary branch as

$$
Z_{b 2}(p)=\frac{R_{o n}}{2^{p}}+\frac{1}{j \omega C_{u} 2^{p}}
$$

The total admittance of the second binary branches as

$$
Y_{b 2}=\sum_{p=0}^{n-m-k-1} \frac{1}{Z_{b 2}(p)}
$$

The total impedance of the second binary branches and $C_{\text {atten } 2}$

$$
Z_{b 2 a t t e n 2}=\frac{1}{Y_{b 2}}+\frac{1}{j \omega C_{a t t e n 2}}
$$

The total admittance of the first binary branches 


$$
Y_{b 2 a}=\sum_{j=0, j \# i}^{n-m-k-1} \frac{1}{Z_{b 2}(j)}
$$

The total impedance of the first binary branches

$$
Z_{b 2 a}=\frac{1}{Y_{b 2 a}}
$$

Impedance of each thermometer branch as

$$
Z_{t h 2}=R_{o n}+\frac{1}{j \omega C_{u}}
$$

The total admittance of all thermometer branches as

$$
Y_{t h 2}=\frac{2^{m}-1}{Z_{t h 2}}
$$

The total admittance of thermometer and load $\left(C_{\text {filter }}\right)$ as

$$
Y_{2 l o a d}=Y_{\text {th } 2}+j \omega C_{\text {filter }}
$$

From Fig. 6.5, impedance of each binary branch as

$$
Z_{b 3}(p)=\frac{R_{o n}}{2^{p}}+\frac{1}{j \omega C_{u} 2^{p}}
$$

The total admittance of the second binary segment as

$$
Y_{b 32}=\sum_{p=0, p \# i}^{n-m-k-1} \frac{1}{Z_{b 3}(p)}
$$

The total impedance of the second binary segment as

$$
Z_{b 32}=\frac{1}{Y_{b 32}}
$$

The total admittance of the first binary segment as

$$
Y_{b 31}=\sum_{p=0}^{n-m-k-1} \frac{1}{Z_{b 3}(p)}
$$


The total impedance of the first binary segment as

$$
Z_{b 31}=\frac{1}{Y_{b 31}}
$$

Impedance of each thermometer branch as

$$
Z_{t h 3}=R_{o n}+\frac{1}{j \omega C_{u}}
$$

The total admittance of all thermometer branches as

$$
Y_{t h 3}=\frac{2^{m}-1}{Z_{t h 3}}
$$

The total admittance of thermometer and load $\left(C_{\text {filter }}\right)$ as

$$
Y_{3 l o a d}=Y_{\text {th } 3}+j \omega C_{f i l t e r}
$$

\section{APPENDIX G}

DERIVATION OF THE VOLTERRA OPERATORS FOR THE PROPOSED OUTPUT DRIVER

For the equivalent circuit shown in Fig. 6.15 the current and voltage equations follow

$$
\begin{gathered}
i_{A}=g_{1 A} v_{g s A}+g_{2 A} v_{g s A}^{2}+g_{3 A} v_{g s A}{ }^{3} \\
i_{B}=g_{1 B} v_{s g B}+g_{2 B} v_{s g B}^{2}+g_{3 B} v_{s g B}{ }^{3} \\
i_{\text {OUT }}=i_{A}-i_{B}+\left(v_{\text {in }}-v_{\text {OUT }}\right) s C_{g s} \\
v_{g s A}=v_{\text {in }}-v_{\text {OUT }} \\
v_{\text {SgB }}=v_{\text {OUT }}-v_{\text {in }} \\
v_{\text {OUT }}=Z_{\text {OUT }} i_{\text {OUT }}
\end{gathered}
$$

where $g_{i A}$ and $g_{i B}$ are the $i^{\text {th }}$-order coefficients of $\mathrm{M}_{\mathrm{A}}$ and $\mathrm{M}_{\mathrm{B}}$, accordingly, obtained by taking the derivative of the drain dc current $I_{D S}$ with respect to the gate-source voltage $V_{G S}$ at the dc bias point

$$
g_{1 A}=\frac{\partial I_{D S A}}{\partial V_{G S A}}, g_{1 B}=\frac{\partial I_{S D B}}{\partial V_{S G B}}
$$




$$
\begin{aligned}
& g_{2 A}=\frac{1}{2 !} \frac{\partial^{2} I_{D S A}}{\partial V_{G S A}^{2}}, g_{2 B}=\frac{1}{2 !} \frac{\partial^{2} I_{S D B}}{\partial V_{S G B}^{2}} \\
& g_{3 A}=\frac{1}{3 !} \frac{\partial^{3} I_{D S A}}{\partial V_{G S A}^{3}}, g_{3 B}=\frac{1}{3 !} \frac{\partial^{3} I_{S D B}}{\partial V_{S G B}^{3}}
\end{aligned}
$$

Applying the Volterra series to the output voltage

$$
v_{\text {oUT }}=G_{1} \circ v_{i n}+G_{2} \circ v_{i n}^{2}+G_{3} \circ v_{i t}^{3}
$$

Substituting (A.6.24-A.6.28) into (A.6.29), we have

$$
\begin{aligned}
& v_{\text {OUT }}=Z_{\text {OUT }}\left(g_{1 A}+g_{1 B}+s C_{g s}\right)\left(v_{\text {in }}-v_{\text {OUT }}\right) \\
& +Z_{\text {OUT }}\left(g_{2 A}-g_{2 B}\right)\left(v_{\text {in }}-v_{\text {OUT }}\right)^{2} \\
& +Z_{\text {OUT }}\left(g_{3 A}+g_{3 B}\right)\left(v_{\text {in }}-v_{\text {OUT }}\right)^{3}
\end{aligned}
$$

Next, using (A.6.33) and (A.6.34) the Volterra transfer functions $G_{1}, G_{2}$, and $G_{3}$ are obtained

$$
\begin{gathered}
G_{1}(\omega)=\frac{Z_{\text {OUT }}(\omega)\left(g_{1 A}+g_{1 B}+j \omega C_{g s}\right)}{1+Z_{\text {OUT }}(\omega)\left(g_{1 A}+g_{1 B}+j \omega C_{g s}\right)} \\
G_{2}\left(\omega_{1}+\omega_{2}\right)=\frac{Z_{\text {OUT }}\left(\omega_{1}+\omega_{2}\right)\left(g_{2 A}-g_{2 B}\right)\left(1-G_{1}(\omega)\right)^{2}}{\left[1+Z_{\text {OUT }}\left(\omega_{1}+\omega_{2}\right)\left(g_{1 A}+g_{1 B}+j\left(\omega_{1}+\omega_{2}\right) C_{g s}\right)\right]} \\
G_{3}\left(\omega_{1}+\omega_{2}+\omega_{3}\right)= \\
\frac{Z_{\text {OUT }}\left(\omega_{1}+\omega_{2}+\omega_{3}\right)\left(g_{3 A}+g_{3 B}\right)\left(1-G_{1}(\omega)\right)^{3}}{1+Z_{O U T}\left(\omega_{1}+\omega_{2}+\omega_{3}\right)\left[g_{1 A}+g_{1 B}+j\left(\omega_{1}+\omega_{2}+\omega_{3}\right) C_{g s}\right]} \\
-\frac{2 Z_{\text {OUT }}\left(\omega_{1}+\omega_{2}+\omega_{3}\right)\left(g_{2 A}-g_{2 B}\right)\left[G_{2}\left(\omega_{1}+\omega_{2}\right)-\overline{G_{2}\left(\omega_{1}+\omega_{2}\right) G_{1}(\omega)}\right]}{1+Z_{O U T}\left(\omega_{1}+\omega_{2}+\omega_{3}\right)\left[g_{1 A}+g_{1 B}+j\left(\omega_{1}+\omega_{2}+\omega_{3}\right) C_{g s}\right]}
\end{gathered}
$$

where

$$
\begin{aligned}
& \overline{G_{2}\left(\omega_{1}+\omega_{2}\right) G_{1}(\omega)}= \\
& \frac{\left[G_{2}\left(\omega_{1}+\omega_{2}\right) G_{1}\left(\omega_{3}\right)+G_{2}\left(\omega_{1}+\omega_{3}\right) G_{1}\left(\omega_{2}\right)+G_{2}\left(\omega_{3}+\omega_{2}\right) G_{1}\left(\omega_{1}\right)\right]}{3}
\end{aligned}
$$




\section{References}

[1] L.E. Franks and I.W. Sandberg, "An alternative approach to the realizations of network functions: N-path filter,” Bell Syst. Tech. J., pp.1321-1350, 1960.

[2] B. Cook, A. Berny, A. Molnar et al. "Low-Power $2.4 \mathrm{GHz}$ Transceiver with passive Rx front-end and $400 \mathrm{mV}$ supply," IEEE J. Solid-State Circuits, vol. 41, no. 12, pp. 2757-2766, Dec. 2006.

[3] A.E.E. Qualkadi, M.E. Kaamouchi, J. M. Paillot, D. et al., "Fully integrated highQ switched capacitor bandpass filter with center frequency and bandwidth tuning," in IEEE Symp. Radio Freq. Integr. Circuits, pp. 681-684, 2007.

[4] A. Mirzaei, H. Darabi, J. Leete, et al. "Analysis and optimization of currentdriven passive mixers in narrowband direct-conversion receivers," IEEE J. SolidState Circuits, vol. 44, no.10, pp. 2678-2688, Oct. 2009.

[5] A. Mirzaei, H. Darabi, J. Leete, and Y. Chang, "Analysis and optimization of direct-conversion receivers with $25 \%$ duty-cycle current-driven passive mixers," IEEE Trans. Circuits Syst. I, Reg. Papers, vol. 57, no.9, pp. 2353-2366, Sep. 2010. 
[6] C. Andrews and A. Molnar, "A passive mixer-first receiver with digitally controlled and widely tunable RF interface," IEEE J. Solid-State Circuits, vol. 45, no. 12, pp. 2696-2708, Dec. 2010.

[7] C. Andrews and A. Molnar, "Implications of passive mixer transparency for impedance matching and noise figure in passive mixer-first receivers," IEEE Trans. Circuits Syst. I, Reg. Papers, vol. 57, no. 12, pp. 3092-3103, Dec. 2010.

[8] A. Ghaffari, E. Klumperink, M. Soer, and B. Nauta, "Tunable high-Q N-path band-pass filters: Modeling and verification," IEEE J. Solid-State Circuits, vol. 46, no. 5, pp. 998-1010, 2011.

[9] A. Mirzaei and H. Darabi, "Analysis of imperfections on performance of 4-phase passive-mixer-based high-Q bandpass filters in SAW-less receivers," IEEE Trans. Circuits Syst. I, Reg. Papers, vol. 58, no. 5,pp. 879-892, May 2011.

[10] A. Mirzaei and H. Darabi, A. Yazdi, et al., "A $65 \mathrm{~nm}$ CMOS quad-band SAWless receiver SoC for GSM/GPRS/EDGE," IEEE J. Solid-State Circuits, vol. 46, no. 4, pp. 950-964, 2011.

[11] Z. Ru, et al. "Digitally enhanced software-defined radio receiver robust to out-ofband interference," J. of Solid-State Circuits, vol. 44, no. 12, December 2009.

[12] C.Y. Yu, et al., "A SAW-Less GSM/GPRS/EDGE Receiver Embedded in 65-nm SoC," IEEE J. Solid-State Circuits, vol. 46, iss. 12, pp. 3047-4060, Dec. 2011.

[13] D. Murphy, H. Darabi, et al. "A blocker-tolerant, noise-cancelling receiver suitable for wideband wireless applications," IEEE J. Solid-State Cir., vol. 47, no. 12, pp. 2943-2963, Dec. 2012.

[14] J. Kim and J. Silva-Martinez, "Low-Power, Low-Cost CMOS Direct-Conversion Receiver Front-End for Multistandard Applications," IEEE J. of Solid-State Circuits, vol. 48, no. 9, pp. 2090-2103, 2013.

[15] I. Fabiano t al., "SAW-Less Analog Front-End Receivers for TDD and FDD," IEEE J. of Solid-State Circuits, vol. 48, no.12, pp. 3067-3079, 2013.

[16] F. Qazi, et al.,"Two-stage highly selective receiver front-end based on impedance transformation filtering," IEEE, Trans. on Circuit and Systems II, vol. 62, no. 5, pp. 421-425, 2015. 
[17] L.E. Franks, and I.W. Sandberg, "An Alternative Approach to the Realization of Network Transfer Functions: The N-Path Filter," Bell System Technical Journal, vol. 39, no. 5, pp. 1321-1350, 1960.

[18] H. Zhang and E.Sanchez-Sinencio "Linearization Techniques for CMOS Low Noise Amplifiers: A Tutorial," Transactions on Circuit and Systems, vol. 58, no. 1, pp.22-36, January 2011.

[19] V. Aparin et al., "Linearization of CMOS LNAs via optimum gate biasing," Proc. IEEE Int. Conf. Circuits and Systems, pp. 748-751, 2004.

[20] T.W. Kim, B. Kim, and K Lee, "Highly linear receiver front-end adopting MOSFET transconductance linearization by multiple gated transistors," $J$. of Solid-State Circuits, vol.39, No.1, pp. 223-229, 2004.

[21] V. Aparin et al., "Modified derivative suprposition method for linearizing FET low-noise amplifiers," IEEE Trans. Microwave Theory Tech., vol. 53, no. 2, pp. 571-581, 2005.

[22] D. Im, et al., "A wideband CMOS low noise Amplifier Employing Noise and IM2 distortion cancellation for a digital TV tuner," J. of Solid-State Circuits, vol. 44, no. 3, pp. 686-698, Mar. 2009.

[23] W-H. Chen, et al., "A highly linear broadband CMOS LNA employing noise and distortion cancellation," J. of Solid-State Circuits, vol. 43, no. 5, May 2008.

[24] H.M. Geddada, et al., "Wide-Band Inductorless Low-Noise Trans-conductance Amplifiers With High Large-Signal Linearity," IEEE Trans. on Microwave Theory and Techniques, vol. 62, no. 7, pp. 1495-1505, 2014.

[25] N. Ahsan, et al., "Highly linear wideband low power current mode LNA," Intl. Conf. on Systems and Electronic Systems, ICSES, 4 pp. 2008.

[26] Q-T. Duong and J. Dąbrowski, "Low noise transconductance amplifier design for continuous-time delta sigma wideband front-end," in Europ. Conf. on Cir. Theory and Design. (ECCTD), pp. 825 - 828, 2011.

[27] H. Zhang, et al., "A low-power, linearized, ultra-wideband LNA design technique," J. of Solid-State Circuits, vol. 44, no. 2, pp.320-330, Feb 2009.

[28] W.Zhuo, et al., "Using capacitive cross-coupling technique in RF low noise amplifier and down-conversion mixer design," European Solid-State Circuits Conference, ESSCIRC, pp.77-80, 2000. 
[29] F. Bruccoleri, et al., "Wide-band CMOS low-noise amplifier exploiting thermal noise canceling," J. of Solid-State Circuits, vol. 39, no. 2, pp. 275-282, Feb. 2004.

[30] M. Reiha and J. Long "A 1.2V Reactive-Feedback 3.1-10.6 GHz Low-Noise Amplifier in 0.13um CMOS," J. of Solid State Circuits, vol. 42, no. 5, pp. 10231033, May 2007.

[31] A. Amer, et al., "A 90-nm Wideband Merged CMOS LNA and Mixer Exploiting noise cancellation," J. of Solid-State Circuits, vol. 42, no 2, December 2007.

[32] S. Woo et al., "A 3.6mW differential common-gate CMOS LNA with positivenegative feedback," IEEE International Solid-State Circuits Conference - Digest of Technical Papers, ISSCC, pp. 218-219, 2009.

[33] J. Kim et al., "Wideband Common-Gate CMOS LNA Employing Dual Negative Feedback With Simultaneous Noise, Gain, and Bandwidth Optimization," IEEE Trans. on Microwave Theory and Techniques, vol. 58, no. 9, pp. 2340 2351, 2010.

[34] S-C. Blaakmeer, et al., "Wideband balun-LNA with simultaneous output balancing, noise-canceling and distortion-canceling," J. of Solid-State Circuits, vol. 43, no. 6, pp. 1341-1350, June 2008 .

[35] F. Jonsson, et al.,"RF detector for on-chip amplitude measurements," Electronics Letters, vol. 40, no. 20, pp. 1239-1240, Sept. 2004.

[36] V. Acharya, et al. "On-chip RMS Detector using CMOS Quad for RF testing," Silicon Monolithic Integr. Cir. in RF Systems, pp. 1-4, 2009.

[37] A. Valdes-Garcia, et al. "A CMOS RF RMS Detector for Built-in Testing of Wireless Transceiver,” VLSI Test Symposium, pp. 249-254, 2005.

[38] A. Valdes-Garcia, et al. "A Broadband CMOS Amplitude Detector for On-chip RF Measurements," Trans. on Instr. and Measurement, vol. 57, no. 7, pp. 14701477, 2008.

[39] R. Ramzan, J. Dabrowski, "CMOS RF/DC Voltage Detector for on-chip Test," IEEE INMIC, pp. 472-476, 2006.

[40] Q. Wang, M. Soma. "RF Front-end System Gain and Linearity Built-in Test," VLSI Test Symposium, pp. 227-233, 2006. 
[41] S. B. Sleiman, M. Ismail, "A CMOS Amplitude Detector for RF-BIST and Calibration,” ICECS, pp. 807-810, 2009.

[42] S. B. Sleiman, M. Ismail, "Transceiver Parameter Detection using a High Conversion Gain RF Amplitude Detector,” ISCAS, pp. 2059-2062, 2010.

[43] R. Ramzan, J. Dabrowski. "On-chip Calibration of RF Detectors by DC Stimuli and Artificial Neural Networks," Radio Frequency Integrated Circuits Symposium, pp. 571-574, 2008.

[44] IEEE, “IEEE 802.11ad - Amendment 3: Enhancements for Very High Throughput in the 60 Ghz Band," Standard, Mar. 2012. (http://ieeexplore.ieee.org/servlet/opac?punumber=6171797)

[45] "High rate $60 \mathrm{GHz}$ PHY, MAC and PALs : ECMA-387," Standard, Dec. 2010. (http://www.ecma-international.org/)

[46] "WirelessHD Specification v1.1 overview," Standard, May 2010. (http://www.wirelesshd.org/)

[47] N. Saito, et al., “ A fully integrated 60-GHz CMOS transceiver chipset based on WiGig/IEEE 802.11ad with built-in self calibration for mobile usage," IEEE J. Solid-State Circuits, vol. 48, no. 12, pp. 3146-3159, Dec. 2013.

[48] J. Muller, et al., “ A 7-bit $18^{\text {th }}$ order 9.6 GS/s FIR up-sampling filter for high data rate $60-\mathrm{GHz}$ wireless transmitters," IEEE J. Solid-State Circuits, vol. 47, no. 7, pp. 1743-1756, July. 2012.

[49] K. Okada, et al.," A full 4-channel $6.3 \mathrm{~Gb} / \mathrm{s} 60 \mathrm{GHz}$ directconversion transceiver with low-power analog and digital baseband circuitry," IEEE JSSC, vol. 48, no. 1, pp. 46-65, Jan. 2013,.

[50] M. Miyahara, et al., “ An $84 \mathrm{~mW} 0.36 \mathrm{~mm}^{2}$ analog baseband circuits for $60 \mathrm{GHz}$ wireless transceiver in $40 \mathrm{~nm}$ CMOS," IEEE RFIC, June 2012, pp. 495-498.

[51] M. Tokumaru, et al., "A $14.6^{\text {th }}$-order $3.456 \mathrm{GHz}$ transmit baseband filter in 110 nm CMOS for milimeter-wave communication systems," IEEE CICC, pp. 175178, 2009.

[52] G. A. M. V. D. Plas, et al.,"A 14-bit intrinsic accuracy $\mathrm{Q}^{2}$ random walk CMOS DAC,” IEEE J. Solid-State Circuits, vol. 34, no. 12, pp. 1708-1718, Mar. 1999. 
[53] X. Wu, et al., “A 130nm CMOS 6-bit Full Nyquist 3 GS/s DAC,” IEEE J. SolidState Circuits, vol. 43, pp. 2396-2403, Nov. 2008.

[54] C. H. Lin, et al.,"A 12 bit 2.9 GS/s DAC with IM3 < -60 dBc Beyond $1 \mathrm{GHz}$ in 65nm CMOS,” IEEE J. Solid-State Circuits, vol. 44, pp. 3285-3293, Dec. 2009.

[55] P. Palmers, et al.,"A 10-Bit 1.6-GS/s 27-mW Current-Steering D/A Converter With 550-MHz 54-dB SFDR Bandwidth in 130-nm CMOS," IEEE TCASI, vol. 57, pp. 2870-2879, Nov. 2010.

[56] Wei-Te Lin, et al., "A 12-bit $40 \mathrm{~nm}$ DAC achieving SFDR > $70 \mathrm{~dB}$ at $1.6 \mathrm{GS} / \mathrm{s}$ and IMD <-61 dB at $2.8 \mathrm{GS} / \mathrm{s}$ with DEMDRZ technique," IEEE J. S.S. Circuits, vol. 49, pp. 708-717, Mar. 2014.

[57] Shiyu Su, et al., “A 12 bit 1 GS/s Dual-Rate Hybrid DAC with an 8 GS/s unrolled pipeline delta-sigma modulator achieving > 75 dB SFDR over the Nyquist Band," IEEE J. S.S. Circuits, vol. 50, pp. 896-907, Apr. 2015.

[58] Georgi I. Radulov, et al.,"A 28-nm CMOS 7-GS/s 6-bit DAC with dft clock and memory reaching SDFR > $50 \mathrm{~dB}$ up to $1 \mathrm{GHz}$," IEEE Trans. VLSI, vol. 49, pp. 1941-1945, Sep. 2015.

[59] Erik Olieman, et al.,"An interleaved full Nyquist high-speed DAC technique," IEEE J. S.S. Circuits, vol. 50, pp. 704-713, Mar. 2015.

[60] Franco Maloberti, Data Converters. Springer, 2008.

[61] G. Manganaro, et al.,"A Dual 10-b 200-MSPS Pipeline D/A Converter With DLL-Based Clock Synthesizer," IEEE J. Solid-State Circuits, vol. 39, pp. 18291838, Nov. 2004.

[62] C. Daigle, et al.,"A 12-bit 800-MS/s Switched-Capacitor DAC with Open-loop Output Driver and Digital Predistortion," IEEE Asian Solid-State Circuits Conference, pp. 1-4, Nov. 2010.

[63] Quoc-Tai Duong, et al.," Design and Analysis of High Speed Capacitive Pipeline DACs," Journal of Analog Integrated Circuits and Signal Processing, vol. 80, no. 3, pp. 359-374, Sept. 2014.

[64] B. Razavi, “RF microelectronics," second edition, New York, 2012.

[65] B. Razavi, "Impact of distributed gate resistance on the performance of MOS Devices," IEEE TCASI, vol. 41, pp. 750-754, Nov. 1994. 
[66] B. Razavi, “Design of Analog CMOS Integrated Circuits,” Boston, 2001.

[67] Quoc-Tai Duong, et al.,“A 1-GHz Bandwidth 12-bit SC DAC for 60-GHz Radio in 65-nm CMOS," Manuscript to be summited to IEEE journal.

[68] J. Borremans, G. Mandal, V. Giannini, et al., "A $40 \mathrm{~nm}$ CMOS 0.4-6 GHz Receiver Resilient to Out-of-Band Blockers," IEEE Journal of Solid-State Circuits, vol.46, no.7, pp.1659-1671, July 2011.

[69] Fahad Qazi, Quoc-Tai Duong, and Jerzy J. Dabrowski, "Tunable Selective Receiver Front-End with Impedance Transformation Filtering," Journal of Circuit Theory and Applications, August 2015, DOI: 10.1002/cta.2125.

[70] M. Mehrpoo and R. B. Staszewski, "A Highly Selective LNTA Capable of LargeSignal Handling for RF Receiver Front-Ends," in Proc. IEEE RFIC Symp., Jun. 2013, pp. 185-188, 2013.

[71] L. Zhang, et al., "Analysis and Design of a $0.6-10.5 \mathrm{GHz}$ LNTA for Wideband Receivers," Trans. On Circuits and Systems II, Express Briefs, vol. 62, no. 5, pp. 431-435, May 2015.

[72] A. Mirzaei, et al., "A low-power process-scalable super-heterodyne receiver with integrated high-Q filters," J. of Solid-State Circuits, vol. 46, no 12, pp. 2920 2932, 2011.

[73] M. Darvishi, et al., "Design of Active N-Path Filters," J. of Solid-State Circuits, vol. 48, no 12, pp. 2962-2976, 2013.

[74] J. Y. Ryu and B. C. Kim, "Low-cost testing of $5 \mathrm{GHz}$ low noise amplifiers using new RF BIST circuit," JETTA, vol.21, no.6, 2005.

[75] D. Han, and A. Chatterjee; "Robust Built-In Test of RF ICs Using Envelope Detectors" Proc. 14th Asian Test Symposium, pp. 2-7, 2005.

[76] R. Ramzan and J. Dąbrowski, "CMOS RF/DC Voltage Detector for on-chip Test," IEEE INMIC, pp. 472-476, 2006.

[77] C. Zhang, et al. "Low Cost RF Receiver Parameter Measurement with On-Chip Amplitude Detectors"in Proc. VTS , pp. 203-208, 2008.

[78] R. Ramzan and J. Dąbrowski. "On-chip Calibration of RF Detectors by DC Stimuli and ANN," RFIC Symp, pp. 571-574, 2008. 
[79] R. Musa, et al. "Design of Single-Stage Folded-Cascode Gain Boost Amplifier for $100 \mathrm{mV}$ 10-bit 50MS/s Pipelined Analog-to-Digital Converter," ICSE, pp. 800-804, 2006.

[80] Q-T. Duong and J. Dąbrowski, "Wideband RF Detector Design for High Performance On-Chip Test," IEEE NORCHIP Conf., 4 pp, 2012.

[81] S. B. Sleiman and M. Ismail, "Built-in-Self-Test and Digital Self-Calibration for RF SoCs," Springer, 2012.

[82] X. Fan, et al. "A Current Injection Built-In Test Technique for RF Low-Noise Amplifiers”, IEEE TCAS I, Vol.55, Aug. 2008, pp.1794-1804.

[83] S. Ahmad, "Stimuli Generation Techniques for On-Chip Mixed-Signal Test," PhD Th., ISBN: 978-91-7393-288-2, Linkoping, December 2010.

[84] R. K. Setty, "Digital step attenuators offer precision and linearity," in RF Design, pp. 34-42, Aug. 2005.

[85] D. Zhang, A. Alvandpour. "A 3-nW 9.1-ENOB SAR ADC at $0.7 \mathrm{~V}$ and $1 \mathrm{kS} / \mathrm{s}$," ESSCIRC, pp. 369-372, 2012.

[86] V. D. Bosch, et al.,"A 10-bit 1-GSample/s Nyquist current-steering CMOS D/A converter," IEEE J. Solid-State Circuits, vol. 36, pp. 315-324, Mar. 2001.

[87] S. Spiridon, et al., “A $375 \mathrm{~mW}$ Multimode DAC-Based Transmitter With $2.2 \mathrm{GHz}$ Signal Bandwidth and In-band IM3 < -58 dBc in 40nm CMOS," IEEE J. S.S. Circuits, vol. 48, pp. 1595-1604, July. 2013.

[88] D. A. Mercer,"Low Power Approaches to High Speed CMOS Current-steering DACs,” CICC, pp. 153-160, Sept. 2006.

[89] J. L. Dawson, "Feedback Linearization of RF Power Amplifiers," PhD Thesis, Stanford, US, August 2003.

[90] A. Afsahi, et al., "Linearized Dual-Band Power Amplifer with Integrated Baluns in 65nm CMOS for a 2x2 802.11n MIMO WLAN SoC," IEEE J. Solid-State Circuits, vol. 45, pp. 995-966, May. 2010.

[91] H. Hedayati, et al.,"A 2-GHz Highly Linear Efficient Dual-Mode BiCMOS Power Amplifier Using a Reconfigurable Matching Network," IEEE J. SolidState Circuits, vol. 47, pp. 2385-2404, Oct. 2012. 
[92] Q. Liu, et al.,"A high efficiency and high linearity power amplifier utilizing postlinearization technique for 5.8GHz DSRC applications," PAWR, pp. 45-48, Jan. 2011.

[93] C. Xin, et al., "A linearization technique for RF low noise amplifier," ISCAS, pp. 313-316, May. 2004.

[94] Y. Ding, et al.,"A Highly-efficiency CMOS +22-dBm Linear Power Amplifier," IEEE J. Solid-State Circuits, vol. 39, pp. 223-229, Jan. 2004.

[95] A. I. Galal, et al., "Comparision between bipolar and NMOS transistors in linearization technique at 5GHz low noise amplifier," APMC, pp. 1-4, Dec. 2008.

[96] F. J. Wang, et al., "A quasipassive CMOS pipeline D/A converter," IEEE J. SolidState Circuits, vol. 24, pp. 1752-1755, Dec. 1989.

[97] R. E. Suarez, et al.,"All-MOS Charge Redistribution Analog-to-Digital Conversion Techniques-Part II," IEEE J. Solid-State Circuits, vol. 10, pp. 379385, Dec. 1975.

[98] R. Gregorian, G. Temes, “Analog MOS Integrated Circuits”, Wiley, 1986

[99] T. Wakimoto, et al.,"Statistical Analysis on the Effect of Capacitance Mismatch in a High-resolution Successive-Approximation ADC," IEEJ Transactions on electrical and electronic engineering, vol. 6, pp. s89-s93, 2001.

[100] Q-T. Duong, J. Dąbrowski, A. Alvandpour, "Highly linear open loop output driver for high speed capacitive DACs", Norchip2013, pp. 1-4, 2013

[101] B. H. Leung, VLSI for Wireless Communication. Englewood Cliffs, NJ: PrenticeHall, 2002.

[102] R. van de Plassche, CMOS Integrated Analog-to-Digital and Digital-to-Analog Converters. $2^{\text {nd }}$ Edition, Springer, 2007.

[103] P. Palmers, et al.,"A 10-Bit 1.6-GS/s 27-mW Current-Steering D/A Converter With 550-MHz 54-dB SFDR Bandwidth in 130-nm CMOS," IEEE TCASI, vol. 57, pp. 2870-2879, Nov. 2010.

[104] M. G. Yuriy, et al.,"A 56GS/s 6b DAC in 65nm CMOS with 256x6b Memory," ISSC, vol. 10, pp. 194-195, Dec. 2011. 
[105] S. Jafar, et al., “A 12-GS/s Phase-Calibrated CMOS Digital-to-Analog for Backplane Communication," IEEE J. Solid-State Circuits, vol. 43, pp. 12071216, May. 2008.

[106] A. Bhide, et al., “ An 8-GS/s 200-MHz Bandwidth 68-mW $\Delta \Sigma$ DAC in 65-nm CMOS,” IEEE TCAS II, vol. 60, pp. 387-391, July. 2013.

[107] B. Kim, et al.,"A new linearization technique for MOSFET RF amplifier using multiple gated transistors," IEEE Microwave and guided wave letter, vol. 10, no. 9, pp. 270-273, Jan. 2000.

[108] Bhide A, et al., "An 11-GS/s 1.1-GHz bandwidth Interleaved $\Delta \sum \mathrm{DAC}$ for $60-\mathrm{GHz}$ Radio in 65-nm CMOS," IEEE J. Solid-State Circuits, vol. 50, pp. 2306-2318, Oct. 2015.

[109] R. van de Plassche, CMOS Integrated Analog-to-Digital and Digital-to-Analog Converters. $2^{\text {nd }}$ Edition, Springer, 2007.

[110] Li Ding and Pinaki. M,"Simultaneous switching noise analysis using application specific device modeling," IEEE Trans. VLSI, vol. 11, pp. 1146-1152, Dec. 2003.

[111] Yungseon Eo, et al.,"New simultaneous switching noise analysis and modeling for high-speed and high-density CMOS IC package design," IEEE Trans. Advanced packaging, vol. 23, pp. 303-312, May. 2000.

[112] Srinivasa R. V,“Accurate simultaneous switching noise estimation including velocity-saturation effects," IEEE Trans. Components, packaging and manufacturing technology, vol. 19, pp. 344-349, May. 1996.

[113] T. Lee, The Design of COS Radio-Frequency Integrated Circuits. $2^{\text {nd }}$ Edition, Cambridge University Press, 2004.

[114] Q. Gu, RF system design of transceivers for wireless communications. New York, 2005.

[115] Quoc-Tai Duong, et al.,“ Analysis and Design of Low Noise Transconductance Amplifier for Selective Receiver Frontend," Journal of Analog Integrated Circuits and Signal Processing, vol. 85, no. 2, pp. 361-372, Nov. 2015. 


\section{Paper Collections}

\section{Journals}

- J 1 - Quoc-Tai Duong, Fahad Qazi, and Jerzy J. Dabrowski, “Analysis and Design of Low Noise Transconductance Amplifier for Selective Receiver Frontend," Journal of Analog Integrated Circuits and Signal Processing, vol. 85, no. 2, pp. 361-372, Nov. 2015.

- J 2 - Fahad Qazi, Quoc-Tai Duong, and Jerzy J. Dabrowski, "Two-Stage Highly Selective Receiver Front-End Based on Impedance Transformation Filtering," IEEE Transactions on Circuits and Systems II: Express Briefs (TCAS-II), vol. 62, no. 5, pp. 421-425, May. 2015.

- J 3 - Fahad Qazi, Quoc-Tai Duong, and Jerzy J. Dabrowski, "Tunable Selective Receiver Front-End with Impedance Transformation Filtering," Journal of Circuit Theory and Applications, August 2015, DOI: 10.1002/cta.2125. 
- J 4 - Quoc-Tai Duong, Jerzy J. Dabrowski, and Atila Alvandpour, "Design and analysis of high speed capacitive pipeline DACs," Journal of Analog Integrated Circuits and Signal Processing, vol. 80, no. 3, pp. 359-374, Sept. 2014.

The following journal paper based on Chapter 6 is not added to the paper collections since it is submitted and not published yet.

- J 5 - Quoc-Tai Duong, Ameya Bhide, and Atila Alvandpour, "A 1-GHz Bandwidth 12-bit SC DAC for 60-GHz Radio in 65-nm CMOS," Manuscript to be summited to IEEE journal.

\section{Conferences}

- C 1 - Quoc-Tai Duong, and Jerzy J. Dabrowski, "Low Noise Transconductance Amplifier Design for Continuous-Time delta sigma Wideband Frontend," IEEE European Conference on Circuit Theory and Design (ECCTD), Linkoping, Sweden, pp. 825-828, August 2011.

- C 2 - Quoc-Tai Duong, and Jerzy J. Dabrowski, "Wideband RF Detector Design for High Performance On-Chip Test," IEEE Norchip, Copenhagen, Danmark, pp. 1-4, Nov 2012.

- C 3 - Quoc-Tai Duong, and Jerzy J. Dabrowski, "Focused calibration for advanced RF test with embedded RF detectors," IEEE European Conference on Circuit Theory and Design (ECCTD), Dresden, Germany, pp. 1-4, September 2013.

- $\quad$ C 4 - Quoc-Tai Duong, Jerzy J. Dabrowski, and Atila Alvandpour, "Highly linear open-loop output driver design for high speed capacitive DACs," IEEE Norchip, Vilnius, Lithuania, pp. 1-4, Nov 2013. 


\section{Papers}

The articles associated with this thesis have been removed for copyright reasons. For more details about these see:

http://urn.kb.se/resolve?urn=urn:nbn:se:liu:diva-124006 